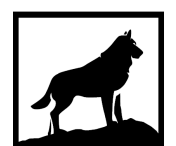

Michigan

Technological

1 8 8 5 University
Michigan Technological University

Digital Commons @ Michigan Tech

2015

ANTHROPOMORPHIC ROBOTIC ANKLE-FOOT PROSTHESIS WITH ACTIVE DORSIFLEXION- PLANTARFLEXION AND INVERSIONEVERSION

Evandro Ficanha

Michigan Technological University, emficanh@mtu.edu

Copyright 2015 Evandro Ficanha

Recommended Citation

Ficanha, Evandro, "ANTHROPOMORPHIC ROBOTIC ANKLE-FOOT PROSTHESIS WITH ACTIVE DORSIFLEXION- PLANTARFLEXION AND INVERSION-EVERSION", Open Access Dissertation, Michigan Technological University, 2015.

https://doi.org/10.37099/mtu.dc.etdr/15

Follow this and additional works at: https://digitalcommons.mtu.edu/etdr

Part of the Biomechanical Engineering Commons 


\title{
ANTHROPOMORPHIC ROBOTIC ANKLE-FOOT PROSTHESIS WITH ACTIVE DORSIFLEXION- PLANTARFLEXION AND INVERSION-EVERSION
}

By

Evandro M. Ficanha

\begin{abstract}
A DISSERTATION
Submitted in partial fulfillment of the requirements for the degree of DOCTOR OF PHILOSOPHY

In Mechanical Engineering-Engineering Mechanics
\end{abstract}

MICHIGAN TECHNOLOGICAL UNIVERSITY

2015

(C) 2015 Evandro M. Ficanha 
This dissertation has been approved in partial fulfillment of the requirements for the Degree of DOCTOR OF PHILOSOPHY in Mechanical Engineering-Engineering Mechanics.

Department of Mechanical Engineering-Engineering Mechanics

Dissertation Advisor: $\quad$ Mohammad Rastgaar

Committee Member: Jason R. Blough

Committee Member: Kenton Kaufman

Committee Member: Nina Mahmoudian

Committee Member: Gordon G. Parker

Department Chair: William Predebon 
In memory of Jack Harris. For giving me the chance to follow my dreams. 


\section{TABLE OF CONTENTS}

1- Preface

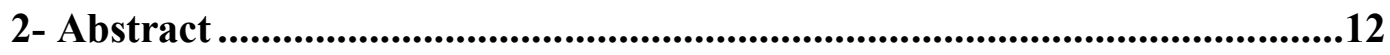

3- Introduction.............................................................................................13

4- Ankle Kinematics Describing Gait Agility: Considerations in the Design of an Agile Ankle-Foot Prosthesis ........................................................................18

4.1 Abstract ..................................................................... 18

4.2 Introduction ................................................................... 18

4.3 Ankle Rotations during Gait............................................21

4.3.1 Ankle Rotations during the Stance Period of Step Turn ......22

4.3.2 Ankle Rotations Step Turn at Different Gait Speeds ..........25

4.4 Conclusion ....................................................................26

5-Ankle Mechanics during Sidestep Cutting Implicates Need for 2-DOF Powered Ankle-foot Prostheses..........................................................................28

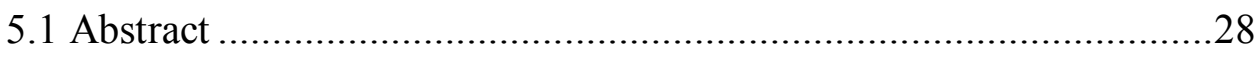

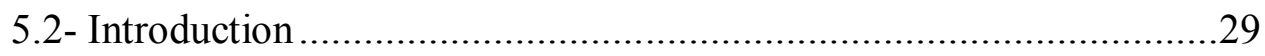

5.3- Ankle Kinematics and Kinetics during Gait ................................32

5.4- Experiment Setup and Analysis ............................................32

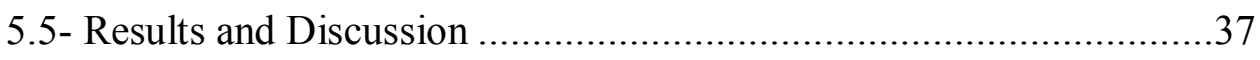

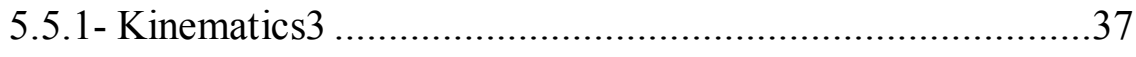

5.5.1.1- Sagittal Plane Kinematics ............................. 37 
5.5.1.2 Frontal plane kinematics ...............................39

5.5.1.3 Transverse plane kinematics............................40

5.5.2- Kinetics ................................................................4 42

5.5.2.1 Sagittal Plane Kinetics ..................................42

5.5.2.2 Frontal Plane Kinetics ...................................44

5.5.2.3 Transverse Plane Kinetics ................................45

5.6 Remarks on the Ankle Impedance ............................................47

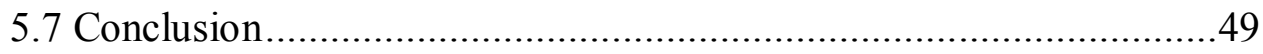

6- Multi-axis Capability for Powered Ankle-foot Prostheses ..........................50

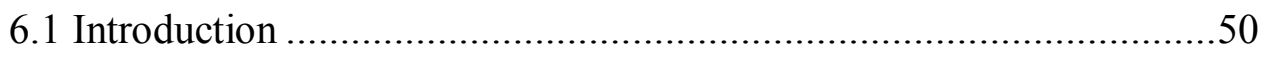

6.2- Multi-axis Ankle-foot Prototype ..............................................54

6.3 Evaluation of the Design Concept ...............................................59

6.3.1 Kinematic Evaluation .............................................45

6.3.2 Mechanical Impedance Estimation .............................60

7- Gait Emulator for Evaluation of Ankle-Foot Prostheses Capable of Turning ....................................................................................................................65

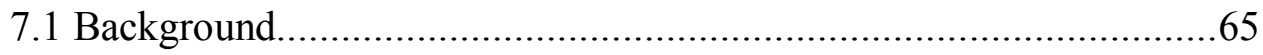

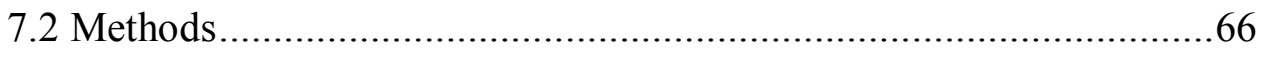

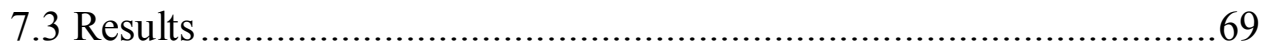

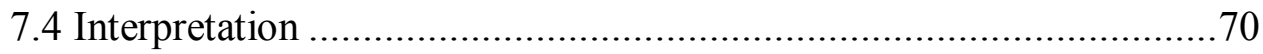

8- Impedance and Admittance Controller for a Multi-axis Powered Anklefoot Prosthesis..............................................................................................71 


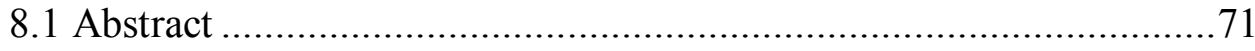

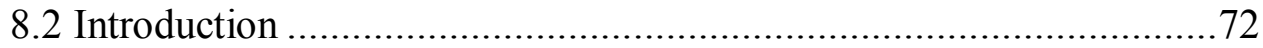

8.3 Cable-driven Powered Ankle-foot Prosthesis with Two Controllable

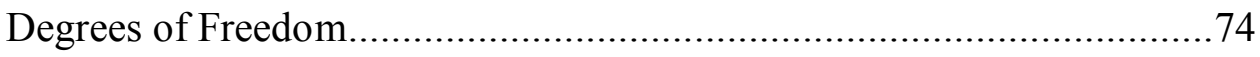

8.4 Ankle Torque and Angle Feedback ............................................. 76

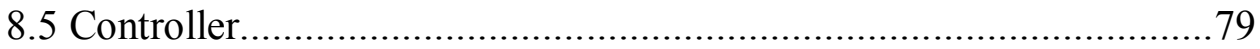

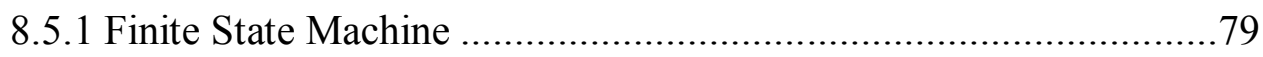

8.5.2 Impedance Controller ................................................81

8.5.3 Admittance Controller ...................................................82

8.6 Preliminary Evaluation Experiments ....................................... 84

8.6.1 Impedance Controller in Quasi-static Condition.................84

8.6.2 Prosthesis Test on the Circular Treadmill........................88

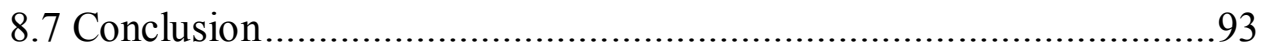

\section{9- Design and Evaluation of a 2-Dof Instrumented Platform for Estimation of the Ankle Mechanical Impedance during Gait in Arbitrary Directions ........94}

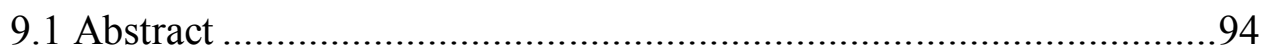

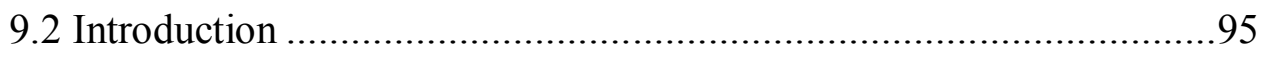

9.3 Instrumented Vibrating Platform Design ....................................98

9.3.1 Actuation Module Design.........................................99

9.3.2 Force Plate Module...................................................... 102

9.3.3 Instrumented Walkway ......................................... 111

9.4 System Validation Using a Mockup ....................................... 112

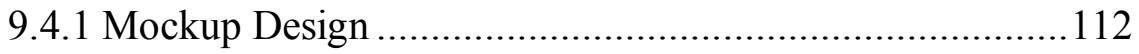


9.4.2 Ankle Center of Rotation Calculation ........................... 113

9.4.3 Quasi-Static Stiffness Measurement...............................116

9.4.4 Mockup Impedance Measurement Using a Single-Variable Stochastic Identification Method ......................................... 117

9.4.5 Mockup Impedance Measurement Using a Multi-Variable Stochastic Identification Method .............................................119

9.5 Preliminary Evaluation of the Time-Varying Mechanical Impedance of the Human Ankle .............................................................. 120

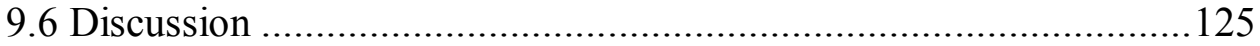

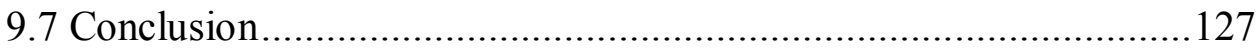

\section{0-Mechanical Impedance of the Non-Loaded Lower Leg with Relaxed Muscles in the Transverse Plane ............................................................129}

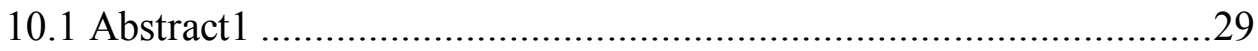

10.2 Introduction .................................................................. 130

10.3 Experiment Methodology ................................................. 134

10.3.1Human Subjects..................................................... 134

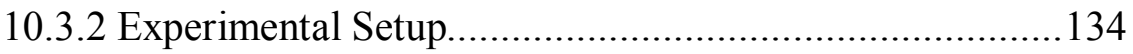

10.3.3 Dynamic Impedance Estimation .............................138

10.3.4 Quasi-static Stiffness Measurement ........................... 140

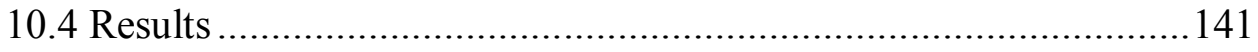

10.4.1 Results for Dynamic Impedance ................................ 141

10.4.2 Results for Quasi-static Stiffness ................................144

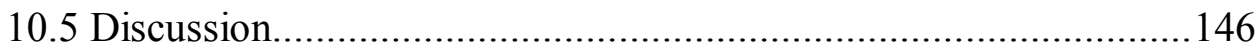


10.6 Conclusion.................................................................. 148

\section{1- Design of a Steerable Powered Ankle-Foot Prosthesis Capable of Turning ........................................................................................150}

11.1 Abstract ................................................................... 150

11.2 Introduction .................................................................. 150

11.3 Design detail of the 2-DOF ankle foot prosthesis........................154

11.4 Human ankle kinematics tracking ........................................157

11.5 Ankle torque feedback for impedance controllers .......................158

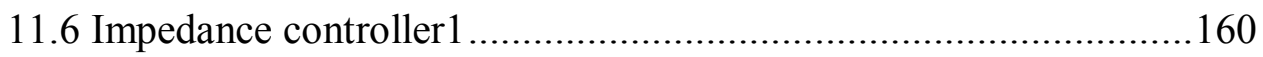

11.6.1 Impedance controller development ............................... 160

11.6.2 Experimental estimation of the ankle-foot prosthesis quasistatic impedance and impedance gain calibration......................162

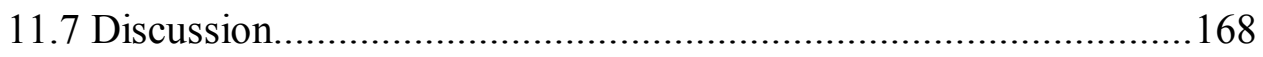

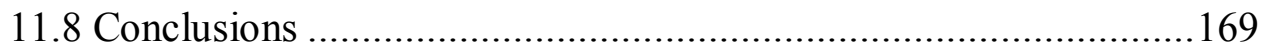

12- Concluding Remarks.....................................................................171

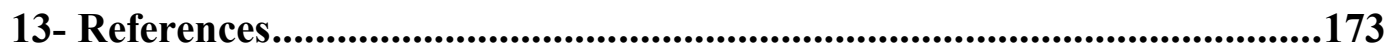

14- Appendices....................................................................................187

14.1 Appendix A - IEEE copyright agreement ..............................187

14.2 Appendix B - JRDD copyright agreement ............................. 189

14.3 Appendix C - Springer copyright agreement............................190

14.4 Appendix D - ASME copyright agreement .............................193 


\section{1- PREFACE}

This dissertation is submitted in partial fulfillment of the requirements for a Degree of Doctor of Philosophy $(\mathrm{PhD})$ in Mechanical-Engineering. The work was conducted at the Mechanical Engineering-Engineering Mechanics PhD Program at Michigan Technological University under the supervision of Dr. Mohammad Rastgaar.

The research presented in this dissertation has been done by the author as well as in collaboration with others: Dr. Mohammad Rastgaar, Dr. Kenton R. Kaufman, Mr. Ruiyu Kang, and Mr. Guilherme Ribeiro.

Chapter 4 - Ankle Kinematics Describing Gait Agility: Considerations in the Design of an Agile Ankle-Foot Prosthesis - was written in collaboration with $\mathrm{Mr}$. Evandro Ficanha (data acquisition, and data analysis), Ruiyu Kang (data acquisition), and Dr. Mohammad Rastgaar (data analysis). The material contained in this chapter is part of material previously published in the 2014 IEEE International Conference on Biomedical Robotics and Biomechatronics. Permission to use the material is shown in appendix $\mathrm{A}$.

Chapter 5 - Ankle Mechanics during Sidestep Cutting Implicates Need for 2DOF Powered Ankle-foot Prostheses - was written in collaboration with Mr. Evandro Ficanha (data acquisition, and data analysis), Dr. Rastgaar (data analysis) and Dr. Kenton R. Kaufman (data analysis). The material contained in this chapter was previously published in the Journal of Rehabilitation Research \& Development. Permission to use the material is shown in appendix B.

Chapter 6 - Multi-axis Capability for Powered Ankle-foot Prostheses - was written in collaboration with Mr. Evandro Ficanha (Hardware development, tests, and data analysis), Dr. Rastgaar (data analysis) and Dr. Kenton R. Kaufman (data analysis). The material contained in this chapter is part of material previously 
published in the book: Neuro-Robotics: From Brain Machine Interfaces to Rehabilitation Robotics. Permission to use the material is shown in appendix C.

Chapter 7 - Gait Emulator for Evaluation of Ankle-Foot Prostheses Capable of Turning - was written in collaboration with Mr. Evandro Ficanha (Hardware development, tests, and data analysis), Dr. Rastgaar (data analysis) and Dr. Kenton R. Kaufman (data analysis). The material contained in this chapter was previously published in the ASME Journal of Medical Devices. Permission to use the material is shown in appendix D.

Chapter 8 - Impedance and Admittance Controller for a Multi-Axis Powered Ankle-Foot Prosthesis - was written in collaboration with Mr. Evandro Ficanha (Hardware development, tests, and data analysis) and Dr. Mohammad Rastgaar (data analysis). The material contained in this chapter is part of material previously published in the 2014 ASME Dynamic Systems and Control Conference. Permission to use the material is shown in appendix D.

Chapter 9 - Design and Evaluation Of a 2-DOF Instrumented Platform for Estimation of the Ankle Mechanical Impedance during Gait in Arbitrary Directionswas written in collaboration with Mr. Evandro Ficanha (Hardware development, tests, and data analysis), Mr. Guilherme Ribeiro (data analysis) and Dr. Mohammad Rastgaar (data analysis). The material contained in this chapter is in preparation for submission to a journal.

Chapter 10 - Mechanical Impedance of the human ankle in External-Internal Direction - was written in collaboration with. Mr. Evandro Ficanha (hardware, experiments, and data analysis), Mr. Guilherme Ribeiro (data experiments and analysis) and Dr. Mohammad Rastgaar (data analysis). The material contained in this chapter is in preparation for submission to a journal.

Chapter 11 - Design of a Robotic Ankle-Foot Prosthesis with Active Dorsiflexion-Plantarflexion and Inversion-Eversion - was written in collaboration with. Mr. Evandro Ficanha (hardware, experiments, and data analysis), Mr. 
Guilherme Ribeiro (data analysis) and Dr. Mohammad Rastgaar (data analysis). The material contained in this chapter is in preparation for submission to a journal. 


\section{2- ABSTRACT}

The main goal of the research presented in this paper is the development of a powered ankle-foot prosthesis with anthropomorphic characteristics to facilitate turning, walking on irregular grounds, and reducing secondary injuries on bellow knee amputees. The research includes the study of the gait in unimpaired human subjects that includes the kinetics and kinematics of the ankle during different types of gait, in different gait speeds at different turning maneuvers. The development of a robotic ankle-foot prosthesis with two active degrees of freedom (DOF) controlled using admittance and impedance controllers is presented. Also, a novel testing apparatus for estimation of the ankle mechanical impedance in two DOF is presented. The testing apparatus allows the estimation of the time-varying impedance of the human ankle in stance phase during walking in arbitrary directions. The presented work gives insight on the turning mechanisms of the human ankle and how they can be mimicked by the prosthesis to improve the gait and agility of below-knee amputees. 


\section{3- INTRODUCTION}

The ankle is the first major joint that transfers the ground reaction torques to the rest of the body. Activities of daily leaving (ADLs) involve different tasks that may require the ankle function in all anatomical planes. These activities include, but are not limited to, standing, normal walking, walking on incline planes, turning around corners, avoiding collisions, and climbing/descending stairs. Turning steps may represent $8 \%$ to $50 \%$ of all steps depending on the activity (1). Due to the significant role of the ankle in ADLs, the development of ankle-foot prostheses should take the mechanical characteristics of the human ankle into consideration. Currently there are nearly 2 million amputees in the United States (2). The main causes of amputations are vascular diseases (54\%), which includes diabetes and peripheral arterial disease, and trauma (45\%) (2); this mortality rate of amputees due to vascular diseases is $50 \%$ within the first 5 years. This 5 year mortality rate is higher than breast cancer, colon cancer, and prostate cancer (3). Lower limb accounts for $97 \%$ of all amputations due to vascular diseases (4), resulting in nearly 1 million lower limb amputees in the United States. Ankle-foot prostheses with anthropomorphic characteristics may decrease the metabolic cost while generating a more comfortable gait and decreasing secondary injuries due to overuse or misuse of other joints. These may lead to an increase in mobility and activity levels, a reduction on the likelihood of obesity and cardiovascular diseases, and an overall improvement of the quality of life in amputees.

The aim of the research presented in this dissertation was to create an ankle-foot prosthesis with two DOF in the sagittal and frontal planes. The ankle rotations in the sagittal plane are Dorsiflexion and Plantarflexion (DP), which are the movements of the foot downwards and upwards, respectively. The ankle rotations in the frontal plane are Inversion and Eversion (IE), which are the roll of the foot inwards or outwards, respectively. 
Currently, available powered ankle-foot prostheses focus on improving mobility by powering the ankle joint in the sagittal plane; however, there is substantial ankle function in all anatomical planes, even during straight walk on level ground (5). Much research has focused on straight walk and the dorsiflexion-plantarflexion of the ankle, while less attention has been given to the turning mechanism, although it plays a major role in locomotion (6). As an example, amputees use their hip mainly in the sagittal plane to turn in such a way that the outside step length is longer than the inside step length, causing the body to rotate without the need to lean the body (7). Non-amputees rely mainly on the ankle rotations in the sagittal plane and hip rotations in the frontal plane to tilt the body towards the inside of the turn (7). It is estimated that the different strategies are required to compensate for the lack of propulsion in passive prostheses to increase stability and maneuverability (7). This evidence suggests that an ankle-foot prosthesis controllable in both DP and IE directions may provide more assistance in conforming the foot to the ground profile and uneven surfaces, walking in arbitrary directions on the slopes, steering, and turning.

Currently, there are few commercially available transtibial powered prostheses that actively control one DOF in the sagittal plane $(8-10) \cdot$ BiOM $^{\circledR}$ provides the necessary energy during toe-off (plantarflexion); therefore, it actively contributes in gait and lowers the metabolic cost. The controller in $\mathrm{BiOM}^{\circledR}$ allows for gait in different cadences over surfaces with different inclination in uphill and downhill trajectories (8). Proprio Foot $^{\circledR}$ from Össur uses a stepper motor to provide dorsiflexion motion during swing forward and adjustment of the ankle angle on the surface with different trains. The controller uses a pattern recognition algorithm to adapt to the human's gait continuously (9). Élan from Endolite uses a hydraulic ankle and the controller provides both dorsiflexion for foot clearance and plantarflexion for support during stance by adjusting the ankle joint resistance (10). In the transverse plane, Olson et.al developed a transtibial prosthesis with active transverse plane control to reduce the shear stress and the rotation of the residual 
limb in the socket(11). The rotation of the residual limb in the socket can lead to abrasion and skin problems (12). To the author's best knowledge, there is currently no powered-ankle foot prosthesis with control in both the sagittal and frontal planes.

The human ankle is composed of the talocrural joint and the subtalar joint. These joints are not aligned with the anatomical axes, and the rotation of the ankle about each of these joints results in combined rotations in the anatomical reference frame. The talocrural joint combines dorsiflexion with lateral rotations and combines plantarflexion with medial rotations. The subtalar joint combines dorsiflexion, eversion, and external rotations and combines plantarflexion, inversion, and internal rotations (13). The complex structure of the human ankle makes it hard to mimic the same kinetics and kinematics behavior on prostheses. In the development of powered ankle-foot prostheses, it is generally acceptable not to power the ankle in the transverse plane since the hip joint is capable of generating torques and rotations in that plane; however, this torque can cause skin problems due to the shear stress and the rotations of the residual limb in the socket (11). To reduce the socket torque, compliant passive transverse rotation adaptors are commonly used in passive and active prostheses, and were reported to greatly reduce skin abrasions (12). The work presented focuses on the development of an ankle-foot prosthesis to improve mobility, thus it is focused on the ankle activation in the frontal and sagittal planes.

The ankle kinematics during a step turn (a $90^{\circ}$ turn similar to turning around a corner) was studied on healthy subjects using a motion capture camera system (discussed in chapter 4). The findings provided evidence for necessity of 2-DOF ankle-foot designs while providing the preliminary design parameters for a prototype 2-DOF powered ankle-foot prosthesis. Chapter 5 expands the work presented in chapter 4 by studding the ankle kinetics and kinematics during sidestep cutting (a step where the leading leg push the body sideways near or at $45^{\circ}$ to avoid an obstacle on the ground while walking forward) and straight walking at two 
different gait speeds, showing great variability on the ankle kinetics and kinematics in IE when comparing straight walk to turning.

Based on the human ankle kinetics and kinematics, a powered ankle-foot prosthesis with two DOF capable of turning was developed (discussed in chapter 6). The prototype was capable of properly mimicking the human ankle kinematics, and was able to generate enough torque to lift a $72 \mathrm{~kg}$ person. To test the prosthesis, a gait emulator for evaluation of ankle-foot prostheses capable of turning was developed (discussed in chapter 7). The gait emulator provided a platform for testing the ankle-foot prosthesis, allowing for consistent and repeatable measurements, and tuning the controllers of the powered ankle-foot prosthesis. Impedance and admittance controllers were developed for the prosthesis (discussed in chapter 8). Strain gauges were installed on the prosthesis' foot to measure the strain caused by ground reaction forces, which are correlated to the external torques in DP and IE. The external torques were used on admittance and impedance controllers. A preliminary finite state machine was used to select between impedance and admittance controllers for the ankle-foot prosthesis using the strain gauges feedback. In the beginning of the step cycle, when heel-strike was detected, the finite state machine switches to admittance control. The admittance control accepts torque feedback to generate motion, this way larger feedback torques effectively reduce the stiffness of the ankle. During push off, the finite state machine switches to impedance control, accepting motion feedback to generate the appropriated torques required for locomotion.

Based on the impedance and admittance controllers of the prosthesis, it was concluded that there is a need to obtain time-varying mechanical impedance of the human ankle during walk to be used in the prosthesis. An instrumented vibrating walkway designed for estimation of the human ankle's mechanical impedance in two DOF during gait in arbitrary directions was fabricated and evaluated (discussed in chapter 9). The platform was designed to obtain the time-varying mechanical impedance of the human ankle in DP and IE during different types of walk, such as 
straight walk and turning maneuvers. The results using an ankle mockup showed that the developed system was capable of identifying the effects of the inertia, damping, and stiffness of the mockup. Preliminary results of the time-varying mechanical impedance of the human ankle (using a human sample subject) using ensemble-based linear time-varying system identification methods show the capability of the system and the method through most of the stance phase, except at early stance phase. The vibrating platform, however, does not support testing the ankle in the Internal-external direction (EI). The Ankle impedance was estimated in EI (discussed in chapter 10), where the impedance was tested using a robot (Anklebot) capable of applying torque perturbations to the ankle in EI with no load bearing of the ankle. The ankle impedance estimation in EI has the objective to facilitate the design of passive and active prostheses with mechanical characteristics similar to the human ankle in that axis of rotation. Powered ankle-foot prostheses do not necessarily need to power the ankle in the transverse plane since the hip joint is capable of generating torques and rotations in the transverse plane; however, the ankle joint may benefit from a passive stiffness close to the human ankle in EI.

A second generation of the powered ankle-foot prosthesis was developed (discussed in chapter 11). It adds a Bowden cable drive system which allows the placement of the motors and gear boxes in arbitrary location, for example, in a backpack. Therefore, it can greatly improve the metabolic cost as the user is not required to accelerate the mass of these components at each step. Also, it allows for amputees with long residual limbs to benefit from a powered prosthesis that can easily be tailored to their specific sizes, as such individuals may not have enough space in their residual limb to accommodate motors and gear boxes. Additionally, by using higher end components, both the kinetics and kinematics of the second generation prosthesis were improved upon the previous model. 


\section{4- ANKLE KINEMATICS DESCRIBING GAIT AGILITY: CONSIDERATIONS IN THE DESIGN OF AN AGILE ANKLE-FOOT PROSTHESIS*}

Evandro M. Ficanha, Ruiyu Kang, and Mohammad Rastgaar

\subsection{ABSTRACT}

The designs of available lower extremity powered prostheses are focused on a single degree of freedom (DOF) in sagittal plane, allowing the control of their ankle joints in dorsiflexion and plantarflexion. The human gait however, shows that the ankle movements in both sagittal and frontal planes are significant even during walking on a straight path. Additionally, there is a significant change in the ankle movements during straight walking compared to turning steps, especially in the frontal plane. A better understanding of the ankle characteristics in both sagittal and frontal planes may result in the design of significantly more effective lower extremity prostheses that mimic the ankle function and improve the agility of gait.

In this paper, the ankle rotations are estimated during step turn to provide evidence for necessity of a multi-axis design while providing the preliminary design parameters for a prototype multi-axis powered ankle-foot prosthesis.

\subsection{INTRODUCTION}

Many gait scenarios such as traversing slopes or turning requires agile movements of the ankle in both sagittal and frontal planes. Agility is defined as the ability to move quickly and easily (14) and it is fundamental for a natural and efficient gait. Agility is essential when changing directions or accommodating disturbances on the terrain to minimize energy consumption and reduce the risk of injury.

*The material contained in this chapter is part of material previously published in the 2014 IEEE International Conference on Biomedical Robotics and Biomechatronics. The permission to use the material is shown in appendix A. 
Recent advances in powered prostheses promise to significantly improve the quality of life of individuals with impaired mobility. A better understanding of the complexities surrounding lower limb prostheses, will lead to increased health and well-being for the 1.7 million limb amputees in the US, the majority of whom have lower extremity amputations $(2,15)$. Currently commercially available powered ankle-foot prostheses are capable of controlling a single DOF in the sagittal plane, focusing on improved mobility in straight walking even though turning steps represents an average of $\sim 25 \%$ of steps taken during a typical day (1). Because current prostheses are not designed to assist turning, amputees and non-amputees exhibit different turning strategies. During turning non-amputees typically generate most propulsion at the ankle and the hip movement in the frontal plane. In contrast, amputees using a passive prosthesis generate propulsion by moving the hip in the sagittal plane. It is suggested that such difference in gait strategies are due to lack of sufficient power in the prosthetic ankle and the amputees' desire to prevent fall (6, $7,16,17)$. Such differences in gait strategies lead to a different biomechanics of turn and increased risk of secondary complications. During a turn, ground reaction forces are modulated to accelerate the center of mass of the body along the path; thus, during a step turn, lateral and propulsive impulses are larger compared to a straight step (18); also, preliminary studies have shown an increase in inversion during a step turn, leaning the body toward the inside of the turn, when compared to a straight step (19). These evidences suggest that turning may not be considered a passive mechanism and requires modulation of ankle impedance in both sagittal and frontal planes. Therefore, we theorize that an ankle-foot prosthetic robot capable of generating torques in both the dorsiflexion-plantarflexion (DP) and inversioneversion (IE) directions with impedance modulation similar to the human ankle may improve the user's agility and increase mobility while reducing the risk of secondary injuries or falls.

Understanding of the ankle's capability in impedance modulation and generating net positive work during the stance period of gait has influenced the design of new 
ankle-foot prostheses (20-22). One design approach is based on storing energy during the heel strike and releasing it during the push-off before the trailing foot's heel strike. Collins and Kuo $(23,24)$ developed a microprocessor-controlled artificial foot that limits the increase in metabolic cost to $14 \%$ compared to $23 \%$ that occurs with a passive prosthesis. On the other hand, there are powered prostheses capable of injecting energy to the system. Sup et al. developed a powered transfemoral prosthesis with active knee and ankle joints, each with one controllable DOF in the sagittal plane (25-28). The controller adjusts the impedance at a number of instants during gait by altering the neutral position of the ankle. Au et al. developed the ankle-foot prosthesis $\mathrm{BiOM}{ }^{\circledR}$ (29-31), which provides the necessary energy during push off and generates a net positive work $(32,33)$ that has been shown to reduce the metabolic costs by $8.9 \%$ to $12.1 \%$ at different gait speeds compared to a passive prosthesis and increased the preferred gait speed by $23 \%$ (34).

While the aforementioned prostheses have advanced the state-of-the-art, their designs are confined to the sagittal plane. Even level walking in a straight line requires the ankle to function in both the sagittal and frontal planes. Additionally, normal daily activity includes more gait scenarios which requires agile movements such as turning, traversing slopes, steering, and adapting to uneven terrain profiles. This suggests that the next advancement in lower extremity assistive devices is to extend their design and control to the frontal plane. To do that, we need a better understanding of the multi-variable mechanical impedance of the human ankle which requires knowledge about the time history of the ankle angles and torques during different gait scenarios. The ankle displacements needs to be studied since the mechanical impedance of the ankle is a dynamic operator that maps the timehistory of angular displacements onto the corresponding time-history of torques at the ankle joint. In this paper, the ankle angles during straight walk and different turning scenarios were measured. We use the term step turn to describe the maneuver used to change the walking direction by pivoting around the leading 
leg and rotating into a new direction (approximately perpendicular to the initial direction such as turning around a corner). In this paper we described the experiments for collecting the information on the ankle angles during straight walk and turning.

\subsection{ANKLE ROTATIONS DURING GAIT}

To change the direction in gait, one needs to perform different gait maneuvers such as step turn, spin turn, or sidestep cutting that have different kinematics. For example, compared to straight walking, step turns have considerably different velocity, length, width, and higher turning reaction forces $(6,15,17,18)$. Also the ankle moment in the inversion direction is significantly different from the straight steps and spin turn steps(35).

A set of experiments were performed to quantify the kinematic behavior of the ankle in the context of agility of gait. The experiments measured the ankle rotations during stance period of step turn and compared the results to the ankle rotation during straight walking. The study however, did not include any cognitive aspect of the agility, but focused on the kinematics of the gait due to change of direction and speed. Additionally, the ankle rotations were used to provide design parameters for the range of motion (ROM) of the prosthesis and to evaluate the kinematic design of the ankle-foot prosthesis in reproducing the same trajectories.

There have been different approaches to measure ankle rotations during gait that include using flexible electro-goniometer, electromagnetic tracking devices, and motion capturing cameras $(6,17,18,35)$. We used a motion capture camera system to track the three-dimensional rotations of the foot and tibia in stance periods. The motion capture camera system consisted of eight cameras in a square formation covering a volume of about 16 cubic meters and an area of 12 square meters. The cameras emitted infrared light and captured the reflected light from reflectors mounted on the participants with a rate of $250 \mathrm{~Hz}$. Reflective markers were attached to polycarbonate plastic rigid bodies. One rigid body was attached to the 
participant's shin resting against the tibia bone to record the shin rotations. Another rigid body was attached to the user's shoe above the metatarsal bones to record the foot rotations. The ankle rotations were calculated as the relative angles between the foot and shin.

Subjects with no self-reported neuromuscular and biomechanical disorders were recruited for the experiments. The subjects gave written consent to participate in the experiment that was approved by the Michigan Technological University Institutional Review Board. The details of the experiments and the results follow.

\subsubsection{Ankle Rotations during the Stance Period of Step Turn}

Straight walking requires a complex sequence of muscle activation to modulate the ground reaction forces to produce forward motion. Similarly, modulation of the reaction forces is required for turning the body (7). Two different strategies that are commonly used for turning are the spin turn and the step turn. The spin turn consists of turning the body around the leading leg (e.g. turning right with the right leg in front). The step turn consists of shifting the body weight to the leading leg and stepping onto the opposite leg while still shifting the body weight (e.g. turning left with the right leg in front). It has been shown that the step turn velocity, length, and width are considerably different than the straight walk with higher turning reaction forces (18). Three-dimensional measurement of the ankle angles during step and spin turns have been previously studied (35); however, it is of interest to study the ankle angular displacements during different phases of the stance period of turning steps and compare these results to the ankle angles during straight steps.

Five male subjects participated in this study. The subjects were instructed to walk at a normal pace with an audible metronome synchronized to their number of steps per minute in an attempt to keep the walking speed constant. The gait speed for the participants ranged from 88 to 96 steps per minute. They started walking from outside the field of view of the cameras while following a straight line marked on the floor (Fig 4.1). When they reached a reference point on the floor, they 
performed a $90^{\circ}$ step turn to the left, pivoting on their right leg and continued walking straight until they were outside the field of view of the cameras. Each subject repeated the test nine times, after several training trials to increase the consistency of the trials. Time trajectories of the markers on the tibia and foot were used to estimate the ankle rotations in DP, IE and External-Internal (EI) directions. The data for each test was divided into 6 phases: Heel strike (consists of heel strike and loading response), mid stance, and push off (consisted of terminal stance and pre-swing phases) for both straight and turning steps. The averages of the DP, IE, and EI rotations of each phase were calculated for all 9 trials of 5 subjects (a total of 45 trials).

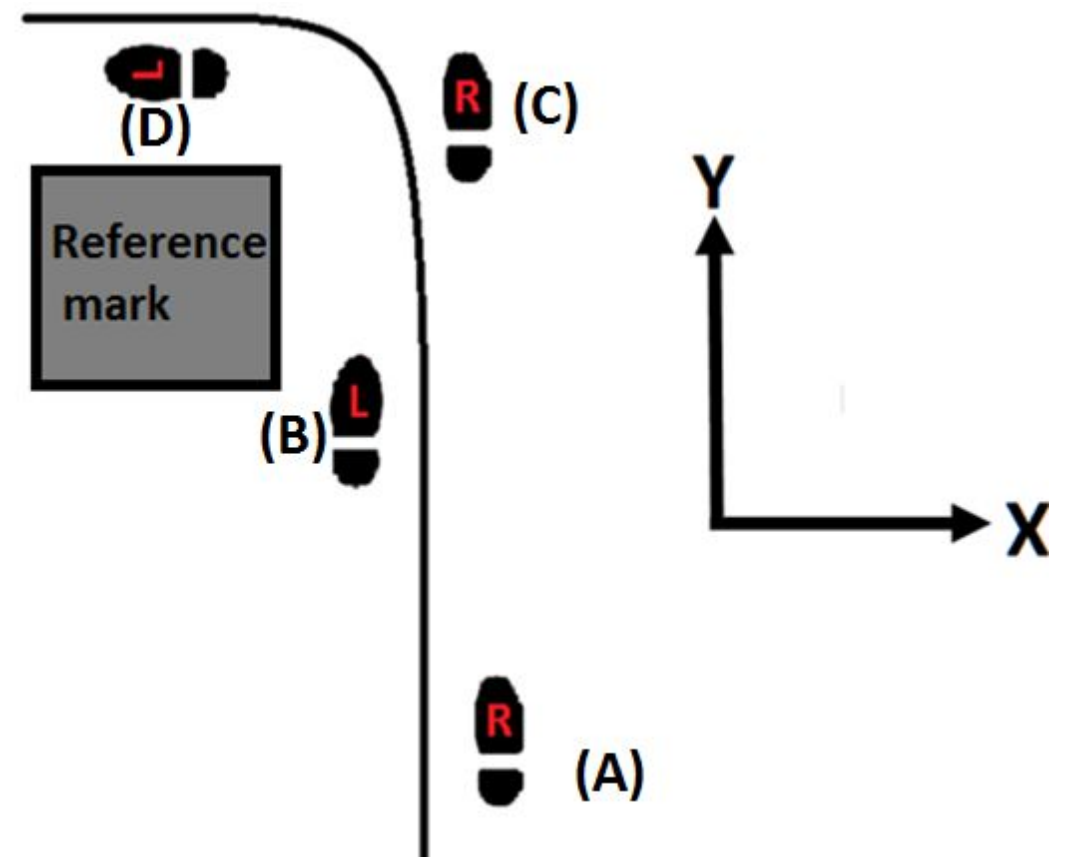

Figure 4.1. Foot positions during step turn test. (A) straight step right ankle. (B) straight step left ankle. (C) turn step right ankle. (D) post-turn left ankle.

Table 4.1 shows the average ROM of the subjects during the stance periods of straight step and step turn. Table 4.2 shows the average rotations and the difference in angles from the turning step to the straight step in each phase. The ROM of each subject's ankle about the three axes of the ankle and their average rotations during 
the stance periods were calculated and used to find the average percent change from straight walk to step turn with respect to their ROM during the straight step.

Table 4.1. Ankle ROM (in degrees) throughout stance for straight walk and step turn

\begin{tabular}{|c|c|c|c|c|}
\hline & $\begin{array}{c}\text { Straight Step } \\
\text { mean (std. } \\
\text { error) }\end{array}$ & $\begin{array}{c}\text { Step turn } \\
\text { mean (std. } \\
\text { error) }\end{array}$ & $\begin{array}{c}\text { Angular } \\
\text { Change }\end{array}$ & $\begin{array}{c}\text { Percent } \\
\text { Change }\end{array}$ \\
\hline DP & $33.9(0.7)$ & $31.6(0.6)$ & -2.3 & -7.4 \\
\hline IE & $15.7(0.5)$ & $20.6(1.1)$ & 4.9 & 23.8 \\
\hline EI & $22.1(0.6)$ & $16.8(0.7)$ & -5.3 & -31.9 \\
\hline
\end{tabular}

Table 4.2. Average ankle rotations (in degrees) during stance phases of straight

\begin{tabular}{|c|c|c|c|c|c|}
\hline \multicolumn{6}{|c|}{ steps and step turns } \\
\hline & & $\begin{array}{l}\text { Straight Step } \\
\text { Mean } \\
\text { (std. error) }\end{array}$ & $\begin{array}{l}\text { Step turn } \\
\text { Mean } \\
\text { (std. error) }\end{array}$ & $\begin{array}{l}\text { Angular } \\
\text { Change }\end{array}$ & $\begin{array}{c}\text { Percent } \\
\text { Change as a } \\
\text { Percent of } \\
\text { ROM }^{*} \\
\end{array}$ \\
\hline \multirow{3}{*}{ DP } & $\begin{array}{c}\text { heel } \\
\text { strike }\end{array}$ & $-8.7(0.8)$ & $-9.7(1.0)$ & -1.0 & -2.8 \\
\hline & $\begin{array}{c}\text { mid } \\
\text { stance }\end{array}$ & $2.3(0.6)$ & $0.4(0.6)$ & -2.0 & -5.8 \\
\hline & $\begin{array}{c}\text { push } \\
\text { off }\end{array}$ & $10.6(1.2)$ & $1.4(0.9)$ & -9.2 & -27.2 \\
\hline \multirow{3}{*}{ IE } & $\begin{array}{c}\text { heel } \\
\text { strike }\end{array}$ & $-1.7(0.5)$ & $5.9(0.6)$ & 7.6 & 48.5 \\
\hline & $\begin{array}{c}\text { mid } \\
\text { stance }\end{array}$ & $-2.9(0.3)$ & $6.5(0.2)$ & 9.4 & 60.1 \\
\hline & $\begin{array}{c}\text { Push } \\
\text { off }\end{array}$ & $1.4(0.5)$ & $13.6(0.5)$ & 12.2 & 77.5 \\
\hline \multirow{3}{*}{ EI } & $\begin{array}{c}\text { heel } \\
\text { strike }\end{array}$ & $-5.3(0.6)$ & $0.3(0.6)$ & 5.7 & 25.7 \\
\hline & $\begin{array}{c}\text { mid } \\
\text { stance }\end{array}$ & $-0.9(0.5)$ & $-3.6(0.4)$ & -2.7 & -12.0 \\
\hline & $\begin{array}{c}\text { push } \\
\text { off }\end{array}$ & $5.5(0.3)$ & $-6.5(0.7)$ & -12.1 & -54.6 \\
\hline
\end{tabular}

* Angular change as a percent of the corresponding average ROM of straight step.

Table 4.1 shows a modest decrease of ROM in DP direction during the step turn compared to the straight step. The ROM in the IE direction increased by $23.8 \%$, 
indicating the significance of the IE role during turning. A significantly smaller ROM in EI may suggest a higher stiffness in that axis of rotation necessary to transfer the reaction forces from the ground to the body. As the step progressed through the gait cycle, noticeable differences were observed between the straight step and step turn for all subjects.

Table 4.2 shows the average ankle rotations in straight and turning steps at different phases of stance periods. During a step turn, IE had the largest deviation from the ankle rotations in the straight step. During the step turn, IE started with $5.9^{\circ}$ of inversion and increased to $13.6^{\circ}$ at push off, suggesting a gradual increase in inversion to lean the body toward the inside of the turn. This was significantly different from straight step that started at $1.7^{\circ}$ eversion at heel strike and transitioned to $1.4^{\circ}$ inversion at push off. These results indicated that the change in ankle angle in the IE direction at the step turn is significantly larger and different from straight step (19). The ankle inversion is required for generating a ground reaction force during the step turn as reported in $(18,36)$. DP displacement started at a similar initial angle as the straight step at the heel strike $\left(-9.7^{\circ}\right.$ of dorsiflexion) but progressively showed less plantarflexion at push off $\left(1.4^{\circ}\right.$ in step turn compared to $10.4^{\circ}$ in straight walk). At the heel strike of the step turn, EI displacement had an increase of $5.7^{\circ}$ of medial rotation compared to straight walk that may suggest an anticipatory motion of the foot. The difference in lateral rotation during straight step and step turn at the push off increased to $12.1^{\circ}$.

\subsubsection{AnKLe Rotations Step Turn at Different Gait SPEedS}

In a second set of experiments, the step turn maneuvers at two different gait speeds were studied and the results were compared to the ankle rotations in straight steps in both left and right ankles. Seven young subjects were participated in this set of experiment. The slow speed was set to 96 steps per minute synchronized to an audible metronome. The fast speed was different among the participants with an average of 114 steps per minute, calculated from the right foot data. The subjects 
were instructed to go as fast as they felt comfortable to perform the step turn without occurrence of a flight phase (37). The step turn experiments at both speeds were performed with similar protocol to the previous experiment. Each experiment was repeated five times for each subject and the results were averaged across the trials. The average angular rotations of each stride segment were calculated across the participants' data. The percent change in IE direction were greater than the other two DOF, confirming the results from the previous experiment.

Table 4.3 shows the average percent change of the ankle rotations in the stance period of right and left ankles during a step turn compared to straight steps at different gait speeds. The step turn initiated on the right foot to redirect the body to the left. It was seen that the IE motion of the right ankle for the straight steps in both slow and fast speed at the corresponding phases are close. However, the deviation of the right ankle IE during the turn was increased significantly with the speed. Specifically, the push off at straight step and low speed showed a $2.8^{\circ}$ eversion, while during the turn, it changed to $10.5^{\circ}$ inversion. During the fast speed, these values were $2.5^{\circ}$ eversion and $14.3^{\circ}$ inversion, respectively. Those values were equivalent of $248 \%$ and $312 \%$ deviations (as a percent of the straight step IE average ROM) from the straight step rotation in IE for the slow and fast speed tests, respectively. Similar trends were observed for heel strike and flat foot of the right ankle.

\subsection{CONCLUSION}

Human ankle rotation during step turn at two different speeds were measured using a camera system. It was shown that the rotation of the ankle in inversioneversion significantly changed during this gait maneuver when compared to straight walking. The results implied that a multi-axis ankle-foot prosthesis could increase the agility of the gait by mimicking the ankle kinematics, which may improve turning and the quality of life of below knee amputees. 
Table 4.3. Bilateral ankle ROM in IE direction during stance for a step turn compared to straight steps at slow and fast speeds.

\begin{tabular}{|c|c|c|c|}
\hline & $\begin{array}{c}\text { Right Ankle } \\
\text { Straight Step, } \\
\text { Slow } \\
\text { mean (std. error) }\end{array}$ & $\begin{array}{c}\text { Right Ankle } \\
\text { Turning Step, } \\
\text { Slow } \\
\text { mean (std. error) }\end{array}$ & $\begin{array}{c}\text { Angular Change } \\
\text { as a Percent of } \\
\text { ROM }^{*}\end{array}$ \\
\hline heel strike & $-2.3(0.8)$ & $7.8(0.9)$ & 180 \\
\hline mid stance & $-3.4(0.7)$ & $7.3(0.8)$ & 199 \\
\hline \multirow[t]{2}{*}{ push off } & $-2.8(0.7)$ & $10.6(1.3)$ & 248 \\
\hline & $\begin{array}{c}\text { Right Ankle } \\
\text { Straight Step, } \\
\text { Fast }\end{array}$ & $\begin{array}{c}\text { Right Ankle } \\
\text { Turning Step, } \\
\text { Fast }\end{array}$ & \\
\hline heel strike & $-2.5(0.9)$ & $9.9(0.9)$ & 231 \\
\hline mid stance & $-3.1(0.7)$ & $10.8(0.7)$ & 259 \\
\hline \multirow[t]{2}{*}{ push off } & $-2.5(0.8)$ & $14.3(1.0)$ & 312 \\
\hline & $\begin{array}{c}\text { Left Ankle } \\
\text { Straight Step, } \\
\text { Slow }\end{array}$ & $\begin{array}{l}\text { Left Ankle } \\
\text { Turning Step, } \\
\text { Slow } \\
\end{array}$ & \\
\hline heel strike & $-4.8(1.2)$ & $-7.2(2.0)$ & -45 \\
\hline mid stance & $-7.3(1.3)$ & $-8.0(1.4)$ & -13 \\
\hline \multirow[t]{2}{*}{ push off } & $-9.7(1.6)$ & $-8.5(1.4)$ & 22 \\
\hline & $\begin{array}{c}\text { Left Ankle } \\
\text { Straight Step, } \\
\text { Fast }\end{array}$ & $\begin{array}{l}\text { Left Ankle } \\
\text { Turning Step, } \\
\text { Fast }\end{array}$ & \\
\hline heel strike & $-5.0(1.2)$ & $-8.5(1.6)$ & -66 \\
\hline mid stance & $-7.8(1.5)$ & $-9.1(1.5)$ & -24 \\
\hline push off & .8) & (1.3) & 20 \\
\hline
\end{tabular}

* Angular change as a percent of the corresponding average ROM of straight step 


\section{5-ANKLE MECHANICS DURING SIDESTEP CUTTING IMPLICATES NEED FOR 2-DOF POWERED ANKLE- FOOT PROSTHESES*}

Evandro M. Ficanha, Mohammad Rastgaar, Kenton R. Kaufman

\subsection{ABSTRACT}

The ankle joint of currently available powered prostheses are capable of controlling one degree of freedom (DOF), focusing on improved mobility in the sagittal plane. To increase agility, the requirements of turning in the design of prostheses need to be considered.

Ankle kinematics and kinetics were studied during sidestep cutting and straight walking. There were no significant differences between the ankle sagittal plane mechanics when comparing sidestep cutting and straight walking; however, significant differences were observed in ankle frontal plane mechanics. During the straight walk the inversion-eversion (IE) angles were small when compared to the sidestep cutting. The ankle that initiated the sidestep cutting, showed progressively increasing inversion from $2^{\circ}$ to $13^{\circ}$ while the following contralateral step, showed progressively decreasing inversion from $8^{\circ}$ to $-4^{\circ}$ during normal walking speed. The changes in IE kinematics were the most significant during sidestep cutting compared to straight walking. The IE moments of the step which initiated the sidestep cutting were always in eversion acting as a braking moment opposing the inverting motion. This suggests that an ankle-foot prosthesis with active DOF in sagittal and frontal planes will increase the agility of gait for patients with limb loss.

\footnotetext{
*The material contained in this chapter was previously published in the Journal of Rehabilitation Research \& Development. The permission to use the material is shown in appendix B.
} 


\section{2- INTRODUCTION}

Recent advances in powered prostheses promise to significantly improve the quality of life and well-being for individuals with impaired mobility. A better understanding of the complexities surrounding lower limb prostheses, which are needed for walking and daily activities, will lead to increased health and well-being for the 1.7 million limb amputees in the US, the majority of whom have lower extremity amputations $(2,15)$. The ankle joint of current commercially available lower extremity powered prostheses are capable of controlling only one DOF in the sagittal plane, focusing on improved mobility in straight walking. Turning, however, plays a major role in daily living activities and requires ankle control in both sagittal and frontal planes. Additionally, even during walking on a straight path, the ankle functions in both the sagittal and frontal planes. This suggests that the next advancement in prosthetic ankles is to extend their design and control to the frontal plane.

Agility describes the ability to alter the direction of the body efficiently and effectively. One definition of agility is proposed as "a rapid whole body movement with change of velocity or direction in response to a stimulus" (38). Agility requires both maneuverability and speed that are limited in lower extremity amputees who use passive prostheses. It is shown that individuals with a unilateral below-knee amputation who use passive prostheses rely more on their hip joint and expend 20$30 \%$ more metabolic energy compared to non-amputees at the same speed. As a result, their preferred speed of gait is $30-40 \%$ lower than non-amputees $(39,40)$. Additionally, amputees use compensatory strategies that result in asymmetrical gait patterns that affect joints in both lower limbs which may inadvertently lead to secondary complications such as knee or hip osteoarthritis of the intact limb or back pain (41-44). In contrast, it has been shown that a powered ankle-foot prosthesis reduces the metabolic costs of unilateral transtibial amputees during straight walking by providing sufficient power during push-off $(33,45)$. However, studies of four representative daily activities show that turning steps may account for an 
average of $25 \%$ of steps, ranging from $8 \%-50 \%$ of all steps depending on the activity (1), which amputees accomplish using different control strategies than nonamputees. While a non-amputee relies on hip movement in the frontal plane and moments generated at the ankle, an amputee using a passive prosthesis relies on hip extension in the sagittal plane $(6,7,16,17)$. During a turn, modulation of ankle impedance in the sagittal and frontal planes plays a major role in controlling lateral and propulsive ground reaction forces in order to accelerate the body center of mass along the gait path; thus, during a turning step, lateral and propulsive impulses are larger compared to a straight step (18). This difference will result in different gait strategies between amputees and non-amputees to compensate for the lack of propulsion from a passive prosthesis in order to increase maneuverability (7). This suggests that an ankle-foot prosthesis capable of generating moments in two DOF, i.e. dorsiflexion-plantarflexion (DP) and IE directions, with impedance modulation similar to the human ankle will augment maneuverability and mobility that leads to a more agile gait. Additionally, design features that allow walking in arbitrary directions on slopes while conforming the foot to the uneven ground profile may result in a more efficient gait.

Understanding the ankle's capability to modulate impedance while generating net positive work during the stance period of gait has influenced the design of new ankle-foot prosthesis $(20-22,24)$. The ankle mechanical impedance is a dynamic operator that maps the time-history of angular displacements onto the corresponding time-history of ankle moments. While these prostheses have advanced the state-ofthe-art design, they are specifically designed for different gait scenarios in the sagittal plane. The design strategy may be improved by incorporating an additional DOF, considering that even level walking in a straight line requires the ankle to function in both the sagittal and frontal planes. Additionally, normal daily activity includes more gait scenarios such as turning, traversing slopes, and adapting to uneven terrain profiles. To extend the design of ankle-foot control to the frontal 
plane, a better understanding of the multi-variable mechanical impedance of the human ankle is needed.

Ankle mechanical impedance may provide an opportunity to better characterize ankle dynamics. Mechanical impedance of a dynamic system determines the evoked moment due to input motion perturbations and is a function of the stiffness, viscoelasticity, and inertia of the system. The ankle's mechanical impedance in a single DOF has been the focus of all prior studies, while multidirectional ankle characteristics have not been studied. Additionally, single DOF ankle movements are rare in normal lower limb actions, so control of multiple ankle DOF presents unique challenges (46). Therefore, understanding the ankle directional impedance during gait is a key factor for improving the design of lower extremity prostheses.

Powered prostheses controllers are currently designed based on ankle momentangle relationships that are averaged across a study population (e.g. see Shamaei et al. (47)) rather than ankle impedance. Rouse et al. developed a platform capable of applying moment perturbations during the foot-flat stance phase in sagittal plane $(48,49)$. Mechanical ankle impedance in both DP and IE directions in non-load bearing conditions and stationary conditions was estimated by Rastgaar et al. (50, 51) for dynamic mechanical impedance and Lee et al. (52-55) for quasi-static mechanical impedance. Ho et al. also studied the directional variation of quasi-static ankle mechanical impedance $(56,57)$. Further, Lee et al. developed a method for estimation of time-varying mechanical impedance of ankle during the entire stride length for the subjects walking on a treadmill (58). Their study on unimpaired subjects showed consistent time-varying characteristics of ankle impedance during the entire stride in both sagittal and frontal planes.

In this paper, ankle displacements and moments were studied during straight walking and sidestep cutting. The goal of this study is to show how the kinematics and kinetics of the ankle, and therefore its mechanical impedance, change in different maneuvers. The term sidestep cutting is used to describe the motion of 
pushing the body sideways using the leading leg to translate the body while walking forward (the motion is at or near 45 degrees from the original path) without rotating the body (e.g. stepping sideways to avoiding an obstacle on the ground). The paper describes experiments for collecting ankle kinematics and kinetics in the sagittal, frontal, and transverse planes during sidestep cutting and comparing the results with walking along a straight path.

\section{3- ANKLE KINEMATICS AND KINETICS DURING GAIT}

To change direction during gait, one needs to perform different gait maneuvers such as a step turn, spin turn, or sidestep cutting. These maneuvers have different kinematics and kinetics. For example, compared to straight walking, step turns have considerably different velocity, length, width, and higher turning reaction forces $(6$, $15,17,18)$. Also the ankle moment in the inversion direction is significantly different from the straight steps and spin turn steps (35).

A series of experiments were performed to quantify the ankle kinematics and kinetics behavior in the context of gait agility. The experiments measured the ankle kinematics and kinetics during stance phase of the sidestep cutting and compared the results to the ankle mechanics during straight walking. The study did not include any cognitive aspect of agility, but focused on the ankle kinematics and kinetics due to change of direction and speed.

Five male subjects with no self-reported neuromuscular and biomechanical disorders were recruited for the experiments (ages from 23 to 27 years and body mass index from 23 to $28 \mathrm{~kg} / \mathrm{m}^{2}$ ). The subjects gave written consent to participate in the experiment which was approved by the Michigan Technological University Institutional Review Board.

\section{4- EXPERIMENT SETUP AND ANALYSIS}

Hansen et al. described ankle moments in the sagittal plane during straight walking (24). To calculate the ankle kinetics during walking, it was necessary to 
estimate the location of ankle center of rotation, the reaction forces, and the moment arms for the reaction forces. The ankle is composed of the talocrural and the subtalar joints. It has been shown that the combined movement of both joints can be approximated as a monocentric single DOF hinge joints for functional activities such as walking and running (59). To identify the ankle center of rotation, the recommended definition by the International Society of Biomechanics (ISB) was used (60). External markers were required to identify each joint as it is not possible to directly discern between the talocrural and the subtalar joints. The ankle center of rotation was approximated as the midpoint between the tip of the medial malleolus and the tip of the lateral malleolus for DP and external-internal rotations (EI). The approximation is also valid for IE at ankle neutral position when DP, IE, and EI angles are all zeroes (60). In this manuscript, dorsiflexion, inversion, and internal rotations were defined as positive rotations, similar to ISB definitions. Similarly, plantarflexion, eversion, and external rotations were defined as negative rotations. This notation was used throughout the paper, where DP, IE, and EI are the foot rotations about the $\mathrm{X}, \mathrm{Y}$, and $\mathrm{Z}$ axis of the foot coordinate system, respectively.

A motion capture camera system was used to track the foot rotations and the position of the ankle center. The motion capture camera system consisted of eight Prime $17 \mathrm{~W}$ Optitrack ${ }^{\circledR}$ cameras in a square formation covering a volume of about 16 cubic meters and an area of 12 square meters. The cameras emitted infrared light and captured the reflected light from reflectors mounted on the participants at a rate of $300 \mathrm{~Hz}$. Two reflective markers were placed on the participant, one at the tip of the medial malleolus and the other at the tip of the lateral malleolus. The markers' positions were recorded during the test, and the ankle center of rotation was estimated as the midpoint of the two markers in the global reference frame. Reflective markers were attached to two polycarbonate plastic rigid bodies developed by the camera system manufacturer to eliminate relative motion of the markers with respect to each other. One of the polycarbonate rigid bodies was attached to the participant's shoe above the metatarsal bones to record the global 
foot rotations. The second polycarbonate rigid body was attached to the participant's shin to record the global position of the shin. The ankle rotations were calculated as the relative rotations of the foot with respect to the shin.

Sidestep cutting maneuvers were initiated on the right foot. Two different gait speeds were studied and the results were compared to the straight steps for both left and right ankles. The slow speed was the preferred speed of gait of the participant (average of 96 steps per minute). In the fast speed tests, the subjects were instructed to go as fast as they felt comfortable to perform sidestep cutting without occurrence of a flight phase (average of 114 steps per minute) (37). The participants were instructed to start walking in a straight path from the outside of the field of view of the cameras. When they reached an obstacle on the ground, they performed a sidestep cutting to the left, pivoting about their right leg to avoid the obstacle and switched direction immediately; followed by a left leg sidestep cutting that redirected the walk in a straight line parallel to the initial direction of gait (Fig. 5.1). Similarly, straight walking tests were performed at slow and fast speed, where each subject walked on a straight line stepping on the force plate. The straight walk tests were repeated for measuring the ground reaction forces for both the right and left foot at both slow and fast speeds (Fig. 5.2A and 5.2B, respectively). Each of the four different steps (left and right legs at straight walk and sidestep cutting) were repeated five times for each subject at both slow and fast speeds.

To measure the ground reaction forces, a Kistler ${ }^{\circledR}$ Type 5233A force plate was used. The ground reaction forces in the $\mathrm{X}, \mathrm{Y}$ and $\mathrm{Z}$ global axis (approximately pointing to the right, forward, and cephalad of the foot, respectively) were obtained directly from the force plate. The $\mathrm{Z}$ axis force, which was obtained from four individual load cells at each corner of the force plate, was also used to identify the location of the center of pressure of the foot when placed on the force plate. The origin of the force plate coordinate system was also the origin of the global coordinate system of the camera system. During the experiments, the data from the force plate was collected at 300 samples per second. 


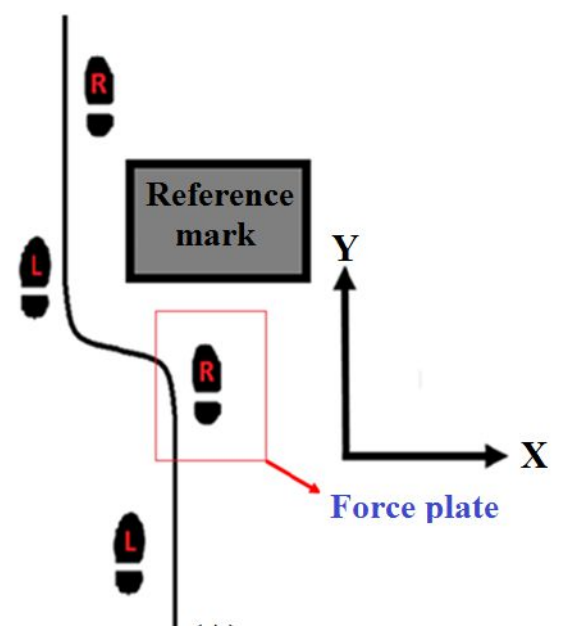

(A)

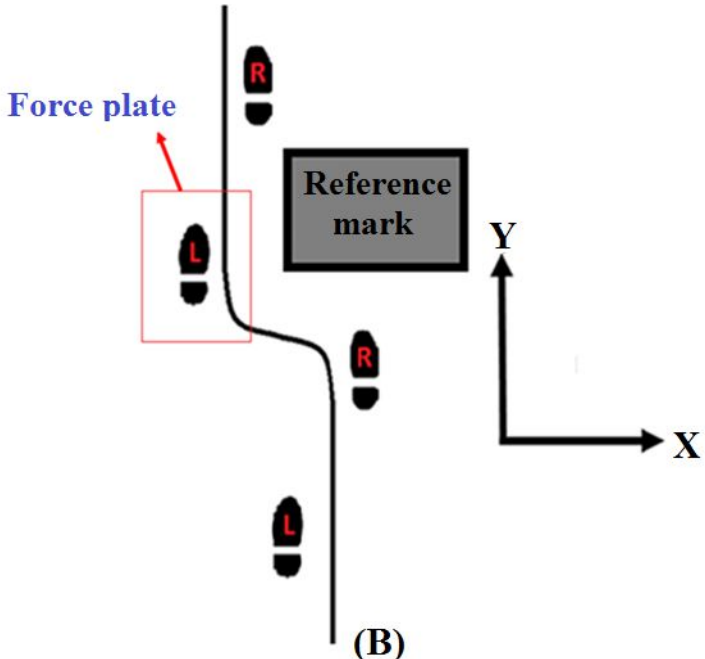

(B)

Figure 5.1. Foot position during sidestep cutting. (A): To record the right foot ground reaction moments and ankle angles. (B): To record the left foot ground reaction moments and ankle angles.
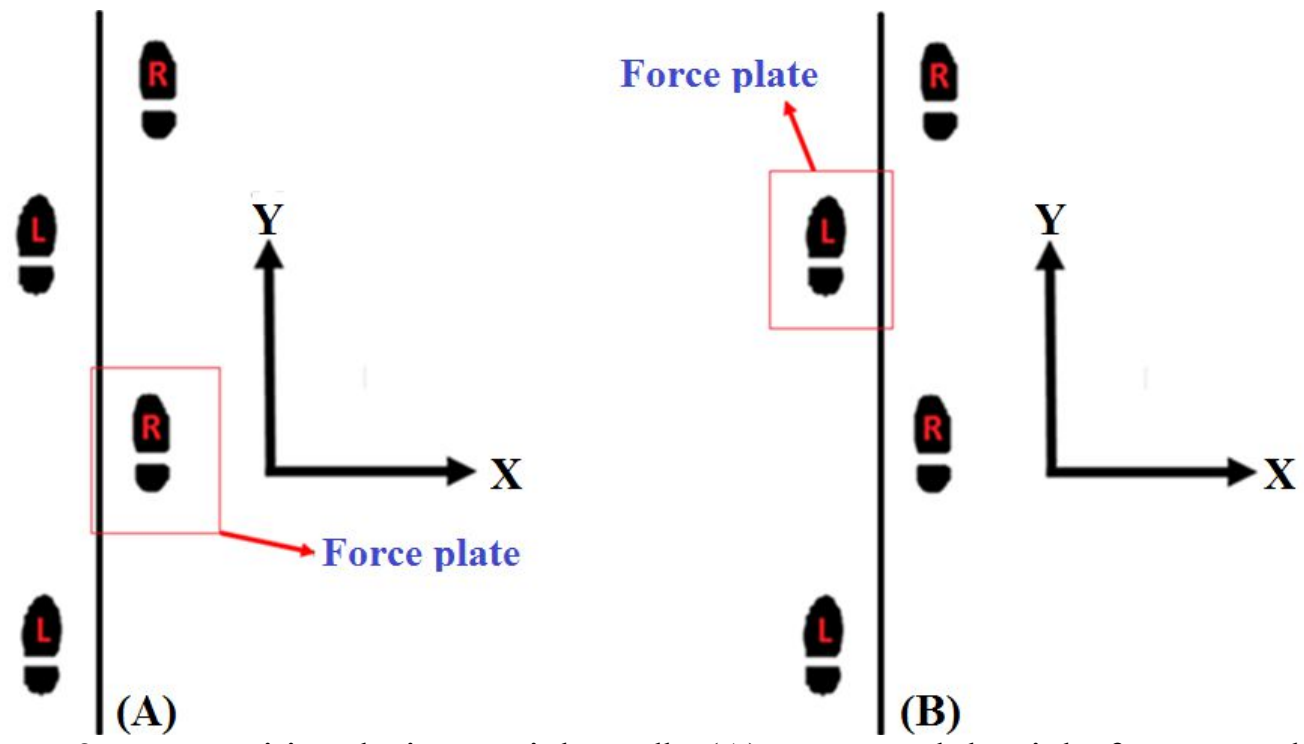

Figure 5.2. Foot position during straight walk. (A): To record the right foot ground reaction moments and ankle angles. (B): To record the left foot ground reaction moments and ankle angles.

The distances between the ankle center of rotation and the center of pressure in the global coordinates, which were necessary for the estimation of the moment arms, were obtained directly from the markers positions in the global coordinate 
system. The displacements and the forces obtained in the global reference frame were transformed to the foot coordinate system using a rotation matrix. The rotation matrix was defined using the Euler angles describing the rotation of the foot (obtained from the markers placed on the rigid body on the subject's foot) relative to the global reference frame. Once the displacements and forces were transformed to the foot coordinate system, the normalized moments (with respect to the subjects' body mass) were calculated. A view of the ground reaction forces and moment arms for calculations of the ankle moments in DP, IE, and EI can be seen in Fig. 5.3 A, B, and $\mathrm{C}$, respectively.

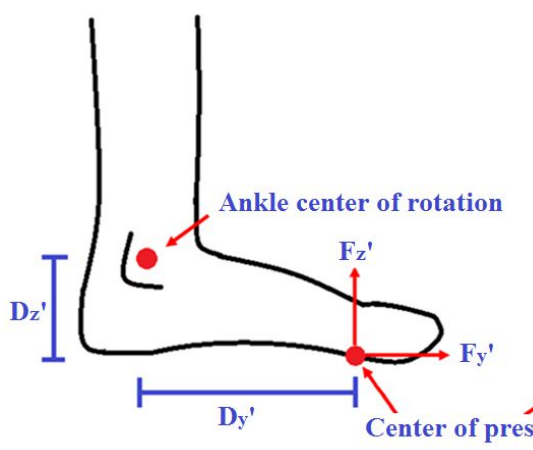

(A)

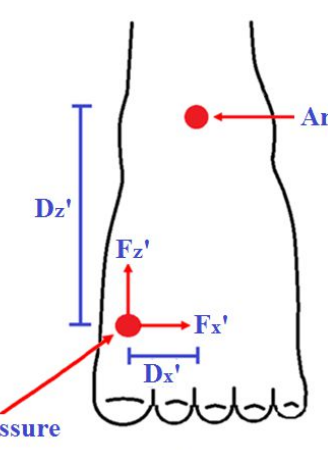

(B)

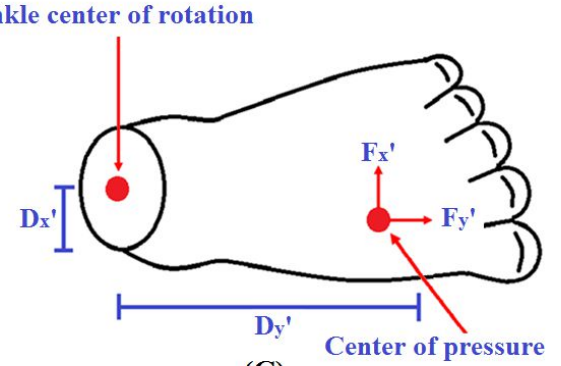

(C)

Figure 5.3. (A): Foot schematics in the sagittal plane showing ground reaction forces and moment arms for moment estimation in DP. (B): Foot schematics in the frontal plane showing ground reaction forces and moment arms for moment estimation in IE. (C): Foot schematics in the transverse plane showing ground reaction forces and moment arms for moment estimation in EI.

Three phases of the stance were identifiable using the data from the cameras and force plate. The weight acceptance (WA) phase started when the heel contacted the floor up to the point where the whole area of the foot is in contact with the floor. The mid-stance (MS) was identified as the entire duration that the foot is in full contact with the floor. The terminal stance and pre-swing (TP) phase was identified from the point where the heel stops contacting the floor to the point where there is no contact between the foot and the floor. The subjects were weighed on the day of the test using the force plate and the moments were normalized with respect to the 
subject's weight. The moments and ankle angles at each stance period were calculated from the walking trials. The averages and standard errors of the angles and moments of the 25 entries for each step type (each of the 5 participants performed 5 trials of each step type) were calculated. The changes in average moment and angle between the straight step and sidestep cutting tests were calculated at each phase of stance period (WA, MS, and TP). One-way analysis of variance (ANOVA) was used to compare the straight to turning step results to show if their differences were statistically significant $(p<0.05)$.

\section{5- RESULTS AND DISCUSSION}

\subsection{1- KINEMATICS}

\subsubsection{1- Sagittal Plane Kinematics}

No evident differences were observed in ankle kinematics in the sagittal plane (Fig. 5.4). Tables 5.1-5.4 all show that there was no statistically significant difference between the right ankle kinematics in the sagittal plane during the different stance phases for straight steps and cutting steps at either slow or fast speeds.

Table 5.1. Right ankle normalized moments and angles during stance period of straight walk at slow speed and sidestep cutting at slow speed. Positive angle are dorsiflexion, inversion, and internal rotation for DP, IE and EI, respectively.

\begin{tabular}{|c|c|c|c|c|c|c|c|c|c|c|c|c|c|}
\hline \multirow{2}{*}{\multicolumn{2}{|c|}{ Right Slow }} & \multicolumn{2}{|c|}{$\begin{array}{c}\text { Torque straight } \\
\text { slow ( } \mathrm{Nm} / \mathrm{kg} \text { ) }\end{array}$} & \multicolumn{2}{|c|}{$\begin{array}{l}\text { Torque cutting } \\
\text { slow ( } \mathrm{Nm} / \mathrm{kg})\end{array}$} & \multirow{2}{*}{\begin{tabular}{|c|}
$\begin{array}{c}\text { Change } \\
\text { in }\end{array}$ \\
torque \\
$(\mathrm{Nm} / \mathrm{kg})$ \\
\end{tabular}} & \multirow{2}{*}{$\begin{array}{l}\text { Torque } \\
\mathrm{p} \text {-Value }\end{array}$} & \multicolumn{2}{|c|}{$\begin{array}{l}\text { Angle straight } \\
\text { Slow (degrees) }\end{array}$} & \multicolumn{2}{|c|}{$\begin{array}{l}\text { Angle cutting } \\
\text { Slow (degrees) }\end{array}$} & \multirow{2}{*}{$\begin{array}{c}\text { Change } \\
\text { in } \\
\text { angle } \\
\text { (degrees) }\end{array}$} & \multirow{2}{*}{$\begin{array}{l}\text { Angle } \\
\text { p-Value }\end{array}$} \\
\hline & & Mean & $\begin{array}{c}\begin{array}{c}\text { Standard } \\
\text { error }\end{array} \\
\end{array}$ & Mean & $\begin{array}{c}\text { Standard } \\
\text { error }\end{array}$ & & & Mean & $\begin{array}{c}\begin{array}{c}\text { Standard } \\
\text { error }\end{array} \\
\end{array}$ & Mean & $\begin{array}{c}\text { Standard } \\
\text { error }\end{array}$ & & \\
\hline \multirow{3}{*}{ DP } & WA & 0.31 & 0.03 & 0.33 & 0.03 & 0.02 & 0.65 & -3 & 1.2 & -3 & 1.4 & 0 & 0.98 \\
\hline & MS & $\mid-0.67$ & 0.02 & -0.56 & 0.04 & 0.11 & 0.06 & 3 & 0.8 & 4 & 0.9 & 1 & 0.40 \\
\hline & $\mathrm{TP}$ & -1.03 & 0.04 & -1.07 & 0.03 & -0.04 & 0.36 & 4 & 0.8 & 4 & 0.7 & 0 & 0.74 \\
\hline \multirow{3}{*}{ IE } & WA & \begin{tabular}{|l|}
-0.04 \\
\end{tabular} & 0.01 & -0.11 & 0.02 & -0.08 & $\underline{0.00}$ & -1 & 0.8 & 4 & 0.6 & 5 & $\underline{0.00}$ \\
\hline & $\mathrm{MS}$ & -0.19 & 0.02 & -0.29 & 0.03 & -0.10 & $\underline{0.00}$ & -5 & 0.3 & 4 & 0.6 & 9 & $\underline{0.00}$ \\
\hline & TP & -0.16 & 0.02 & -0.15 & 0.04 & 0.01 & 0.86 & -3 & 0.5 & 11 & 1.0 & 14 & $\underline{0.00}$ \\
\hline \multirow{3}{*}{ EI } & WA & -0.01 & 0.00 & 0.02 & 0.00 & 0.03 & $\underline{0.00}$ & -3 & 0.8 & -5 & 0.7 & -1 & 0.21 \\
\hline & MS & -0.02 & 0.00 & -0.05 & 0.00 & -0.03 & $\underline{0.00}$ & -3 & 0.9 & -1 & 1.0 & 2 & 0.13 \\
\hline & $\mathrm{TP}$ & \begin{tabular}{|l}
-0.04 \\
\end{tabular} & 0.00 & -0.25 & 0.01 & -0.21 & 0.00 & 1 & 0.9 & 2 & 1.3 & 0.4 & 0.81 \\
\hline
\end{tabular}


Table 5.2. Right ankle normalized moments and angles during stance period of straight walk at fast speed and sidestep cutting at fast speed. Positive angle are dorsiflexion, inversion, and internal rotation for DP, IE and EI, respectively.

\begin{tabular}{|c|c|c|c|c|c|c|c|c|c|c|c|c|c|}
\hline \multirow{2}{*}{\multicolumn{2}{|c|}{ Right Fast }} & \multicolumn{2}{|c|}{$\begin{array}{c}\text { Torque straight } \\
\text { fast }(\mathrm{Nm} / \mathrm{kg})\end{array}$} & \multicolumn{2}{|c|}{$\begin{array}{l}\text { Torque cutting } \\
\text { fast }(\mathrm{Nm} / \mathrm{kg})\end{array}$} & \multirow{2}{*}{$\begin{array}{c}\text { Change } \\
\text { in } \\
\text { torque } \\
(\mathrm{Nm} / \mathrm{kg})\end{array}$} & \multirow{2}{*}{$\begin{array}{l}\text { Torque } \\
\text { p-Value }\end{array}$} & \multicolumn{2}{|c|}{$\begin{array}{l}\text { Angle straight } \\
\text { fast }(\mathrm{Nm} / \mathrm{kg})\end{array}$} & \multicolumn{2}{|c|}{$\begin{array}{l}\text { Angle cutting } \\
\text { fast }(\mathrm{Nm} / \mathrm{kg})\end{array}$} & \multirow{2}{*}{$\begin{array}{c}\text { Change } \\
\text { in } \\
\text { angle } \\
\text { (degrees) }\end{array}$} & \multirow{2}{*}{$\begin{array}{c}\text { Angle } \\
\text { p-Value }\end{array}$} \\
\hline & & Mean & \begin{tabular}{|c|}
$\begin{array}{c}\text { Standard } \\
\text { error }\end{array}$ \\
\end{tabular} & Mean & $\begin{array}{c}\text { Standard } \\
\text { error }\end{array}$ & & & Mean & $\begin{array}{c}\text { Standard } \\
\text { error }\end{array}$ & Mean & $\begin{array}{c}\text { Standard } \\
\text { error }\end{array}$ & & \\
\hline \multirow{3}{*}{ DP } & WA & 0.64 & 0.04 & 0.53 & 0.05 & -0.10 & 0.10 & 2 & 1.5 & 1 & 1.3 & 0 & 0.82 \\
\hline & MS & -0.47 & 0.03 & -0.42 & 0.03 & 0.05 & 0.24 & 4 & 0.8 & 6 & 0.7 & 2 & 0.06 \\
\hline & TP & -1.15 & 0.04 & -1.16 & 0.04 & -0.01 & 0.80 & 0 & 0.9 & 2 & 1.2 & 2 & 0.20 \\
\hline \multirow{3}{*}{$\mathrm{IE}$} & WA & -0.05 & 0.02 & -0.21 & 0.02 & -0.15 & $\underline{0.00}$ & -3 & 0.8 & 5 & 0.7 & 8 & $\underline{0.00}$ \\
\hline & $\mathrm{MS}$ & -0.18 & 0.01 & -0.31 & 0.02 & -0.13 & $\underline{0.00}$ & -4 & 0.4 & 6 & 0.5 & 10 & $\underline{0.00}$ \\
\hline & TP & -0.17 & 0.02 & -0.15 & 0.03 & 0.03 & 0.51 & -4 & 0.6 & 10 & 0.7 & 14 & $\underline{0.00}$ \\
\hline \multirow{3}{*}{ EI } & WA & -0.01 & 0.01 & 0.06 & 0.01 & 0.07 & $\underline{0.00}$ & -4 & 0.8 & -5 & 0.8 & -1 & 0.49 \\
\hline & $\mathrm{MS}$ & -0.01 & 0.00 & -0.04 & 0.00 & -0.03 & $\underline{0.00}$ & -4 & 0.7 & -2 & 0.9 & 2 & 0.05 \\
\hline & TP & -0.05 & 0.01 & -0.28 & 0.01 & -0.23 & $\underline{0.00}$ & 2 & 0.8 & 2 & 1.3 & 0 & 0.95 \\
\hline
\end{tabular}

Table 5.3. Left ankle normalized moments and angles during stance period of straight walk at slow speed and sidestep cutting at slow speed. Positive angle are dorsiflexion, inversion, and internal rotation for DP, IE and EI, respectively.

\begin{tabular}{|c|c|c|c|c|c|c|c|c|c|c|c|c|c|}
\hline \multirow{2}{*}{\multicolumn{2}{|c|}{ Left Slow }} & \multicolumn{2}{|c|}{$\begin{array}{c}\text { Torque straight } \\
\text { slow (Nm/kg) }\end{array}$} & \multicolumn{2}{|c|}{$\begin{array}{l}\text { Torque cutting } \\
\text { slow (Nm/kg) }\end{array}$} & \multirow{2}{*}{$\begin{array}{c}\text { Change } \\
\text { in } \\
\text { torque } \\
(\mathrm{Nm} / \mathrm{kg})\end{array}$} & \multirow[t]{2}{*}{$\begin{array}{l}\text { Torque } \\
p \text {-Value }\end{array}$} & \multicolumn{2}{|c|}{$\begin{array}{l}\text { Angle straight } \\
\text { Slow (degrees) }\end{array}$} & \multicolumn{2}{|c|}{$\begin{array}{l}\text { Angle cutting } \\
\text { Slow (degrees) }\end{array}$} & \multirow{2}{*}{$\begin{array}{l}\text { Change } \\
\text { in } \\
\text { angle } \\
\text { (degrees) }\end{array}$} & \multirow[t]{2}{*}{$\begin{array}{c}\text { Angle } \\
p \text {-Value }\end{array}$} \\
\hline & & Mean & $\begin{array}{c}\text { Standard } \\
\text { error }\end{array}$ & Mean & $\begin{array}{c}\text { Standard } \\
\text { error }\end{array}$ & & & Mean & $\begin{array}{c}\text { Standard } \\
\text { error }\end{array}$ & Mean & $\begin{array}{c}\text { Standard } \\
\text { error }\end{array}$ & & \\
\hline \multirow{3}{*}{ DP } & WA & 0.26 & 0.02 & 0.33 & 0.02 & 0.06 & $\underline{0.03}$ & -3 & 0.7 & -1 & 0.9 & 2 & 0.10 \\
\hline & MS & -0.68 & 0.03 & -0.62 & 0.06 & 0.06 & 0.34 & 3 & 0.7 & 5 & 0.8 & 2 & 0.09 \\
\hline & TP & -1.07 & 0.03 & -1.12 & 0.03 & -0.05 & 0.29 & 3 & 1.1 & 1 & 1.7 & -2 & 0.32 \\
\hline \multirow{3}{*}{ IE } & WA & -0.07 & 0.01 & -0.05 & 0.02 & 0.01 & 0.61 & 2 & 1.0 & 8 & 0.8 & 6 & $\underline{0.00}$ \\
\hline & MS & -0.14 & 0.02 & -0.21 & 0.02 & -0.07 & $\underline{0.01}$ & -1 & 0.7 & 3 & 0.5 & 5 & $\underline{0.00}$ \\
\hline & TP & -0.08 & 0.02 & -0.13 & 0.02 & -0.05 & 0.11 & -1 & 0.5 & 0 & 0.6 & 1 & 0.27 \\
\hline \multirow{3}{*}{ El } & WA & 0.00 & 0.00 & 0.04 & 0.00 & 0.04 & $\underline{0.00}$ & -5 & 0.4 & -8 & 0.6 & -2 & 0.00 \\
\hline & MS & -0.02 & 0.00 & -0.04 & 0.01 & -0.02 & $\underline{0.01}$ & -5 & 0.7 & -3 & 0.8 & 1 & 0.32 \\
\hline & TP & -0.04 & 0.01 & -0.06 & 0.01 & -0.02 & 0.09 & 2 & 0.7 & 4 & 0.7 & 1 & 0.14 \\
\hline
\end{tabular}

Table 5.4. Left ankle normalized moments and angles during stance period of straight walk at fast speed and sidestep cutting at fast speed. Positive angle are dorsiflexion, inversion, and internal rotation for DP, IE and EI, respectively.

\begin{tabular}{|c|c|c|c|c|c|c|c|c|c|c|c|c|c|}
\hline \multirow{2}{*}{\multicolumn{2}{|c|}{ Left Fast }} & \multicolumn{2}{|c|}{$\begin{array}{l}\text { Torque straight } \\
\text { fast }(\mathrm{Nm} / \mathrm{kg})\end{array}$} & \multicolumn{2}{|c|}{$\begin{array}{l}\text { Torque cutting } \\
\text { fast }(\mathrm{Nm} / \mathrm{kg})\end{array}$} & \multirow{2}{*}{$\begin{array}{c}\text { Change } \\
\text { in } \\
\text { torque } \\
(\mathrm{Nm} / \mathrm{kg})\end{array}$} & \multirow{2}{*}{$\begin{array}{l}\text { Torque } \\
\mathrm{p} \text {-Value }\end{array}$} & \multicolumn{2}{|c|}{$\begin{array}{l}\text { Angle straight } \\
\text { fast }(\mathrm{Nm} / \mathrm{kg})\end{array}$} & \multicolumn{2}{|c|}{$\begin{array}{l}\text { Angle cutting } \\
\text { fast ( } \mathrm{Nm} / \mathrm{kg})\end{array}$} & \multirow{2}{*}{$\begin{array}{c}\text { Change } \\
\text { in } \\
\text { angle } \\
\text { (degrees) }\end{array}$} & \multirow{2}{*}{$\begin{array}{c}\text { Angle } \\
\text { p-Value }\end{array}$} \\
\hline & & Mean & $\begin{array}{c}\text { Standard } \\
\text { error }\end{array}$ & Mean & $\begin{array}{c}\text { Standard } \\
\text { error }\end{array}$ & & & Mean & $\begin{array}{l}\text { Standard } \\
\text { error }\end{array}$ & Mean & $\begin{array}{c}\text { Standard } \\
\text { error }\end{array}$ & & \\
\hline \multirow{3}{*}{$\mathrm{DP}$} & WA & 0.51 & 0.03 & 0.51 & 0.02 & 0.00 & 0.95 & 0 & 0.8 & 1 & 0.9 & 1 & 0.54 \\
\hline & MS & -0.51 & 0.04 & -0.58 & 0.05 & -0.07 & 0.28 & 5 & 0.7 & 7 & 0.8 & 2 & 0.05 \\
\hline & TP & -1.15 & 0.03 & -1.11 & 0.02 & 0.05 & 0.18 & 0 & 1.6 & -1 & 1.7 & -1 & 0.82 \\
\hline \multirow{3}{*}{$\mathrm{IE}$} & WA & -0.09 & 0.02 & -0.11 & 0.02 & -0.01 & 0.63 & 2 & 1.0 & 7 & 1.0 & 5 & $\underline{0.00}$ \\
\hline & MS & -0.12 & 0.01 & -0.22 & 0.02 & -0.10 & $\underline{0.00}$ & -1 & 0.7 & 5 & 0.7 & 6 & $\underline{0.00}$ \\
\hline & $\mathrm{TP}$ & -0.08 & 0.02 & -0.11 & 0.02 & -0.03 & 0.30 & -2 & 0.5 & 1 & 0.9 & 2 & $\underline{0.02}$ \\
\hline \multirow{3}{*}{$\mathrm{El}$} & WA & -0.01 & 0.01 & 0.08 & 0.01 & 0.08 & $\underline{0.00}$ & -6 & 0.4 & -9 & 0.6 & -3 & $\underline{0.00}$ \\
\hline & MS & -0.01 & 0.00 & -0.04 & 0.01 & -0.03 & $\underline{0.00}$ & -6 & 0.9 & -5 & 0.9 & 1 & 0.32 \\
\hline & $\mathrm{TP}$ & -0.04 & 0.01 & -0.08 & 0.02 & -0.04 & 0.02 & 2 & 0.8 & 3 & 0.9 & 1 & 0.54 \\
\hline
\end{tabular}




\subsubsection{Frontal plane kinematics}

The ankle kinematics in the frontal plane during sidestep cutting were significantly different from the straight steps at both slow and fast speeds (Fig. 5.5). During the straight walk, the ankle rotations remained near constant. On the other hand, during the sidestep cutting, the right ankle showed a progressive inversion from a minimum rotation of $2^{\circ}$ and $3^{\circ}$ for slow and fast speeds respectively, up to a maximum rotation of $13^{\circ}$, at both speeds. However, the left ankle started from near its maximum inversion of $8^{\circ}$ and $7^{\circ}$ for slow and fast speeds respectively and progressively rotated to the minimum rotation near $-4^{\circ}$ and $-3^{\circ}$ of eversion, respectively. This showed the significance of IE rotations to shift the body sideways during the sidestep cutting.

The details of the right ankle rotations during sidestep cutting and straight walking at slow speed are shown in Table 5.1. The frontal plane kinematics showed that the ankle was always in eversion during the straight walk with the largest eversion at MS. This is expected as the body shifts from side to side during walking and shows the contribution of the ankle function in IE during straight walking. On the contrary, the ankle was in inversion during cutting step. At WA, the mean right ankle rotation during straight walking was $-1^{\circ} \pm 0.8^{\circ}$ compared to $4^{\circ} \pm 0.6^{\circ}$ for a cutting step. At MS, these values were $-5^{\circ} \pm 0.3^{\circ}$ and $4^{\circ} \pm 0.6^{\circ}$, respectively. The most significant change occurred during TP, where the mean rotation of the ankle during straight walking was $-3^{\circ} \pm 0.5^{\circ}$ compared to $11^{\circ} \pm 1.0^{\circ}$ during sidestep cutting.

The results of the comparison of the different stance phases during fast speeds were close to the results of the slow speed experiment (Table 5.2). Similar to the slow speed, there were statistically significant differences in ankle rotation at WA, MS, and TP between sidestep cutting and straight step. At WA, the mean right ankle rotation during straight step was $-3^{\circ} \pm 0.8^{\circ}$ compared to $5^{\circ} \pm 0.7^{\circ}$ for cutting step. At MS, these values were $-4^{\circ} \pm 0.4^{\circ}$ and $6^{\circ} \pm 0.5^{\circ}$, respectively. The mean rotation of 
the ankle at straight step was $-4^{\circ} \pm 0.6^{\circ}$ compared to $10^{\circ} \pm 0.7^{\circ}$ during sidestep cutting. It can be seen that the ankle rotations between the slow speed and the associated fast speed phases did not change substantially. The changes in the IE angle were significant during the sidestep cutting from straight step, where the ankle, at slow speed, showed an increased inversion of $5^{\circ}$ at WA, $9^{\circ}$ at $\mathrm{MS}$, and $14^{\circ}$ at TP; at fast speed this values were $8^{\circ}, 10^{\circ}$, and $14^{\circ}$ respectively.

For the ankle rotations in the left leg, there were statistically significant differences between all the phases of straight step and cutting step at fast speed (Table 5.4). In the slow speed experiment, only MS and WA of straight and cutting steps were significantly different (Table 5.3). Similar to the right ankle results, the ankle was in inversion during all the phases of the cutting step. The maximum average inversion during cutting occurred at WA with $8^{\circ} \pm 0.8^{\circ}$ at slow speed and $7^{\circ}$ $\pm 1.0^{\circ}$ at fast speed.

\subsubsection{Transverse plane kinematics}

The TP angles peaked between $10^{\circ}$ and $15^{\circ}$ of internal rotation in all tests except the right cutting steps at both slow and fast speeds (Fig. 5.6). The peak external rotation at slow speed was $4^{\circ}$ and in fast speed was $5^{\circ}$. During the straight steps and the left leg cutting steps, the participants pivoted on top of the standing leg to align the next step directly in front of them to maintain the forward path and thus generating the observed internal rotation. During the right leg cutting steps, the left leg cutting step that follows the right leg cutting step (Fig. 5.1) was not directly in front of the subject, but offset to the left. This caused the observed external rotation at the TP of the right ankle sidestep cutting.

The transverse plane rotations at cutting steps and straight steps were not significantly different, except at the left ankle WA at both speeds. The ankle transverse plane rotation at WA and slow speed were $-5^{\circ} \pm 0.4^{\circ}$ for straight step and $8 \pm 0.6^{\circ}$ for the cutting step. At fast speed these values were $-6^{\circ} \pm 0.4^{\circ}$ for and $-9 \pm$ $0.6^{\circ}$, respectively. 


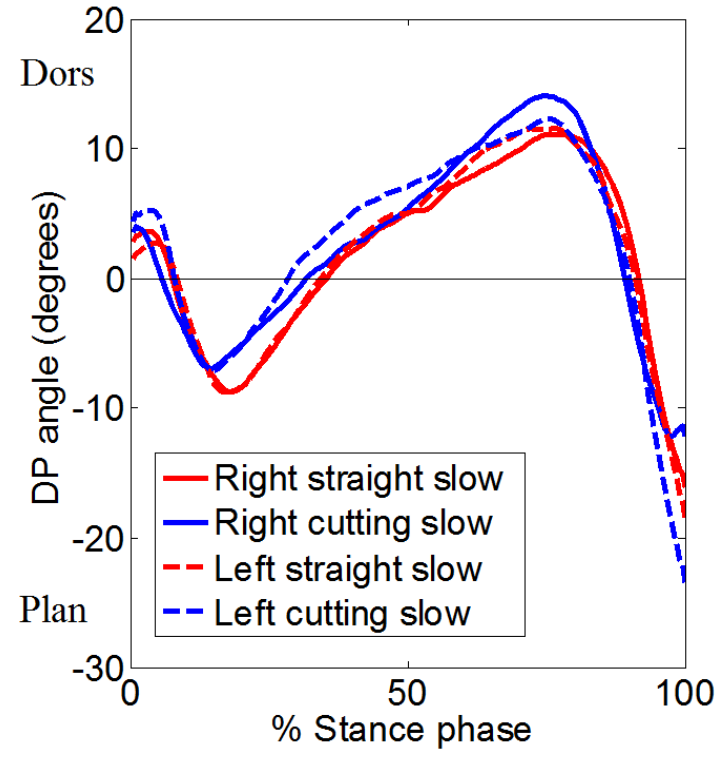

(A)

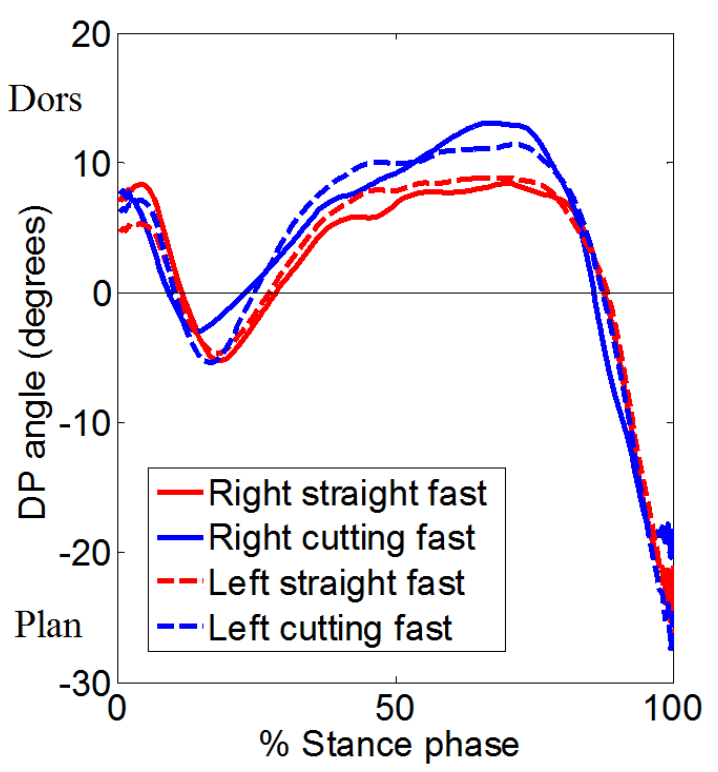

(B)

Figure 5.4. (A): Plot of the mean ankle angles in DP at slow speed. (B): Plot of the mean ankle angles in DP at fast speed. Positive angles are dorsiflexion, negative angles are plantarflexion.

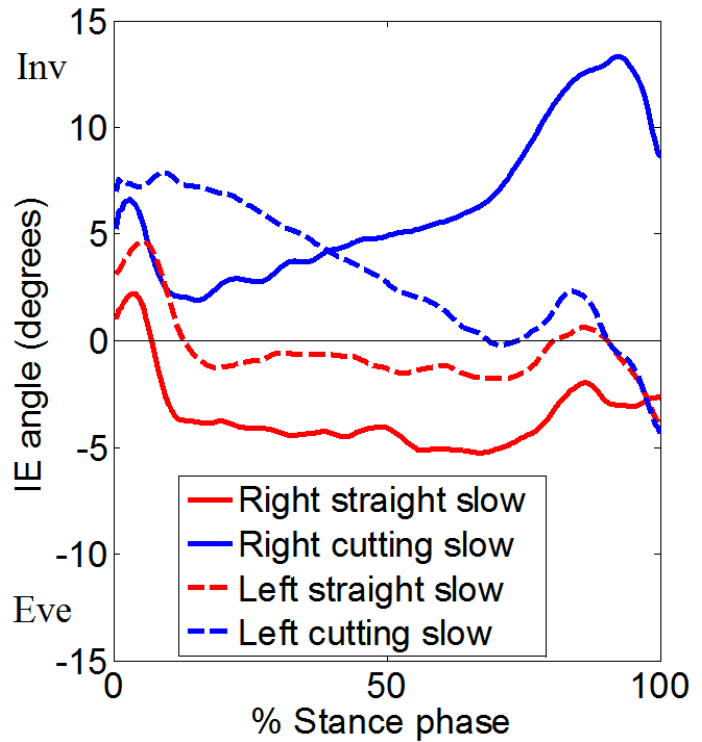

(A)

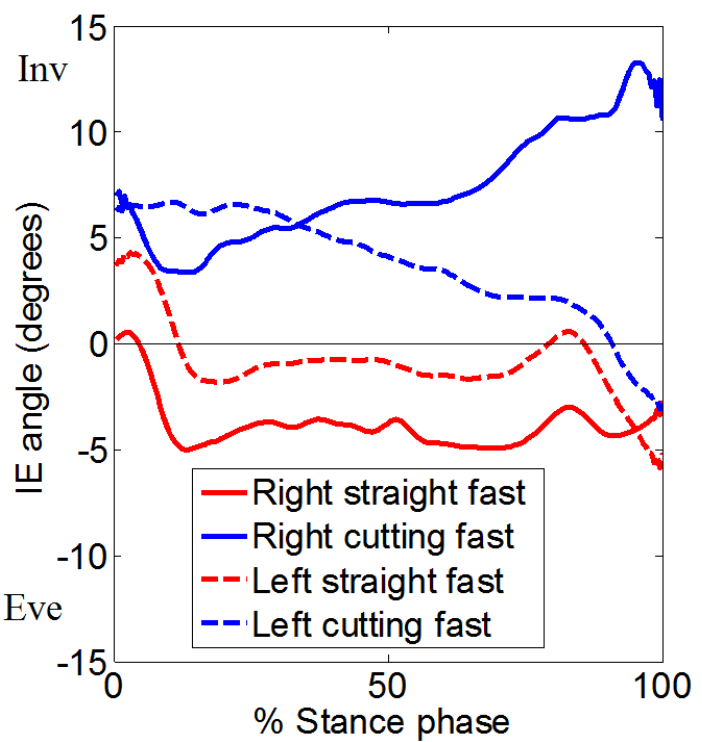

(B)

Figure 5.5. (A): Plot of the mean ankle angles in IE at slow speed. (B): Plot of the mean ankle moments in IE at fast speed. Positive angle are inversion, negative angles are eversion. 


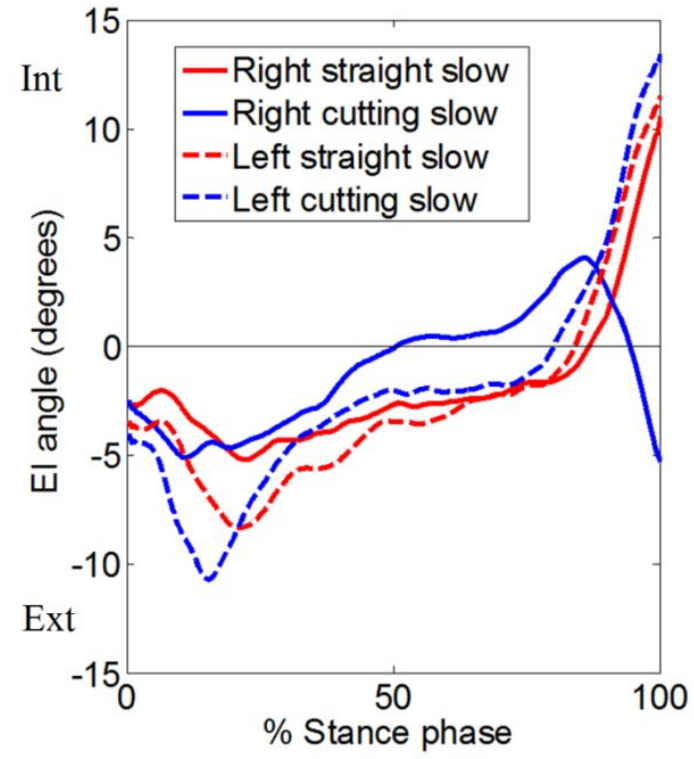

(A)

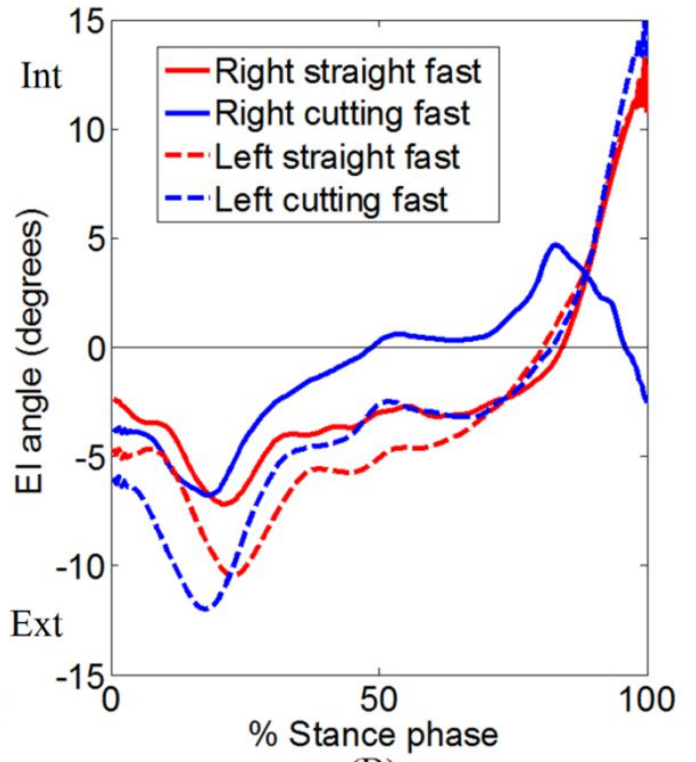

(B)

Figure 5.6. (A): Plot of the ankle angles in EI at slow speed. (B): Plot of the mean ankle angles in EI at fast speed. Positive angles are internal rotation, negative angles are external rotation.

\subsection{2- KINETICS}

\subsubsection{Sagittal plane kinetics}

The major difference among the different steps is that the dorsiflexion moment at WA was about twice as large during the fast walking tests compared to the slow walking tests, either on a straight path or during sidestep cutting (Fig 5.7). This was due to larger contact forces of the foot with the ground as expected in faster walking speeds. During MS and TP however, the differences were not as pronounced as WA. The maximum dorsiflexion moment for right ankle during sidestep cutting at slow speed was $0.47 \pm 0.04 \mathrm{Nm} / \mathrm{kg}$ and for the fast speed was $0.57 \pm 0.07 \mathrm{Nm} / \mathrm{kg}$. These values for the left ankle were $0.53 \pm 0.02 \mathrm{Nm} / \mathrm{kg}$ and $0.96 \pm 0.03 \mathrm{Nm} / \mathrm{kg}$, respectively.

During the sidestep cutting at slow speeds, the right step shifts the body to the left to avoid the obstacle on the ground. There was no statistically significant difference between DP moments at the different phases of stance period between straight walking and sidestep cutting (Table 5.1). At WA, the mean moment values 
were $0.31 \pm 0.03 \mathrm{Nm} / \mathrm{kg}$ and $0.33 \pm 0.02 \mathrm{Nm} / \mathrm{kg}$ for straight steps and cutting steps at slow speed, respectively. At MS, those values were $-0.67 \pm 0.02 \mathrm{Nm} / \mathrm{kg}$ and -0.56 $\pm 0.04 \mathrm{Nm} / \mathrm{kg}$, respectively and for TP they were $-1.03 \pm 0.04 \mathrm{Nm} / \mathrm{kg}$ and $-1.07 \pm$ $0.03 \mathrm{Nm} / \mathrm{kg}$, respectively. It was observed that the mean moment at TP was greater than MS and WA, in both sidestep cutting and straight steps.

There was no statistically significant difference between DP moments at the different phases of stance period of straight steps and cutting steps at fast speeds (Table 5.2). The maximum average moment occurred at TP, with $-1.15 \pm 0.04$ $\mathrm{Nm} / \mathrm{kg}$ at straight walk and $-1.16 \pm 0.04 \mathrm{Nm} / \mathrm{kg}$ at sidestep cutting.

During the sidestep cutting, the left step following the right foot cutting step, redirects the body to the original walking direction. There was a statistically significant difference in the sagittal plane moments at WA during straight walking and sidestep cutting at slow speed (Table 5.3). The corresponding moment values were $0.26 \pm 0.02 \mathrm{Nm} / \mathrm{kg}$ at the WA of the straight step and $0.33 \pm 0.02 \mathrm{Nm} / \mathrm{kg}$ for cutting steps. There was no statistically significant difference between sagittal plane moments at MS and TP phases of stance for straight walking compared to sidestep cutting. At MS, the mean moment values were $-0.68 \pm 0.03 \mathrm{Nm} / \mathrm{kg}$ and $-0.62 \pm 0.06$ $\mathrm{Nm} / \mathrm{kg}$ for straight steps and cutting steps at slow speed. These values for TP were 1. $07 \pm 0.03 \mathrm{Nm} / \mathrm{kg}$ and $-1.12 \pm 0.03 \mathrm{Nm} / \mathrm{kg}$, respectively. The mean moment at TP was greater than MS and WA, in both sidestep cutting and straight steps, as it was expected.

There was no statistically significant difference between DP moments at WA, MS, and TP phases of the stance periods during straight step and sidestep cutting at fast speeds (Table 5.4). At WA, the mean moment values were $0.51 \pm 0.03 \mathrm{Nm} / \mathrm{kg}$ and $0.51 \pm 0.02 \mathrm{Nm} / \mathrm{kg}$ for straight steps and cutting steps. At MS, those values were $-0.51 \pm 0.04 \mathrm{Nm} / \mathrm{kg}$ and $-0.58 \pm 0.05 \mathrm{Nm} / \mathrm{kg}$, respectively and for TP were $1.15 \pm 0.03 \mathrm{Nm} / \mathrm{kg}$ and $-1.11 \pm 0.02 \mathrm{Nm} / \mathrm{kg}$, respectively. The mean moment at TP was greater than MS and WA, in both sidestep cutting and straight steps. 


\subsubsection{Frontal plane kinetics}

The maximum eversion moments during sidestep cutting were higher than the corresponding straight steps with similar speeds (Fig. 5.8). The maximum eversion moments for the right and left steps in sidestep cutting occurred at approximately $25 \%$ of stance. The maximum eversion moment for right ankle during sidestep cutting at slow speed was $0.36 \pm 0.02 \mathrm{Nm} / \mathrm{kg}$ and for the fast speed was $0.45 \pm 0.02$ $\mathrm{Nm} / \mathrm{kg}$. These values were $0.29 \pm 0.02 \mathrm{Nm} / \mathrm{kg}$ and $0.38 \pm 0.02 \mathrm{Nm} / \mathrm{kg}$ for the left ankle, respectively.

During both sidestep cutting and straight steps at slow speeds, the IE moments were always in eversion direction (Table 5.1). There was a statistically significant increase in eversion moments at WA and MS during the sidestep cutting compared to straight walking. At WA, the mean moment values were $-0.04 \pm 0.01 \mathrm{Nm} / \mathrm{kg}$ and $0.11 \pm 0.02 \mathrm{Nm} / \mathrm{kg}$ for straight steps and cutting steps, respectively. At MS, those values were $-0.19 \pm 0.02 \mathrm{Nm} / \mathrm{kg}$ and $-0.29 \pm 0.03 \mathrm{Nm} / \mathrm{kg}$, respectively. The mean moments at TP in sidestep cutting and straight step were not statistically different. At TP, the mean moment values decreased slightly to $-0.16 \pm 0.02 \mathrm{Nm} / \mathrm{kg}$ and -0.15 $\pm 0.04 \mathrm{Nm} / \mathrm{kg}$ for sidestep cutting and straight step, respectively. The results of the comparison of the different stance phases during fast speeds were close to the results of the slow speed experiment. The difference in ankle moments between sidestep cutting and straight step at WA and MS and fast speed were statistically significant (Table 5.2). Also, the mean moments were close to slow speed results, for each experiment.

In the left step, the maximum average IE moment occurred at MS, in either of the straight step and sidestep cutting. There was a statistically significant difference between the IE moments in straight step and cutting step at both slow and fast speeds. At slow speed, the maximum average IE moment occurred in MS with values of $-0.14 \pm 0.02 \mathrm{Nm} / \mathrm{kg}$ for straight step and $-0.21 \pm 0.02 \mathrm{Nm} / \mathrm{kg}$ for cutting step (Table 5.3). At fast speed (table 5.4), these values were $-0.12 \pm 0.01 \mathrm{Nm} / \mathrm{kg}$ for 
straight step and $-0.22 \pm 0.02 \mathrm{Nm} / \mathrm{kg}$ for the cutting step, showing no significant change from slow speed results.

\subsubsection{Transverse plane kinetics}

The right ankle showed a large increase in external moments at the TP of the cutting steps (Fig. 5.9). EI moments were significantly different between straight walk and sidestep cutting for the right ankle although the average angles were statistically the same. The transverse plane moments during the WA and MS were small when compared to the sagittal and frontal plane moments and never became greater than $0.12 \mathrm{Nm} / \mathrm{kg}$. However, at TP during sidestep cutting, the external moment increased to a maximum of $0.38 \pm 0.02 \mathrm{Nm} / \mathrm{kg}$ and $0.44 \pm 0.03 \mathrm{Nm} / \mathrm{kg}$ for the slow and fast speed, respectively. Note that one of the challenges is that the hip joint also contributes to the transverse plane moments; and that the measured moments were the resultant moment generated by the hip and ankle. However, the measured transverse plane rotations were estimated as the relative movement of the foot with respect to the lower leg. Further investigation is required to quantify the contribution of the hip and ankle to the transverse plane moments during the pushoff.

All the moments were statistically significantly different when comparing the straight walk to sidestep cutting at all the phases of the steps in both slow and fast experiments. While the rotation moments for the cutting steps were generally greater than the straight steps at the corresponding speeds, they were significantly smaller than the sagittal and frontal plane moments at the corresponding stance phase. 

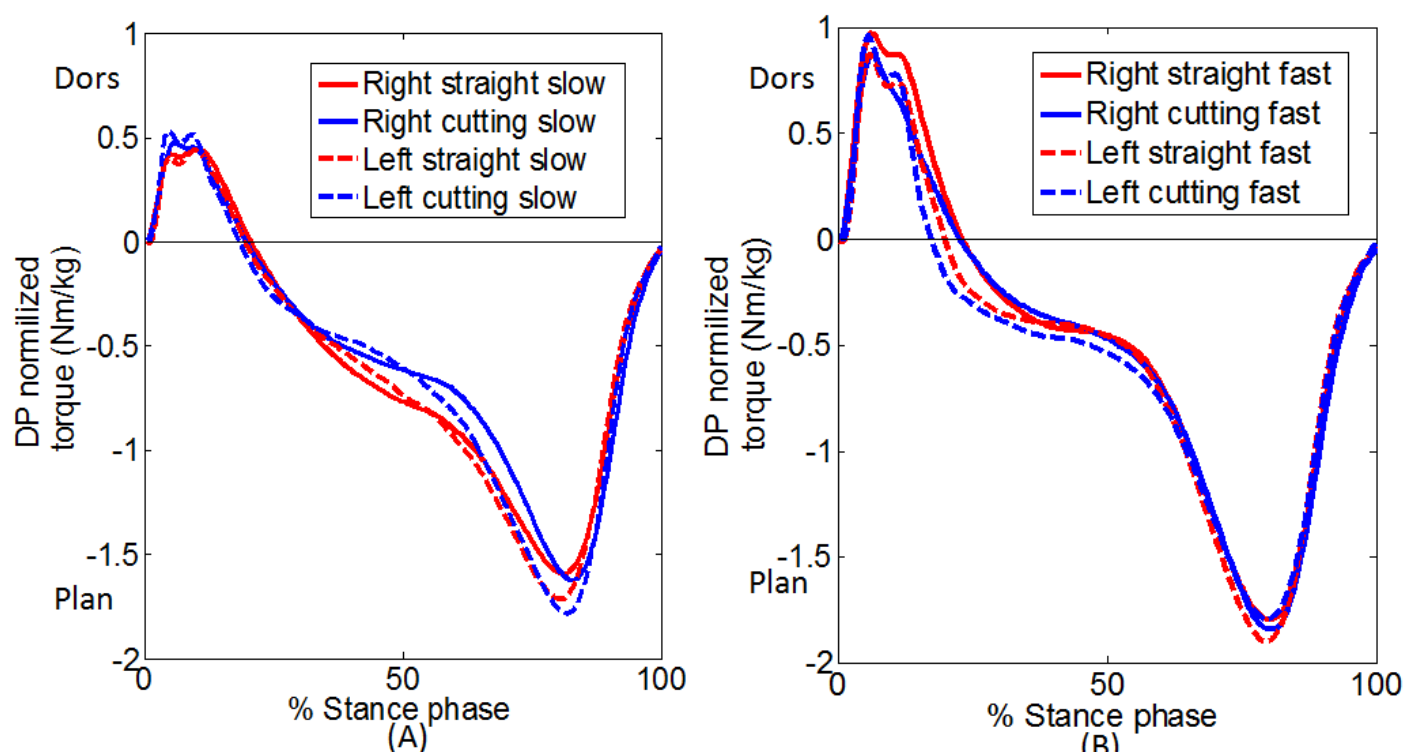

(B)

Figure 5.7. (A): Plot of the mean normalized ankle moments in DP at slow speed. (B): Plot of the mean normalized ankle moments in DP at fast speed. Positive moments are dorsiflexion, negative moments are plantarflexion.

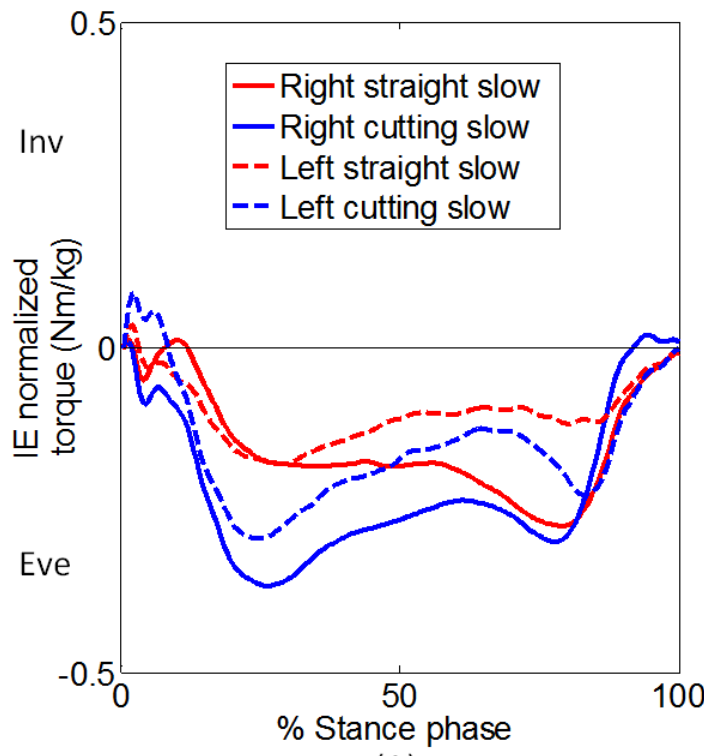

(A)

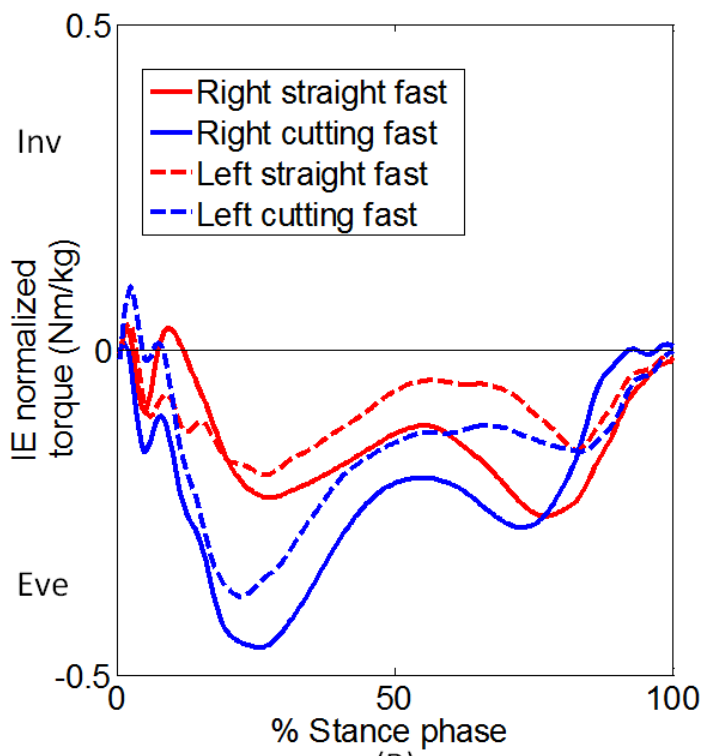

(B)

Figure 5.8. (A): Plot of the mean normalized ankle moments in IE at slow speed. (B): Plot of the mean normalized ankle moments in IE at fast speed. Positive moments are inversion, negative moments are eversion. 


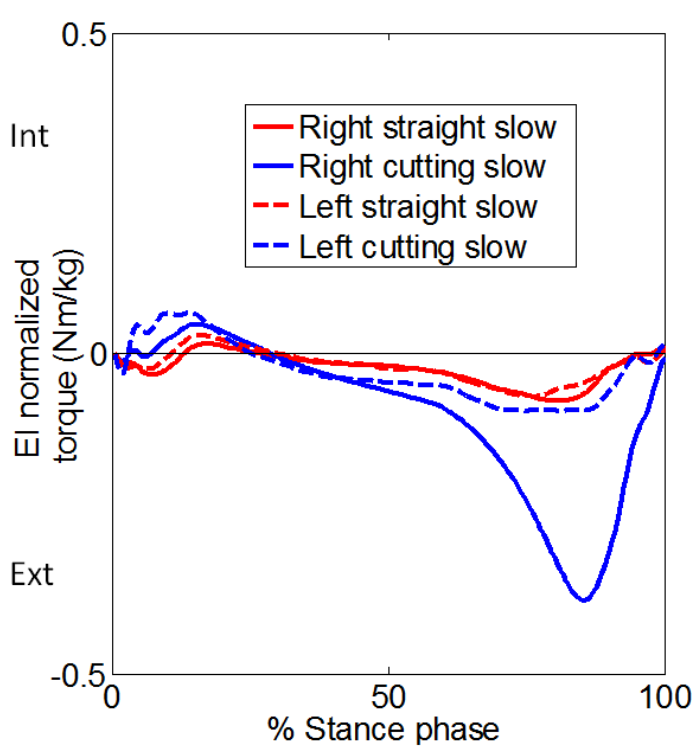

(A)

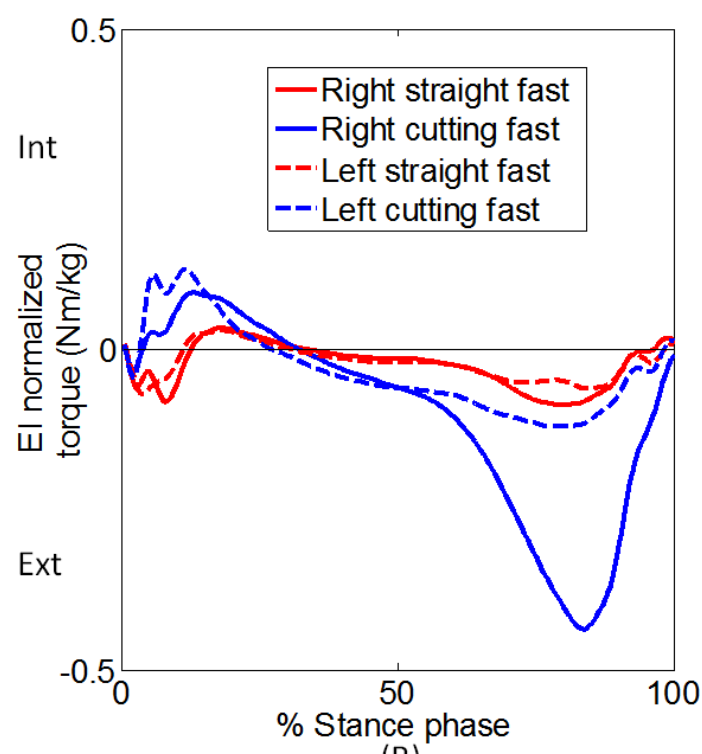

(B)

Figure 5.9. (A): Plot of the mean normalized ankle moments in EI at slow speed. (B): Plot of the mean normalized ankle moments in EI at fast speed. Positive moments are internal rotation, negative moments are external rotation.

\subsection{REMARKS ON THE ANKLE IMPEDANCE}

The ankle mechanics during sidestep cutting and straight walking at two different speeds showed that the ankle frontal plane rotation significantly changed during sidestep cutting when compared to straight walking. The data showed that during the right cutting step, increased eversion moment occurred at the WA and MS phases compared to straight walking. Similarly, an increased inversion was observed resulting from body leaning to the inside of the turn. While the body was leaning, the body weight generated a net moment to continually lean the body and increased the inversion angle, although the ankle torque was in eversion acting as a braking moment opposing the motion. At the end of the right cutting step the subjects immediately rotated and leaned their body to the right before the WA of the left foot. When the left foot contacted the ground it stopped the lateral motion of the body followed by the rotation of the body back to vertical position. This caused the left ankle to move from inversion back to a near neutral position without the need of increased eversion moments. 
Analysis of the ankle mechanics implied that the change of ankle impedance depends on the walking conditions. An impedance function maps the time history of ankle kinematics into the appropriate time history of ankle kinetics. If the change in ankle kinematics increases while there is no change or a decrease in ankle kinetics, the ankle impedance has decreased since more displacement was generated with the same or less moment. This was observed in frontal plane (i.e. IE motion) at the TP of the right ankle and at the WA of the left ankle at both speeds during sidestep cutting. At the TP of the right ankle at both speeds, the change in moment from straight to sidestepping cutting was near $0 \mathrm{Nm} / \mathrm{kg}$, while the change in angle was $14^{\circ}$. At the WA of the left angle at both speeds, the change in moment from straight to sidestepping turning was near $0 \mathrm{Nm} / \mathrm{kg}$, while the change in angle was $6^{\circ}$ at slow speed and $10^{\circ}$ at fast speed. At these phases, the ankle impedance decreased since the same amount of moment generated larger displacements. These results show that the ankle reduces its impedance in the frontal plane during turning to allow the body to lean.

On the other hand, if the ankle moments increase while the ankle rotations stay the same or decrease, the ankle impedance has increased since the increase in moment did not cause a proportional increase in displacement. This was observed in the transverse plane of the right step at both speeds, where the ankle rotations during cutting steps at both left and right side were statistically the same as the straight steps, but the amount of the ankle moments increased by $0.2 \mathrm{Nm} / \mathrm{kg}$ in the external direction. The TP of the right cutting step requires more investigation to identify the contributions of hip and ankle in the generated moments.

The results from the experiments described in this paper show the importance of frontal plane movement during turning. During straight walking, body weight shifts in the frontal plane from side to side in the frontal plane. During turning, the body leans to the inside of the turn in the frontal plane. It is hypothesized that this movement allows for a more natural gait when walking on incline planes perpendicular the direction of motion and also accommodates ankle motions 
required to walk on rough surfaces. The evidences obtained from the experiments described in this paper support the assertion that a 2-DOF ankle-foot prosthesis capable of impedance modulation in each axis could increase the agility during gait by mimicking true ankle mechanics.

\subsection{CONCLUSION}

In this section experiments were performed to measure ankle mechanics during straight walking and sidestep cutting at two different speeds. The study revealed no statistical difference in the sagittal plane ankle motion when comparing the sidestep cutting to straight walk. In the frontal plane, the right ankle showed progressively increasing inversion from $2^{\circ}$ up to $13^{\circ}$ while the left step, showed progressively decreasing inversion from $8^{\circ}$ up to $-4^{\circ}$ during the slow speed test. The changes in ankle kinematics in the frontal plane from sidestep cutting to straight walking were the most significant deviation of ankle kinematics, revealing its importance for shifting the body weight and change the walking direction during sidestep cutting. During both cutting and straight walking, the moments in the frontal plane were always in eversion. The observed eversion moments were more pronounced during the fast sidestep cutting with a normalized peak moment of $0.45 \pm 0.02 \mathrm{Nm} / \mathrm{kg}$. In the transverse plane, the least amount of moments occurred during walking. The frontal plane moments were significantly different when comparing straight walking to sidestep cutting; however, they were significantly smaller than the moments in the sagittal and frontal planes. The results indicate that an ankle-foot prosthesis capable of generating moments in both sagittal and frontal planes and with an impedance modulation similar to the human ankle will improve maneuverability and increase the agility for patients with limb loss. The necessity of a passive or active degree of freedom in the transverse plane needs further investigation to estimate the contribution of the hip joint to the generated moments measured at the foot-floor interface. 


\section{6- MULTI-AXIS CAPABILITY FOR POWERED ANKLE- FOOT PROSTHESES*}

Evandro M. Ficanha, Mohammad Rastgaar, Kenton R. Kaufman

\subsection{INTRODUCTION}

Recent advances in powered prostheses promise to significantly improve the quality of life and well-being for individuals with impaired mobility. A majority of people without disabilities, $61.4 \%$ to be exact, report their health to be excellent. In sharp contrast, only $28.4 \%$ of people with disabilities report the same. Moreover, people with disabilities are at a greater risk for secondary conditions (e.g., injury, obesity, and hypertension) that can further impact well-being and diminish overall quality of life (61). A better understanding of the complexities surrounding lower limb prostheses, which are needed for walking and daily activity will lead to increased health and well-being for the 1.7 million limb amputees in the US, the majority of whom have lower extremity amputations $(2,15)$. The ankle joint of lower extremity powered prostheses currently commercially available is capable of controlling only one degree of freedom (DOF) in the sagittal plane, allowing a focus on improved mobility in straight walking. Turning, however, plays a major role in

daily living activities and turning requires ankle torque in both sagittal and frontal planes. Therefore, the effectiveness of next-generation lower extremity prostheses may be significantly enhanced by improved understanding of ankle dynamics in both sagittal and frontal planes during different maneuvers and by implementing strategies to account for these intricacies in prosthesis design.

A multi-axis ankle-foot prosthetic robot capable of generating torques in both the sagittal and frontal planes with impedance modulation similar to the human ankle may improve maneuverability and increase mobility. It is shown that

* The material contained in this chapter is part of material previously published in the book: Neuro-Robotics: From Brain Machine Interfaces to Rehabilitation Robotics. The permission to use the material is shown in appendix $\mathrm{C}$. 
unilateral below-knee amputees who use passive prostheses rely more on their hip joint and expend $20-30 \%$ more metabolic energy compared to non-amputees at the same speed. As a result, their preferred speed of gait is $30-40 \%$ lower than nonamputees $(39,40)$ and their compensatory gait strategies results in asymmetrical gait patterns that affect joints in both lower limbs (41-44). Net positive work generated in the ankle contributes to propulsion in gait. It has been shown that a powered ankle-foot prosthesis reduces the metabolic costs of unilateral transtibial amputees during straight walking by providing sufficient power during push-off $(33,45)$. However, studies of four representative daily activities show that turning steps may account for an average of $25 \%$ of steps, ranging from $8 \%$ to $50 \%$ of all steps depending on the activity (1), which amputees accomplish using different gait strategies than non-amputees. While a non-amputee relies on the hip movement in the frontal plane and moment generated in the ankle joint, an amputee using a passive prosthesis relies on hip extension in the sagittal plane $(6,7,16,17)$. During a turn, modulation of ankle impedance in sagittal and frontal planes plays a major role in controlling lateral and propulsive ground reaction forces in order to accelerate the body center of mass along the gait path; thus, during a step turn, lateral and propulsive impulses are larger compared to a straight step (18). This difference will result in different gait strategies between amputees and nonamputees to compensate for the lack of propulsion in the passive prostheses to increase maneuverability (7). This suggests that an ankle-foot prosthesis controllable in two planes, i.e. dorsiflexion-plantarflexion (DP) and inversioneversion (IE) directions may increase the mobility by providing more assistance in turning. Additionally, the design of a feature that allows walking in arbitrary directions on slopes while conforming the foot to the ground profile and uneven surfaces may result in a more efficient gait.

Passive lower extremity prostheses do not store or generate energy. Understanding of the ankle's capability in impedance modulation and generating net positive work during the stance period of gait has influenced the design of new 
ankle-foot prostheses $(20-22,24)$. One design approach is based on storing energy during the heel strike and releasing it during the push-off before the trailing foot's heel strike. Collins and Kuo (23) developed a microprocessor-controlled artificial foot that limits the increase in metabolic cost to $14 \%$ compared to $23 \%$ that occurs with a passive prosthesis. The positive work by the prosthesis at the push-off partially compensates for the dissipative negative work at the heel strike of the trailing foot and lowers the redirection of the body's center of mass velocity (6266). On the other hand, there are powered prostheses capable of injecting energy to the system. Klute et al. developed a prototype using McKibben pneumatic actuators (67). Sup et al. developed a powered transfemoral prosthesis with active knee and ankle joints, each with one controllable DOF in the sagittal plane (25-27). The controller adjusts the impedance at a number of instants during gait by altering the neutral position of the ankle. Hitt et al. designed an ankle-foot prosthesis using a lightweight robotic tendon actuator that provided the majority of peak power for push-off $(68,69)$. Au et al. developed an ankle-foot prosthesis (29-31) that later transitioned into a commercially available ankle-foot prosthesis, BiOM (72). An adaptive muscle-reflex controller for this powered prosthesis was developed further by Eilenberg et al. using an ankle plantar-flexor model based on a Hill-type muscle and a positive force feedback reflex. A finite state machine determined the phase of the gait; hence, the appropriate ankle torques (70). The controller in the $\mathrm{BiOM}^{\circledR}$ allows for gait with different cadence over surfaces with different inclinations, e.g. uphill and downhill trajectories (8). Other commercially available powered transtibial prosthesis are the Proprio foot ${ }^{\circledR}$ from Össur and the Élan from Endolite; however neither provides a net positive work during the stance period $(9,10)$. $\mathrm{BiOM}{ }^{\circledR}$ provides the necessary energy during toe-off and generates a net positive work $(32,33)$ that has been shown to reduce the metabolic costs by $8.9 \%$ to $12.1 \%$ at different gait speeds compared to a passive prosthesis. The improvements occurred for gait speeds between $1 \mathrm{~m} / \mathrm{s}$ to $1.75 \mathrm{~m} / \mathrm{s}$; however, it did not change the metabolic cost at $0.75 \mathrm{~m} / \mathrm{s}$, suggesting a possible optimal range of speed for its 
design (34). The $\mathrm{BiOM} ®$ also increased the preferred gait speed by $23 \%$ (34) and lowered loading of the intact leg during level-ground walking, which may lower the risk of secondary complications (71).

Because the ankle is a biomechanically complex joint with multiple DOF, failure to incorporate full function of the ankle into prostheses design can inadvertently lead to secondary complications (72). Mechanical impedance of a dynamic system determines the evoked torque to the motion perturbations and is a function of the stiffness, visco-elasticity, and inertia of the system. Ankle mechanical impedance in no load-bearing conditions has been studied in the sagittal plane (73-87). Quasi-static ankle stiffness in both DP and IE were reported by Roy et al. (88), but coupling between these DOF was not assessed. The ankle impedance during load-bearing conditions has also been studied. In the frontal plane, Saripalli et al. (89) studied the variation of ankle stiffness under different load-bearing conditions. Zinder et al. (90) studied dynamic stabilization and ankle stiffness in IE by applying a sudden perturbation in the frontal plane during bipedal weight-bearing stance. In the sagittal plane, the quasi-stiffness of the ankle has been studied and suggests humans change their reflex ankle stiffness in response to unpredicted perturbations (91). Loram et al. determined that the intrinsic ankle stiffness during a quiet stance is almost constant with respect to ankle torque and suggested that the central nervous system contributes to the balance by modulating ankle stiffness especially with the triceps surae muscles in the sagittal plane $(92,93)$. Sasagawa et al. made a similar conclusion with subjects standing on inclined surfaces moving forward and backward (94). Ankle quasi-static stiffness was studied during quiet standing $(95,96)$ and locomotion $(20,22,47)$. Variations of ankle moment, ankle angle, and speed dependent hysteresis during gait cycles at different speeds was studied by Hansen et al (24). Also, dynamic stiffness of lower limbs during hopping and running was measured by Farley et al. $(24,97,98)$. Recently, Rouse et al. developed a perturbation platform to estimate ankle impedance during the foot-flat phase of the stance period of gait in the DP direction $(48,49,99)$. 
All of this prior work characterized the ankle's mechanical impedance in a single DOF and did not address the multidirectional characteristics of the ankle. The ankle is a biomechanically complex joint with multiple DOF where the major anatomical axes do not intersect and they are far from orthogonal, which could introduce a biomechanical coupling between DP and IE. Furthermore, single DOF ankle movements are rare in normal lower limb actions and the control of multiple ankle DOF presents unique challenges (46). Therefore, understanding the directional impedance of the ankle requires a multivariable identification approach. Multivariable mechanical impedance of the human ankle in both DP and IE directions in stationary conditions was estimated by Rastgaar et al. $(50,51)$ for dynamic mechanical impedance and Lee et al. (52-54) for quasi-static mechanical impedance. Ho et al. also studied the directional variation of quasi-static mechanical impedance of ankle (55-57). Further, Lee et al. developed a method for estimation of time-varying mechanical impedance of the ankle during the entire stride length for the subjects walking on a treadmill (58). Their study on ten unimpaired subjects showed consistent time-varying characteristics of ankle impedance during the entire stride in both sagittal and frontal planes.

In this chapter, we introduced the concept of a multi-axis powered ankle-foot prosthesis and showed the feasibility of this concept to the extent allowed by a proof of concept prototype. The design of the proof of concept prototype will be explained and the design kinematics and its mechanical impedance in non-load bearing conditions will be evaluated and discussed.

\section{2- MULTI-AXIS ANKLE-FOOT PROTOTYPE}

The result from the ankle rotations in three DOF suggested that a multi-axis mechanism in a prosthesis may enhance gait efficiency by adding control of ankle inversion/eversion during turning. This novel design is anticipated to enable the device to adapt to uneven and inclined ground surface condition and allow the 
amputees to benefit more from their prosthesis rather than using their hip joint; enabling a more natural gait with less stress in other joints.

Based on this concept, a prototype cable-driven ankle-foot prosthesis with two controllable degrees of freedom (Fig. 6.1) was designed to evaluate the feasibility of controlling a 2 DOF ankle joint. The design goals were to meet the ROM and angular velocity required for straight walk and step turn while providing sufficient torque for propulsion.

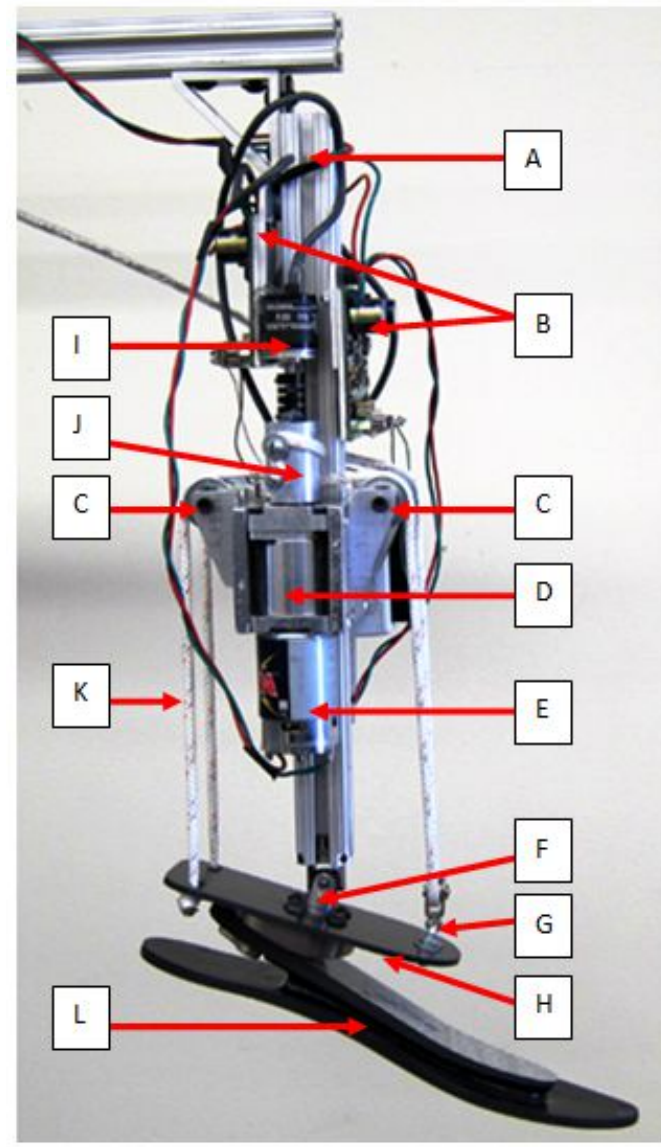

(A)

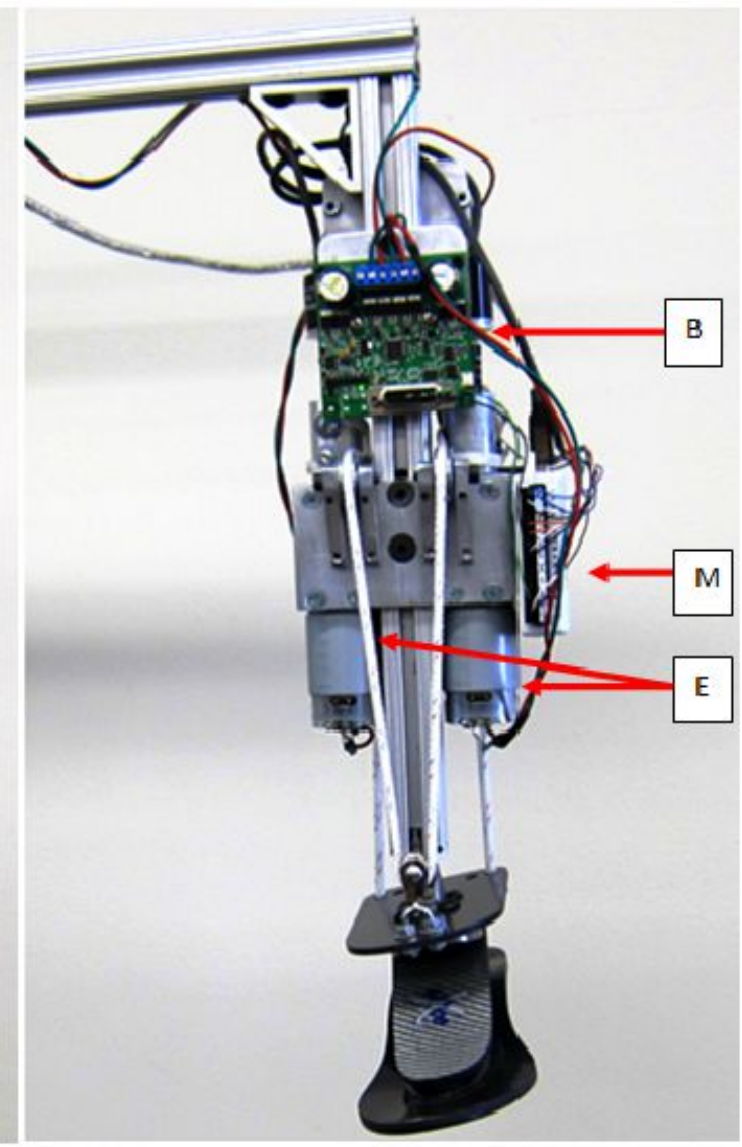

(B)

Figure 6.1. Prototype of a multi-axis powered ankle-foot prosthesis A- in plantarflexion and B-in inversion

The device consisted of a pylon (A), two DC motors (E) and planetary gearheads (D) that are powered by two motor controllers (B) that receive signals 
from a DAQ board (M) connected to a remote computer and two quadrature encoders (I). Two cable drums (J) transfer the required torque to the ankle through a nylon rope $(\mathrm{K})$ that passes through two pulleys $(\mathrm{C})$. The rope is looped around both the cable drums and secured to prevent slippage (Fig. 6.2). A universal joint (F) connects the pylon to the foot and an elastic carbon-fiber plate. The rope is attached to a carbon fiber plate $(\mathrm{H})$ that is connected to a commercially available prosthetic foot (Otto Bock Axtion $\left.{ }^{\circledR}\right)(\mathrm{L})$. In the aft side of the carbon fiber plate, the rope is mounted at both sides of the longitudinal axes of the foot. At the fore side, the rope passes through a pulley $(\mathrm{G})$ that is connected to the carbon fiber plate by a universal joint. The mechanism is capable of both dorsiflexion-plantarflexion when the motors rotate in opposite directions and inversion-eversion when the motors rotate in the same direction. Also, any combination of DP and IE can be achieved by combining different amounts of rotations at each motor.

The proposed design with two controllable DOF relies on the fact that three points are sufficient to define a plane in the space. As shown in Fig. 6.3, the three points $(\mathrm{A}, \mathrm{B}, \mathrm{C})$ can be used to define the rotations of the foot relative to the pylon about the $\mathrm{X}$ and $\mathrm{Y}$ axes that are equivalent to DP and IE, respectively. If the motors apply forces in the same directions, for example in the negative $\mathrm{Y}$ direction, points $\mathrm{A}$ and $\mathrm{B}$ will move downwards, while point $\mathrm{C}$ moves upwards, resulting in dorsiflexion. If the motors move in opposite directions, for example the right motor pull the rope in the positive $Y$ direction and the left motor pulls the rope in negative $\mathrm{Y}$ direction, point A moves upwards, while point $\mathrm{B}$ moves downwards generating foot eversion. At point $\mathrm{C}$, a pulley is mounted to the plate using a universal joint. Because point $\mathrm{C}$ is located on the axis of symmetry of the plate and the rope passes through the pulley at point $\mathrm{C}$, no DP motion is generated. The carbon fiber plate is a fundamental component of the design. It is reported that the average plantarflexion stiffness at 50\% maximum voluntary contraction (MVC) is $143 \mathrm{~N} . \mathrm{m} / \mathrm{deg}$ and is near constant from $30 \%$ to $80 \%$ MVC (87), thus the carbon fiber plate was design to have this stiffness in DP. The carbon fiber plate acts as a spring element in series 
with the cable and the foot. The cable needs to be always in tension to assure proper control over the foot and keep the carbon fiber plate under a bending moment. This assures the cable has a sufficient tension over the ROM of the foot providing that it can be controlled to mimic the mechanical impedance of a human ankle.

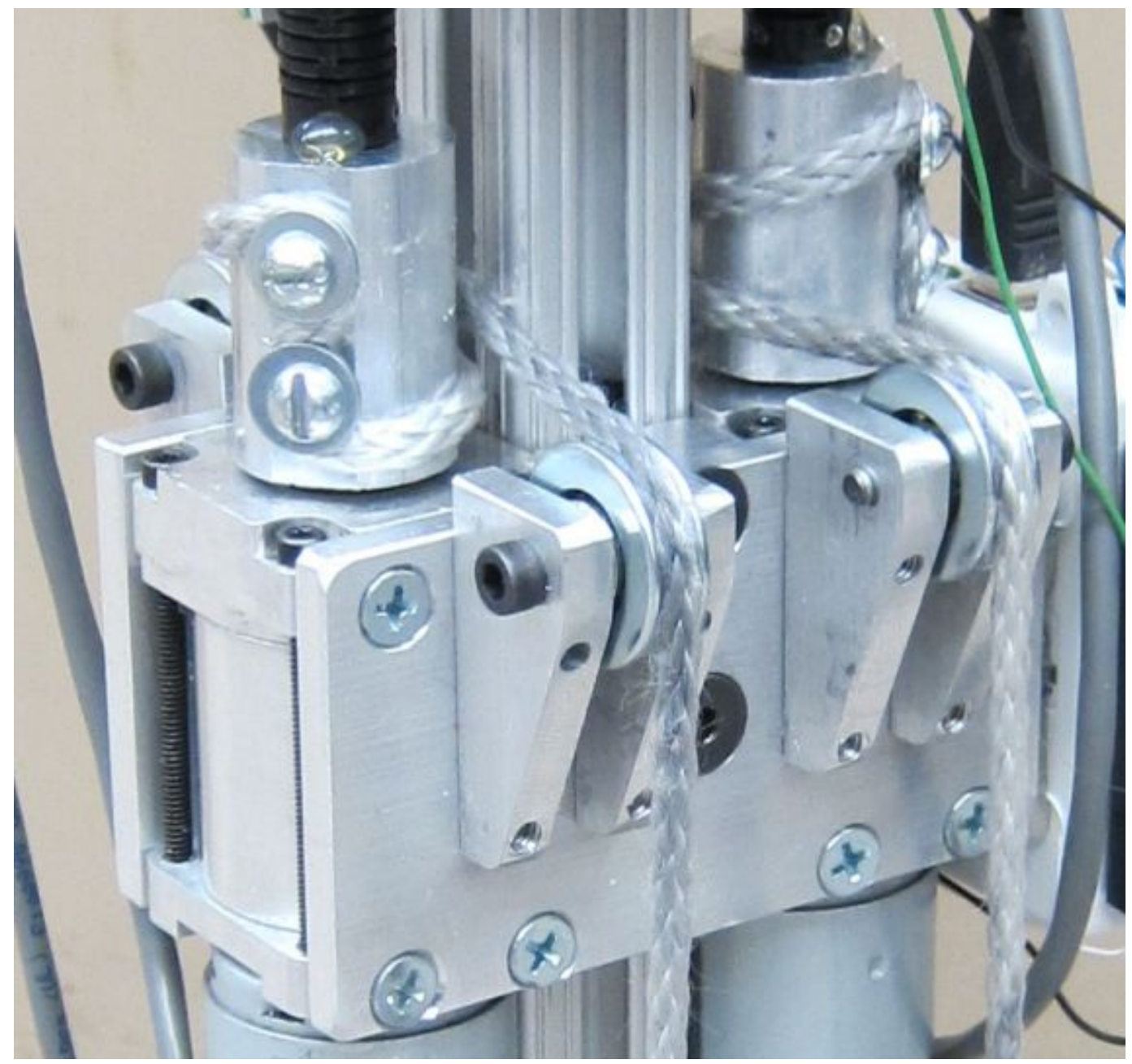

Figure 6.2. Cable drum connection to avoid cable slippage. 


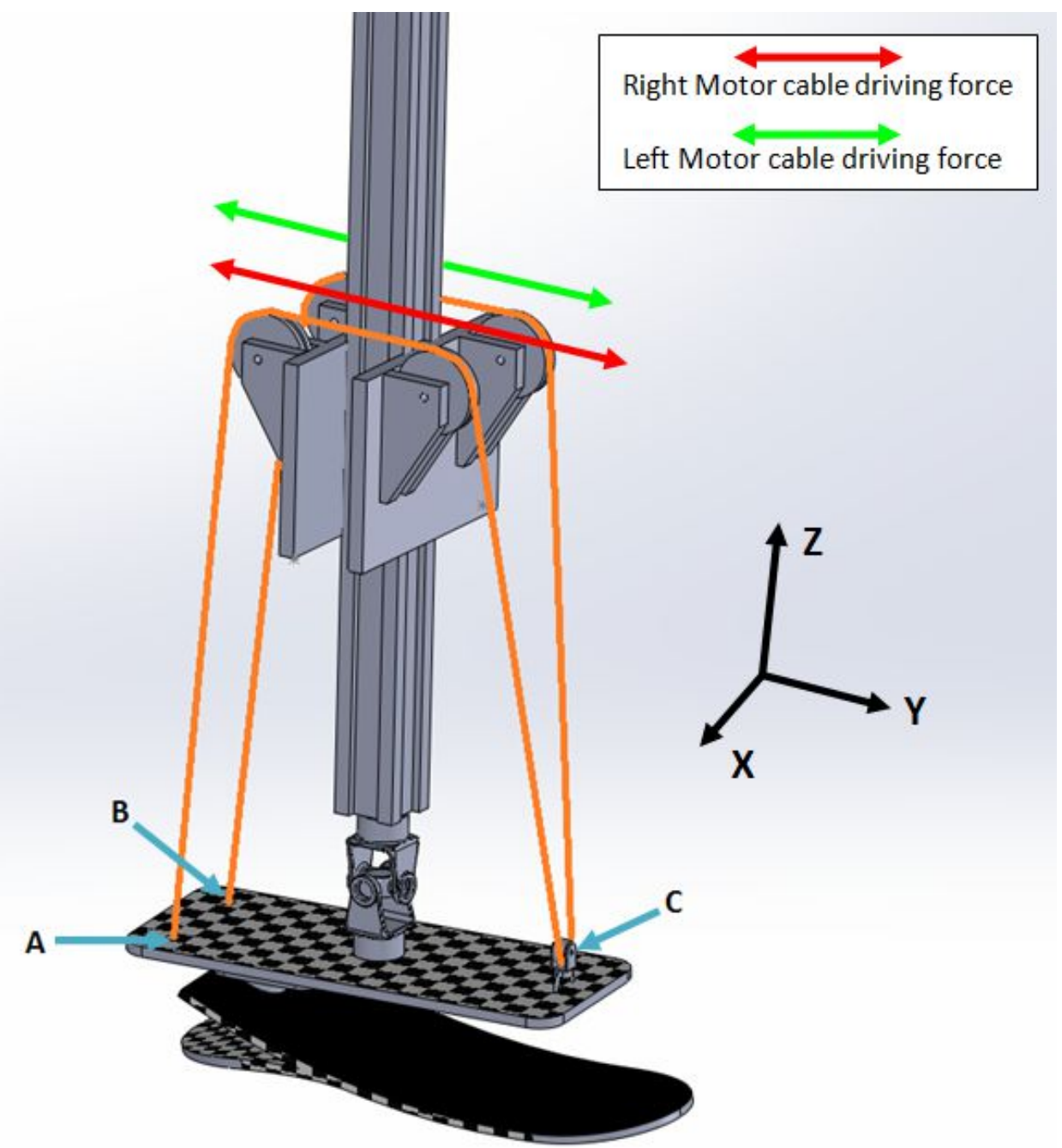

Figure 6.3. A simplified drawing of the cable driven mechanism showing three interaction points $\mathrm{A}, \mathrm{B}$, and $\mathrm{C}$ between the cable, carbon fiber plate, and motor drive forces

Providing sufficient power and torque at the ankle joint without significant increase in the weight of the powered prostheses is a challenging issue. For example, Hitt et al. used two parallel actuators to increase the power output for walking in increased speed (68). The steering mechanism proposed here also use two actuators; however, the design allows for generating a torque component in lateral plane. The cable driven design, besides the ability to control the ankle in two DOF, may provide a significant flexibility in managing the inertia of the ankle-foot 
prostheses too. A detailed description of the components used in this proof of concept prototype can be found in (19).

\subsection{EVALUATION OF THE DESIGN CONCEPT}

The developed prototype has been evaluated for meeting two criteria. First, the design kinematics should be capable of regenerating the same ankle rotation as the human ankle during the stance and swing phases of gait. Second, the multivariable impedance of the prototype ankle needs to be comparable to the mechanical impedance of human ankle.

\subsubsection{KINEMATIC EVALUATION}

Presently, two optical quadrature encoders (200 pulses per revolution) provide position feedback to a remote computer that uses a proportional plus rate controller to control the relative position of the foot with respect to the pylon. The controller uses a look-up table with recorded angles of the representative subject of the motion analysis experiment in both DP and IE. The input and output angles to the controller can be seen in Fig. 6.4 where the robot is moving at $50 \%$ of the walking speed. For ease of comparison, the output plots have a time shift to remove the 80 milliseconds delay of the output. Also, all signals are filtered with a low-pass filter with a cutoff frequency of $5 \mathrm{~Hz}$ to remove sensor noise from the output signal. The current prototype was developed as a proof of concept to validate the design kinematics; therefore, faster motors and sensors with lower noise levels will be used in future designs. 

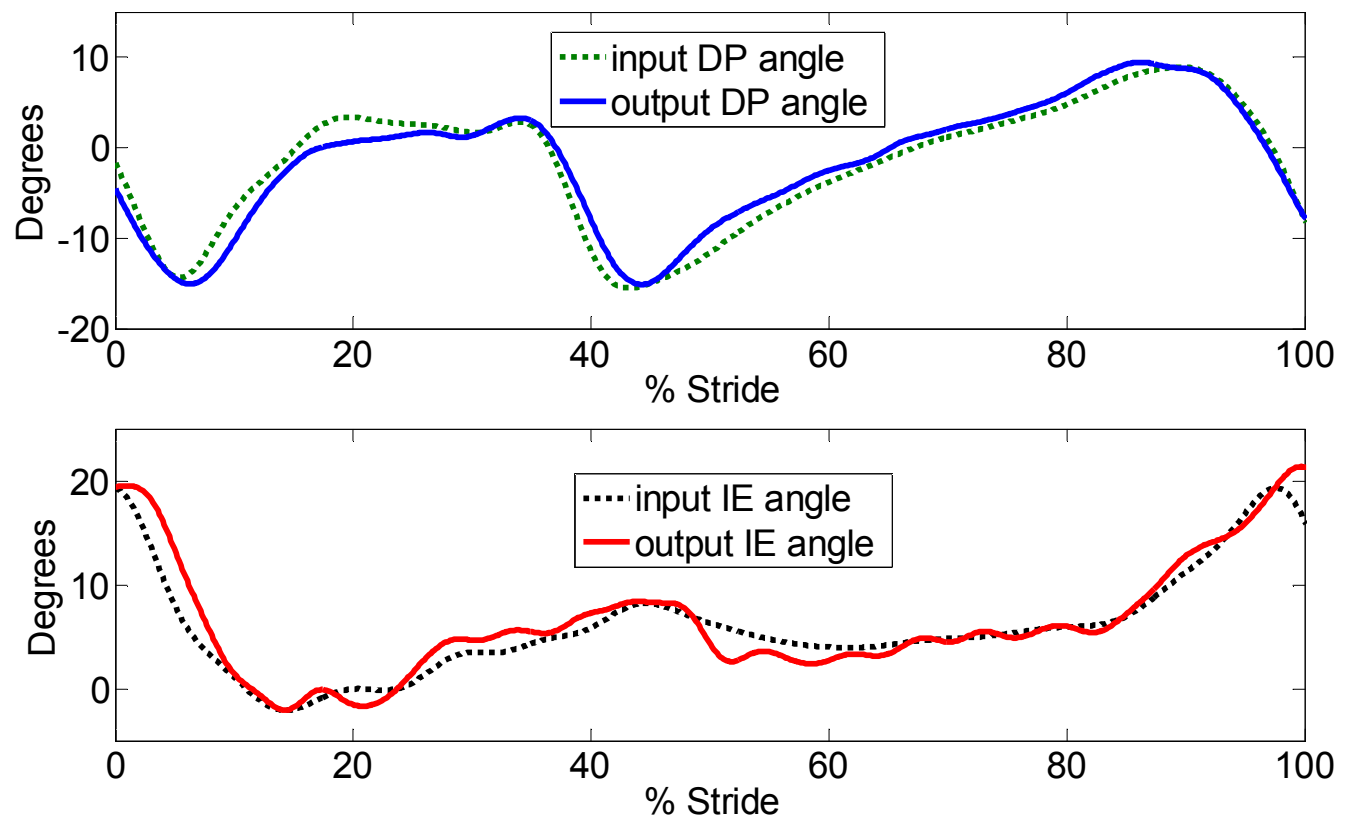

Figure 6.4. Input and compensated output for time delay (80 milliseconds) of the ankle-foot prosthesis during swing and stance phases of a step turn at $50 \%$ of the walking speed with no load

\subsubsection{MECHANICAL IMPEDANCE ESTIMATION}

Recently, hierarchical control strategies have been developed for impedance control of active prostheses $(26,70)$. The higher level control identifies the gait cycle, and the lower level control regulates the actuators for a proper impedance characteristics. Following the same strategies, an ankle-foot prosthesis can be designed to have an initial mechanical impedance similar to a human's ankle. This may provide a faster modulation of the ankle impedance based on the state of the gait cycle.

The purpose of this evaluation was to identify the passive impedance of the prosthetic device and compare it to the impedance of human subjects. The impedance estimation experiment setup was similar to the procedure reported in $(50$, 51), where an Anklebot ${ }^{\circledR}$ was used to apply random torque perturbations to the ankle joint in DP and IE directions with a bandwidth of $100 \mathrm{~Hz}$ and record the provoked ankle angles. A stochastic system identification method was used to estimate the 
multivariable mechanical impedance of the ankle using the collected data. Similar procedures were used for estimation of passive mechanical impedance of the anklefoot prosthesis while all its controllers were turned off. The experimental setup can be seen in Fig. 6.5, where the two devices were attached mechanically to each other. The Otto Bock Axtion ${ }^{\circledR}$ foot and its rubber foot shell were inserted in the same type of shoe used in human tests to ensure consistency in the experiments. Similarly, the same test was done on a representative human subject for comparison with the prosthesis. Impedance test of the human subject was performed with both relaxed muscles and $10 \% \mathrm{MVC}$ of the tibialis anterior following the procedures described in (51). The EMG signals were monitored using a Delsys Trigno Wireless EMG System $^{\circledR}$ with surface electrodes placed at the belly of the tibialis anterior. The EMG signal was sampled at $2 \mathrm{kHz}$ and the root-mean-square value of a window of $13.5 \mathrm{~ms}$ of data calculated and displayed on a computer screen in order to provide a visual feedback to the participant for maintaining constant muscle activity. The results can be seen in Fig. 6.6 and 6.7.

Figure 6.6 shows the Bode plots of the prototype prosthesis, human subject's ankle with relaxed muscles, and human subject's ankle with 10\% MVC impedances in DP direction. The quasi-static stiffness of the prototype prosthesis, which is the impedance magnitude at low frequencies, was $39.5 \mathrm{~dB}(94 \mathrm{~N} . \mathrm{m} / \mathrm{deg})$ in DP at $1 \mathrm{HZ}$. Also, it shows a relatively linear impedance and phase over the frequency range of interest (0 to $5 \mathrm{HZ})$. The human subject showed similar stiffness in DP for the cocontraction testes and lower stiffness in passive test when compared to the prosthesis. IE impedance magnitude (Fig. 6.7) of the prosthesis was between the active and passive stiffness of the human sample with a value of $24.24 \mathrm{~dB}$ (16 N.m/deg) at $1 \mathrm{~Hz}$. 


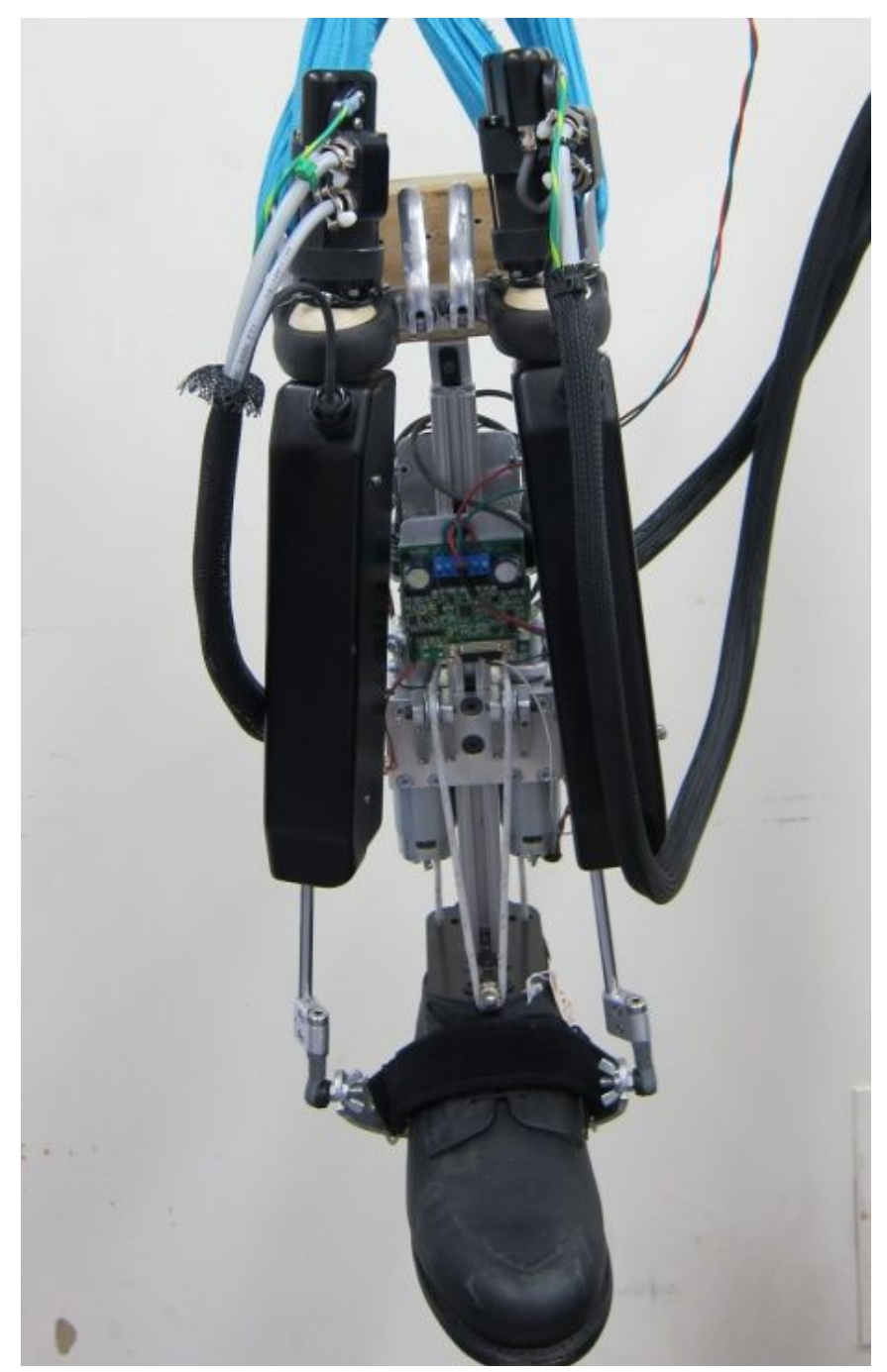

Figure 6.5. Anklebot attached to the prosthetic foot to measure the mechanical impedance of the ankle-foot prosthesis

The maximum lifting force of the robot in the $\mathrm{z}$ axis was performed. As expected, the carbon fiber plate flexed due to the applied torque. Since the encoders read the cable displacement instead of foot angles, the controller perceived the deflection of the carbon fiber plate as an angular displacement of the foot. This caused the position controller to reduce the torque being applied prematurely, and thus the maximum lifting force was less than anticipated, although it was still powerful enough to lift a $72 \mathrm{KG}$ person. Future designs will benefit from position 
sensors which can measure the foot angles directly to increase the precision of the position and the resulting torque.
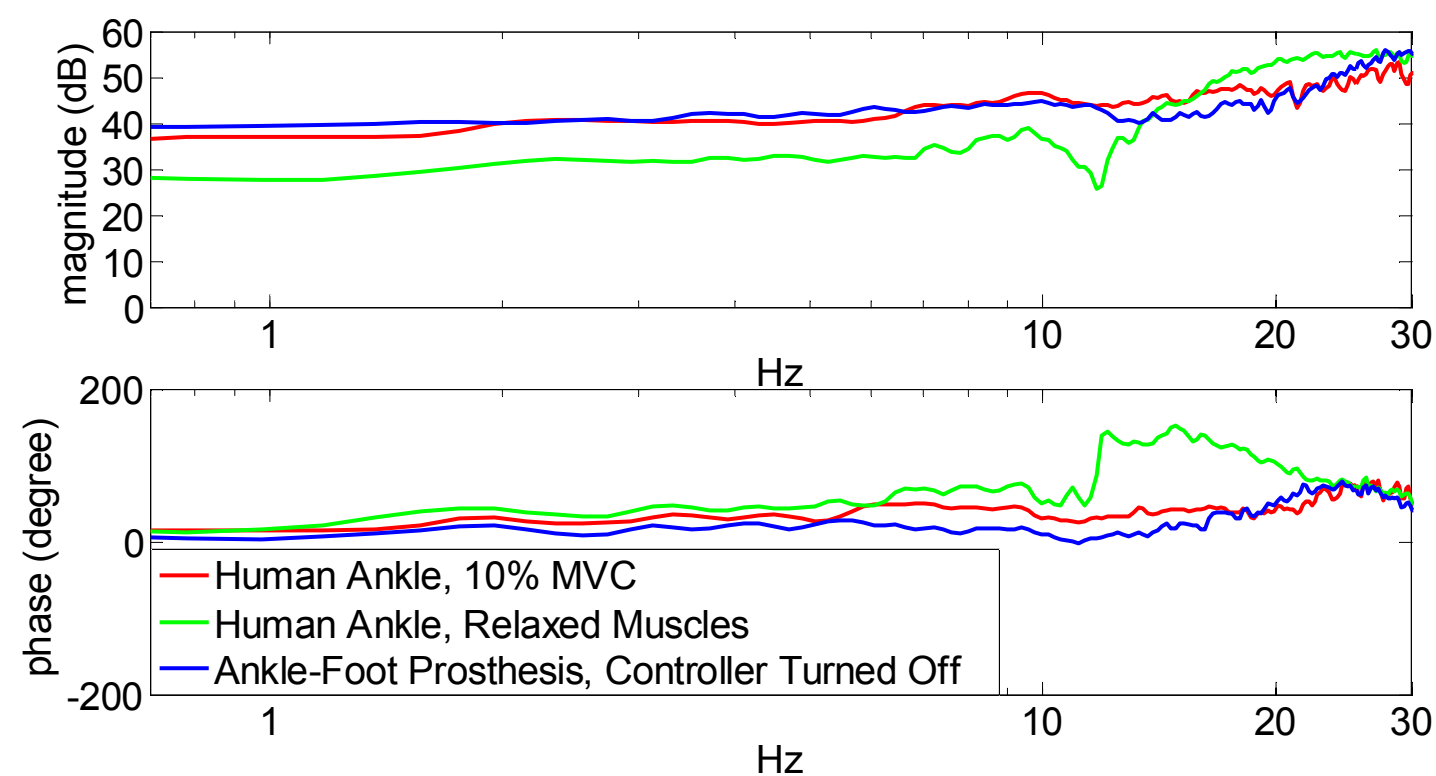

Figure 6.6. Plots of the magnitude and phase of the impedance in DP rotation of the prosthetic robot and a human subject with relaxed muscles and $10 \%$ cocontraction.

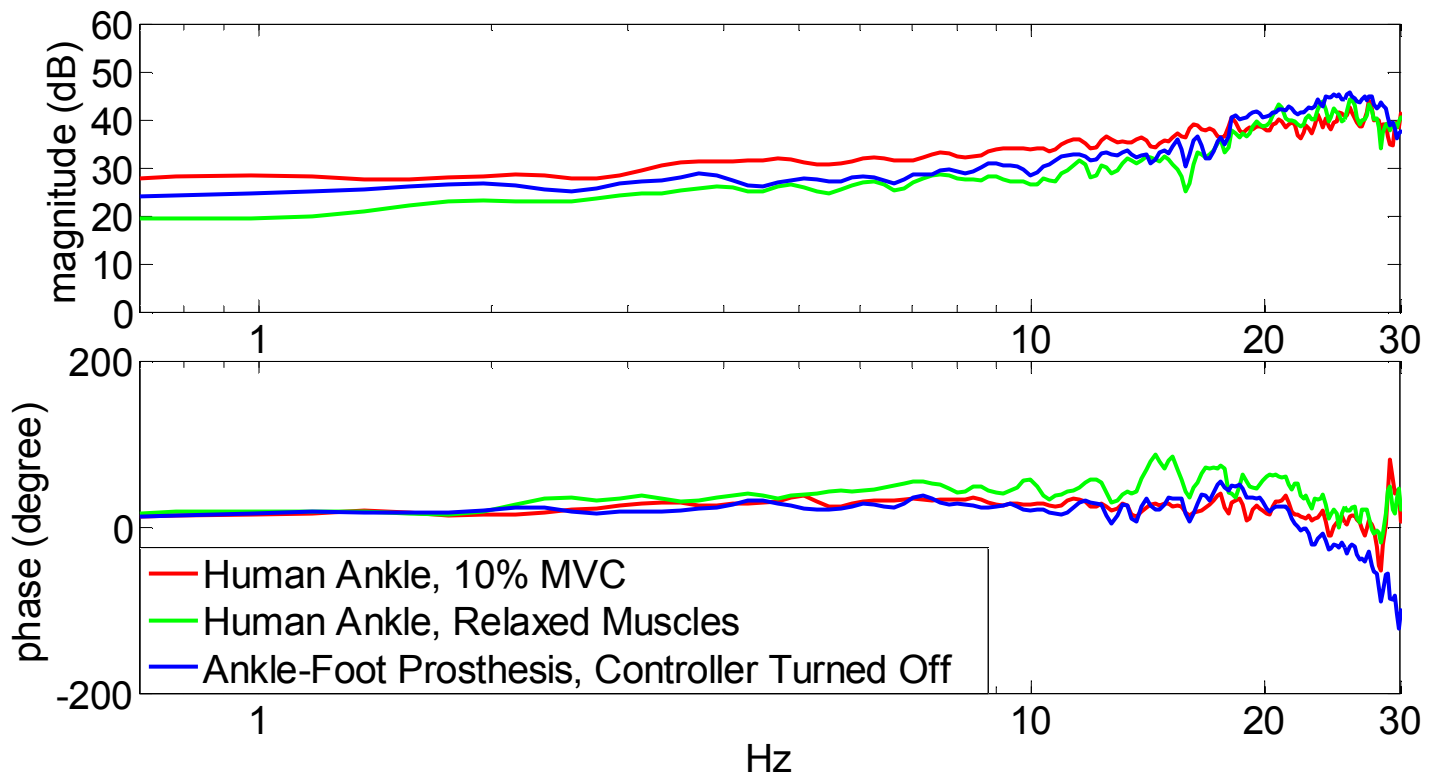

Figure 6.7. Plots of the magnitude and phase of the impedance in IE rotation of the prosthetic robot and a human sample with relaxed muscles and $10 \%$ cocontraction 
Testing the range of motion in IE, it was found that the IE motion might become unstable when in excess of 62 degrees due to an external force. This is the equivalent of rolling the ankle, which is a common injury among active people and are mostly in inversion (89). From the gait analysis experiment, it was seen that the maximum rotation in IE (table 6.1 ) was $19.72^{\circ}$, and thus instability should not impose a significant issue during normal gait.

The current developments in prosthesis suggests that the control of the ankle joint in prosthetic ankle-foot devices will mimic the time-varying impedance of the ankle in two DOF during different phases of stance period in different gait scenarios while providing the required torque. Recent work by Lee et al. and Rouse et al. (49, 58) are notable for estimating the time-varying ankle impedance during both swing and stance periods of gait along a straight path. To estimate the time-varying impedance of the ankle during a turning maneuver, a perturbing walkway may be necessary. The implemented ankle-foot mechanical design, although in early stages of development, showed anthropomorphic characteristics. The design was successful at mimicking human motion, and showed mechanical impedance similar to the human ankle. 


\section{7- GAIT EMULATOR FOR EVALUATION OF ANKLE- FOOT PROSTHESES CAPABLE OF TURNING*}

Evandro Ficanha, Mohammad Rastgaar, Kenton R. Kaufman

\subsection{BACKGROUND}

One of the challenges in the development of prostheses is the testing and tuning of the mechanism before testing with human subjects. It is a common practice in the industry to use testing platforms to tests new products for different properties such as strength and fatigue; however, few platforms have been developed for testing and tuning of ankle-foot prosthesis. Before testing the lower extremity prostheses with human subjects, the designers need to assure that the device would perform as designed, otherwise it may result in injury. Another issue that may arise is the lack of repeatability during evaluation experiments with human subjects. Humans have a remarkable ability to adapt to new environments; hence studying the effects of any tuning in the prosthesis performance may not be conclusive, as it may not always be clear if the outcomes are due to the changes in the prosthesis, or due to the adaptation by the amputees.

Richter et al. reported a testing apparatus to evaluate the lower extremity prostheses in the sagittal plane (100). Sagittal plane testing of leg-prostheses meets the requirements for testing the currently available powered ankle-foot prostheses, which control the ankle only in the sagittal plane and focus on straight walking. However, depending on the activity, turning steps may account for up to $50 \%$ of the steps (1). Turning steps require modulation of the torques and angular displacements of the ankle-foot mechanisms in both the sagittal and frontal planes resulting in increased lateral and propulsive impulses when compared to straight walking (18).

*The material contained in this chapter was previously published in the ASME Journal of Medical Devices. The permission to use the material is shown in appendix D. 
The need of a device to help in testing and development of ankle-foot prostheses with two DOF (degrees of freedom) motivated the present work. A gait emulator that can be used with both standard and circular treadmills was developed (Fig. 7.1), allowing testing and tuning of different types of ankle-foot prosthesis, including active and passive, and working as an intermediate step between design and human trials. The gait emulator was used together with a custom-made circular treadmill to develop and tune an ankle-foot prosthesis with two DOF in the frontal and sagittal planes.

\subsection{METHODS}

The gait emulator (Fig. 7.1 and 7.2) is designed to work with both the circular treadmill as well as regular gym treadmills. In Figs 7.1 and 7.2, the gait emulator is presented with a circular treadmill and a passive prosthesis (A). Unlike a regular treadmill, where the user walks in a straight line, in a circular treadmill the user needs to always be turning to stay on top of the treadmill; which makes it suitable for testing and tuning ankle-foot prosthesis for turning. The circular treadmill is composed of a wooden disk with a $1 \mathrm{~m}$ radius (B). On the outside lower edge of the disk, eight coaster wheels (C) are connected for weight bearing. Also, a heavy-duty turn table (not visible) is mounted in the center of the disk for both weight-bearing and constraining the disk from sliding on the horizontal plane. A $343 \mathrm{~W}$ brushed motor (Banebots First CIM) and planetary gearhead with a 64:1 gear ratio (D) provide power for the rotation of the disk using a belt system $(\mathrm{J})$ with a reduction ratio of 5.3:1 resulting in a total 339:1 reduction from the DC motor to the circular treadmill. This results in a maximum walking speed of $1.63 \mathrm{~m} / \mathrm{s}$ which is sufficient considering the average preferred human walking speeds for young adults is $1.30 \pm$ $0.1 \mathrm{~m} / \mathrm{s}$ (101). The speed of the treadmill disk is controlled using an open loop speed controller and powered by a motor controller (K) (RoboteQ LDC 2250C) and the power is supplied by a $12 \mathrm{~V}$ deep cycle battery $(\mathrm{L})$. 


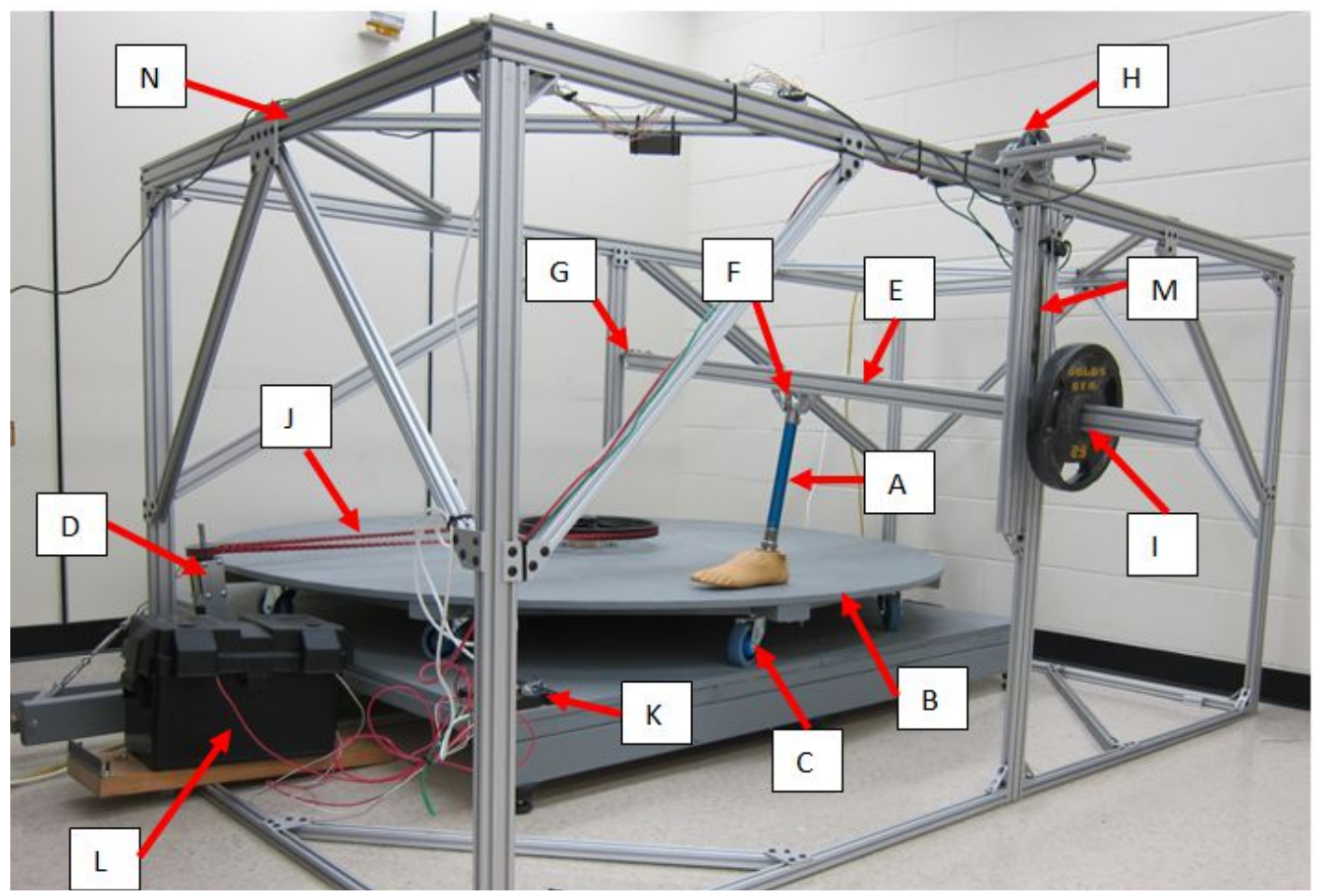

Figure 7.1: Gait emulator and circular treadmill and its main components.

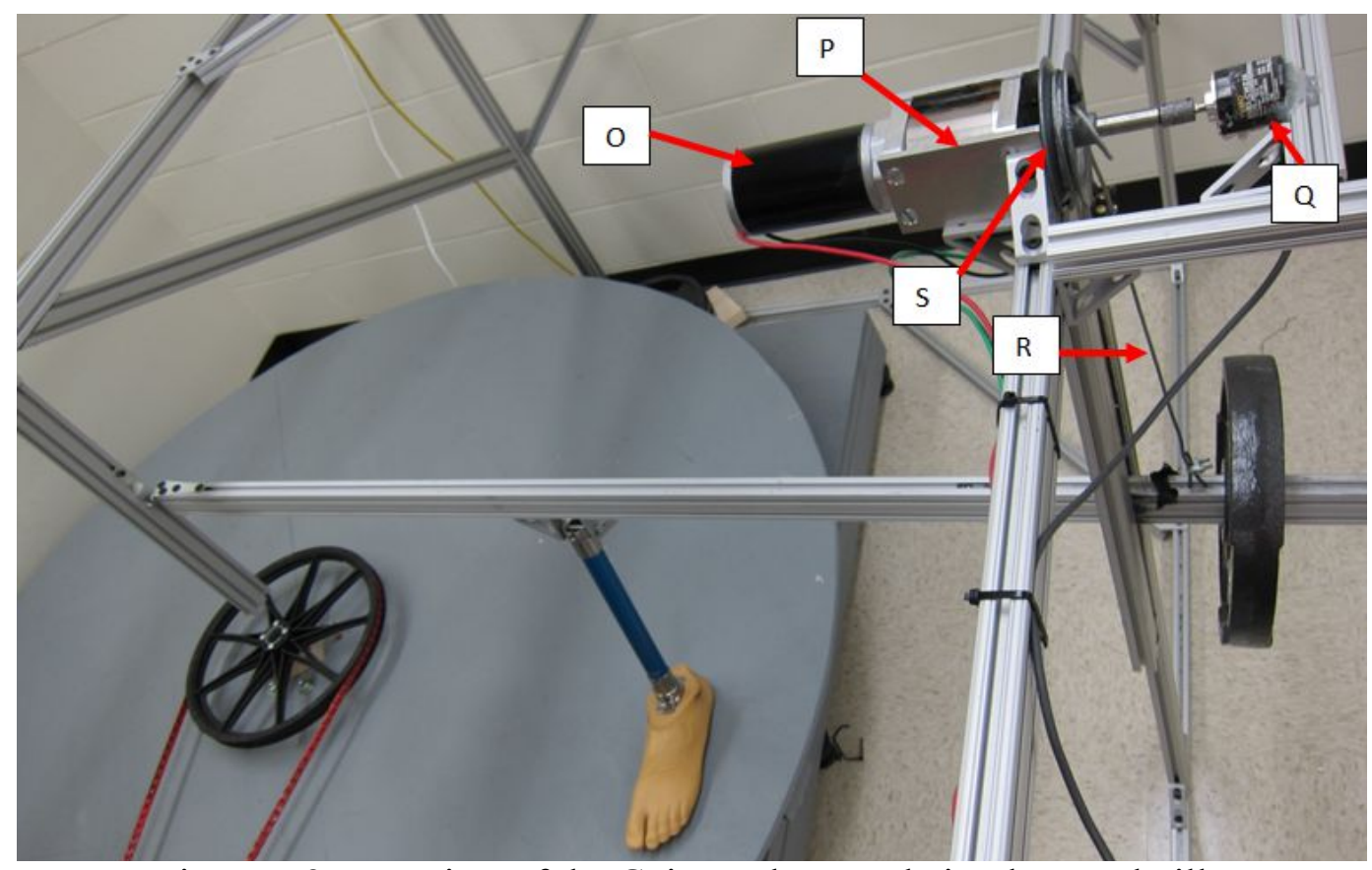

Figure 7.2: Top view of the Gait emulator and circular treadmill. 
The prosthetic ankle-foot $(\mathrm{A})$ is connected to a horizontal bar $(\mathrm{E})$ by a universal joint (F), which acts as a passive knee. At one end, the bar (E) is connected to a pivot $(\mathrm{G})$ and at the other end to a cable $(\mathrm{R})$. The cable itself is connected to a $343 \mathrm{~W}$ brushed motor (O) (Banebots First CIM) and gear box with a 36:1 gear ratio (P). A cable drum with $10 \mathrm{~cm}$ diameter $(\mathrm{S})$ is connected to the gearbox $(\mathrm{P})$, which by winding the cable $(\mathrm{R})$ can raise and lower the bar and the prosthesis. The motors for the lifting mechanism and the treadmill are controlled by the same motor controller $(\mathrm{K})$, which is capable of controlling two DC motors. The bar (E) is also capable of bearing loads (I) that is supported by the prosthetic leg when the weight is lowered during the stance or by the lifting mechanism when the leg is raised during the swing. The bar is constrained to only slide up and down using a sliding mechanism (M). The amount of weight can be modified to simulate different users' weight. The prosthetic leg, bar, motor and gear box, and weights are attached to an aluminum frame $(\mathrm{N})$ that is not coupled to the treadmill except through the foot at the time of the stance when it contacts the wooden disk. The circular treadmill can be easily replaced by a standard gym treadmill as it is not directly connected to the gait emulator.

The lifting mechanism uses a PD controller (Fig. 7.3) with feedback from a quadrature encoder (Q). The PD controller input is a sine wave with the same frequency as the gait. The sine wave has an amplitude of $\boldsymbol{A}$ degrees, which corresponds to the cable drum rotation, frequency of steps $(\omega)$, and phase shift $\varnothing$ to synchronize the gait emulator to the motion of powered ankle-foot prostheses. These values are dependent on the prosthetic ankle-foot tuning, amount of weight being used, and the position of the prosthesis with respect to the frame and treadmill. The lifting mechanism is capable of lifting $118 \mathrm{~kg}$ at $10.6 \mathrm{~m} / \mathrm{s}$. However, the weight supported by the prosthetic leg is higher due to leverage, resulting on the weight supported by the prosthesis to be a function of the position of the pylon with respect to the beam (E) and the amount of weight installed on the mechanism. Using the presented circular treadmill, which was designed to emulate gait during turning, the 
radius of the turn of each step can be increased or decreased by sliding the frame (N) so the foot is closer to or farther away from the center of the treadmill. Currently, the prosthesis pylon angle is not controlled, and the "knee" joint is a passive one DOF joint resulting in a free swing forward phase. The versatile design of the gait emulator allows to use active knees to control the swing phase speed or to be modified to be used to test and tune prosthetic legs containing both hip and knee with either passive or active joints.

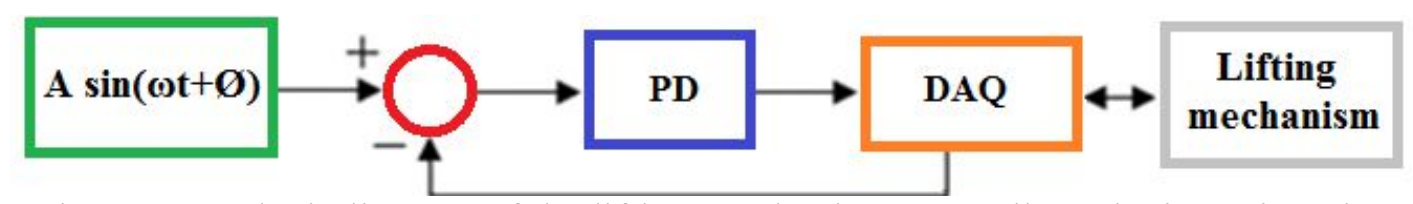

Figure 7.3: Block diagram of the lifting mechanism controller. The input is a sine wave with amplitude of A degrees of the cable drum rotation, frequency of steps $\omega$, and phase shift $\varnothing$ to synchronize the gait emulator to the motion of the ankle-foot prostheses.

\subsection{RESULTS}

The gait emulator and circular treadmill were successful at mimicking gait during turning using both passive and active prostheses. Fig. 7.4 shows a prototype ankle-foot prosthesis with 2 controllable DOF in both the frontal and sagittal planes at different states of the gait while walking on the circular treadmill using the gait emulator with a $25 \mathrm{~kg}$ load. The input sine wave was set with amplitude of the rotations of the cable drum to $100^{\circ}$ which corresponds to a vertical displacement of $8.9 \mathrm{~cm}$ of the knee joint, and the gait frequency was set to 48 steps per minute. The active ankle-foot prosthesis shown in Fig. 7.3 uses two PD controllers to control dorsiflexion-plantarflexion and inversion-eversion rotations. The gait emulator was used to tune the prosthesis PD controllers, which used pre-recorded trajectories of the human ankle to adjust the neutral position of the ankle and position feedback from the prosthesis' quadrature encoders to estimate the appropriate motor inputs. 


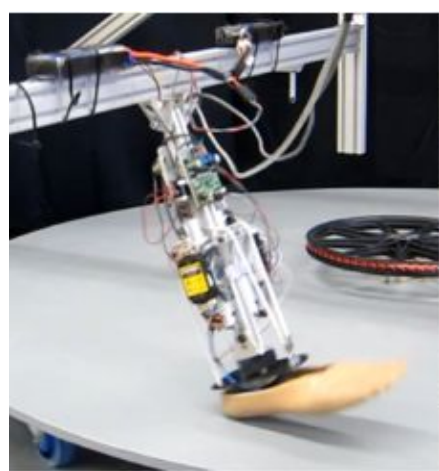

(A)

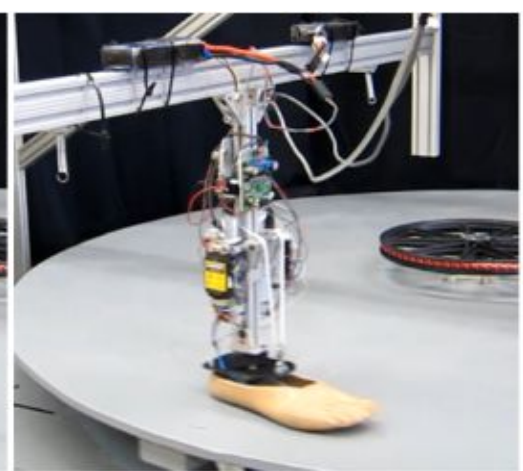

(B)

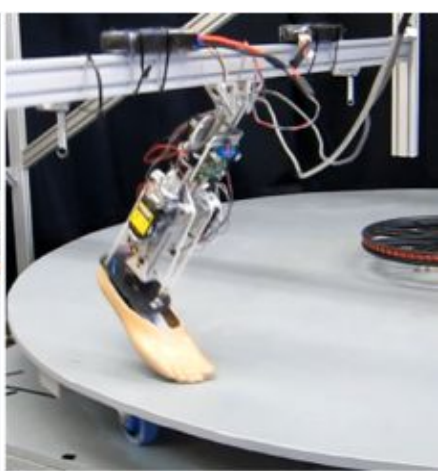

(C)

Figure 7.4: Gait emulator and a prosthetic ankle-foot robot with two controllable DOF in both the frontal and sagittal planes at different states of the gait. A- Heelstrike, B- Foot-Flat, and C- Push-off.

\subsection{INTERPRETATION}

The gait emulator and treadmill provided a platform for testing ankle-foot prostheses, which allows for consistent and repeatable measurements and tuning. In the presented work, the gait emulator and circular treadmill were used to tune the PD controllers of a powered ankle-foot prosthesis as shown in Fig. 7.4. Future research will include a motion capture camera system to measure the kinematics of the prosthesis during the gait. Also, the camera system will be used to measure the human gait while walking on the treadmill for comparison. The treadmill will also have modular terrain profile elements, which can be added to simulate off-camber turn, ascending, descending, and traversing the slopes. These terrain profile elements will be used to tune the prostheses' controller for disturbance rejection and surface profile adaptation. 


\section{8- IMPEDANCE AND ADMITTANCE CONTROLLER FOR A MULTI-AXIS POWERED ANKLE-FOOT PROSTHESIS*}

Evandro M. Ficanha, Mohammad Rastgaar

\subsection{ABSTRACT}

This paper introduces a finite state machine to select between impedance and admittance control for a powered ankle-foot prosthesis controllable in both Dorsiflexion-Plantarflexion (DP) and Inversion-Eversion (IE). Strain gauges are installed on the prosthesis' foot to measure the strain caused by ground reaction forces, which are correlated to the external torques in DP and IE. The external torques are used for the admittance and impedance controllers. Additionally, the finite state machine uses the strain gauges feedback to detect the heel-strike and switch to admittance control. The admittance control accepts torque feedback to generate motion, this way larger feedback torques effectively reduces the stiffness of the ankle. During push off, the finite state machine switches to impedance control, accepting motion feedback to generate the appropriated torques. The quasi-static stiffness of the prosthesis with impedance control was tested, showing a near linear relationship between the torque feedback gain and the stiffness of the ankle. The finite state machine and controllers were also evaluated using a custom-made circular treadmill and the results were compared to the results of position and passive controllers; showing that the impedanceladmittance controller was capable of tracking the desired input trajectory while decreasing the required torque at the ankle joint.

*The material contained in this chapter is part of material previously published in the 2014 ASME Dynamic Systems and Control Conference. The permission to use the material is shown in Appendix D. 


\subsection{INTRODUCTION}

Walking in a straight line requires a complex modulation of muscle contractions to control the ankle's stiffness and generate forward propulsion. Similar muscle contractions are required to generate the appropriate ground reaction forces to steer the body while turning (7). Below knee amputees with passive prosthesis expend $20-30 \%$ more energy than non-amputees to walk at the same speed, resulting in a preferred walking speed which is $30-40 \%$ slower than non amputees $(39,40)$. As a possible solution, powered prostheses have been developed and it is shown that they reduce the metabolic cost during straight walking by providing energy to the gait at push-off $(33,45)$. While the focus on developing powered prostheses has been on increased mobility in forward locomotion; it has been shown that daily activities contain an average of $25 \%$ turning steps (1). Turning requires modulation of the ankles impedance in both DP and IE directions to control the lateral and forward reaction forces to maintain the body's center of mass along the desired trajectory; resulting in increased lateral and forward forces when compared to the straight walking (18). Due to the lack of appropriate propulsion from their passive prostheses, amputees rely on different gait strategies than non amputees (7); suggesting that amputees can benefit from powered prostheses capable of providing power in both DP and IE with impedance modulation similar to the human ankle.

While physical systems interact with each other, they are behaving either as an impedance (e.g. Accepts external motion inputs and generates force outputs) or an admittance (e.g. Accepts external force inputs and generates motion outputs) (102). The coupled mechanical systems must physically complement each other, meaning that in any degree of freedom, if one system is an impedance, the other system has to be an admittance (102). During gait, at the moment the heel interacts with the ground (heel-strike) the ankle is being manipulated by the environment since the ankle accepts the external force and generates the appropriate motion, so it may be considered as a system in admittance. At push off, the ankle manipulates the 
environment, generating the necessary torques to produce the required motion, and so it may be considered as impedance. This suggests that ankle-foot prostheses should use an admittance controller at heel -strike and an impedance controller at push off. In general, an impedance controller uses position encoders mounted in the actuators to determine the position of the robot end effector. The controller uses the desired and feedback positions to generate the actuators' desired torques. Torque sensors are used to provide the means for estimation of the external torque feedback that, along with the desired torque, are used to define the appropriated input to the motors (103). An admittance controller or position based impedance controller uses the environment torque feedback to estimate the appropriate actuator position. The desired actuators' position and position feedback are used to estimate the appropriate actuators inputs (103). In other words, the impedance controller accepts external motion inputs and generates output torques, while the admittance controller accepts external torque inputs and generates output motions.

The mechanical impedance of the human ankle has been studied by many researchers $(48-51,53)$. Quasi-static stiffness of the ankle in the sagittal plane has been used in the design of ankle-foot prostheses capable of producing a positive work during the gait. Sup et al. developed a knee and ankle prosthesis capable of controlling the impedance of both the knee and ankle joints in the sagittal plane by controlling the neutral position of the foot during the gait (25-28). BiOM ${ }^{\circledR}$ is a commercially available ankle prosthesis that is capable of providing the necessary energy during push-off (plantarflexion); therefore, actively contributes in gait and lowers the gait metabolic cost by $8.9 \%$ to $12.1 \%$ at different gait speeds compared to a passive prosthesis (34). The controller in both of the aforementioned prostheses use a finite state machine to identify the gait phase and estimate the appropriate ankle torques. This allows the prostheses to adapt to different gaits scenarios such as different cadence or walking uphill and downhill (8). Although the aforementioned prostheses improve the gait of amputees, they are designed to modulate the ankle torques in the sagittal plane only. 
In this paper the concept and prototype of a multi-axis powered ankle-foot prosthesis capable of controlling two degrees of freedom is presented. Additionally, the paper describes the methods to measure torque feedback in both DP and IE using strain gauges; the preliminary steps toward development of impedance and admittance controllers based on the torques and positions feedback; the development of a finite state machine to identify the state of the gait and switch between admittance and impedance controllers, and preliminary tests to evaluate the performance of the prosthesis while walking on a circular treadmill as an evaluation platform.

\subsection{CABLE-DRIVEN POWERED ANKLE-FOOT PROSTHESIS WITH TWO CONTROLLABLE DEGREES OF FREEDOM}

A multi-axis ankle prosthesis may enhance gait efficiency by extending the control of IE during walking in a straight line and turning. This novel design is aimed to enable the device to adapt to uneven and inclined ground surfaces and allow the amputees to benefit more from their prostheses rather than using their hip joint as a compensatory gait mechanism; enabling a more agile and natural gait with less stress on other joints.

A prototype design of a cable-driven ankle-foot prosthesis controllable in both DP and IE was designed and fabricated in an effort to study the feasibility of the steering and maneuverability with a 2 degrees of freedom (DOF) ankle joint (Fig. 8.1). The design allowed for the range of motion (ROM) and angular velocity similar to the human ankle during straight walking and turning while producing enough torque for propulsion. 


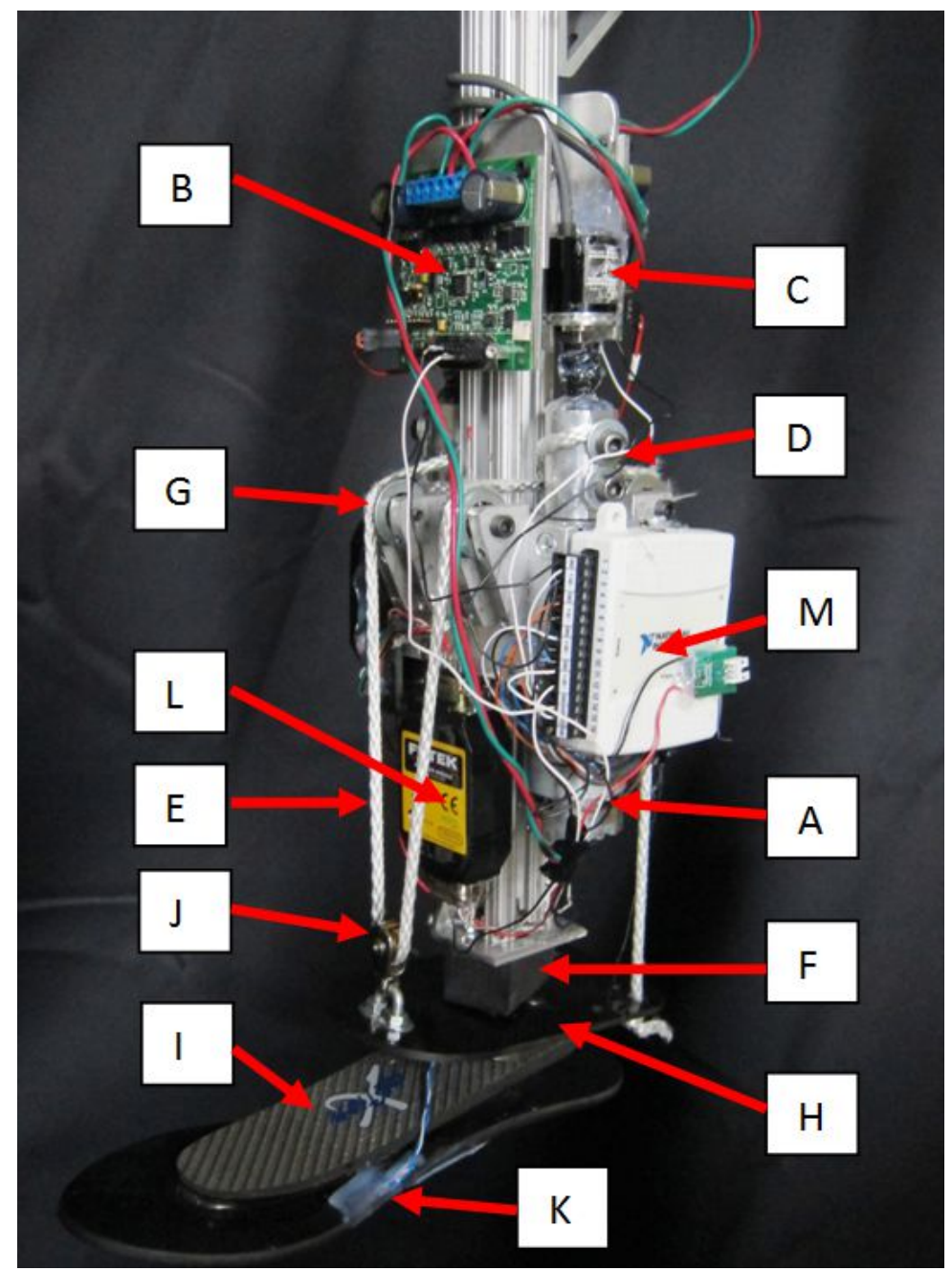

Figure 8.1. Two-DOF ankle-foot prosthesis prototype.

The device consists of two DC motors and planetary gear heads (A) powered by two motor controllers (B) connected to two quadrature encoders (C). Two cable drums (D) transfer the required torque to the ankle through the shock-absorbing nylon rope (E). A universal joint (F) connects the pylon to the foot and is surrounded by an elastomer to provide passive stiffness and damping to the ankle. Both actuators apply the torque to the foot using a cable-driven mechanism with pulleys $(\mathrm{G})$. The cable is attached to a carbon fiber plate $(\mathrm{H})$, which is connected to a commercially available prosthetic foot (Otto Bock Axtion $\left.{ }^{\circledR}\right)$ (I). In the rear side of the carbon fiber plate, the cable is mounted to both sides of the longitudinal axis of 
the foot. At the front side of the carbon fiber plate, the cable passes through a pulley (J). Torque feedback is provided by six strain gauges in the foot ( $\mathrm{K}$ and Fig. 8.2) using two strain gauge amplifiers (L). An analog to digital converter (M) is connected to a remote computer and is used to acquire the sensors data and provide the motor controllers' inputs. The mechanism is capable of moving in DP when the motors rotate in opposite directions and in IE when the motors rotate in the same direction. Also, any combination of DP and IE can be obtained by combining different amounts of rotations in each motor.

\subsection{ANKLE TORQUE AND ANGLE FEEDBACK}

To develop impedance and admittance controllers, force and position feedbacks are required. The angular displacement of the ankle in DP and IE $\left(\theta_{D P}\right.$ and $\theta_{I E}$ respectively) can be calculated from the left and right quadrature encoders' feedback ( $\theta_{\text {Left }}$ and $\theta_{\text {Right }}$ respectively), where $K_{D P}$ and $K_{I E}$ are constant gains to define the ankle rotations as a function of the quadrature encoders' outputs, and are based on the prosthesis' geometry.

$$
\begin{aligned}
& \theta_{D P}=K_{d p}\left(\frac{\theta_{\text {Left }}+\theta_{\text {Right }}}{2}\right) \\
& \theta_{I E}=K_{\text {ie }}\left(\theta_{\text {Right }}-\theta_{\text {Left }}\right)
\end{aligned}
$$

The torque feedback was estimated using strain gauges, as they are commonly used on load cells to measure the strains of structures due to external forces. The strain gauges change the resistance as they stretch or contract and their change in resistance can be correlated to the strain of the object they are attached to and consequentially the force or torque applied to the object. Strain gauges are typically wired in a Wheatstone Bridge configuration using four strain gauges (some of the strain gauges can be replaced by resistors). The increase or decrease in resistance of two of the strain gauges (placed in opposite sides of the bridge) causes the output of the bridge to increase or decrease, respectively. The opposite is also true for the 
other two strain gauges where the increase or decrease in resistance decreases or increases the output voltage of the bridge, respectively.

For estimating the torque in DP, four strain gauges were attached to the sole of the foot (Fig. 8.2-A). The strain gauges $S_{d p} 2$ were located behind the center of rotation of the ankle in DP and were wired into opposite sides of the Wheatstone Bridge (Fig. 8.3-A). Any ground reaction force at the hill caused a decrease in voltage of the Wheatstone Bridge, which could be correlated to the torque in DP of the foot when the heel was interacting with the ground (e.g. Heel-strike). The strain gauges $S_{d p} 1$ were located in front of the center of rotation of the ankle in DP and wired in opposite sides of the Wheatstone Bridge (Fig. 8.3-A). Any ground reaction force from the ground at the front of the foot caused an increase in the output voltage of the Wheatstone Bridge, which could be correlated to the torque in DP of the foot when it was contacting the ground (e.g. Push off). Note that when the foot was flat on the ground, the output from the strain gauges $S_{d p} 1$ cancel the output from the strain gauges $S_{d p} 2$; therefore, the resultant voltage could always be correlated to the net DP torque in the ankle.

For estimating the torque in IE, two strain gauges were attached to the top of the foot as seen in Fig. 8.2-B. The other two strain gauges were attached to an inert piece of carbon fiber, but could also be replaced by two resistors with resistance identical to the strain gauges. The strain gauges were placed on the outside edges of the foot and were on the same side of the Wheatstone Bridge (Fig. 8.3); hence, the difference in strains caused an increase or decrease in voltage at the bridge. The voltage can be correlated to the IE torque in the foot when the front of the foot is contacting the ground (e.g. Push off). This configuration, made the Bridge insensitive to the torque in DP, since the strain gauges were in the opposite ends of the bridge. Therefore, if they both contract or stretch by the same amount as it would happen in the presence of a DP torque, the output would not be affected. This 
feature is important since it is necessary to have the IE torque estimation to be decoupled from the DP torque.

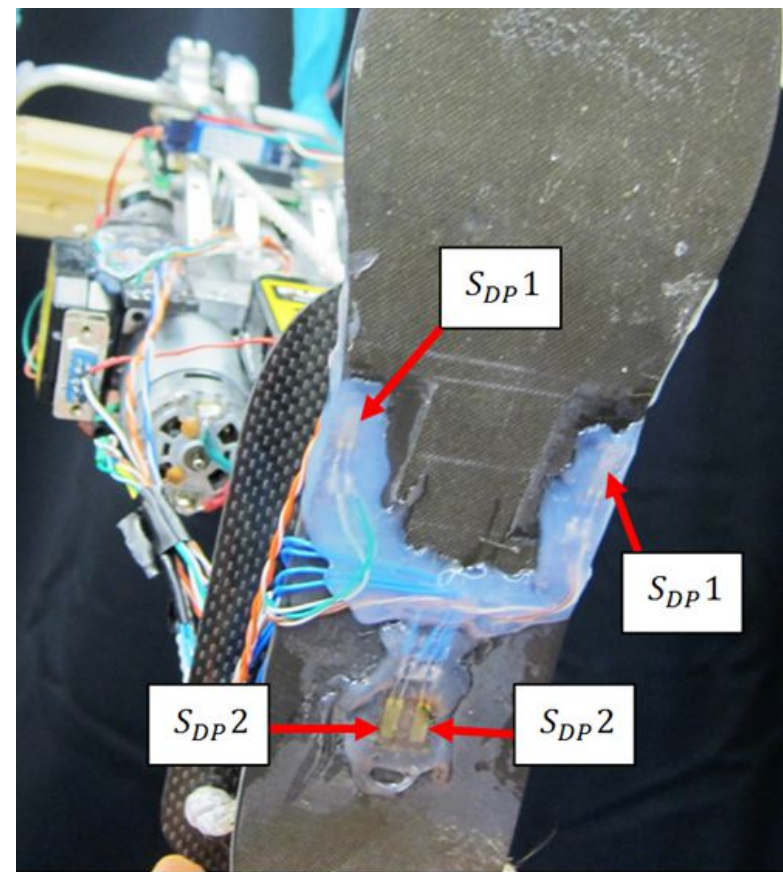

(A)

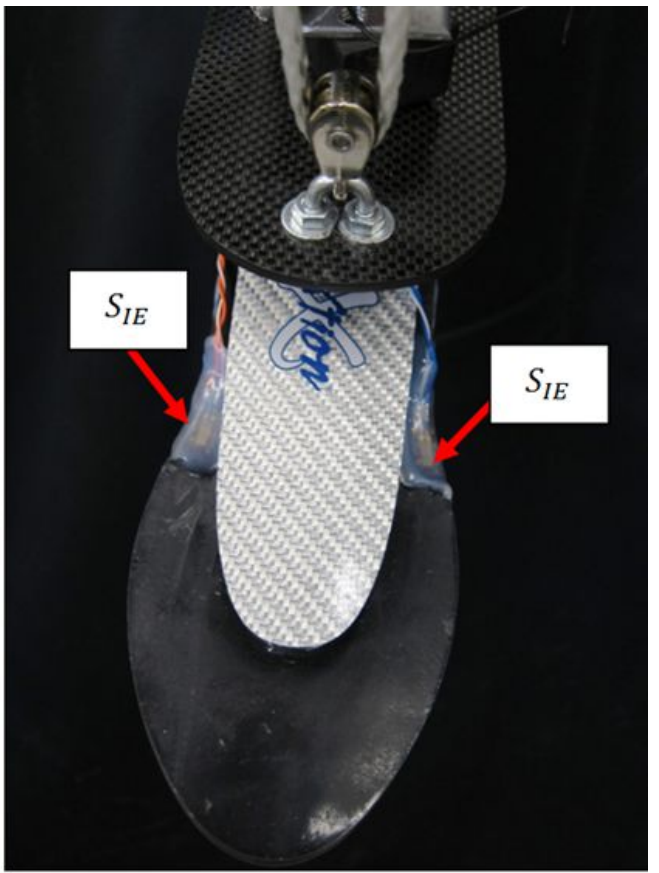

(B)

Figure 8.2. Strain gauge placement in the prosthesis. A: For DP torque two strain gauges are used for push-off torque estimation $\left(\mathrm{S}_{\mathrm{DP}} 1\right)$ and two strain gauges are used for heel-strike torque estimation $\left(\mathrm{S}_{\mathrm{DP}} 2\right)$. B: For IE torque two strain gauges are used for push-off torque estimation $\left(\mathrm{S}_{\mathrm{IE}} 1\right)$.

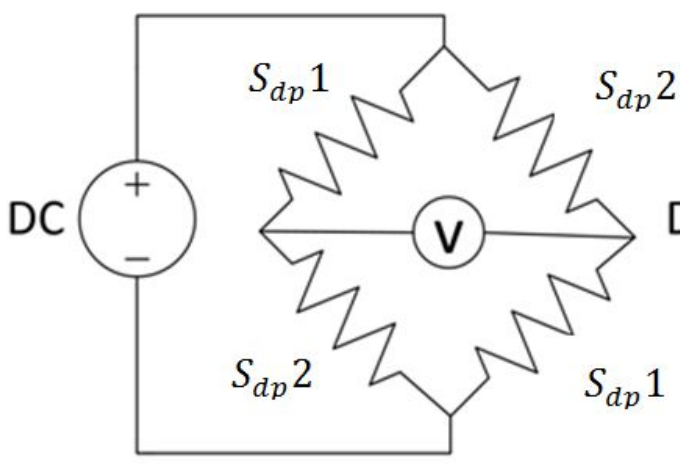

(A)

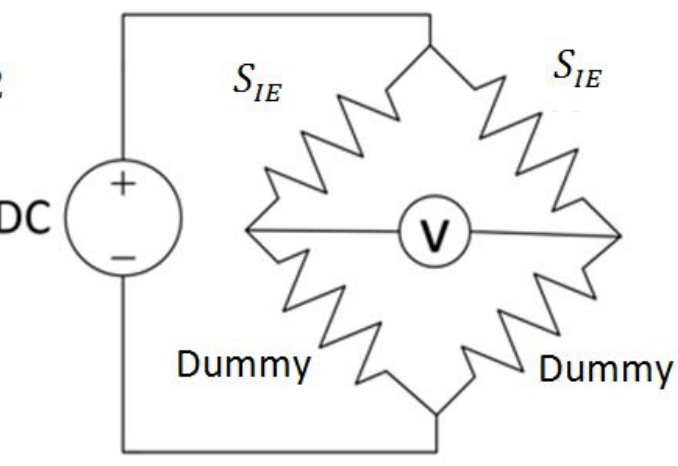

(B)

Figure 8.3: A: Strain gauge placement in the Wheatstone Bridge for DP torque estimation. Two strain gauges are used for push-off DP torque estimation $\left(S_{D P} 1\right)$ and two strain gauges are used for DP heel-strike torque estimation $\left(S_{D P} 2\right)$. B: Strain gauge placement in the Wheatstone Bridge for IE torque estimation. Two strain gauges are used for push-off IE torque estimation $\left(S_{I E}\right)$. 
To correlate the strain gauge readings to the actual disturbance torques, a Kistler ${ }^{\circledR}$ Type 5233A force plate was used to measure the external force applied during static loading tests. The tests consisted of loading the foot in different configurations and recording the applied force and the corresponding strain measurement. The tests were: plantarflexion by applying a load when the heel was in contact with the ground, dorsiflexion by applying a load when the forefoot was in contact with the ground, eversion by applying a load when the right edge of the forefoot was in contact with the ground, and inversion by applying a load when the left edge of the forefoot was in contact with the ground. From the external forces, the geometry of the foot, and the strain measurements, the applied torques were calculated. It is important to note that for DP, the proportional factor between the external force and the strains measured at heel loading and forefoot loading were not the same, since the strain gauges were attached to two different areas of the prosthetic foot. The proportional factors for the strain gauges at heel loading and forefoot loading were estimated as $1.41 \mathrm{Nm} /$ volt and $19.52 \mathrm{Nm} /$ volt, respectively. As a result, different proportional factors needed to be used depending if the strain measurement was positive or negative. For IE, the proportional factors for inversion and eversion torques were closer (4.43 Nm/volt and $3.55 \mathrm{Nm} /$ volt, respectively), which was expected since the foot is near symmetrical about its sagittal plane.

\subsection{CONTROLLER}

\subsubsection{Finite State MaCHINE}

A finite-state machine was designed to switch between impedance and admittance controller (Fig. 8.4). The finite-state machine uses pre-recorded timehistory of the ankle angles in DP and IE during normal walk and real time torque feedback from the strain gauges to switch between the states. The recorded ankle angles of an unimpaired human subject were measured using a motion capture camera system. It is aimed to use the trajectories of the ankle of unimpaired human beings as a start point for a powered prosthesis, which can be tuned to the specific 


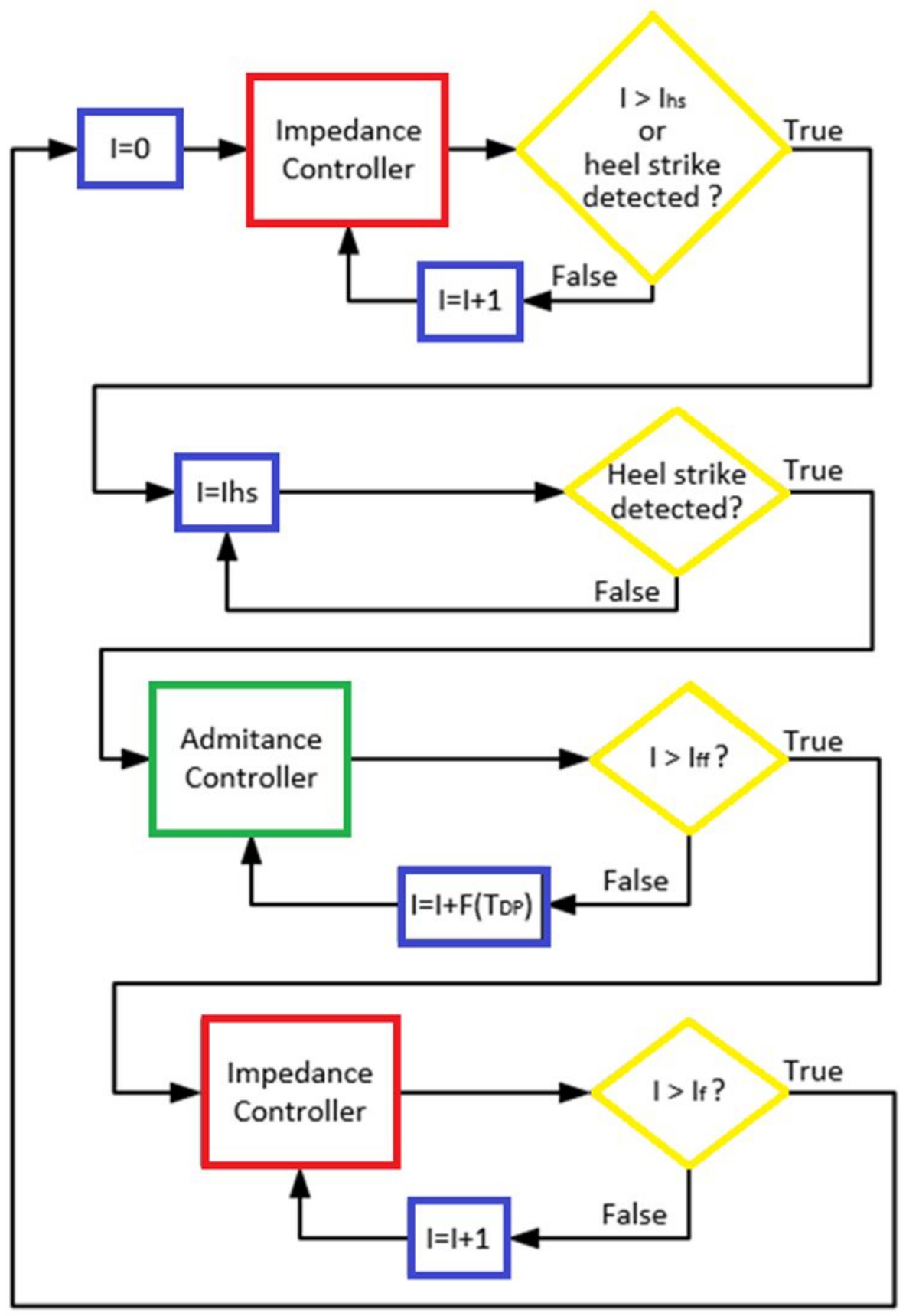

Figure 8.4. Finite-state machine to switch between impedance and admittance controllers. In the admittance controller the increment of the index (I) is a function of the external torque disturbance $T_{d p}$. Once the foot reaches flat foot, the control switches back to an impedance controller until the foot reaches the middle of the swing phase where the index I is reset to zero and the cycle starts again. Note that in admittance control the increment of the index (I) is a function of the external torque disturbance $T_{d p}$, which will be explored later. 
needs of amputees. The ankle angles were accessible as look-up data table to the estate machine and controllers. The vectors with the ankle data started and finished in the middle of the swing phase (vector indices $I_{0}$ and $I_{f}$, respectively). Also the index for the data at the beginning of the flat foot (index $I_{f f}$ ), and expected heelstrike (index $I_{h s}$ ) were known. These points were important as the finite-state machine needed to switch from impedance controller to admittance controller at heel-strike and from admittance controller to impedance controller at the initiation of the flat foot phase.

From Fig. 8.4 it can be seen that the foot starts at the middle of the swing phase and moves with the active impedance controller to the expected heel-strike. If heelstrike is detected before it is expected (e.g. The user started to walk faster) the finite state machine skips the rest of the swing phase and starts the heel-strike phase immediately with the admittance control. If it does not detect a heel-strike (e.g. The user started to walk slower or has stopped), the prosthesis will advance to the angle at the beginning of the heel-strike phase and will hold that position until heel strike is detected. This is important as the robot can adjust to small variations in walking speed, and will start and stop automatically when the gait starts or stops.

\subsubsection{IMPEDANCE CONTROLLER}

The robot has two DC motors working together to produce torques that move the foot with respect to the pylon. When the motors rotate in opposite directions DP motion is produced. When they move in the same direction IE motion is produced. This implies that two controllers are needed, one for each motor.

The impedance controller (Fig. 8.5) for both the left and right motors uses position encoders mounted in their respective gear boxes to determine the position of the foot. The controller uses the desired and feedback positions to derive the actuators desired torques using a PD controller. The torque feedback from the strain gauges are used to estimate the ground reaction torques to be used as the feedback. The torque feedback gain $\mathrm{K}$ adjusts the quasi-static stiffness of the ankle, which will 
be explored later. The desired torque and torque feedback are then used to derive the appropriate control input to the motors using a PD controller. Note that the reference angle for the left motor controller (looking at the prosthesis from the front) is the sum of the DP and IE angles, while the reference angle for the right motor controller is the difference between DP and IE angles. Similarly, the torque feedback for the left motor controller is the sum of the DP and IE ground reaction torques, while the feedback torque for the right motor controller is the difference between DP and IE environment torques. This is necessary since the outputs (both angle in torque) of the prosthesis in DP are proportional to the output of both motors, and the outputs in IE are the difference between the output of the motors.

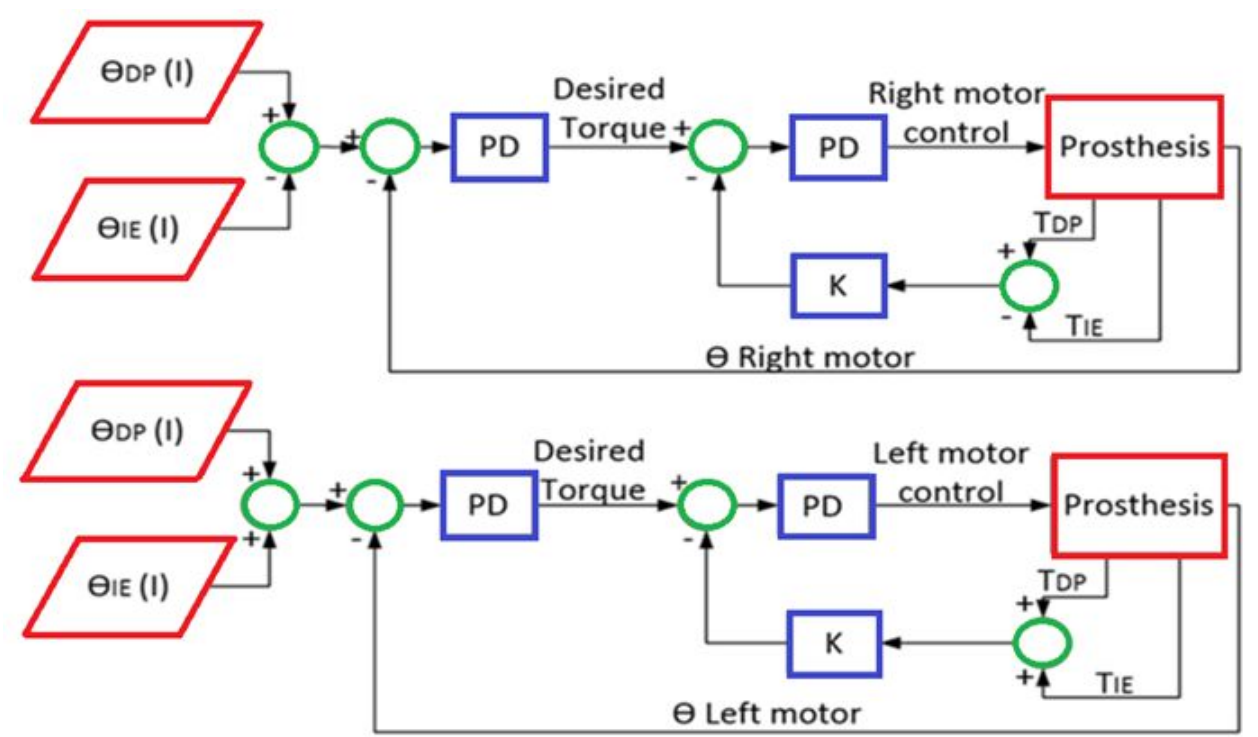

Figure 8.5. Impedance controllers for the left and right motors. The reference angle for the left motor controller is the sum of the DP and IE angles, while for the right motor controller is the difference between DP and IE angles. The torque feedback in the left motor controller is the sum of the DP and IE torques, while for the right motor controller is the difference between DP and IE torques.

\subsubsection{Admittance Controller}

An admittance controller requires torque feedback to update an inner position controller. The proposed admittance controller was designed to use a look-up data table for updating the inner position control (Fig. 8.6). The control integrates the 
ground reaction torque feedback (in DP) to increase the index of the look-up table of the ankle angles proportionally to the external torque. This way, an external torque input will make the prosthesis able to advance through the data vector, while the absence of an external torque will keep the foot stationary. This allows the foot to follow the prerecorded angular trajectories, while admitting external torque to produce motion. Also, at heel-strike the foot will not move unless it contacts the ground; therefore, the foot will start and stop moving automatically based on the external torque feedback. It is important to note that this controller will only engage when the motion of the device is known, and the external torque is only used to control how fast the robot will follow the predetermined trajectory. At this point the prosthesis does not have IE torque feedback at the heel, so only the DP torque was used to update the controller at heel strike.

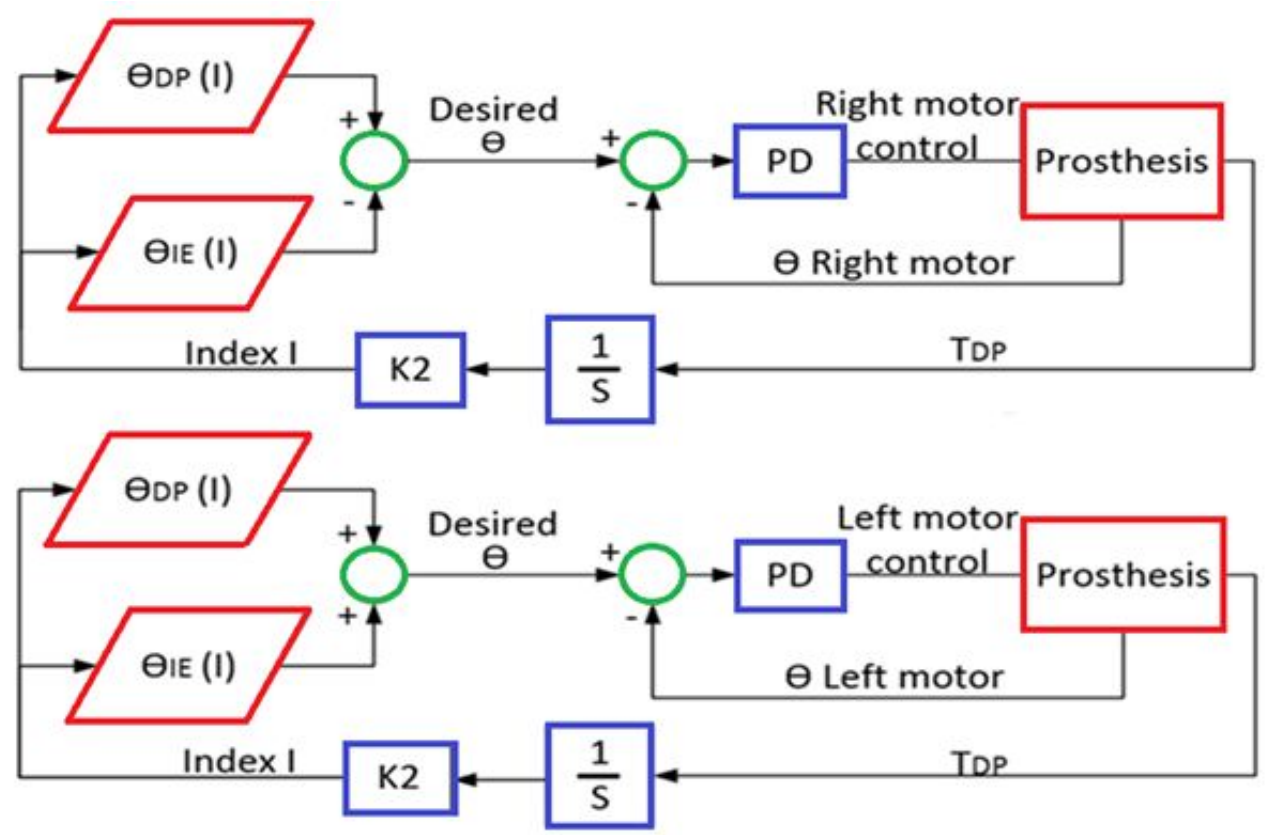

Figure 8.6. Admittance controllers for the left and right motors. The reference angle for the left motor controller is the sum of the DP and IE angles, while for the right motor controller is the difference between DP and IE angles. The admittance comptroller uses the torque feedback in DP to update the lookup table index I. 


\subsection{PRELIMINARY EVALUATION EXPERIMENTS}

\subsubsection{IMPEDANCE CONTROLlER IN QUASI-STATIC CONDITION}

To evaluate the impedance controller and its ability to change the quasi-static stiffness of the ankle, an experiment was designed to record the quasi-static torqueangle relationship of the prosthesis. The prosthesis was attached to an Anklebot, a lower extremity therapeutic robot (Interactive Motion Technologies, inv.) As seen in Fig. 8.7. The Anklebot is capable of applying torques and record angular motion of the ankle in both DP and IE, this makes it suitable for the experiment. To test the DP stiffness, the prosthesis impedance controller was set at a reference angle of zero degrees and a constant torque feedback gain $\mathrm{K}$ for each test. Six tests were performed setting the gain $\mathrm{K}$ at different values ranging from -0.5 to 1.5 . In each test the Anklebot moved the foot from the equilibrium point to $6^{\circ}$ dorsiflexion and followed by moving the foot to $6^{\circ}$ plantarflexion. The movement speed was set to $5 \%$ second and the data at the encoders were recorded at a sampling rate of 200 samples per second. The results were filtered with $0.5 \mathrm{~Hz}$ cutoff frequency to remove the sensor noise.

The results of the tests with different gains are shown in Fig. 8.8, depicting the unloading, transition, and loading phases of the ankle. It can be seen that the change in the feedback gain effectively changed the stiffness of the ankle in DP (or the slope of torque-angle curve in Fig. 8.8). Zero gain caused the prosthesis to behave as a passive prosthesis, since it is not a backdrivable mechanism. Negative gains caused the prosthesis stiffness to increase compared to the zero gain test. Positive gains resulted in a decrease in the prosthesis stiffness compared to the zero gain case. All the gains produced near linear changes in DP torque with respect to the change in angle, with some deviation near the origin caused by the transition in the ankle from loading to unloading and its effects on the bending of the composite plate. Best fit lines were fit to each of the tests in Fig 8.8, and the slopes (stiffness of the ankle) of these lines were plotted against their respective gains in Fig. 8.9. It can 
be seen that there is a near linear relation between the change in torque feedback gain and the quasi-static stiffness of the prosthesis with positive gains. The stiffness of the prosthesis in DP was found to be $2.09 \mathrm{Nm} /$ degree with a -0.5 gain that decreased to $0.92 \mathrm{Nm} /$ degree at gain 1.5.

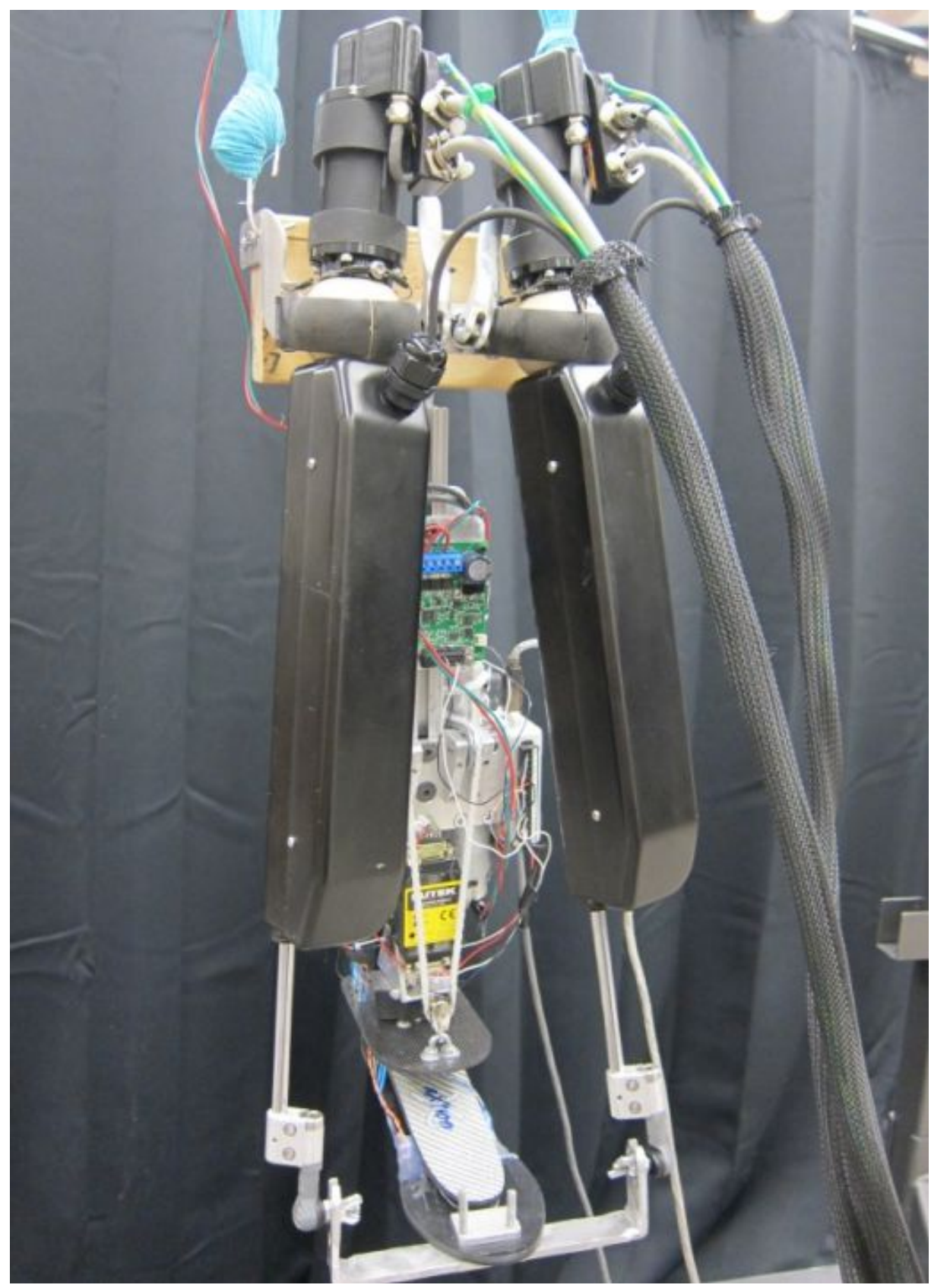

Figure 8.7. Ankle-foot prosthesis attached to the Anklebot. 


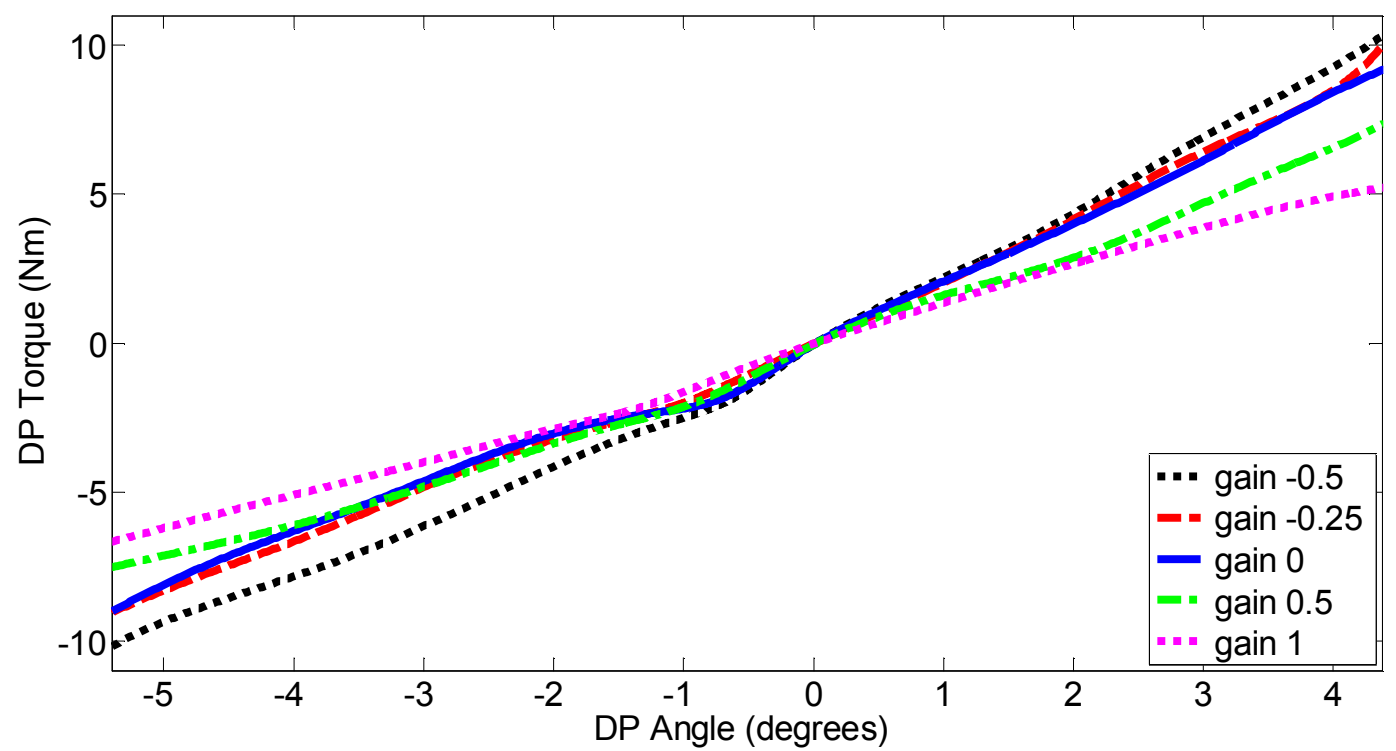

Figure 8.8. DP torque-angle relationship in the prosthesis with impedance control and different torque feedback gains. Negative angles are plantarflexion, positive angles are dorsiflexion.

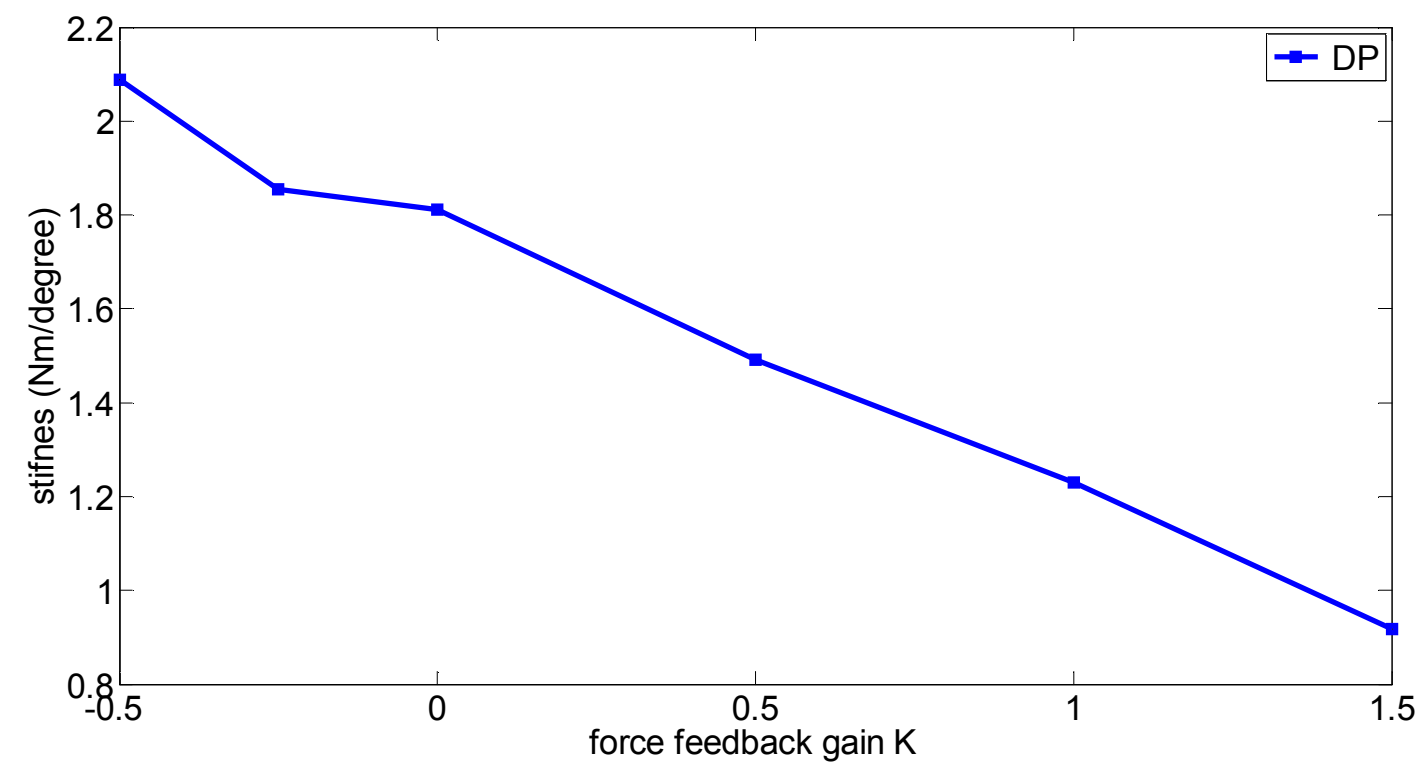

Figure 8.9. Ankle stiffness in DP at different DP torque feedback gains.

To test the IE stiffness a similar experiment was conducted. Six tests were performed with the same torque feedback gains as the DP test. In each test the Anklebot moved the foot to $12^{\circ}$ eversion from the equilibrium point and in a 
continuous motion returned the foot to $12^{\circ}$ inversion. Large angular displacements were needed in the IE test since, by design, the ankle-foot prosthesis shows a smaller passive stiffness in IE than in DP. It can be seen in Fig. 8.10 that the change in the feedback gain effectively changed the stiffness of the ankle in IE. Similar to the DP test, negative gains caused the prosthesis stiffness to increase compared to the zero gain test. With positive gains, the prosthesis stiffness decreased compared to the zero gain tests. All the gains produced near linear changes in IE torque with respect to the change in angle, with some deviation near the origin caused by the transition in the ankle from loading to unloading. Similar to DP tests, the quasistatic stiffness of the ankle were plotted against their respective gains, as shown in Fig. 8.11, indicating a near linear relation between the change in torque feedback gain and the quasi-static stiffness of the prosthesis for the positive gain. The prosthesis stiffness in IE was found to be $0.53 \mathrm{Nm} /$ degree at a -0.5 gain that decreased to $0.17 \mathrm{Nm} /$ degree at gain 1.5.

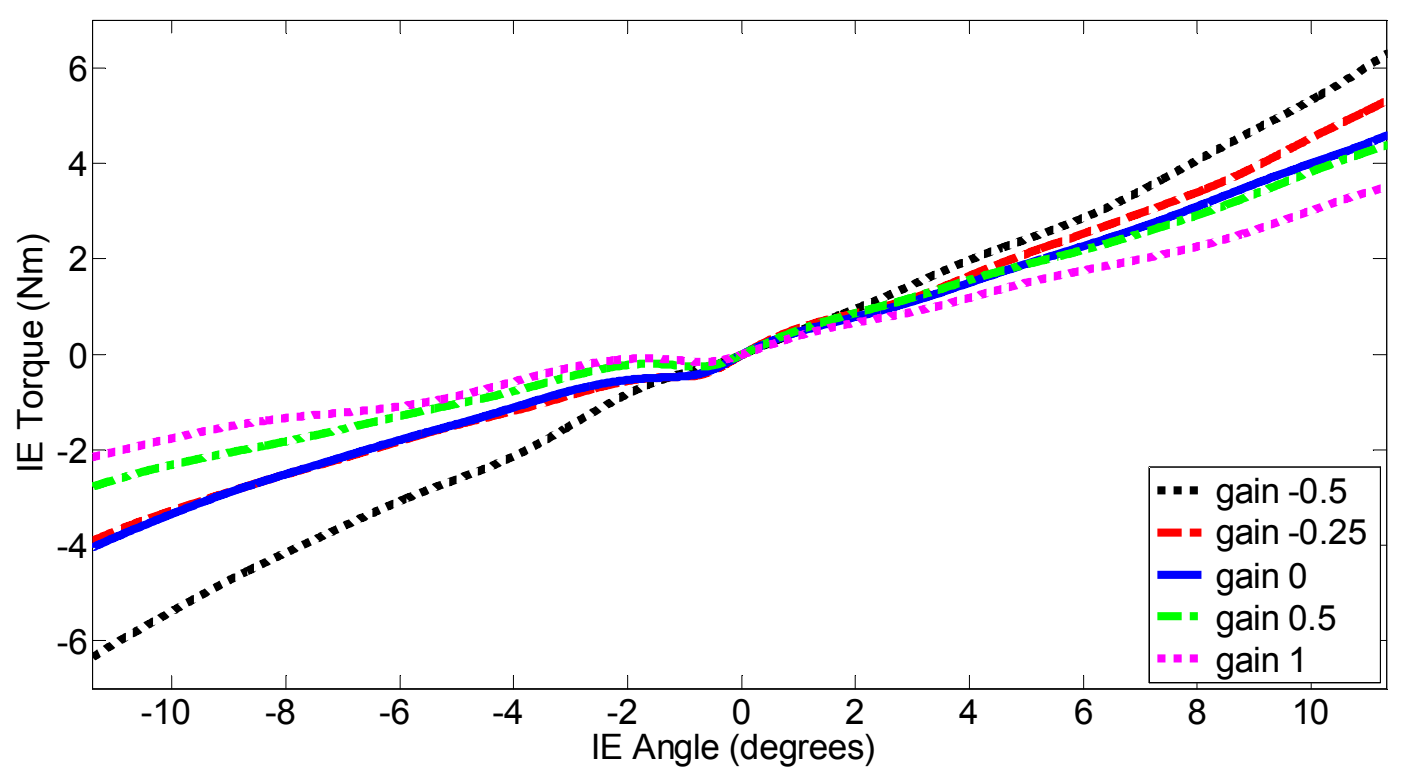

Figure 8.10. IE torque-angle relationship in the prosthesis with impedance control and different torque feedback gains. Negative angles are plantarflexion, positive angles are dorsiflexion. 


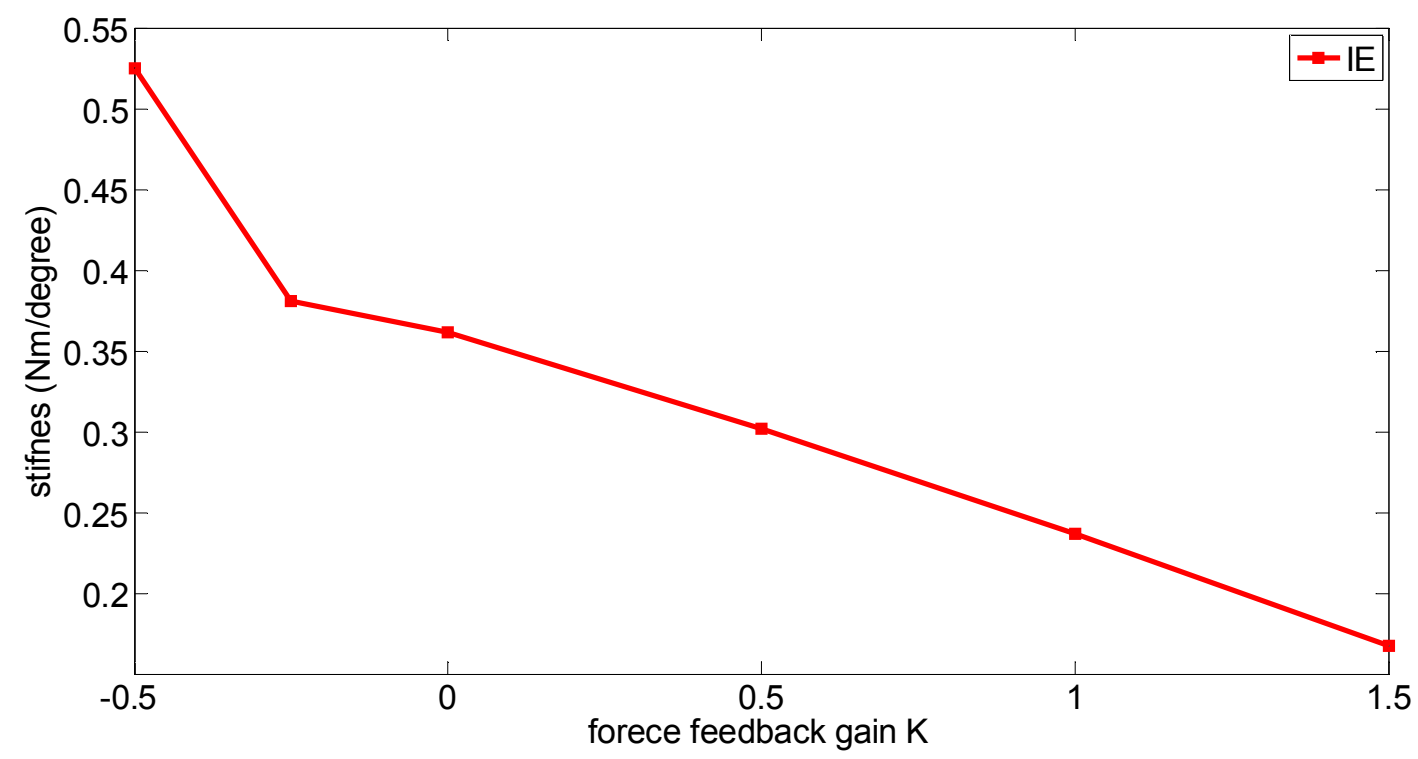

Figure 8.11. Ankle stiffness in DP at different DP torque feedback gains.

\subsubsection{Prosthesis Test ON The Circular Treadmill}

The circular treadmill was used to test the prosthesis performance with the impedance/admittance control and compare the results with the performance of the device using a position control and no control (passive prosthesis). The impedance/admittance controller was set with a torque feedback gain 0.5 for both DP and IE. The load on the foot was equivalent to $22.8 \mathrm{Kgf}$. The radius of turn during the walk was set to $0.85 \mathrm{~m}$. The position controller used a PD controller to follow the trajectory of the previously recorded data of a human subject ankle in both DP and IE. With all the controls off, the device behaved as a passive prosthesis with the stiffness equivalent to gain zero as shown in Figs. 8.8 and 8.10. During the tests the ground reaction forces were obtained from the strain gauges readings and used to estimate the resultant torque applied to the ankle (Figs. 8.12 and 8.13). It was seen that during the swing phase there were zero torque feedbacks since the foot was not contacting the ground. When contact happened, the passive prosthesis showed the largest reaction torques which saturated the data acquisition system at $15 \mathrm{Nm}$ torque. The position controller decreased the DP torque at heel-strike, but 


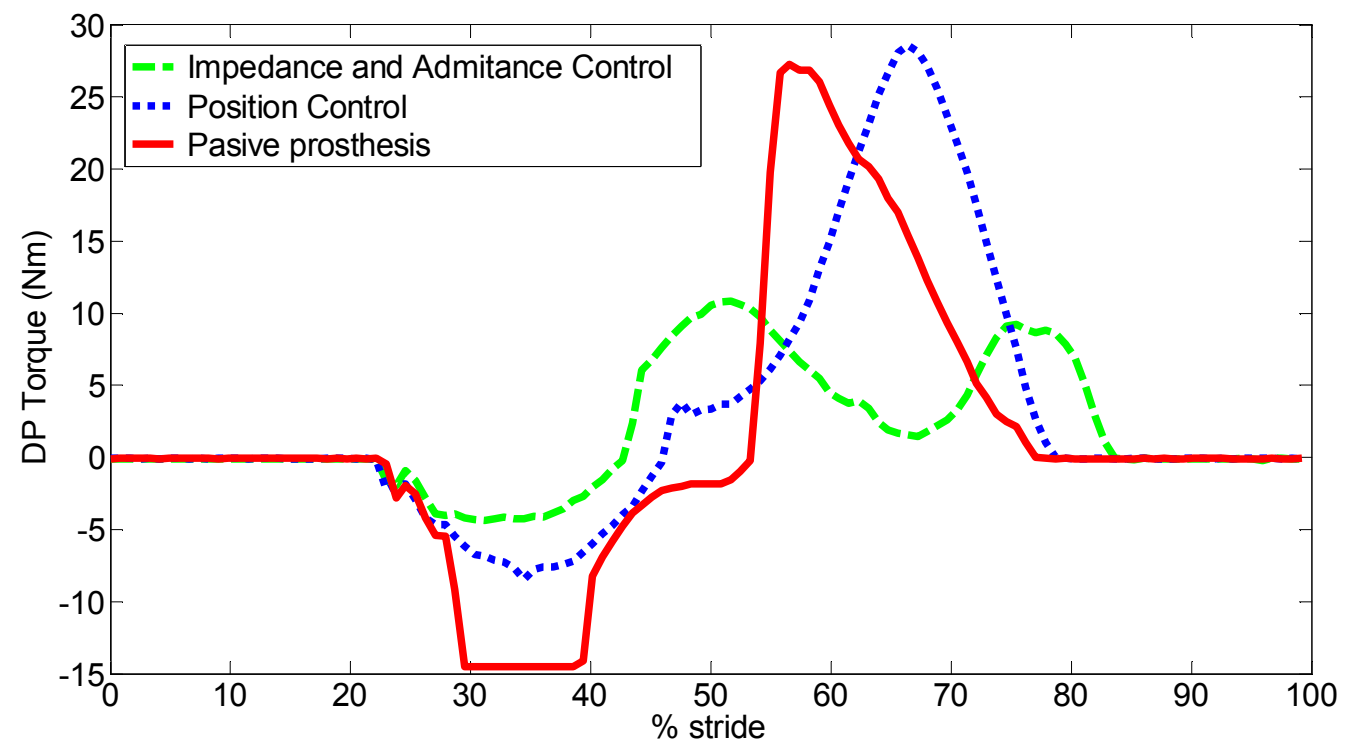

Figure 8.12. Ankle external torque in DP during a representative gait cycle with different control strategies. Negative torques induces plantarflexion (heel-strike) and positive torques induces dorsiflexion (push off). With the passive prosthesis, the data acquisition system saturates during heel-strike at $-15 \mathrm{Nm}$ torque.

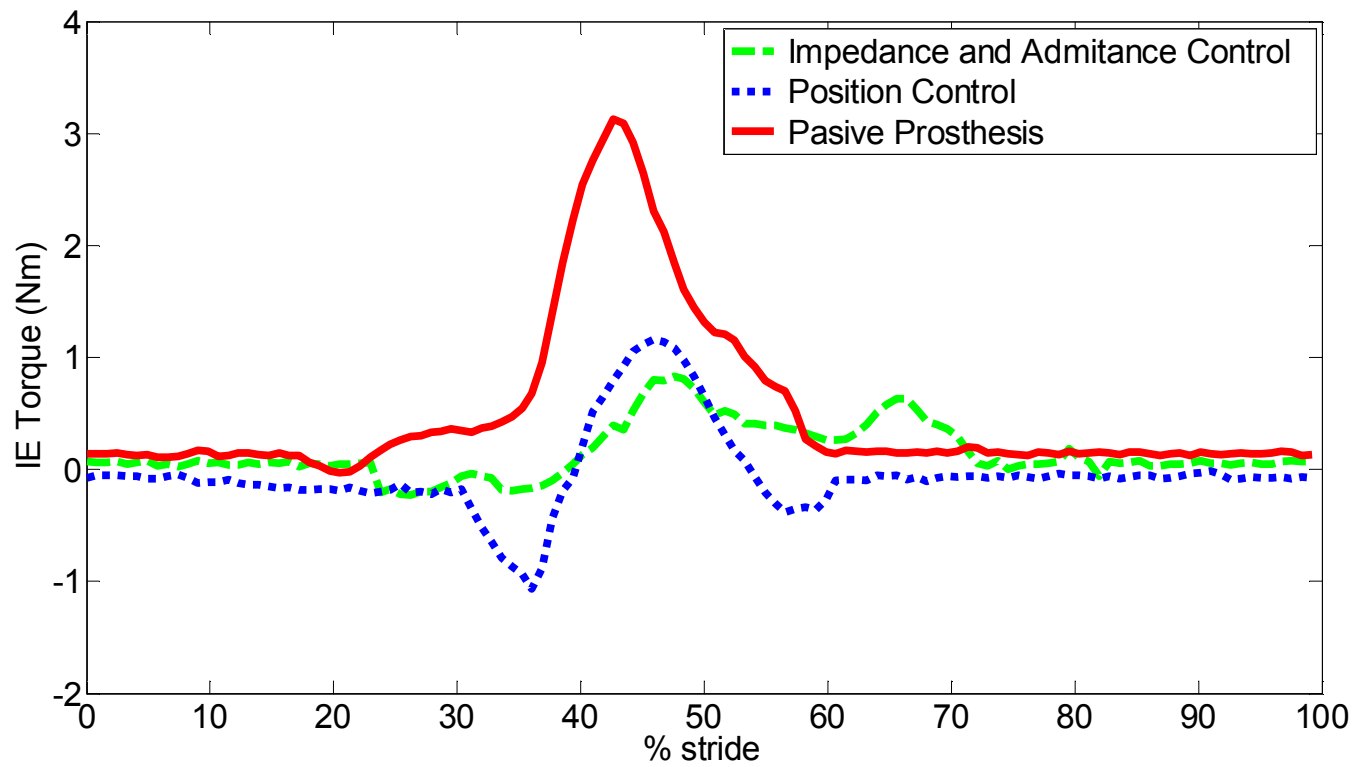

Figure 8.13. Ankle external torque in IE during a representative gait cycle with different control strategies. Negative torques induces eversion and positive torques induces inversion. 
showed similar torque at push-off when compared to the passive prosthesis. The impedance/admittance control showed the least amount of DP torques both at heelstrike and push off. IE torques were the largest in the passive prosthesis and the impedance/admittance controller showed the least amount of torque. Inversion torques were larger than eversion torques for all experiments, which is expected since the foot is turning left as it walks on the treadmill, putting pressure in the inside edge of the foot.

It is seen in Figs. 8.12 and 8.13 that the impedance/admittance controller was capable of reducing the amount of external torque in the foot in both DP and IE; however, it increased the amount of time the foot was in contact with the ground. This is expected since the impedance controller is effectively changing the stiffness of the ankle by applying a torque in the same direction as the disturbance torque. This causes the foot to be at a larger dorsiflexion angle compared to the reference input, resulting in an extended time for push-off.

Smaller reaction forces at heel-strike are desirable as these forces are directly transferred to the user. At push-off, smaller forces may reduce the energy consumption of the prosthesis. However, the prosthesis needs to have enough stiffness at heel strike to control the impact and generate enough torque for forward propulsion during push-off. The impedance/admittance control was capable of reducing the external reaction torques, but this is only desirable if the torques are large enough to follow the desired trajectory. The input and output trajectories of the foot in both DP and IE during the tests can be seen in Figs. 8.14 and 8.15, respectively. The input data is the time history of rotations of a human ankle during gait, and the output is the trajectory of the ankle obtained from the quadrature encoders in the prosthesis. For ease of comparison, the output plots have a time shift to remove the 75 milliseconds delay of the output. From Fig. 8.14, it can be seen that the impedance/admittance controller input held the ankle constant for near $40 \%$ to $65 \%$ of the stride, due to the state machine reaching the index of the expected heel-strike, but heel-strike has not happened yet. The impedance/admittance 
controller was capable of more closely tracking the reference trajectory compared to the position control, since it accounts for the external torques in the control. In IE (Fig. 8.15), the tracking performance of both controllers decayed compared to the performance in DP. Due to the physical characteristics of the prosthesis, small angular differences between the left and right motors caused larger changes in the foot rotations, making the system more sensitive to disturbances and noise compared to DP.

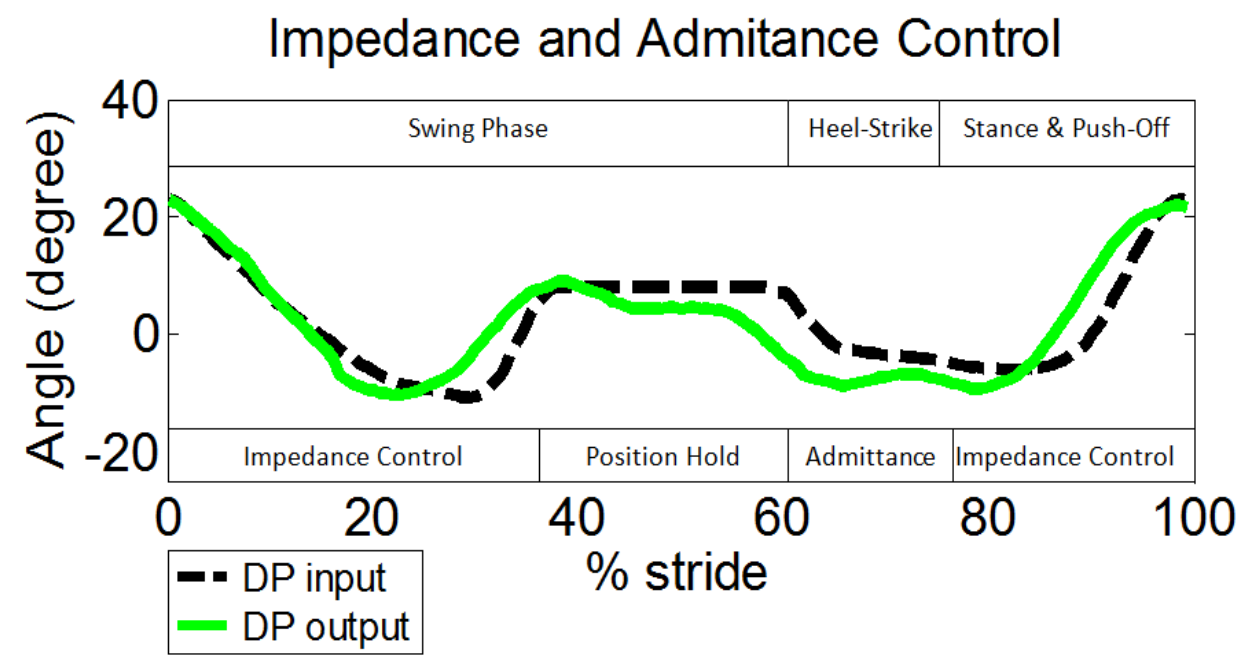

\section{Position Control}

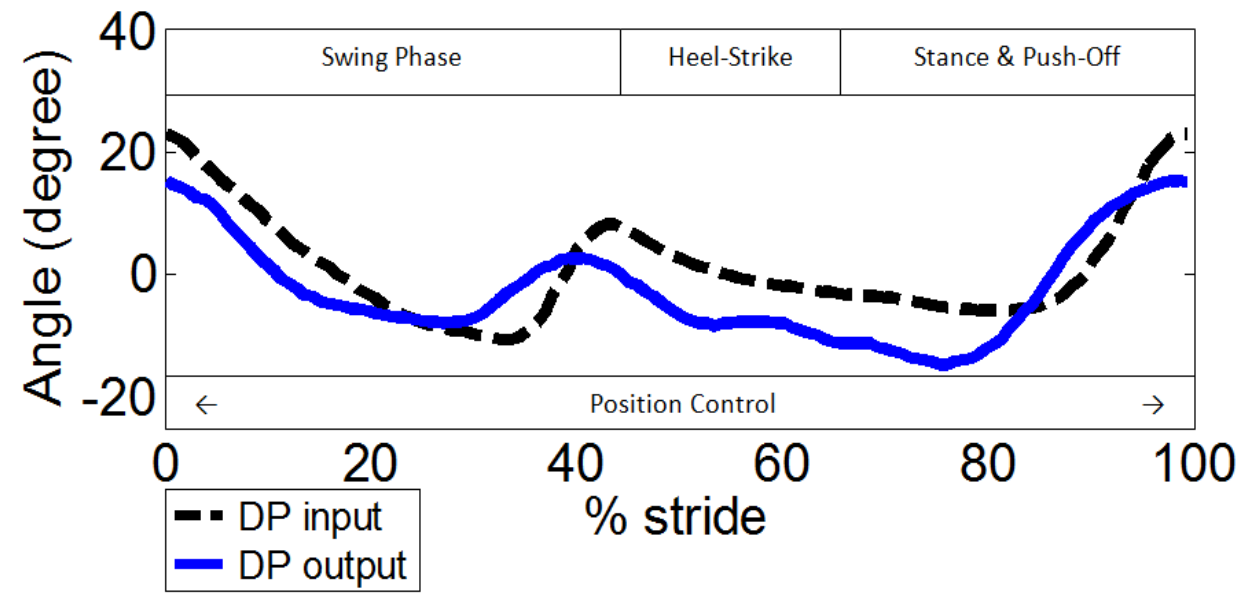

Figure 8.14. Input and output (compensated for 75 milliseconds time delay) of the ankle trajectory in DP during a representative gait cycle with admittance and impedance control (top) and position control (bottom). 
Impedance and Admitance Control

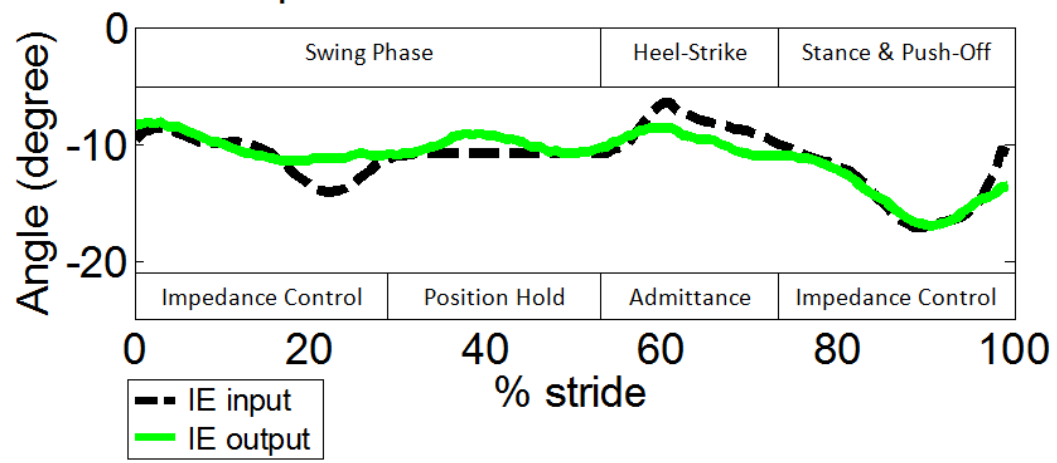

Position Control

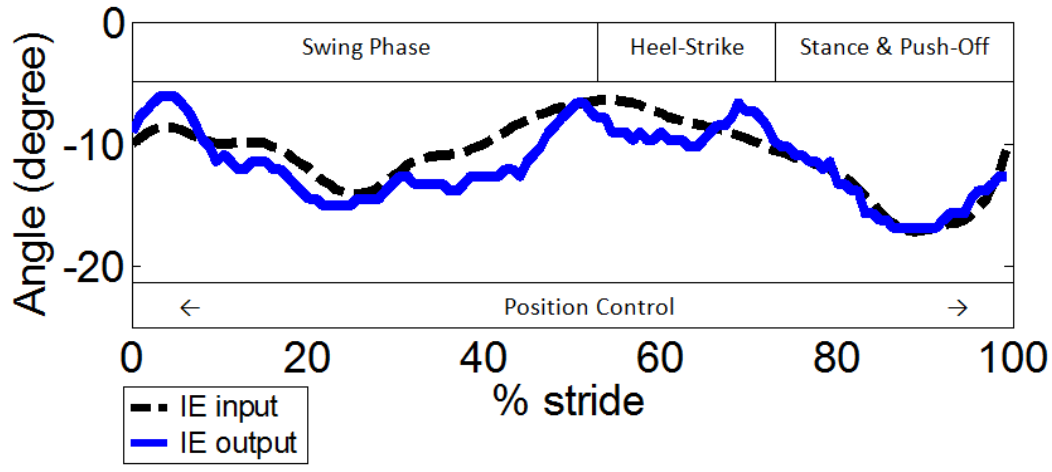

Figure 8.15. Input and output (compensated for 75 milliseconds time delay) of the ankle trajectory in IE during a representative gait cycle with admittance and impedance control (top) and position control (bottom).

The tests with the circular treadmill showed that the impedance/admittance controller were capable of better tracking the desired reference trajectory while decreasing the maximum reaction torques in the foot. Future tests are required to measure the specific contributions of the admittance and impedance controller on the performance improvement, and also how the performance could be improved if only one type of controller was used. In both DP and IE directions, the external torques at both heel strike and push off were greatly reduced at the cost of an extended contact time of the foot with the ground. This characteristic needs to be addressed in our future work with a force feedback gain which enables mimicking the time-varying human impedance during the stance phase. In IE, an increased external inversion torque was developed due to the constraints imposed by the turning disk, and the impedance control was capable of accommodating and 
reducing this external torque. The finite state machine worked as predicted and was capable of properly switching to admittance control at heel strike, and switching back to impedance control at push off. Also, the finite state machine was capable of adjusting to the stride duration by advancing the foot to the heel strike angle and holding that position until heel strike was detected.

\subsection{CONCLUSION}

In this paper, we presented the preliminary steps towards development of a finite state machine for control of a multi-axis ankle-foot prosthesis. The state machine was capable of switching between impedance and admittance control in both Dorsiflexion-Plantarflexion (DP) and Inversion-Eversion (IE). Strain gauges were installed on the prosthesis' foot that were successfully used to estimate the external torques in DP and IE. The estimated torques were used for torque feedback in the impedance and admittance controllers. The quasi-static stiffness of the prosthesis in impedance controller was evaluated, showing a near linear relationship between the torque feedback gain and the quasi-static stiffness of the ankle. This showed that the impedance controller was capable of modulating the stiffness of the ankle in a predictable manner. The finite state machine and controllers were also evaluated with a circular treadmill evaluation platform and the results were compared to the performance of the prosthesis with a position controller and with no control at all. These experiments showed that the impedance/admittance controller with a fixed torque feedback gain of 0.5 for both DP and IE was capable of better tracking the desired reference trajectory compared to the position control and when there was no control, while decreasing the reaction torques at the foot. 


\section{9- DESIGN AND EVALUATION OF A 2-DOF INSTRUMENTED PLATFORM FOR ESTIMATION OF THE ANKLE MECHANICAL IMPEDANCE DURING GAIT IN ARBITRARY DIRECTIONS}

Evandro Ficanha, Guilherme Ribeiro, Mo Rastgaar

\subsection{ABSTRACT}

This paper describes the fabrication and preliminary evaluation of an instrumented vibrating walkway designed for estimation of the human ankle's mechanical impedance in two degrees of freedom (DOF), namely DorsiflexionPlantarflexion (DP) and in Inversion-Eversion (IE) during walking in arbitrary directions. The main component of the system is a 2-DOF vibrating platform. The device consists of two modules, and actuation module and a force plate module. The actuation module uses two voice coil linear motors to generate torque perturbations up to $168 \mathrm{Nm}$ in DP and $26 \mathrm{Nm}$ in IE in the force plate module. The force generated in the motors is transferred to the force plate module using Bowden cables, providing a low-profile system that can be installed in a walkway. In the force plate module, the frame that holds a force plate rotates in two degrees of freedom, applying torque perturbations to the human subjects's ankle in DP and IE directions. The ankle's rotational motion is measured using a motion capture camera system. The analytical and numerical approaches for estimation of the ankle's torque, rotation, and impedance are presented. A system validation experiment using a mockup was conducted to verify the system's ability to estimate the impedance of a physical system in 2 DOF. The results showed that the developed system and protocol was capable of identifying the effects of the inertia, damping, and stiffness of the mockup up to $30 \mathrm{~Hz}$. The estimated mockup's

\footnotetext{
* The material contained in this chapter is in preparation for submission to a journal.
} 
impedance magnitude at low frequencies (2 $\mathrm{Hz}$ ) using a multi-variable stochastic identification method was shown to be within $0.05 \%$ and $1.1 \%$ of the quasi-static stiffness of the mockup in DP and IE, respectively.

\subsection{INTRODUCTION}

The human ankle function is significant during walking and standing. During walking, the ankle supports the body weight, generates propulsion, contributes in stability, while rotating and generating torques in DP, IE and internal externalinternal directions (5). The ankle is also involved, among other functions, in balance and shock absorption. The mechanical impedance of the ankle changes continuously during a stride to accomplish this variety of tasks. The mechanical impedance of a physical system correlates an input motion to the system to the output reactive forces. In this paper the mechanical impedance may be referred simply as impedance. The impedance is a function of the system's inertia, damping, and stiffness, and represents the system's resistance to motion as a function of the frequency of the input motion. For the human ankle, the impedance is defined as the relationship between the angular motion disturbances to the ankle and its output reactive torques. The ankle impedance is both time-varying and task-dependent in the sense that it constantly changes during different phases of gait depending on the gait type $(48,104)$. For example, it has been shown that the variation of the ankle impedance during straight walking changes at different gait speeds; and although has yet to be measured to the best knowledge of the authors, there is strong evidence that the ankle impedance modulation is significantly different when comparing straight walking to turning maneuvers such as step turn or sidestep cutting $(5,6)$. This paper, presents the design and preliminary evaluation results of a platform that allows for estimation and comparison of the mechanical impedance of ankle in DP and IE during different gait scenarios.

The human ankle's capability to generate net positive work during gait and the ankle's quasi-static impedance (stiffness) have been the core concepts in the design 
of powered ankle-foot prostheses. Such approach allows amputees to benefit from prostheses that have similar impedance and mechanical characteristics as the unimpaired human ankle, resulting in a potentially more natural gait with lower risks of secondary injuries. Several powered prostheses that mimic the human ankle's quasi-static impedance have been designed. For example, the powered ankle and knee prosthesis developed by Sup et al. is capable of controlling the mechanical impedance of both the knee and ankle in the sagittal plane $(26,28)$. Hitt et al. developed SPARKy, a tendon driven ankle-foot prosthesis capable of generating a net positive work during walking and running $(68,69)$. Au et al. developed early prototypes of $\mathrm{BiOM} \AA$, an ankle-foot prosthesis which uses a finite state machine to identify the state of gait and adjust the prosthesis to generate the appropriated torques and net positive work during plantarflexion. Although theses prostheses greatly improve the amputees' gait, a better understanding of the timevarying and task-dependent impedance of ankle in both sagittal and frontal planes can further advance the state of the art in lower limb prosthetic devices.

Different studies have explored the 1-DOF ankle impedance in the sagittal plane under no load (73-87); however, this may not be a sufficient representation of the ankle impedance during standing and walking, where the impedance varies during the different phases of each stride. Estimation of the mechanical impedance of ankle in load bearing condition has been studied in the sagittal plane $(20,22,47,48,91$, $92,94,96,105)$ and in the frontal plane $(89,90)$. Single DOF ankle movements rarely happen during normal gait, resulting in unique challenges in the control of ankle-foot prosthesis with multiple DOF (46). This implies that a comprehensive understanding of the time-varying and task-dependent ankle impedance of the human gait during different types of gait such as turning, traversing slopes, and walking up or down slopes can greatly advance the state of the art in lower extremity assistive devices and robots.

The time-varying impedance of the ankle during walking has been explored by two research groups. Rouse et al. developed a perturbation platform with single 
active DOF (49). The platform applied single and isolated perturbations to the ankle in DP at four distinct points of the stance phase of straight walk of unimpaired subjects. The perturbations were applied within $13 \%$ up to $63 \%$ of the duration of the stance phase, showing great variability in the ankles stiffness $(1 \mathrm{Nm} / \mathrm{rad} / \mathrm{kg}$ at 0.1 seconds and $4.6 \mathrm{Nm} / \mathrm{rad} / \mathrm{kg}$ at 0.475 seconds into the stance phase) and damping $(0.012 \mathrm{Nms} / \mathrm{rad} / \mathrm{kg}$ at 0.1 seconds and $0.038 \mathrm{Nm} / \mathrm{rad} / \mathrm{kg}$ at $0.475 \mathrm{~seconds}$ into the stance phase) with an increased ankle stiffness and damping at push-off compared to the heel-strike (48). A different study was conducted by Lee et al. on the time-varying mechanical impedance of ankle in both DP and IE simultaneously (104). In their study, the subjects used a wearable robot that applied continuous perturbations to the human ankle while the subject walked on a treadmill. The information about the ankle properties during the swing phase, early stance, and toeoff was used to estimate the time-varying impedance of the ankle during those phases using a method developed for identification of physiological systems with time-varying dynamics (106). Their protocol did not determined the ankle impedance during the mid-stance phase of the gait; however, it showed great variability of the ankle impedance in both DP and IE directions during different phases of walking on a straight path.

This paper presents the design and preliminary validation of the developed instrumented vibrating platform that is capable of applying torque perturbations to the ankle in two DOF during different types of gait. The developed device expands the functionality of the perturbating platform developed by Rouse et al (49) to estimate the time-varying impedance of the ankle in both DP and IE during gait by applying torque perturbations to the ankle in those two DOF. Additionally, the design aims to allow such study not only during the straight walking, but also during turning in arbitrary directions. For this reason, the design has a low profile suitable for being installed into a walkway. In this platform, the human subjects do not carry any wearable device that might change the dynamic of the gait. The developed device only require the placement of infrared markers on the subject's body for 
tracking the ankle trajectories and rotations. Additionally, the participants are not constrained to walk on a treadmill or in straight path.

This paper first describes in detail the design and fabrication of the instrumented vibrating platform. Next, the developed system was evaluated using an ankle-foot mockup to demonstrate the capability of the system and the developed protocols in identifying the mechanical impedance of a physical system. The mathematical tools to calculate the ankle torques and angles based on the information obtained from the force plate and camera system are explained. Additionally, the stochastic identification method that was used for impedance estimation of the ankle-foot mockup based on the ankle angles and torques is presented. The paper describes the hardware and mathematical tools to develop a system for future estimation of the time-varying and task-dependent impedance of the human ankle in two DOF.

\subsection{INSTRUMENTED VIBRATING PLATFORM DESIGN}

The developed vibrating platform (Fig. 9.1) consists of two independent modules; actuation module and the force plate module. The actuation module contains all the power electronics including servo drivers and voice coil linear motors. The force plate module consists of a force plate mounted on a tilting mechanism allowing the force plate to rotate in two DOF about the longitudinal and lateral axes of the force plate. The force plate module was design to be easily installed in walkways for gait analysis and has a compact height of $0.159 \mathrm{~m}$. This low profile is achieved by separating the actuation and the force plate modules. The power from the actuation module is transferred to the force plate module by four Bowden cables generating torque perturbations. 


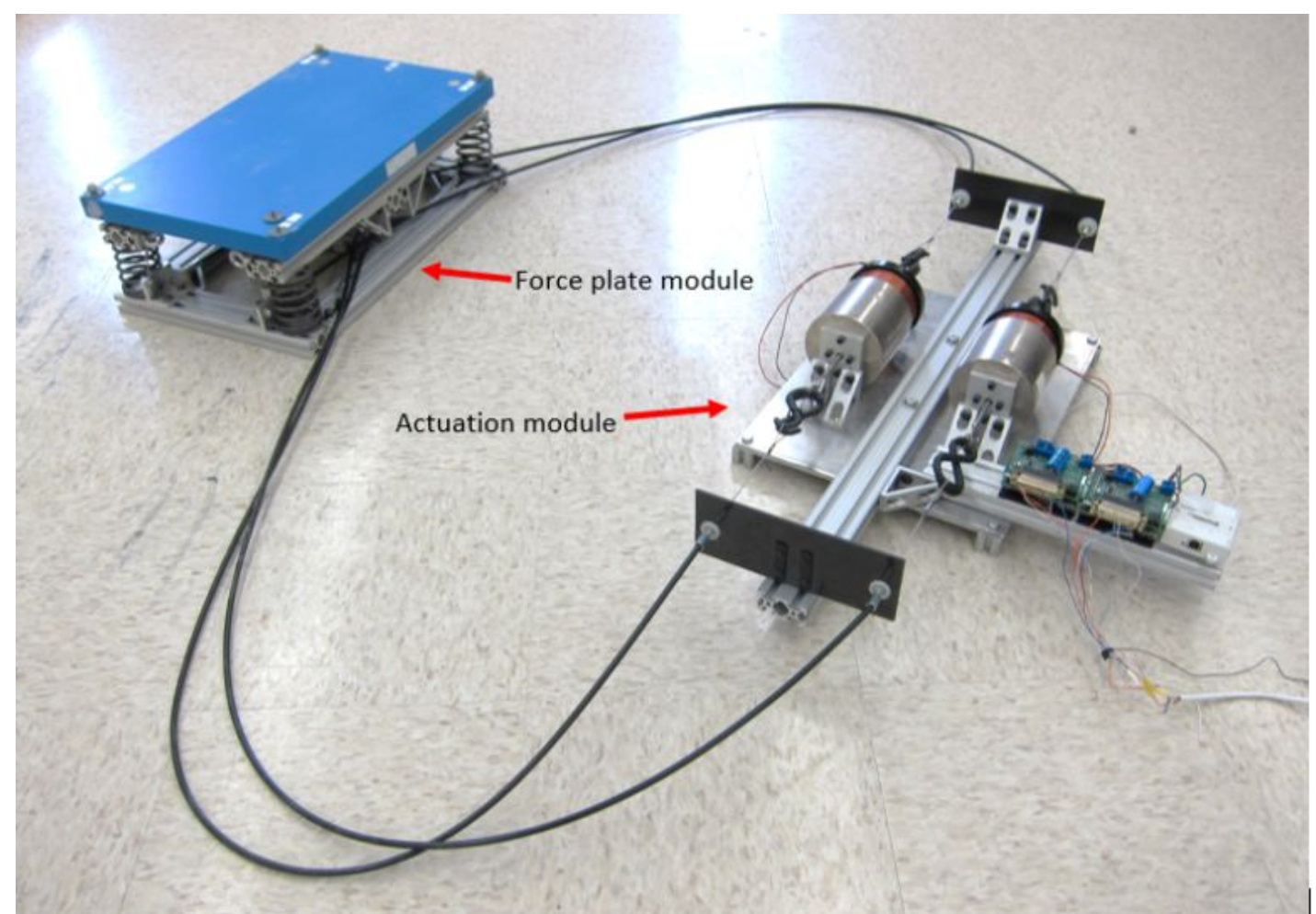

Figure 9.1. Instrumented vibrating platform consists of the actuation and force plate modules.

\subsubsection{ACTUATION MODULE DESIGN}

The actuation module (Fig. 9.2) is driven by two voice coil linear motors (C) (Moticont $^{\circledR}$ GVCM-095-089-01). The motors are rated at $351 \mathrm{~N}$ of force at $10 \%$ duty cycle and $111 \mathrm{~N}$ continuous force. They have a $63.5 \mathrm{~mm}$ stroke length, a coil resistance of $3 \mathrm{Ohms}$, and coil inductance of $2.1 \mathrm{mH}$ at $1000 \mathrm{~Hz}$. The voice coils motors are powered by a pair of digital servo drivers (D) (Moticont ${ }^{\circledR}$ 510-03) capable of delivering up to $40 \mathrm{~A}$ at $20 \mathrm{kHz}$ PWM frequency. The servo drivers are connected to two deep cycle batteries providing $24 \mathrm{~V}$ and 114 ampere-hours capacity. The motors run with open-loop current control and receive analog control signals from a DAC (E) (National Instrument ${ }^{\circledR}$ NI USB 6009) connected to a computer. Each motor is connected to two ultra-flexible steel cables (F) with 1.6 mm diameter (McMaster-Carr part number 3423T29). The cables are coated with fluorinated ethylene propylene for its nonstick properties helping to reduce drag and 
wear in the Bowden cables. Two cables are attached to each end of each motor; therefore, each motor can pull on one cable while releasing the tension on the cable at the opposite side. The Bowden cable housings are attached to flexible carbon fiber springs (G) (McMaster-Carr part number 8194K56) that are preloaded to assure the cables are always under tension. The flexible carbon fiber springs are mounted to a rail $(\mathrm{H})$, allowing the springs to slide and lock in the same axis as the tension of the cables. This allows for easy adjustment of the position of the motors with respect to the force plate so the force plate becomes horizontal at the mid stroke of the motors. Additionally, the rail allows for preloading of the tension of the steel cables. Fine adjustment of the cable tension and centering the motors (to the mid stroke of the motors) to level the force plate can be obtained by adjusting the Bowden cable housing adjusters (I) (Parts Reloaded part number 935) located at the carbon fiber springs. Adequate pre-load in the cables is important as excessive cable tension increases friction inside the Bowden cables, increasing wear and decreasing the available power to the force plate module. Insufficient cable pre-load results in cable slack during operation. For optimum performance, before performing experiments, the tension in the cables should be adjusted and calibrated accordingly before the experiments.

The voice coil motors generate large magnetic fields when operating which induce a voltage on the motor's supporting frame, steel cables, and rail. This voltage, if not insulated from the force plate, generates excessive noise in the force plate readings. To avoid this voltage from reaching the force plate module through the steel cable and the Bowden cable housing, electrical insulators were installed. Two nylon " $\mathrm{S}$ " hooks $(\mathrm{K})$ rated at $530 \mathrm{~N}$ of work load were attached in line with each cable to electrically insulate the cables from the actuation module. The Bowden cable housing, which are made of steel wrapped in plastics, were electrically insulated using nylon sleeves and washer $(\mathrm{J})$, where the cable housing adjusters (I) connect to the carbon fiber springs $(\mathrm{G})$. 


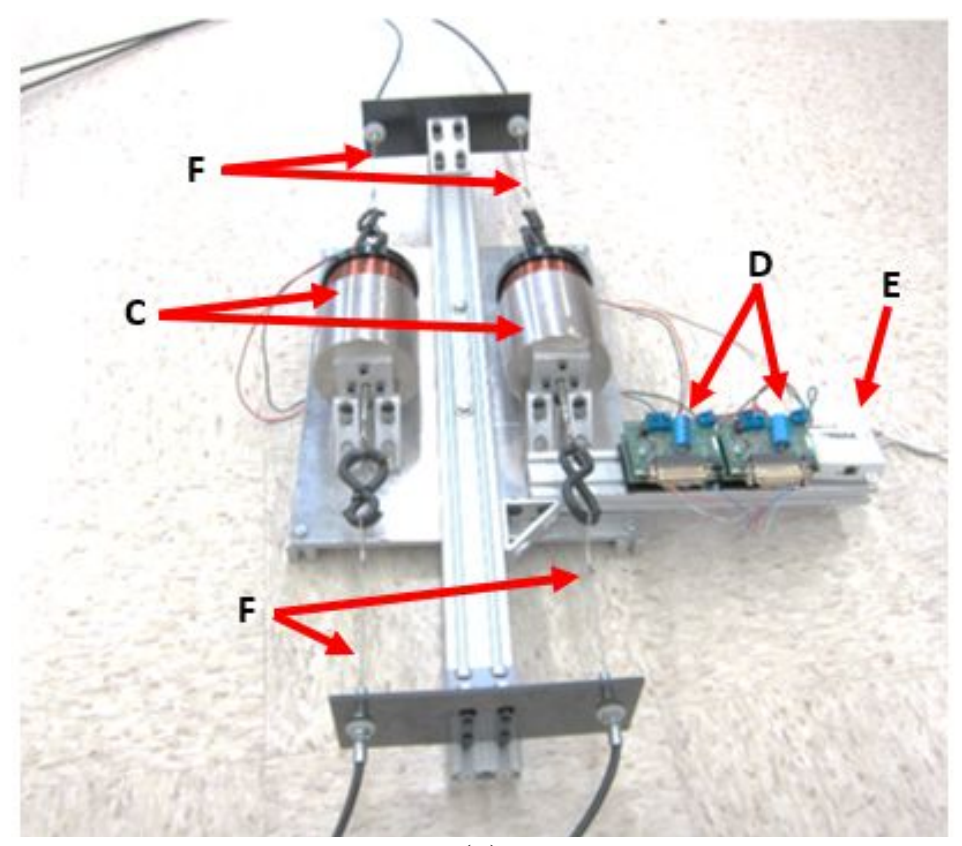

(a)

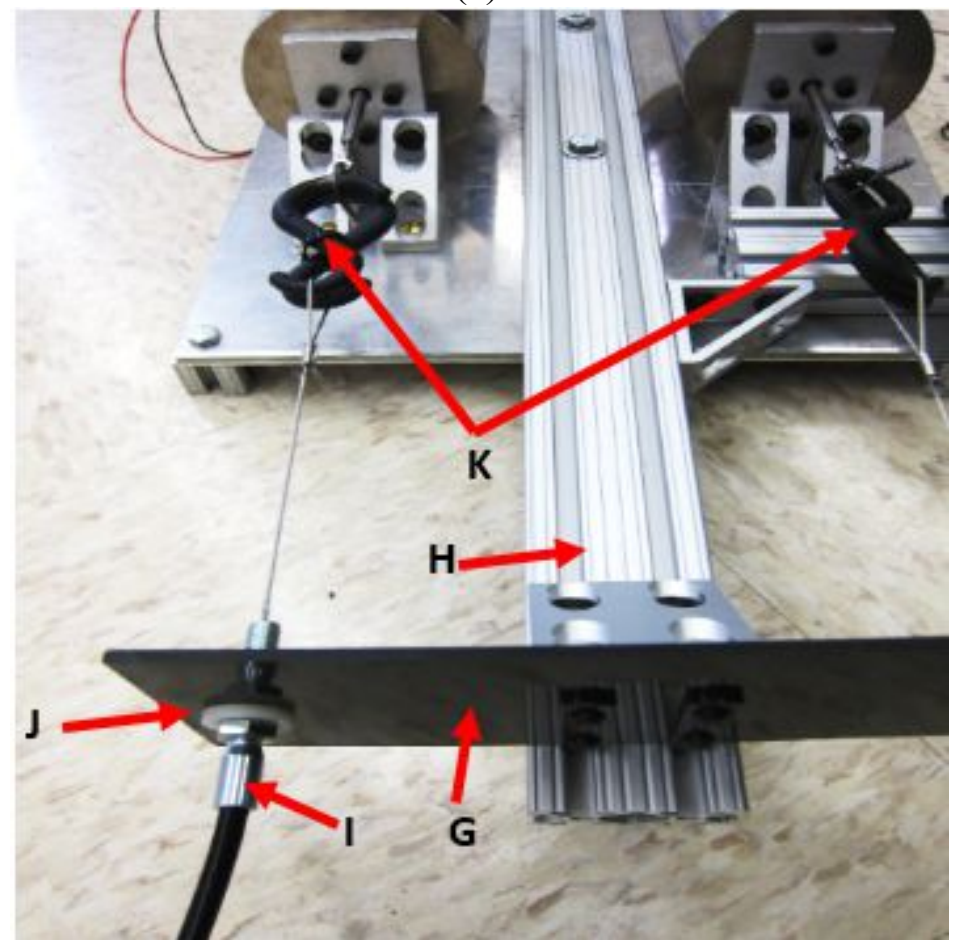

(b)

Figure 9.2. Actuation module and its components. (a) Actuation module main components: (C) voice coil motors, (D) servo drivers, (E) analog to digital converter, (F) steel cables. (b) Detail of actuation module components: (G) carbon fiber spring, (H) sliding rail, (I) Bowden cable housing adjusters, $(\mathrm{J})$ nylon washers, (K) nylon "S" hooks. 


\subsubsection{FORCE PLATE MODULE}

Fig. 9.3 show the force plate module with and without the force plate (L) (Kistler ${ }^{\circledR}$ 9260AA3) coupled to the upper frame (Q), respectively. This versatile design allows the force plate to be easily attached to and detached from the upper frame so it can be used in other applications. The force plate feet (M) are inserted into sleeves $(\mathrm{N})$ located at the upper frame. A tight fit between the feet and the sleeves are assured by rubber O-rings around each foot, assuring the force plate is constrained in the horizontal plane with respect to the upper frame. Neodymium magnets (McMaster-Carr part number 5679K15) are used at the bottom of each sleeve and are rated at $196 \mathrm{~N}$ of puling force. The magnets are bolted to the upper frame and hold the force plate feet inside the sleeves holding the force plate to the frame and constrain it from moving in the vertical axis during the device operation. This approach facilitates insertion and removal of the force plate into the upper frame, while constraining any relative motion between the two components without the need of any permanent modification of the force plate.

At the center of the upper frame $(\mathrm{Q})$ and at the center of the lower frame $(\mathrm{R}) \mathrm{a}$ universal joint $(\mathrm{O})$ constrains both frames from any relative translational motion and rotational motion about the vertical axis. Additionally, it supports the user's weight during the experiments. To reduce friction and increase load bearing capacity, a compact universal joint with low friction needle bearings was used (McMaster-Carr part number $8284 \mathrm{~K} 73$ ). The rotation of the universal joint corresponds to the relative motion of the upper frame (constrained to the force plate) and the lower frame (constrained to the ground) imposing a motion to the ankle equivalent to DP and IE when a subject's foot is on top of the force plate. Springs (P) maintain the force plate centered in a level position. During the experiments, the human subject and the motors will generate torques at the force plate. The motors run in open loop current control with no position control while the person's ankle will generate the 


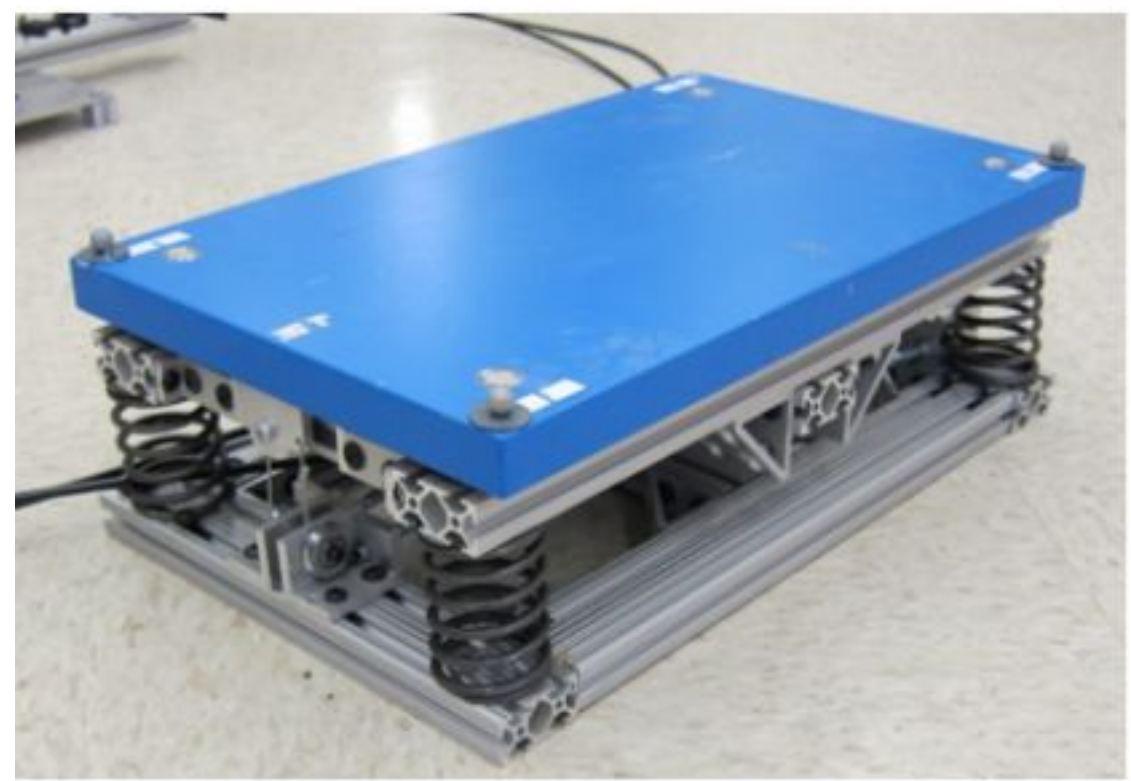

(a)

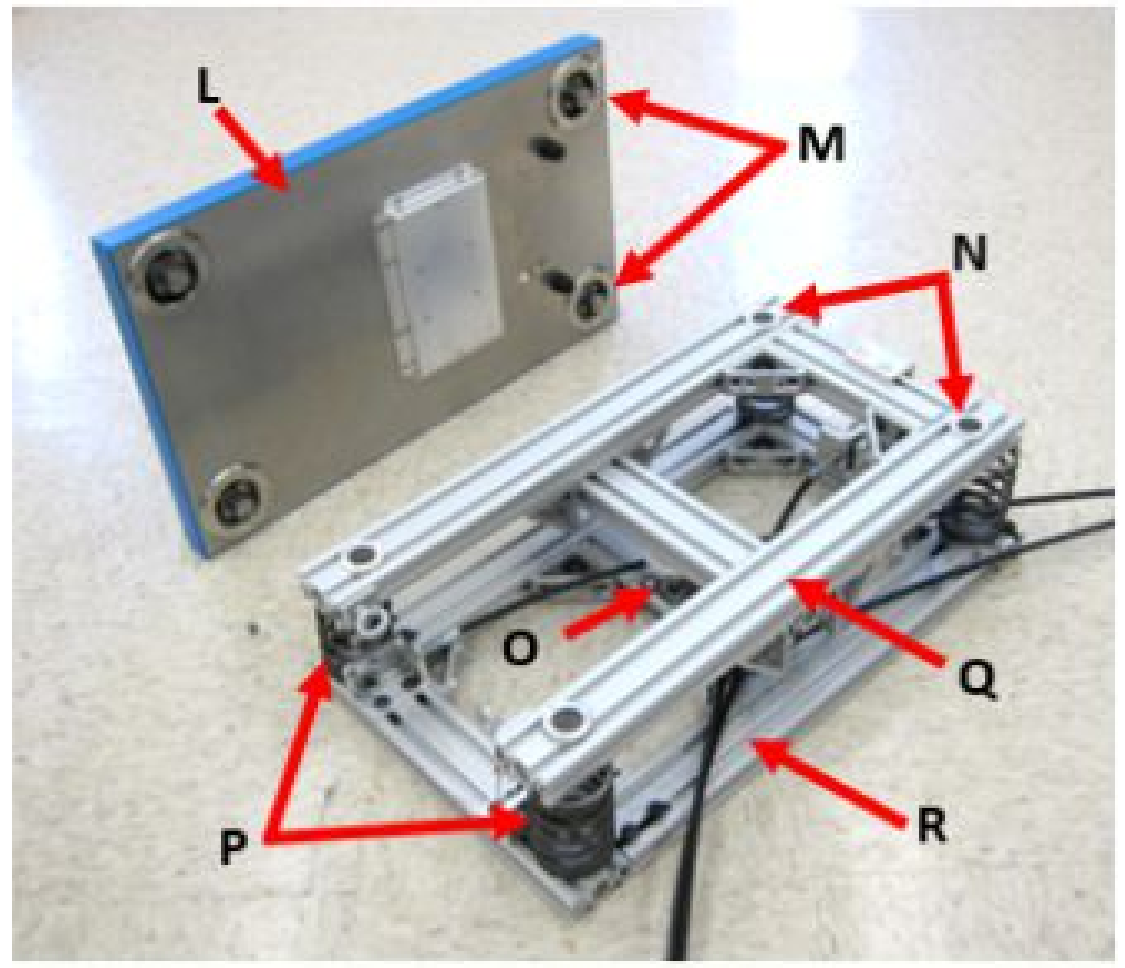

(b)

Figure 9.3. Force plate module. (a) Force plate module with the force plate attached to the upper frame and infrared markers attached to its three corners. (b) Force plate module with the force plate removed to show the internal components: (L) force plate, $(\mathrm{M})$ force plate feet, $(\mathrm{N})$ sleeves on the upper frame to insert the force plate feet, $(\mathrm{O})$ universal joint, $(\mathrm{P})$ springs, $(\mathrm{Q})$ upper frame, $(\mathrm{R})$ lower frame. 
torques required for walking. Both of these torques can cause the force plate to move from its central position. The springs are responsible for limiting the force plate rotations due to these torques and returning the force plate to its central position when these forces are removed. In other words, the springs counter the torque generated by the human subjects, while the motors apply perturbation torques to the force plate and the human subjects' ankle simultaneously.

The housing ends of four Bowden cables (T) are attached to the lower frame as shown in Fig. 9.4. The cables rotate upwards at the pulleys (U) and are connected to the upper frame at three points (S and V). Rotating the cables upwards using pulleys allows the mounting the Bowden cables to the upper frame vertically while avoiding a small radius of turn in the Bowden cables. This allows for a short overall height of the force plate module, decreased friction, and decreased wear in the Bowden cables. As shown in Fig. 9.4(b), at the point of connection (V) where the steel cables connect to the upper frame, a pulley is installed. The cable comes from one Bowden cable housing, turns upwards $90^{\circ}$ at the first pulley (U), turns $180^{\circ}$ downwards at the pulley (V), and turns $90^{\circ}$ at the second pulley (U) back to another Bowden cable housing. On the other side of the force plate (Fig. 9.4(a)), the steel cables come out of one of the Bowden cable housings, turn upwards $90^{\circ}$ at the pulleys (U), and connect to the upper frame at one of the points (S). There are three segments of cable, which two of them are mounted in a similar way. In those two similar segments, one end of the cables is attached to a motor and the other end is attached to one of the points $(\mathrm{S})$ in the upper frame. The third cable segment is twice as long as the other two cable segments and its both ends are attached to the two motors at the same side of the actuation module. The midpoint of this cable, when the force plate is at its central position, is at the pulley $(\mathrm{V})$. The points $(\mathrm{V})$ and $(\mathrm{S})$ are at 0.24 $\mathrm{m}\left(L_{X}\right)$ from the universal joint in the longitudinal axis of the force plate module, but in the opposite sides of the upper frame. These lengths are the moment arms for DP torque generation. The distance between the points $(\mathrm{S})$ to the universal joint are $0.075 \mathrm{~m}$ 


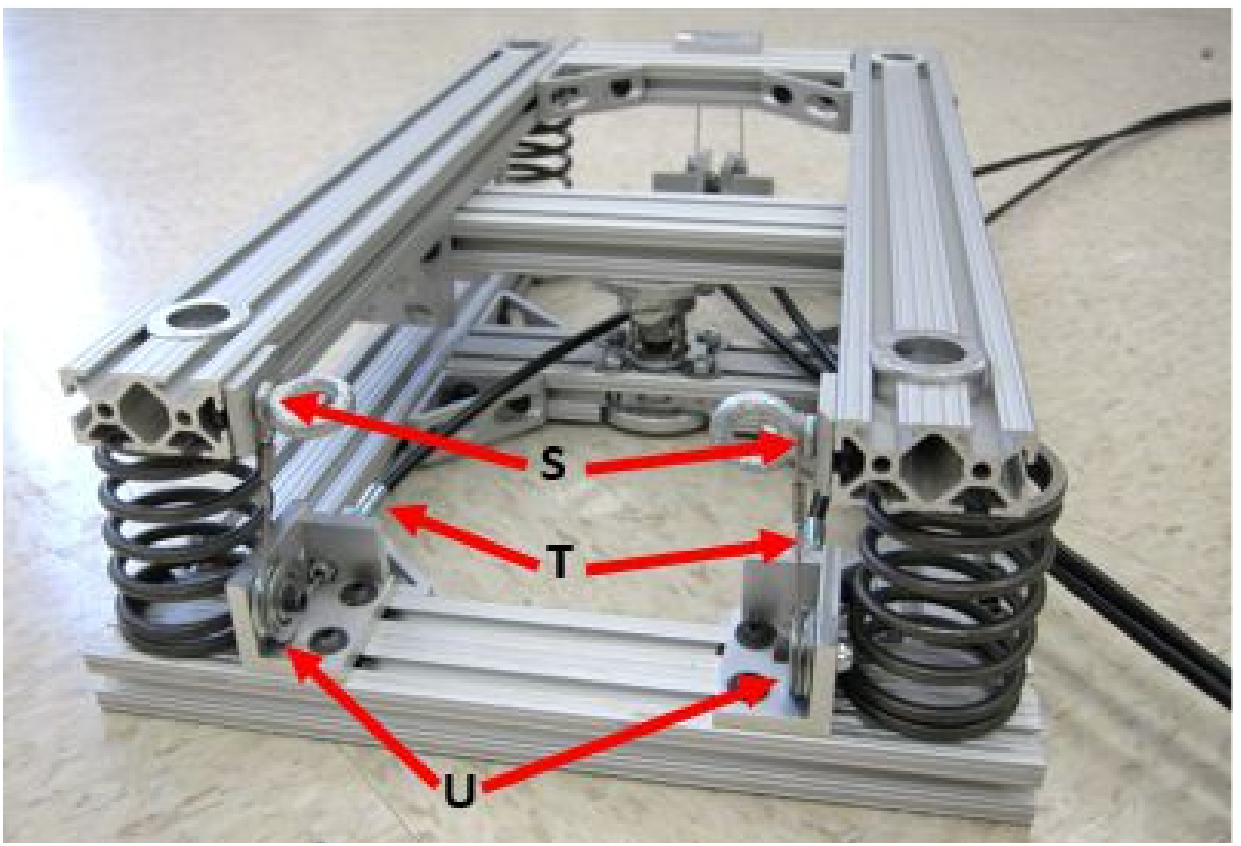

(a)

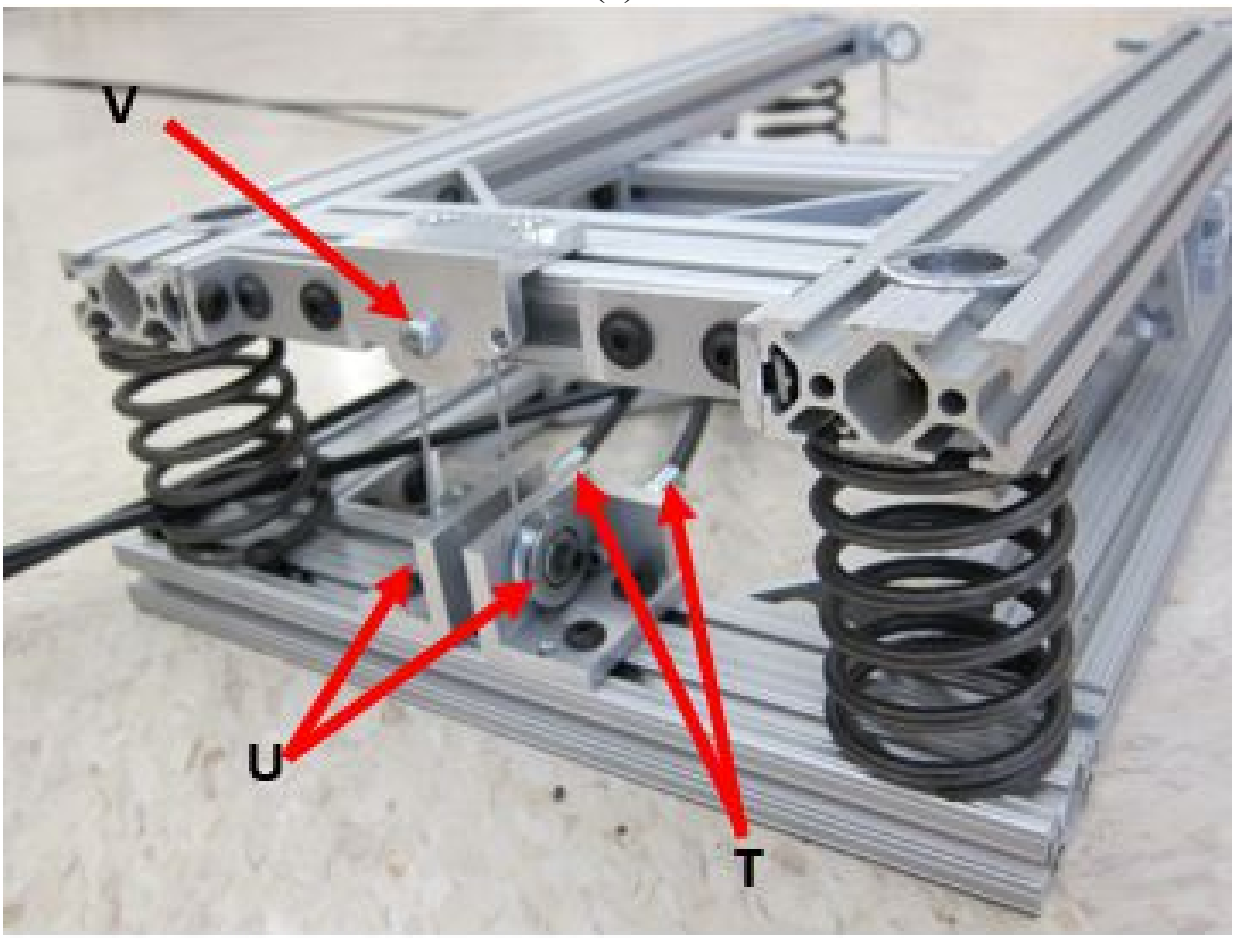

(b)

Figure 9.4. Detailed view of the force plate module. (a) Front view of the force plate module: (T) Bowden cable housing stopper, $(\mathrm{U})$ pulley, $(\mathrm{S})$ point of connection of the cable to the top frame. (b) Rear view of the force plate module: (T) Bowden cable housing stopper, $(\mathrm{U})$ pulley, $(\mathrm{V})$ point of connection of the steel cable to the top frame by a pulley (pulley not visible). 
$\left(L_{Y}\right)$ in the lateral axis and are the moment arms for the IE torque generation. Symmetrical moment arms are important otherwise the cable length would change and become slack as the upper frame rotates away from the central position. However, some change in cable length is still observed. Linear motion of the cables generates rotational motion in the upper frame. This effectively changes the cables' lengths as the upper frame deviates away from is central position as the motion of the points $(\mathrm{V})$ and $(\mathrm{S})$ are not linear with respect to the lower frame. However, during the experiments, the angular displacement of the upper frame is small $\left(<2^{\circ}\right)$, resulting in small changes in cable length. The pre-loading of the carbon fiber springs $(\mathrm{G})$ in the actuation module can easily accommodate for the changes in the cables' lengths; therefore, the cables never become slack.

Initial estimation of the platform requirements showed that the platform's stiffness should be large enough to result in its minimal angular displacement due to the user input torque. This is important to minimize variations in gait pattern. However, the platform needs to be compliant enough to allow the motors to rotate it. The actuation system should generate torques larger than the maximum torque expected from a human's ankle to be able to generate perturbation torques to the ankle at all times. The ankle torque for a person weighing $75 \mathrm{~kg}$ during walking reaches up to $140 \mathrm{Nm}$ in DP (107). The mechanism in the force plate module uses the addition and differences of the pulling forces of the motors to generate DP and IE torques, respectively. The upper frame was design with moment arms $\left(L_{X}\right)$ of $0.24 \mathrm{~m}$ in DP and $0.075 \mathrm{~m}$ in IE $\left(L_{Y}\right)$, and the motors (maximum pulling force of $351 \mathrm{~N}$ each) generate a combined maximum torque of $168 \mathrm{Nm}$ in DP and $26 \mathrm{Nm}$ in IE. The current setup of the platform uses four springs $(\mathrm{P})$ rated at $12 \mathrm{kN} / \mathrm{m}$ and are attached at each corner of the force plate module between the upper and lower frames. The springs are preloaded 0.025 meters and generate a rotational stiffness of $10.7 \mathrm{kNm} / \mathrm{rad}$ in DP and $7.2 \mathrm{kNm} / \mathrm{rad}$ in IE. The platform stiffness can be adjusted to different users' or experiments' requirements by moving the springs closer or farther away from the universal joint. Moving the springs closer or farther away 
from the universal joint in the longitudinal axis would decrease or increase the stiffness in DP, respectively. Similarly, moving the springs closer or farther away from the universal joint in the lateral axis would decrease or increase the stiffness in IE, respectively. Also, different spring stiffness or a combination of springs can be used to generate the desired stiffness of the platform.

A side view diagram of the force plate module with a foot on the force plate is shown in Fig. 9.5. The force plate coordinate system (XYZ) was defined with the $X$ axis pointing forward from the human subject point of view, and the $\mathrm{Z}$ axis pointing upwards. The force plate coordinate system is attached to the force plate, and can be described in the global coordinate system $\left(\mathrm{X}_{0} \mathrm{Y}_{0} \mathrm{Z}_{0}\right)$ using a rotation matrix obtained from the camera system recorded data. The net torque about the universal joint in the $\mathrm{Y}$ axis at the upper frame of the force plate module $\left(\tau_{D P_{-} F P}\right)$ can be calculated by balancing the torques applied to the upper frame about the Y axis:

$$
\begin{aligned}
\tau_{D P_{-} F P}=J_{Y} \ddot{\theta}_{D P} & =-\left(F_{V}-F_{S 1}-F_{S 2}\right) L_{\mathrm{X}}+\left(K_{(1+2)}+K_{(2+3)}\right) L_{X} \theta_{D P} \\
& +\left(F_{Z(1+4)}-F_{Z(2+3)}\right) L_{X}+\left(F_{X(1+4)}+F_{X(2+3)}\right) L_{Z}
\end{aligned}
$$

where $J_{Y}$ is the mass moment of inertia of the upper frame about the $\mathrm{Y}$ axis. $F_{V}, F_{S 1}$, and $F_{S 2}$ represent the motors force at the points $\mathrm{V}$ and $\mathrm{S}$ (Fig. 9.4). The moment arm $L_{X}$ is the constant distance (assuming small angles) from the universal joint to the points $\mathrm{V}$ and $\mathrm{S}$ in the $\mathrm{X}$ axis of the upper frame. With similar assumption, the moment arm $L_{Z}$ is the constant distance from the universal joint to the points $\mathrm{V}$ and $\mathrm{S}$ in the $\mathrm{Z}$ axis of the upper frame. Each corner of the force plate module has one spring, $K_{(1+2)}$ and $K_{(3+4)}$ represent the sum of the spring constants of the two springs at each side of the force plate module in the $\mathrm{X}$ axis. $\theta_{D P}$ is the rotation of the upper frame about the $\mathrm{Y}$ axis measured with a motion capture camera system. Additionally, each corner of the force plate has one tri-axial force sensor. $F_{\mathrm{Z}(1+2)}$ and $F_{\mathrm{Z}(3+4)}$ represent the sum of the forces in the $\mathrm{Z}$ axis and $F_{\mathrm{X}(1+2)}$ and $F_{\mathrm{X}(3+4)}$ represent the sum of the forces in the $\mathrm{X}$ axis measured from the two force sensors at each side of the force plate module in the $\mathrm{X}$ axis. 


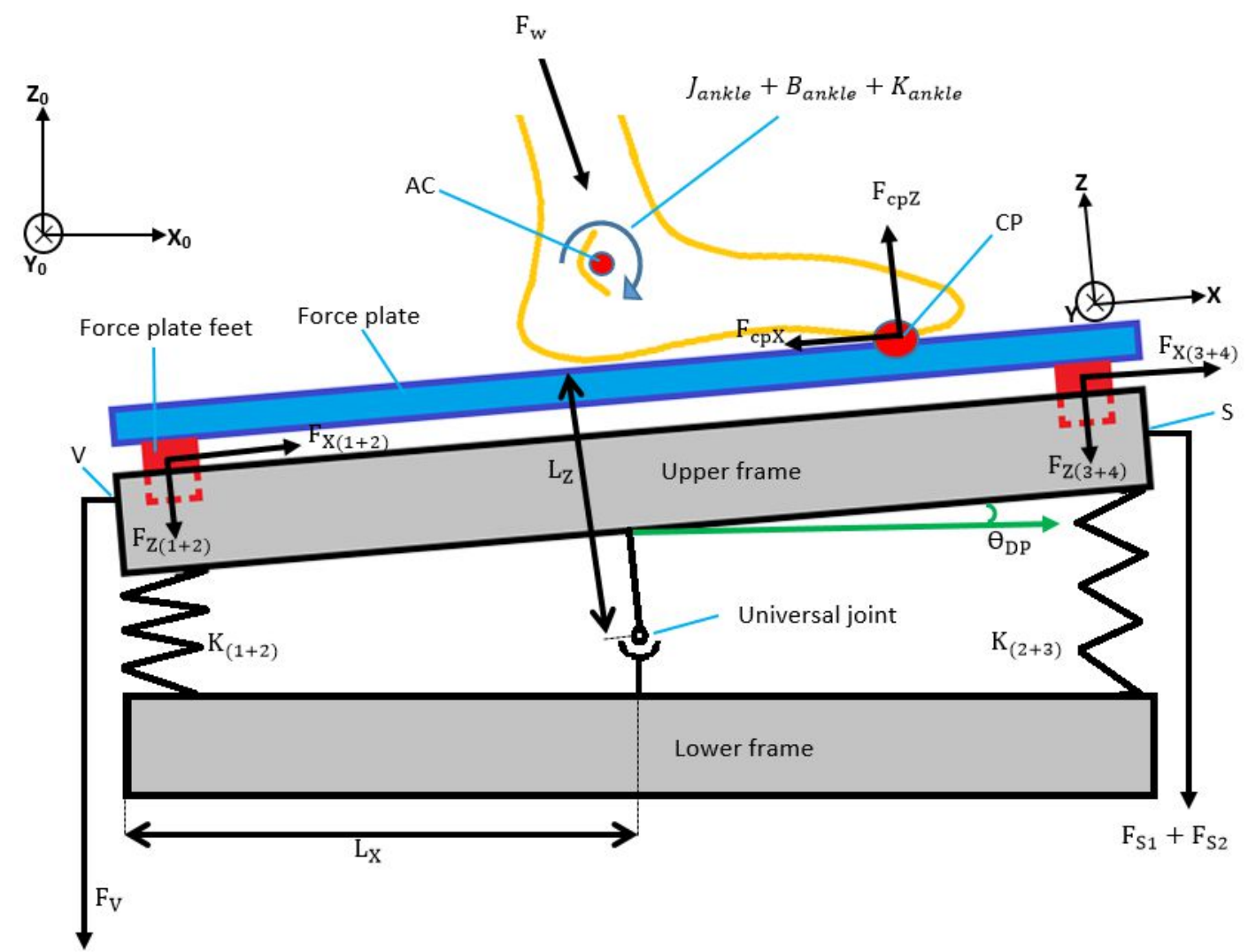

Figure 9.5. Side view sketch of the force plate module with a foot on the force plate. $\mathrm{F}_{\mathrm{w}}$ is the normal force at the ankle joint from the subject's body weight. $\mathrm{F}_{\mathrm{V}}, \mathrm{F}_{\mathrm{S} 1}$, and $\mathrm{F}_{\mathrm{S} 2}$ are the motors' force at the point $\mathrm{V}$ and at the two points $\mathrm{S}$ (Fig. 9.4). The moment arm $L_{X}$ is the distance from the universal joint to the points $\mathrm{V}$ and $\mathrm{S}$ along the $\mathrm{X}$ axis of the upper frame. The moment arm $\mathrm{L}_{\mathrm{Z}}$ is the distance from the universal joint to the points $\mathrm{V}$ and $\mathrm{S}$ along the $\mathrm{Z}$ axis of the upper frame. $\mathrm{K}_{(1+2)}$ and $\mathrm{K}_{(3+4)}$ represent the sum of the spring constants of the two springs on each side of the force plate module in the $\mathrm{X}$ axis. $\Theta_{\mathrm{DP}}$ is the rotation of the uppe $\mathrm{r}$ frame about the $\mathrm{Y}$ axis measured with a motion capture camera system. $\mathrm{F}_{\mathrm{Z}(1+2)}$ and $\mathrm{F}_{\mathrm{Z}(3+4)}$ represent the sum of the forces in the $\mathrm{Z}$ axis and $\mathrm{F}_{\mathrm{X}(1+2)}$ and $\mathrm{F}_{\mathrm{X}(3+4)}$ represent the sum of the forces in the $\mathrm{X}$ axis measured from the two force sensors in each side of the force plate module in the $\mathrm{X}$ axis. $J_{\text {ankle }}, B_{\text {ankle }}$, and $K_{\text {ankle }}$ are vectors containing the ankle's mass moment of inertia, the ankle's rotational damping, and the ankle's rotational stiffness in DP and IE, respectively. $\mathrm{CP}$ is the center of pressure of the foot on top of the force plate and is calculated from the force plate readings in the force plate coordinate system as described in (5). AC is the ankle center of rotation in the force plate coordinate system that is calculated based on the position and rotation of the foot and shin. 
Similarly, the net torque about the $\mathrm{X}$ axis of the universal joint at the upper frame of the force plate module $\left(\tau_{I E_{-} F P}\right)$ can be calculated by balancing the torques about the $\mathrm{X}$ axis of the upper frame, except only the motors forces applied at the points S (Fig. 9.4) contribute to torque generation in IE since the force applied at the point $\mathrm{V}$ is aligned with the $\mathrm{X}$ axis, thus the moment arm in IE for that force is zero:

$$
\begin{array}{r}
\tau_{I E_{-} F P}=J_{X} \ddot{\theta}_{I E}=-\left(F_{S 1}-F_{S 2}\right) L_{X}+\left(K_{(1+4)}+K_{(2+3)}\right) L_{Y} \theta_{I E} \\
+\left(F_{Z(1+4)}-F_{Z(2+3)}\right) L_{Y}+\left(F_{Y(1+4)}+F_{Y(2+3)}\right) L_{Z}
\end{array}
$$

where $J_{X}$ is the mass moment of inertia of the upper frame about the $\mathrm{X}$ axis. The moment arm $L_{Y}$ is the constant distance (assuming small angles) from the universal joint to the points $\mathrm{S}$ along the $\mathrm{Y}$ axis of the upper frame. $K_{(1+4)}$ and $K_{(2+3)}$ represent the sum of the springs constants of the two springs on each side of the force plate module in the $\mathrm{Y}$ axis. $\theta_{I E}$ is the rotation of the upper frame measured with a motion capture camera system about the $\mathrm{X}$ axis. $F_{\mathrm{Z}(1+4)}$ and $F_{\mathrm{Z}(2+3)}$ represent the sum of the forces in the $\mathrm{Z}$ axis and $F_{\mathrm{Y}(1+4)}$ and $F_{\mathrm{Y}(2+3)}$ represent the sum of the forces in the $\mathrm{Y}$ axis measured from both the force sensors in each side of the force plate module in that direction.

In equations 1 and 2, the measured values are the force values from the force plate and the angles measured by the motion capture camera system. Solving for $\theta_{D P}$ and $\theta_{I E}$ in equations 1 and 2 , and setting $\ddot{\theta}_{D P}$ and $\ddot{\theta}_{I E}$ to zero (static condition), the maximum rotations based on the stiffness of the platform and the maximum force from the motors are determined as $\pm 0.9^{\circ}$ in DP and $\pm 0.3^{\circ}$ in IE. The maximum expected torque is obtained when the motors and the human subject apply a torque in the same direction, which in DP adds up to $308 \mathrm{Nm}$. This torque would cause an upper frame rotation of $\pm 1.8^{\circ}$ that is in the linear range of operation of the force plate module.

To calculate the ankle torques in DP and IE $\left(\tau_{D P}\right.$ and $\left.\tau_{I E}\right)$ the following equations can be derived: 


$$
\begin{aligned}
\tau_{D P} & =\left(J_{D P_{-} \text {ankle }}+J_{D P_{-} \text {plate }}\right) \ddot{\theta}_{D P}+B_{D P_{-} \text {ankle }} \dot{\theta}_{D P}+K_{D P_{-} \text {ankle }} \theta_{D P} \\
& =\left(F_{Z(1+2)}+F_{Z(3+4)}\right)\left(C P_{X}-A C_{X}\right)+\left(F_{X(1+2)}+F_{X(3+4)}\right)\left(C P_{Z}-A C_{Z}\right) \\
\tau_{I E} & =\left(J_{I E_{-} \text {ankle }}+J_{I E_{-} \text {plate }}\right) \ddot{\theta}_{I E}+B_{I E_{-} \text {ankle }} \dot{\theta}_{I E}+K_{I E_{-} \text {ankle }} \theta_{I E} \\
= & \left(F_{Z(1+4)}+F_{Z(2+3)}\right)\left(C P_{Y}-A C_{Y}\right)+\left(F_{Y(1+4)}+F_{Y(2+3)}\right)\left(C P_{Z}-A C_{Z}\right)
\end{aligned}
$$

where $J_{D P_{-} \text {ankle }}, B_{D P_{-} \text {ankle }}$, and $K_{D P_{-} \text {ankle }}$ are the ankle's mass moment of inertia, the ankle's rotational damping, and the ankle's rotational stiffness in DP, respectively. $J_{I E_{-} a n k l e}, B_{I E_{-} \text {ankle }}$, and $K_{I E_{-} \text {ankle }}$ are the ankle's mass moment of inertia, the ankle's rotational damping, and the ankle's rotational stiffness in IE, respectively. Also, $J_{D P \_}$plate and $J_{I E_{-} p l a t e}$ are the force plate's mass moment of inertia in DP and IE, respectively. $C P_{X}, C P_{Y}$, and $C P_{Z}$ are the center of pressure of the foot on top of the force plate in the $\mathrm{X}, \mathrm{Y}$, and $\mathrm{Z}$ axes, respectively as described in (5). $A C_{X}, A C_{Y}$, and $A C_{Z}$ are the ankle center of rotation in the $\mathrm{X}, \mathrm{Y}$, and $\mathrm{Z}$ axes, respectively that are calculated based on the position and rotations of the foot and shin and will be explored later in this paper.

It is important to note that the frame springs $(\mathrm{P})$ and the forces applied by the motors do not affect the ankle torque readings in equations 3 and 4 . That is because the forces transferred by these components are applied at the upper frame (Q) which rotates about the universal joint $(\mathrm{O})$, and are not transferred to the force plate censors. The ankle torque calculation requires the force plate outputs that provide the force values and the center of pressure and the ankle center of rotation. The ankle angles only require the relative rotations of the foot with respect to the shin obtained from the motion capture system. The calculated ankle angles and torques are the combination of the effects of the ankle's impedance and the inertia of the force plate when the foot is on top of the force plate since both share the same motion. To estimate the impedance of the ankle during gait when the motion of the foot and force plate is not the same, it is necessary to transform the force plate readings to the foot coordinate system using a rotational matrix that is defined based on the relative rotations of the foot and the force plate. 


\subsubsection{INSTRUMENTED WALKWAY}

The Instrumented vibrating platform was used in a walkway as shown in Fig. 9.6. The walkway allows the placement of the force plate module so the top surface of the force plate is flush with the walking surface of the walkway. This allows for human subjects to easily stand or walk on the force plate module. The force plate module records the forces applied to the ankle while the motors apply perturbations and the motion of both the force plate and the ankle are recorded using a motion capture camera system. The walkway is $0.159 \mathrm{~m}$ tall (the same as the force plate module), $6 \mathrm{~m}$ long and $1.83 \mathrm{~m}$ wide. It is constructed of solid wood to avoid undesired motion that a hollow walkway could produce. The surface is covered with plywood to provide a level and safe ground. The walkway is modular, in the sense that its length and shape can be configured to different types of turning maneuvers, walking on uneven terrain, or slopes. Also, the force plate module can be replaced by a conventional force plate for standard gait analysis experiments. Reflective markers are mounted in the objects of interest and the camera system emits infrared light which is reflected back to the camera sensors, allowing for the calculation of the position of each marker and subsequent calculation of the position and rotations of each object. The camera system consists of eight Prime $17 \mathrm{~W}$ Optitrack ${ }^{\circledR}$ mounted in a square formation covering a volume of nearly 16 cubic meters and an area of 12 square meters. 


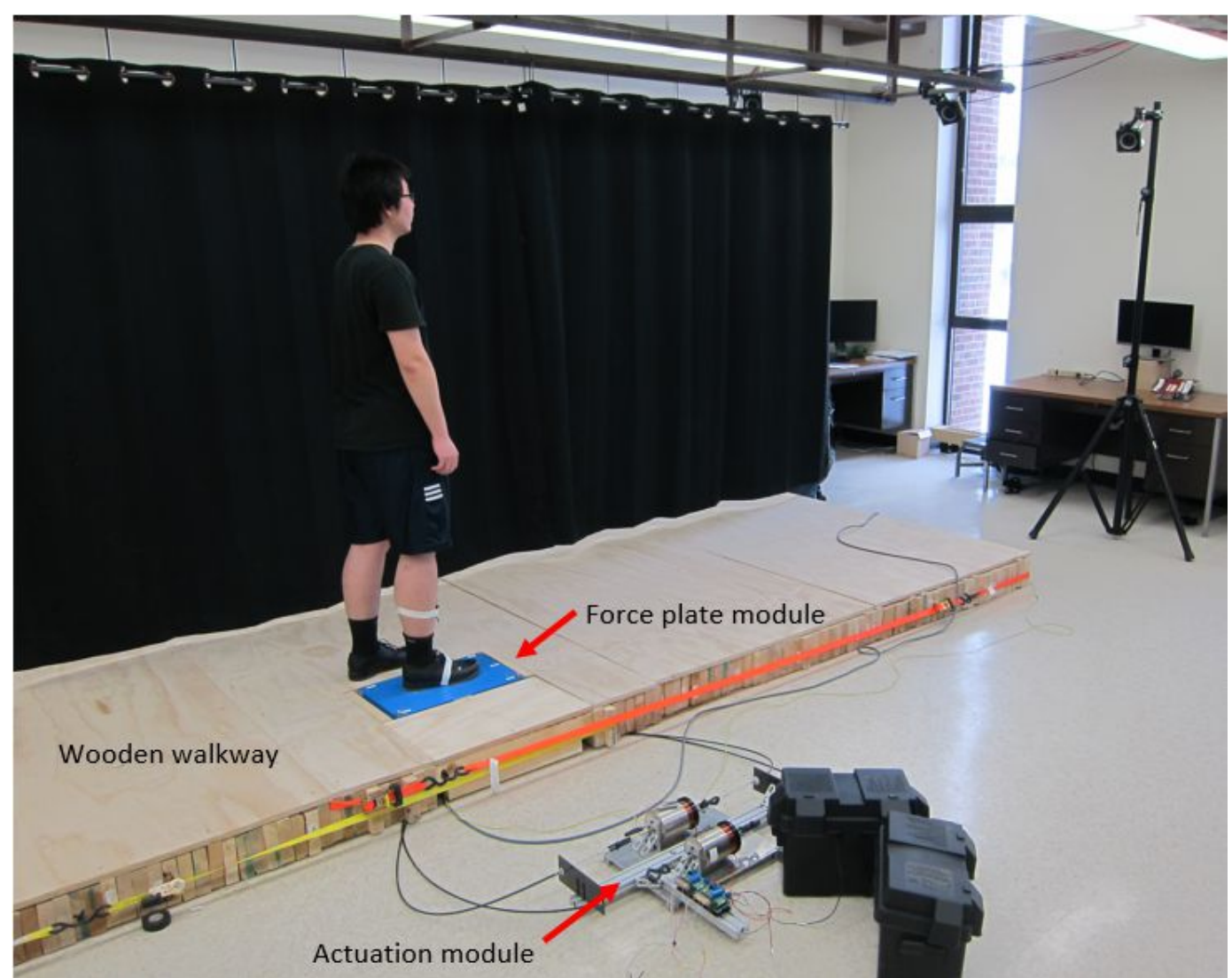

Figure 9.6. Instrumented walkway with the force plate module and actuation module installed.

\subsection{SYSTEM VALIDATION USING A MOCKUP}

\subsubsection{MOCKUP DESIGN}

To validate the instrumented walkway and vibrating platform capability for estimation of the mechanical impedance of a known system, an experiment with a mockup ankle-foot was conducted (Fig. 9.7). The mockup consisted of a heavy-duty spring rated at $136 \mathrm{kN} / \mathrm{m}$ in compression attached to a wooden foot and a support frame. The support frame is attached firmly to the walkway, preloading the spring $600 \mathrm{~N}$ to simulate the weight of a person and to assure the wooden foot is in contact with the force plate with no slippage. To record the angular displacement of the mockup ankle, infrared markers were installed on the force plate and the supporting 
frame (3 markers each) which can be regarded as the equivalent of the human foot and shin on human subjects, respectively.

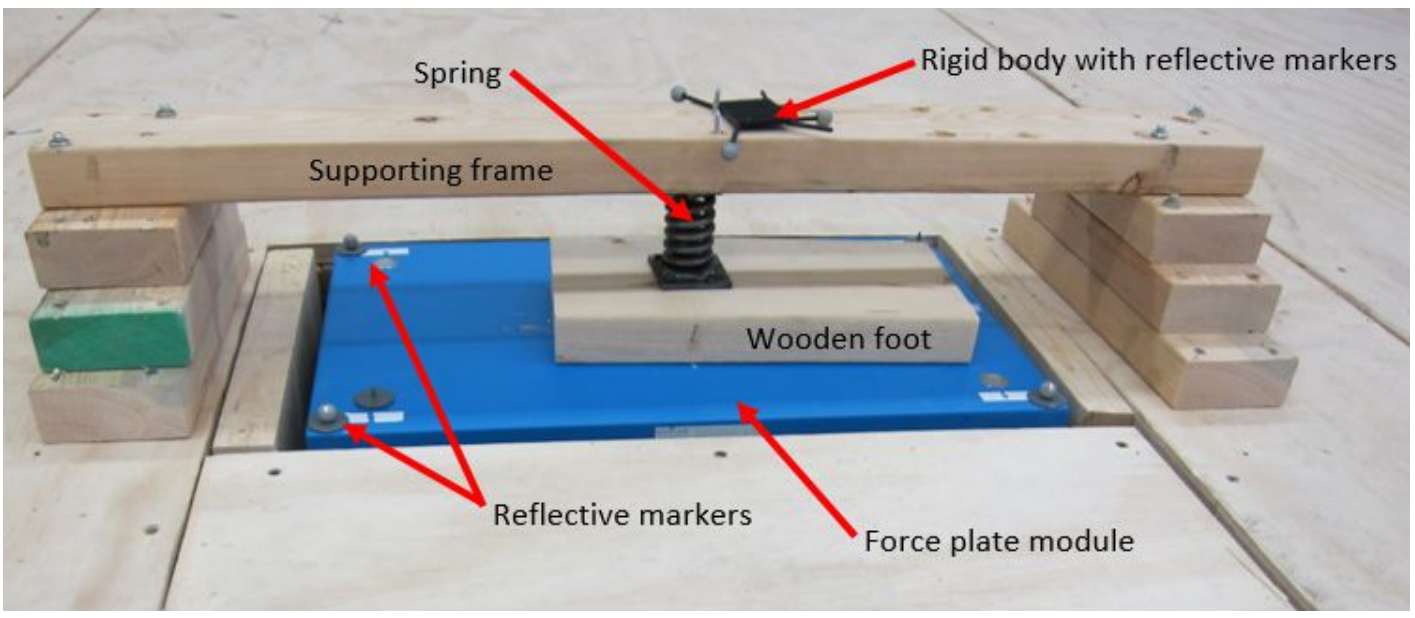

Figure 9.7. Mockup system is installed on top of force plate module for estimation of its impedance.

\subsubsection{ANKLE CENTER OF ROTATION CALCULATION}

The torque calculation, as shown in equations 3 and 4, requires the position of the center of rotation of the ankle (AC) in the force plate coordinate system. The ankle center of rotation is the instantaneous center of rotation of the foot with respect to the shin. The point $\mathrm{AC}$ can be transformed from the ankle center of rotation in the global coordinate system ( $\left.P^{\text {ankle }}\right)$ given the motion capture camera system measurements. To calculate $P^{a n k l e}$ it was assumed that the mockup foot and the shin segments are rigid bodies connected by a spherical joint. This kinematic constraint enforces the existence of a single pivot between the two rigid bodies. This pivot is the ankle center, as shown in Fig. 9.8. In humans the ankle center of rotation is not identical to a spherical joint. The human ankle is composed of the talocrural joint and the subtalar joint, and these joints are not aligned with the anatomical axes. The proper calculation of each instantaneous center of rotation is not trivial, and for simplicity, in this work the human ankle joint was approximated as a spherical joint. 


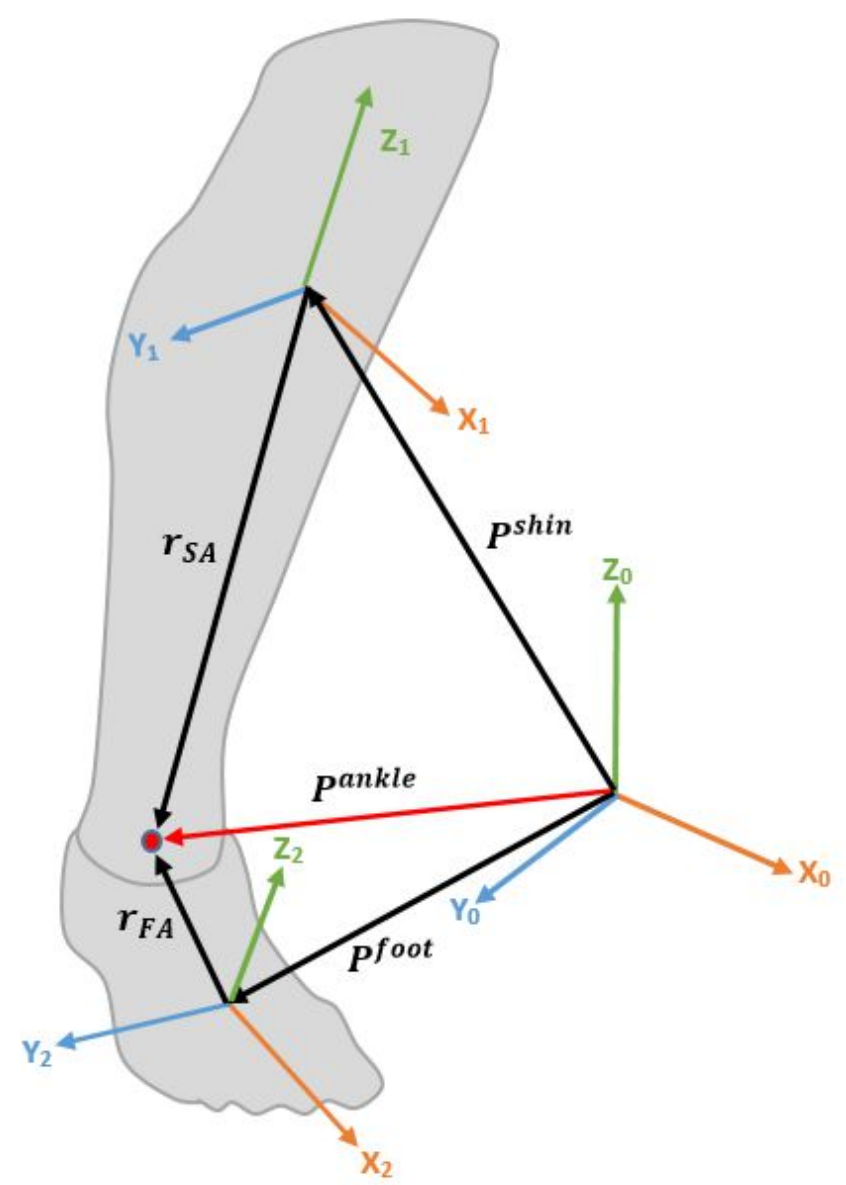

Figure 9.8. Shin and foot segments are represented as rigid bodies in their own coordinate systems. The common point between the foot and shin coordinate systems is at the intersection of the vectors $r_{S A}$ and $r_{F A}$ that are defined in the shin (X1Y1Z1) and ankle (X2Y2Z2) coordinate systems, respectively. The vectors $P^{\text {foot }}$ and $P^{\text {shin }}$ are vectors that define the position of the foot and shin coordinate systems in the global coordinate system (X0Y0Z0), respectively.

The vector $r_{S A}$ defines the ankle's center of rotation in the shin coordinate system $\left(\mathrm{X}_{1} \mathrm{Y}_{1} \mathrm{Z}_{1}\right)$. Similarly, the vector $r_{F A}$ defines the ankle's center of rotation in the foot coordinate system $\left(\mathrm{X}_{2} \mathrm{Y}_{2} \mathrm{Z}_{2}\right)$. The vectors $r_{S A}$ and $r_{F A}$ can be defined in global coordinate system using the rotation matrices $\mathbf{R}^{\text {shin }}$ and $\mathbf{R}^{\text {foot }}$ respectively. The rotation matrices $\mathbf{R}^{\text {shin }}$ and $\mathbf{R}^{\text {foot }}$ are found from the motion capture camera 
system. The global position of the ankle center of rotation $P^{\text {ankle }}$ at any recorded instance $(i)$ is defined in Equations 5 and 6.

$$
\begin{aligned}
& P_{i}^{\text {ankle }}=P_{i}^{\text {shin }}+\mathbf{R}_{i}^{\text {shin }} r_{S A} \\
& P_{i}^{\text {ankle }}=P_{i}^{\text {foot }}+\mathbf{R}_{i}^{\text {foot }} r_{F A}
\end{aligned}
$$

where $P^{\text {shin }}$ and $P^{\text {foot }}$ are vectors that define the foot and shin coordinate systems in the global coordinate system. Even though both Equations 5 and 6 define the coordinates of the ankle center of rotation, using the data from the camera system results in a small difference between the two vectors $(\varepsilon)$ at each instance as seen in equation 7:

$\varepsilon_{i}=P_{i}^{\text {shin }}+\mathbf{R}_{i}^{\text {shin }} r_{S A}-\left(P_{i}^{\text {foot }}+\mathbf{R}_{i}^{\text {foot }} r_{F A}\right)$

Assuming there are $N$ recorded instances, the Equation 7 can be rewritten into a Linear Least Square problem using the position and orientation of all the recorded samples during the experiment as shown in equation 8:

$$
\Phi \beta+\Delta P=\varepsilon
$$

where $\boldsymbol{\Phi}$ is a $3 N \times 6$ matrix that contains all the $\mathbf{R}^{\text {shin }}$ and $\mathbf{R}^{\text {foot }}$ matrices, $\Delta P$ is a $3 N \times 1$ vector that contains all the $P^{\text {shin }}-P^{\text {foot }}$ vectors, and $\beta$ is a $6 \times 1$ vector that contains the optimal solution vectors $r_{S A}$ and $r_{F A}$ that minimizes the $3 N \times 1$ residual error vector $\varepsilon$. The elements of each term in equation 8 are as follows:

$$
\underbrace{\left(\begin{array}{ccc}
\mathbf{R}_{1}^{\text {shin }} & \mid & -\mathbf{R}_{1}^{\text {foot }} \\
\vdots & \mid & \vdots \\
\mathbf{R}_{N}^{\text {shin }} & \mid & -\mathbf{R}_{N}^{\text {foot }}
\end{array}\right)}_{\boldsymbol{\Phi}} \underbrace{\left(\begin{array}{c}
r_{S A} \\
r_{F A}
\end{array}\right)}_{\beta}+\underbrace{\left(\begin{array}{c}
P_{1}^{\text {shin }}-P_{1}^{\text {foot }} \\
\vdots \\
P_{N}^{\text {shin }}-P_{N}^{f o o t}
\end{array}\right)}_{\Delta P}=\varepsilon
$$

Vectors $r_{S A}$ and $r_{F A}$ can be calculated by minimizing the residual vector $\overrightarrow{\boldsymbol{\varepsilon}}$ using the least square method in equation 9. By substituting $r_{S A}$ and $r_{F A}$ in equation 5 or 6 , the ankle center of rotation $P^{\text {ankle }}$ can be found in the global coordinate system. In this paper, the average results from both equations 5 and 6 was determined and used for increased accuracy. The point $P^{a n k l e}$ was later transformed to the force 
plate coordinate system to obtain the point $\mathrm{AC}$ to be used in the ankle torque calculations.

\subsubsection{QUASI-STATIC STIFFNESS MEASUREMENT}

Two experiments were performed with the mockup to estimate its stiffness. The first experiment was a static measurement with the Bowden cables disconnected and the frame springs removed. A weight of $12.18 \mathrm{~kg}$ was placed on top of the mockup with its center of mass at 3 different distances from the universal joint in the longitudinal axis of the force plate (the equivalent of a DP torque) and 3 different distances in the lateral axis (the equivalent of an IE torque). The rotations of the force plate were calculated from manual measurements of the force plate deflection when the weight was on top of the mockup using a micrometer. The MATLAB ${ }^{\circledR}$ tool fitlm, was used to create a linear regression model between the angles and torques, resulting in a stiffness of $1626 \mathrm{Nm} / \mathrm{rad}$ in DP and $1443 \mathrm{Nm} / \mathrm{rad}$ in IE. Note that this experiment does not consider the shear force between the force plate and mockup foot.

The second experiment was a quasi-static experiment with the Bowden cables connected and the frame springs attached. A sine wave torque input was generated using the motors with a frequency of $0.1 \mathrm{~Hz}$. The forces applied to the mockup ankle were measured using the force plate and the resulting angular displacement were measured using the motion capture camera system. Both systems recorded the data at 300 samples per second. The experiment lasted 40 seconds or 4 full sine wave cycles. The experiment was conducted twice, once with a DP sine wave input, followed by an IE sine wave input. Similar to the previous experiment, the MATLAB $^{\circledR}$ tool fitlm, was used to create a linear regression model between the input angles and output torques. The plots of the torque versus the angle of the mockup are shown in Figs. 9.9(a) and 9.9(b) for DP and IE, respectively. In these plots, fitting a straight line to the data and calculating its slope would result in an estimation of the stiffness of the mockup. The quasi-static stiffness of the mockup 
was determined to be $1971 \mathrm{Nm} / \mathrm{rad}$ in DP and $1749 \mathrm{Nm} / \mathrm{rad}$ in IE. The R-squared values, which represent how close the data were to the fitted regression line, were 0.995 in DP and 0.988 in IE, showing that the model fits well with the experimental data. Removing the effect of shear in the torque calculation resulted in a stiffness of $1657 \mathrm{Nm} / \mathrm{rad}$ in DP and $1484 \mathrm{Nm} / \mathrm{rad}$ in IE. These values are within $1.9 \%$ and $2.8 \%$ for DP and IE, respectively compared to the static tests using a weight and micrometer.

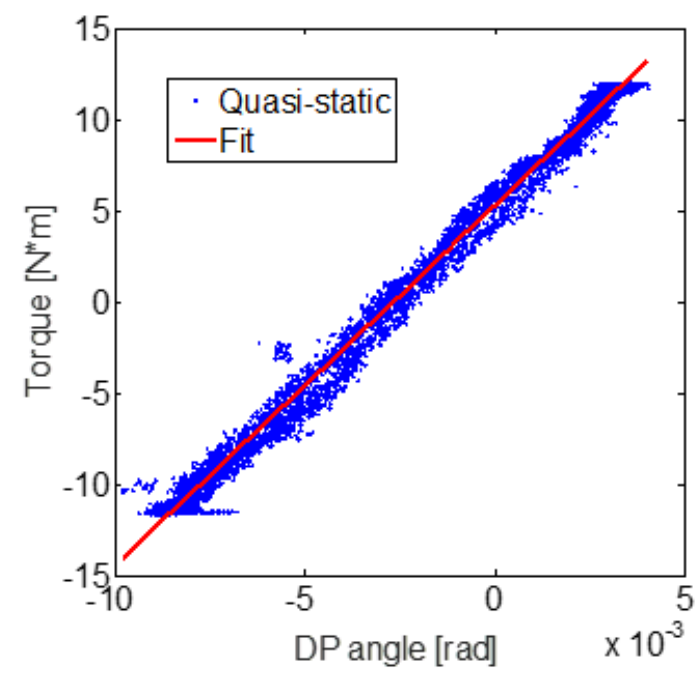

(a)

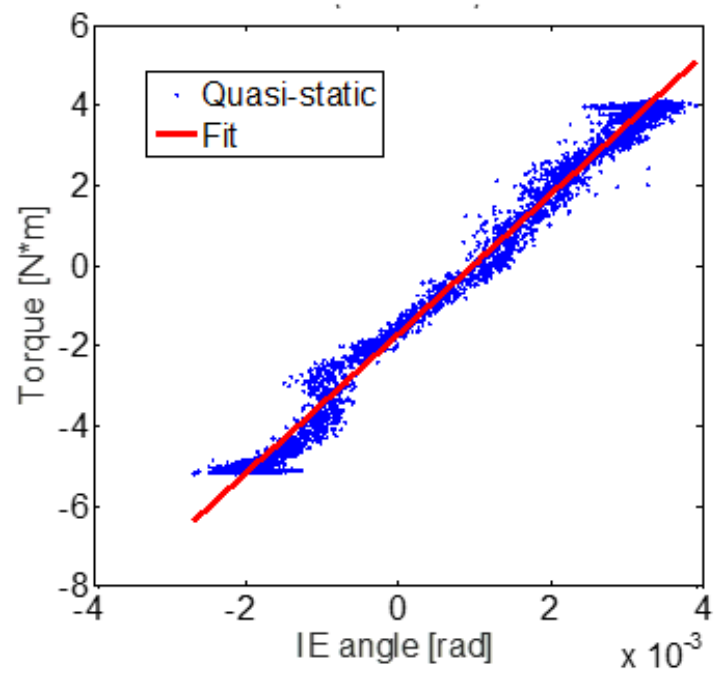

(b)

Figure 9.9. Plot of the torque versus angle of the quasi-static test of the mockup and straight line fit to the data. (a) Quasi-static stiffness in DP and linear fit with slope of $1971 \mathrm{Nm} / \mathrm{rad}$. (b) Quasi-static stiffness in IE and linear fit with slope of 1749 $\mathrm{Nm} / \mathrm{rad}$.

\subsubsection{MOCKUP IMPEDANCE MEASUREMENT USING A SINGLE-VARIABLE STOCHASTIC IDENTIFICATION METHOD}

The impedance can be defined as a transfer function $Z(f)$ relating input angles to the output torques at different frequencies $f$. A system's impedance is also can be defined by its inertia, damping and stiffness. The impedance measurement of the mockup consisted of generating uncorrelated pseudo-random torque perturbations at each motor, generating random torque inputs to the mockup in both DP and IE simultaneously. The bandwidth of the perturbations was set to $30 \mathrm{~Hz}$ and the 
sampling rate of the force plate and camera system was set to $300 \mathrm{~Hz}$. A torque input with motion output system is defined as a mechanical admittance. Assuming a linear dynamics, the admittance $Y(f)$ is a transfer function relating the input torque $\tau(f)$ and the output rotation of the mockup $\theta(f)$ (equation 10). The inverse of the admittance function is the impedance function $Z(f)$ relating the input angles to the output torques (equation 11).

$\theta(f)=Y(f) \tau(f)$

$Z(f)=Y^{-1}(f)$

Multiplying both sides of equation 10 by $Z(f)$ and substituting $Y^{-1}(f)$ to the right side and simplifying:

$Z(f) \theta(f)=\tau(f)$

And solving for the impedance $Z(f)$ :

$Z(f)=\frac{\tau(f)}{\theta(f)}$

where the values of $\tau(f)$ and $\theta(f)$ are determined from the experimental measurements. The force plate reading includes the effects from both the force plate and mockup and as a result the estimated impedance is a sum of the mockup and force plate impedances. To obtain the mockup impedance, it is necessary to subtract the impedance of the force plate from the estimated combined impedance. $Z(f)_{\text {mockup }}=Z(f)_{\text {mockup }+ \text { force plate }}-Z(f)_{\text {force plate }}$

The impedance of the plate $\left(Z(f)_{\text {force plate }}\right)$ was obtained from a similar experiment with no mockup on top of the force plate. All the force plate and camera system readings were due to the inertia of the force plate as there is no spring or damping acting on the force plate. The transfer function relating the input angles and output torques was calculated using a stochastic identification method. MATLAB ${ }^{\circledR}$ function tfestimate using a periodic Hamming window with a length of 512 samples and 50\% overlap. A fast Fourier transform (FFT) of 1024 samples was 
used, resulting in a frequency resolution of $0.293 \mathrm{~Hz}$. The coherence function was also calculated in MATLAB ${ }^{R}$ using the mscohere function to estimate the linear relationship between the input angles and output torques. The plot of the magnitude, phase, and coherence of the mockup in both DP and IE are shown in Fig. 9.10.

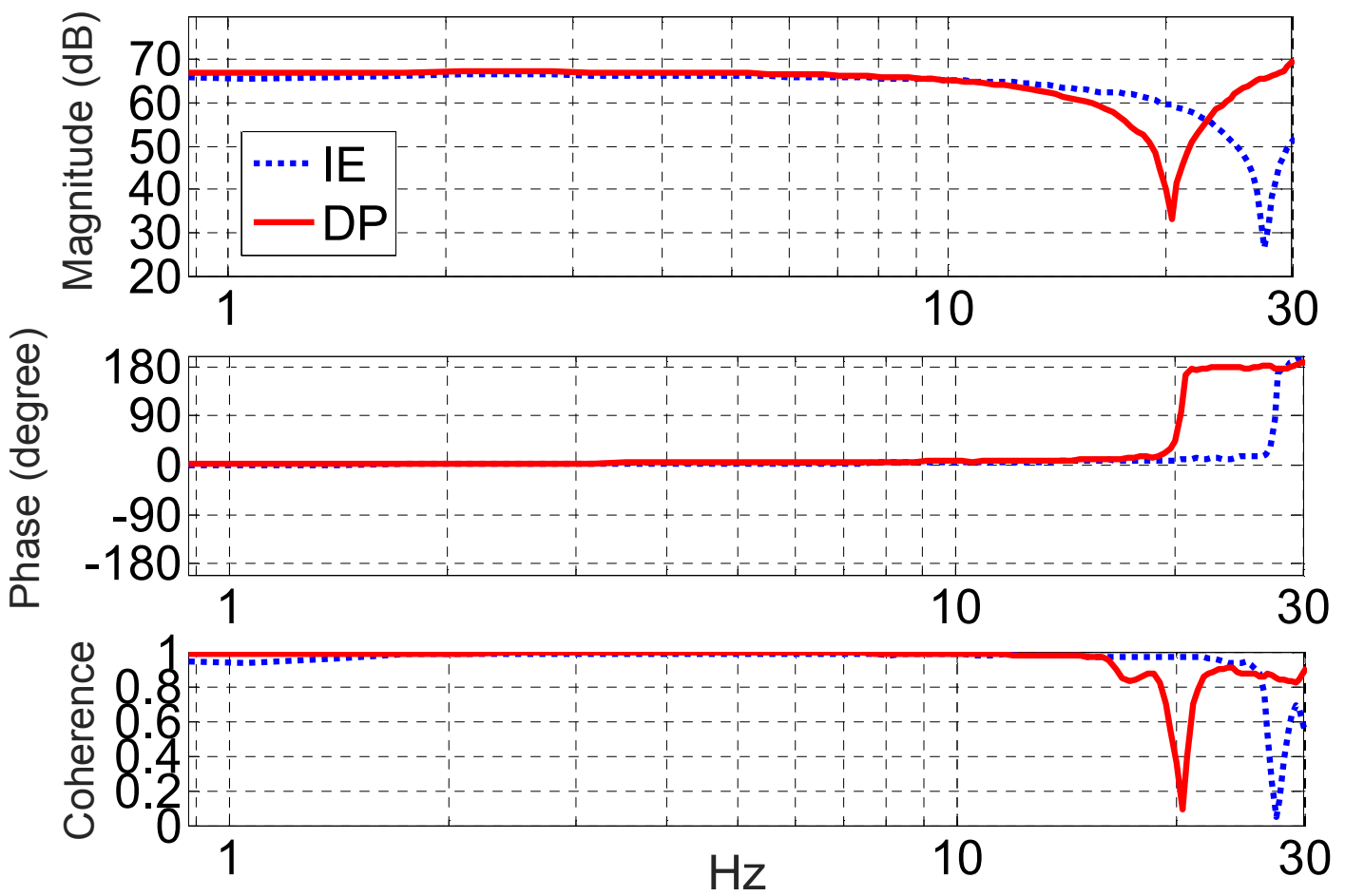

Figure 9.10. Plot of the mockup's impedance magnitude and phase, with the corresponding coherence using the single-variable stochastic identification method.

\subsubsection{MOCKUP IMPEDANCE MEASUREMENT USING A MULTI-VARIABLE STOCHASTIC IDENTIFICATION METHOD}

A similar approach can be used to calculate the impedance of the mockup using a multi-variable stochastic identification method. This approach takes into the consideration that the input motion in DP may affect the output torque in IE and vice versa due to the coupled dynamics (108). The plot of the impedance of the mockup using a multi-variable approach is shown in Fig. 9.11. Minor differences were observed when the two methods were compared since the mockup has no 
mechanical coupling between DP and IE. The average relative magnitude error between the single-variable and multi-variable methods averaged across all frequencies in the $0.7 \mathrm{~Hz}$ to $30 \mathrm{~Hz}$ was $1.73 \%$ in DP and $0.33 \%$ in IE, respectively. In the human ankle however, the amount of coupling between DP and IE during standing and walking is unknown, suggesting a multi-variable impedance estimation approach to be more plausible.

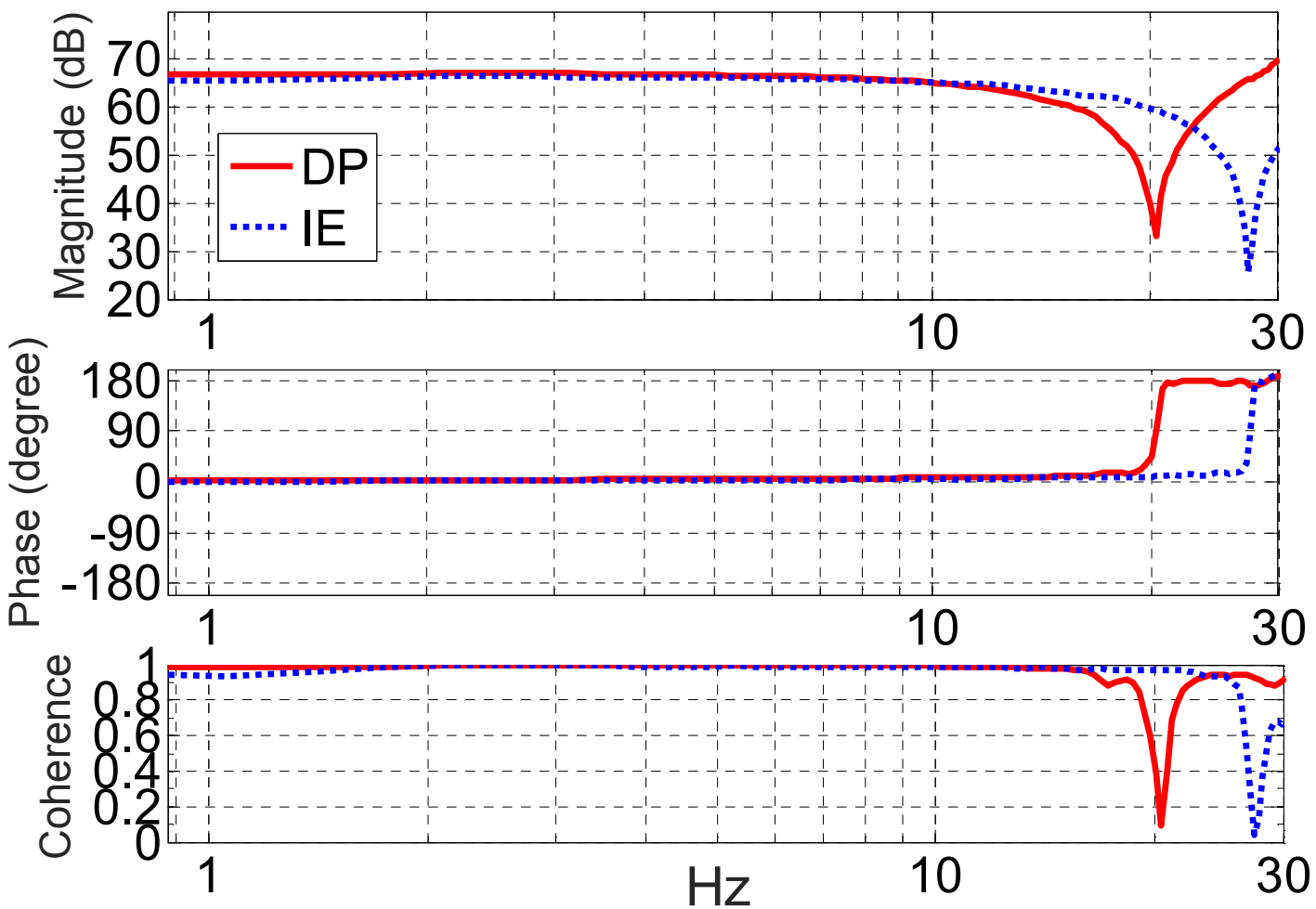

Figure 9.11. Plot of the mockup's impedance magnitude and phase, with the corresponding coherence using the multi-variable stochastic identification method.

\subsection{PRELIMINARY EVALUATION OF THE TIME-VARYING MECHANICAL IMPEDANCE OF THE HUMAN ANKLE}

A preliminary experiment using a sample human subject was designed and conducted to identify the time-varying mechanical impedance of the human ankle during straight walking at normal speeds. Ensemble-based linear time-varying system identification method was used (106). This method has previously been used 
by Lee et al. for the characterization of human ankle mechanical impedance in two DOF during pre-swing, swing, and early stance of the gait (58) using a wearable rehabilitation robot (Anklebot).

In this method, multiple realizations $\mathrm{R}$ of the data are required (in this case $\mathrm{R}$ is the number of steps) with the same fundamental time-varying behavior. The average of all realizations can be subtracted from each realization to remove the fundamental time-varying behavior due to normal walking. This removes the dynamics of normal walk, which are similar and repeating at each step. This method provides the torques and angles due to the input perturbations to the ankle that are random at each step, and can be used for estimation of the mechanical impedance of the ankle.

Since each step may have different lengths, the data is normalized to equal lengths with minimal effect on the results (58). Each normalized step produces vectors of $\mathrm{N}$ samples in length of input angular displacements $\Theta_{\mathrm{r}}$ and output torques $\tau_{\mathrm{r}}$. At each sample $\mathrm{i}$, (for $\mathrm{i}$ from 1 to $\mathrm{N}$ ), the method consists of solving a matrix equation (equation 15) involving estimates of the input autocorrelation $\Phi_{\theta \theta}(i)$ (equation 16) and the input-output cross-correlation $\Phi_{\tau \theta}(\mathrm{i})$ (equation 17) across all the steps R (106). Solving this matrix results in an impulse response function (IRF) $\mathrm{h}(\mathrm{i})$ with a finite leg length of $\mathrm{L}$ samples representing the dynamics of the system at the sample $\mathrm{i}$. In equations 16 and 17, $\mathrm{j}$ and $\mathrm{k}$ are the leg length (L) indices (for $\mathrm{j}$ and $k$ from 0 to $L-1)$. Note that $h(i, j)=0$ for $j \geq L$.

$$
\begin{aligned}
& \mathrm{h}(\mathrm{i})=\frac{\Phi_{\theta \theta}(\mathrm{i})^{-1} \Phi_{\tau \theta}(\mathrm{i})}{\Delta \mathrm{t}} \\
& \Phi_{\theta \theta}(\mathrm{i})=\frac{1}{\mathrm{R}} \sum_{\mathrm{r}=1}^{\mathrm{R}} \theta_{\mathrm{r}}(\mathrm{i}-\mathrm{j}) \theta_{\mathrm{r}}(\mathrm{i}-\mathrm{k}) \\
& \Phi_{\tau \theta}(\mathrm{i})=\frac{1}{\mathrm{R}} \sum_{\mathrm{r}=1}^{\mathrm{R}} \tau_{\mathrm{r}}(\mathrm{i}) \theta_{\mathrm{r}}(\mathrm{i}-\mathrm{k})
\end{aligned}
$$


In this experiment, 400 steps were conducted $(\mathrm{R}=400)$. The ankle angles and torques were sampled at $300 \mathrm{~Hz}$. Only the data recorded during the stance phase was used, as there is no torque recorded during the swing phase when the foot is not in contact with the force plate. A metronome was used to help the subject keep a constant speed of gait. The metronome was set at the user's preferred speed of walk, but some amount of variation was inevitable. To remediate the variations in gait, outliers in stride length and in the ankle angle (DP angles for DP data processing and IE angles for IE data processing) were removed. Specifically, the 5\% longest and shortest steps were rejected before normalization in length, and the 5\% steps that showed the largest amount of maximum rotation and the $5 \%$ with the least amount of maximum rotation were removed resulting in a total of 324 steps. The steps were normalized in length to the size of the average length (311 samples). To remove the dynamics of the ankle due to the normal walk, the average angles across all realizations and the average torques across all realizations were removed from each realization angles and torques, respectively. This removed the fundamental non-perturbed dynamics of the ankle due to normal walk, resulting in the random input-output data due to the perturbations applied to the ankle. The resultant torques and angles were run through equations 15-17 to calculate the IRF. The leg length L was chosen at 63 samples ( 0.21 seconds). It was chosen to be long enough for the IRF to converge to zero. This method has a limitation at heel strike. Because a leg length of 63 samples was used, the leg indices $\mathrm{j}$ and $\mathrm{k}$ can be as long as 62 samples. At heel strike, there is no prior information of the ankle torques, as the foot does not touch the force plate before heel strike and the ankle torque cannot be calculated; as a result, the method cannot be used until L samples after the heel strike. Thus the IRF were calculated from sample $i=63$ to the final sample $i=311$, which corresponds to $20 \%$ to $100 \%$ of the stance phase. The impulses were grouped and averaged in $10 \%$ interval section of the stance phase (31 impulses in each interval) as shown in Fig. 9.12 for DP and Fig. 9.13 for IE. 


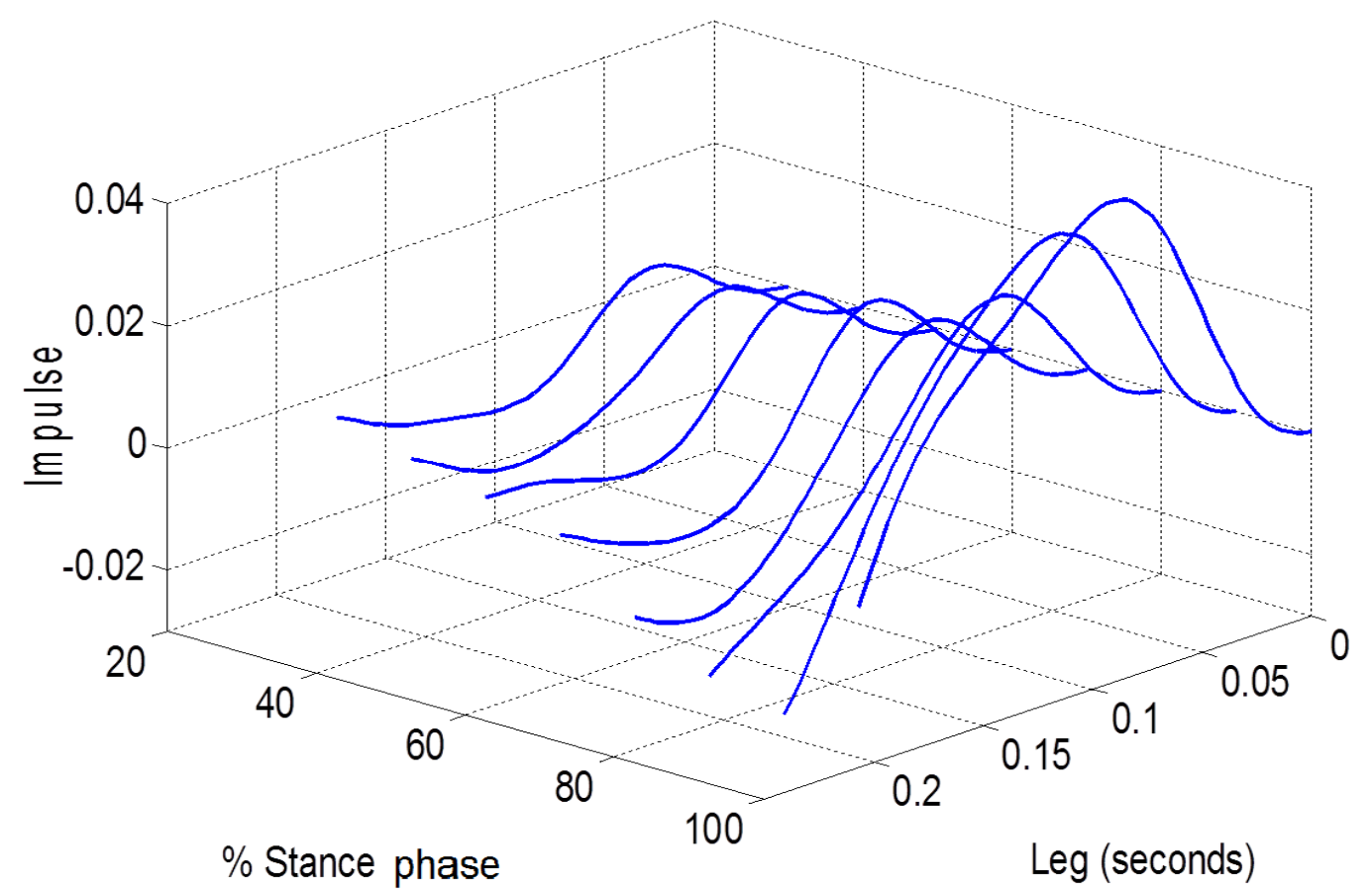

Figure 9.12. IRF of the time-varying mechanical impedance of a sample human ankle in DP during a straight walk.

The stance was divided into 10 segments of equal length (each containing 10\% of the stance phase) and the impulse response functions in each segment were averaged. To estimate the equivalent spring and damping, a second order model was fit to each averaged impulse response functions. The MATLAB $^{\circledR}$ command filt was used to specify discrete transfer functions. Assuming a second order system to describe the ankle, only the first 2 most relevant states were needed, so all the states with relevance greater than 3 were removed. The resulting model was a second order discrete model that was converted to a continuous model using MATLAB ${ }^{\circledR}$, $d 2 c$ command with the Tustin approximation. The results obtained still needs verification; which can possibly be done a using mockup with time varying stiffness. The resulting damping and stiffness can be observed in Tables 9.1 and 9.2 for DP and IE, respectively. 


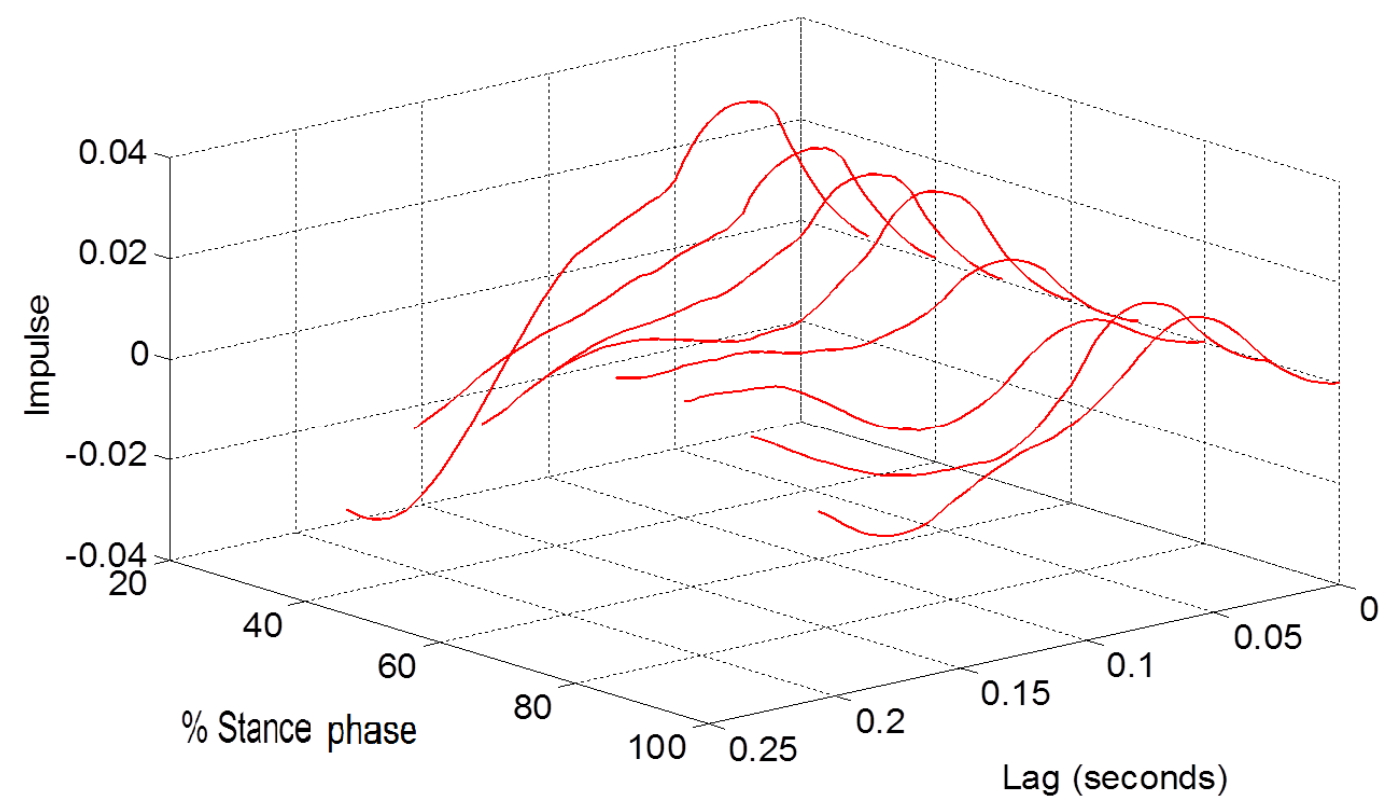

Figure 9.13. IRF of the time-varying mechanical impedance of a sample the human ankle in IE during a straight walk.

Table 9.1: Damping and stiffness calculated from the IRF of the time-varying mechanical impedance of the human ankle in DP during straight walk.

\begin{tabular}{|c|c|c|}
\hline DP \% Stance & Damping Nm/(rad/s) & Stiffness (Nm/rad) \\
\hline $20-30 \%$ & 30 & 2634 \\
\hline $30-40 \%$ & 28.7 & 2345 \\
\hline $40-50 \%$ & 26.4 & 2032 \\
\hline $50-60 \%$ & 38.9 & 2798 \\
\hline $60-70 \%$ & 22.5 & 1940 \\
\hline $70-80 \%$ & 12.4 & 819 \\
\hline $80-90 \%$ & 10.1 & 415 \\
\hline $90-100 \%$ & 11.8 & 213 \\
\hline
\end{tabular}


Table 9.2: Damping and stiffness calculated from the IRF of the time-varying mechanical impedance of the human ankle in IE during straight walk.

\begin{tabular}{|c|c|c|}
\hline IE \% Stance & Damping Nm/(rad/s) & Stiffness $(\mathrm{Nm} / \mathrm{rad})$ \\
\hline $20-30 \%$ & 9.5 & 655 \\
\hline $30-40 \%$ & 23.6 & 533 \\
\hline $40-50 \%$ & 27.3 & 476 \\
\hline $50-60 \%$ & 28.3 & 417 \\
\hline $60-70 \%$ & 42.5 & 481 \\
\hline $70-80 \%$ & 23.0 & 3940 \\
\hline $80-90 \%$ & 21.7 & 1911 \\
\hline $90-100 \%$ & 22.7 & 1212 \\
\hline
\end{tabular}

\subsection{DISCUSSION}

The ankle-foot mockup stiffness was estimated using a weight and a micrometer and the results were compared to its estimated quasi-static stiffness using the torque generated by its motors and the camera system. The stiffness of the mockup was within $1.9 \%$ for DP and $2.8 \%$ for IE when comparing these two methods. This shows that the camera system and force plate provided reliable information to calculate the ankle torques, angles, and center of rotation that used in the impedance estimation. Also, it showed that the torque generated by the frame springs and the torque generated by the motors are not present in the stiffness measurement as this elements were not used in the weight and micrometer tests but both experiments resulted in similar stiffness. This shows a proper decoupling of theses torques from the force plate readings,

In the impedance measurement at $2 \mathrm{~Hz}$, the magnitude of the impedance was estimated at $65.97 \mathrm{~dB}(1988 \mathrm{Nm} / \mathrm{rad})$ in $\mathrm{DP}$ and $64.81 \mathrm{~dB}(1740 \mathrm{Nm} / \mathrm{rad})$ in IE using the single-variable stochastic identification method. These values are within $0.9 \%$ and $0.5 \%$ of the values estimated using the quasi-static impedance estimation, respectively. Using the multi-variable stochastic identification method, the 
impedance magnitude estimations were $65.90 \mathrm{~dB}(1972 \mathrm{Nm} / \mathrm{rad})$ for DP and 64.76 $\mathrm{dB}(1730 \mathrm{Nm} / \mathrm{rad})$ which are within $0.05 \%$ and $1.1 \%$ of the values estimated using the quasi-static impedance estimation, respectively. Ideally, both stiffness values in DP and IE should be the same; however, the spring was welded to steel plates to allow it to be bolted down to the wood frame and foot block that changed its properties resulting in different stiffness of the mockup in DP and IE.

The coherence values for the estimated impedance of the mockup from both single-variable and multi-variable stochastic method were above 0.9 up to $15 \mathrm{~Hz}$, showing a linear relationship between angles and torques. The mockup had natural frequencies at $20.4 \mathrm{~Hz}$ in DP and $25.7 \mathrm{~Hz}$ in IE, which are visible by the sharp drop in the magnitude plots due to the low damping ratio of the mockup, a $180^{\circ}$ phase increase, and the significant drops in coherence at those frequencies. The lower value of natural frequency of the mockup in DP when compared to IE was expected as the mockup foot has larger mass moment of inertia in DP than it has in IE and the natural frequency of a system has an inverse relationship with the mass moment of inertia. This shows the capability of the system to detect a system inertia, damping, and stiffness up to $30 \mathrm{~Hz}$.

Since the mockup consists of a steel spring, no coupling was expected between DP and IE. As a result, the averaged magnitude error between the single-variable and multi-variable methods was small with values of $1.73 \%$ in DP and $0.33 \%$ in IE with the maximum error found at $18.3 \mathrm{~Hz}$ and $26.4 \mathrm{~Hz}$ for DP and IE, respectively. The maximum relative error was found near the natural frequencies, which is expected since the coherence drops significantly at the natural frequencies.

Preliminary results of the time-varying mechanical impedance of the human ankle using the ensemble-based linear time-varying system identification method shows the capability of the system and the method through most of the stance phase. In DP, the highest stiffness and damping were observed at 50-60\% of the stance phase with damping value of $38.9 \mathrm{Nm} /(\mathrm{rad} / \mathrm{s})$ and stiffness of $2798 \mathrm{Nm} / \mathrm{rad}$. The last 
$10 \%$ of the stance showed the least amount of stiffness and damping with values of $11.8 \mathrm{Nm} /(\mathrm{rad} / \mathrm{s})$ for damping and $213 \mathrm{Nm} / \mathrm{rad}$ for stiffness. Although the ankle generates the most power at push-off, it showed the least amount of stiffness at this portion of the stance phase. The impedance of a joint is a function of muscle cocontraction of antagonistic muscles, and the amount of power generated by the ankle is not an independent indicator of the joint stiffness. In IE, the largest stiffness was observed at $70-80 \%$ of stance with value of $3940 \mathrm{Nm} / \mathrm{rad}$ and the largest damping was at $60-70 \%$ of the stance with value of $42.59 \mathrm{Nm} /(\mathrm{rad} / \mathrm{s})$. The minimum values were found at $20-30 \%$ of the stance phase with values of $9.5 \mathrm{Nm} /(\mathrm{rad} / \mathrm{s})$ and $655 \mathrm{Nm} / \mathrm{rad}$ for damping and stiffness respectively. Different methods, other than the ensemble-based linear time-varying system identification method, may be used to identify the impedance of the ankle at heel strike and to validate the results from the presented method. One approach is to apply few single ramp perturbations to the ankle as described in (49) at each step instead of continuous random perturbations. This is an ongoing research.

\subsection{CONCLUSION}

This paper described the construction and preliminary evaluation of a 2-DOF instrumented vibrating platform that is aimed for impedance estimation of the human ankle during standing and walking in arbitrary directions in both the sagittal and frontal planes. The vibrating platform was capable of generating torques similar to the human ankle in both DOF. A force plate and motion capture camera system were used for recording the data necessary for the calculation of the ankle's torques and angles required for the ankle's impedance estimation in both dorsiflexionplantarflexion and inversion-eversion directions. The construction of the device was explained in details and the mathematical approach required for the calculation of the ankle torques and angles were presented. Additionally, the analytical and numerical approaches for impedance estimation was presented. A system validation experiment with a mockup was developed to evaluate the system capability to 
estimate the impedance of a physical system in two DOF. The impedance of the mockup at $2 \mathrm{~Hz}$, using a multi-variable stochastic identification method, was within $0.05 \%$ and $1.1 \%$ of its quasi-static stiffness in dorsiflexion-plantarflexion and inversion-eversion directions, respectively. The experiment showed that the developed system was capable of properly estimating the impedance of the system by identifying its inertia, damping, and stiffness of the mockup. Preliminary results of the time-varying mechanical impedance of the human ankle using ensemblebased linear time-varying system identification methods show the capability of the system and the method through most of the stance phase, except at early stance phase. 


\section{0-MECHANICAL IMPEDANCE OF THE NON- LOADED LOWER LEG WITH RELAXED MUSCLES IN THE TRANSVERSE PLANE}

\subsection{ABSTRACT}

This paper describes the protocols and results of the experiments for the estimation of the mechanical impedance of the humans' lower leg in the ExternalInternal (EI) direction in the transverse plane under non-load bearing condition and with relaxed muscles. The objectives of the estimation of the lower leg's mechanical impedance are to facilitate the design of passive and active prostheses with mechanical characteristics similar to the humans' lower leg, and to define a reference that can be compared to the values from the patients suffering from spasticity. The experiments were performed with 10 unimpaired male subjects using a lower extremity rehabilitation robot (Anklebot, Interactive Motion Technologies, Inc.) capable of applying torque perturbations to the foot. The subjects were in a seated position, and the Anklebot recorded the applied torques and the resulting angular movement of the lower leg. In this configuration the recorded dynamics are due mainly to the rotations of the ankle's talocrural and the subtalar joints, and any contribution of the tibiofibular joints and keen joint. The dynamic mechanical impedance of the lower leg was estimated in the frequency domain with an average coherence of 0.92 within the frequency range of 0 to $30 \mathrm{~Hz}$, showing a linear correlation between the displacement and the torques within this frequency range under the conditions of the experiment. The mean magnitude of the quasi-static stiffness of the lower leg (the impedance magnitude averaged in the range of 0-1 $\mathrm{Hz}$ ) was determined as $4.9 \pm 0.74 \mathrm{Nm} / \mathrm{rad}$. The direct estimation of the quasi-static stiffness of the lower leg results in the mean value of $5.8 \pm 0.81 \mathrm{Nm} / \mathrm{rad}$. An analysis of variance (ANOVA) shows that the estimated values for the quasi-static stiffness from the two experiments are not statistically different.

*The material contained in this chapter is in preparation for submission to a journal. 


\subsection{INTRODUCTION}

The ankle is the first major joint that transfers the ground reaction forces to the rest of the body during walking. Reaction forces allow for propulsion and the changing of direction of gait. Activities of Daily Leaving (ADLs) involve different tasks requiring the lower leg to function in all the anatomical planes. For example, during normal walking, walking on inclined planes, turning around corners, avoiding obstacles, and climbing/descending stairs, the lower leg dynamics change continuously to accommodate for these different maneuvers and conditions of agile gait. Studies of four representative daily activities show that, depending on the activity, turning steps may account for an average of $25 \%$ (range $8 \%$ to $50 \%$ ) of all daily steps (1). Therefore, the development of ankle-foot prostheses should take the mechanical characteristics of the human lower leg into consideration.

Currently, available powered ankle-foot prostheses focus on improving mobility by powering the ankle joint in the sagittal plane; however, there is substantial ankle function in all anatomical planes, even during straight walk on level ground $(5,35$, 109). Ankle-foot prostheses with anthropomorphic characteristics may improve the metabolic cost while generating a more comfortable gait and decreasing the secondary injuries due to overuse or misuse of other joints. These may increase mobility and activity levels, reduce the likelihood of obesity and cardiovascular diseases, and overall improve the quality of life in amputees.

The ankle and lower leg kinetics and kinematics show significant variability when comparing the angles and torques during straight walking and sidesteps cutting (a step where the leading leg pushes the body sideways near or at $45^{\circ}$ to avoid an obstacle on the ground while walking forward) in both EI and InversionEversion (IE). It has been shown that during a sidestep cutting maneuver at normal walking speed, the ankle torque in the lateral direction at the push off phase increased more than 6 times compared to walking on a straight path (5). This result indicated a torque in the transverse plane is transferred from the human body to the 
ground through the ankle during walking on a straight path. The amount of transferred torque is larger during turning. On the other hand, the ankle angles during these two walking scenarios show no statistical differences, which indicates that a higher stiffness of the ankle is required for turning maneuvers. This higher stiffness is necessary to transfer the torque from the hip joint to the ground, so the ground reaction torques would steer the body into the new walking direction. The ankle is capable of considerable movement in the transverse plane, allowing the body to rotate in the transverse plane while the foot remains in contact with the floor (110). This evidence suggests that the lower leg dynamics and its variable stiffness may play a significant role during walking and turning when the lower leg's muscles undergo co-contraction.

The ankle is composed of the talocrural and the subtalar joints, which are not aligned with the anatomical axes. The rotation of the ankle about each of these joints results in combined rotations in the anatomical reference frame. The talocrural joint combines dorsiflexion with lateral rotation and also combines plantarflexion with medial rotation. The subtalar joint combines dorsiflexion, eversion, and external rotation and additionally it combines plantarflexion, inversion, and internal rotation (13). The tibia and the talus of the foot form the talocrural joint, and the tibia is in contact with the fibula at their lower and upper ends as the tibiofibular joints. The movements of the tibiofibular joints are small and usually neglected in gait analysis (111). In the presented experiment; however, the kinematics and kinetics, hence the impedance of the lower leg in the transverse plane is presented as a combination of the talocrural and subtalar joints' functions and any contribution from the tibiofibular and knee joints. During the experiments presented in this paper, the angular movement of the lower leg in the global reference frame and the applied torque by the Anklebot were recorded. Therefore, the estimated impedance is the lower leg rotational impedance in the transverse plane contributed by the ankle and lower leg musculoskeletal system. The stiffness of the leg in the transverse plane is also affected by the angle of the knee joint; when the leg is 
stretched, the hip rotation in the transverse plane is present at the lower leg and changes the impedance measurement. Since the tests were performed with the knee at a $90^{\circ}$ angle, the effects of the hip joint on the results were not present. Therefore, the results are merely valid for the conditions of the performed experiments.

The mechanical impedance of a system is defined as the evoked torque due to input motion perturbations, and it is a function of the systems mass, damping, and stiffness. The ankle impedance in the frontal and sagittal planes have been previously studied with relaxed and co-contracting muscles under no-load condition using single and multivariable stochastic identification approaches $(50,51,54,79$, $80,82,83,85,106,112-115)$. The ankle impedance variation in the sagittal plane during the foot-flat sub-phase of stance was estimated using a perturbation platform(104). Additionally, the time-varying dynamic mechanical impedance of the ankle in sagittal and frontal planes during pre-swing, swing, and early stance of the gait was studied using the Anklebot (116). Both those studies showed consistent time-varying behavior of the ankle mechanical impedance during gait.

Powered lower extremity prostheses usually have one degree of freedom (DOF) in the sagittal plane $(27,69,70)$. The authors have developed a 2-DOF powered ankle-foot prosthesis with two controllable DOFs in the sagittal and frontal planes $(117,118)$. The design of powered ankle-foot prostheses generally considers the lower leg and ankle to be fixed in the transverse plane, since the hip joint is capable of generating the majority of torques that is transferred to the ground for steering and turning. Nevertheless, the ankle and lower leg rotate in the transverse plane during the gait and should be designed as a compliant joint in this anatomical plane. Recently, Olson et al. developed a transtibial prosthesis with active control in the transverse plane (11). This device is capable of generating torque in single DOF in the transverse plane and uses an impedance controller. A better understanding of the impedance of the lower leg in the transverse plane may lead to the development of ankle-foot prostheses that can properly mimic the mechanical characteristics of the human lower leg in EI (119). Compliance in the transverse plane may reduce the 
painful shear stresses on the residual limbs (120). Variable stiffness mechanisms could be developed to mimic the time-varying impedance of the ankle and lower leg, facilitating ADLs, reducing secondary injuries, and improving overall quality of life for lower extremity amputees.

This paper presents the methods and the results of the estimation of the dynamic mechanical impedance and quasi-static stiffness of the human lower leg under noload bearing condition and with relaxed muscles in the transverse plane. This study is aimed to improve our understanding of the dynamics of the lower leg function by developing experiments that may lead to better explaining how its muscle activation may modulate its mechanical impedance in the transverse plane. The practical benefits of this information may lead to characterizing the primary design parameters for the development of the ankle-foot prostheses with anthromporphic characteristics. Additionally, the results can be used as a reference for comparing the results from patients suffering from spasticity due to stroke or patients with multiple sclerosis. Lee et al. (121) compared the results of the quasi-static impedance of the ankle in individual suffering from stroke and multiple sclerosis with unimpaired subjects. They showed some quantitative differences in the results of the mechanical impedance of the ankle in dorsiflexion-plantarflexion and inversion-eversion. An impedance estimation experiment may be used to examine the patients' level of impairment, or to monitor their progress during treatment.

In this paper, the experiments were performed using a lower extremity rehabilitation robot on 10 unimpaired male subjects. In the quasi-static test, the robot applied ramp perturbations at $0.4 \mathrm{rad} / \mathrm{sec}$ to the lower leg and the resultant lower leg torque was recorded. In the dynamic impedance estimation experiment, the robot applied random torque perturbations to the lower leg and a stochastic identification method was used. The stochastic identification method was used since it does not require any a priori information about the dynamics of the system, making it suitable for analysis of complex mechanical systems such as the human lower leg. The stochastic method presented has the advantage of applying equal 
amounts of energy at all frequencies within the studied frequency range. The paper first describes the experiment setup to use the Anklebot to apply perturbations to the lower leg. Next, the experiment protocol and results for dynamic impedance estimation and quasi-static stiffness estimation are presented and discussed.

\subsection{EXPERIMENT METHODOLOGY}

\subsubsection{Human SUBJECTS}

Ten male subjects with no self-reported neuromuscular and biomechanical disorders were recruited for the experiments (ages ranging from 23 to 28 years and body mass index (BMI) ranging from 22.4 to 30.0). The subjects gave written consent to participate in the experiment, which was approved by the Michigan Tech Institutional Review Board.

\subsubsection{EXPERIMENTAL SETUP}

A wearable lower extremity rehabilitation robot capable of applying controlled torque perturbations to the lower leg, Anklebot, was used to apply torque perturbations in EI direction. The Anklebot records the applied torques and the angular displacement of the lower leg as the result of the applied perturbations as described in detail by Roy et al. (88). The Anklebot is backdrivable with low friction; therefore, it allows the users to move their foot relative to the shank. It consists of two nearly parallel linear actuators attached to the leg (through a shoe) as seen on figure 1. Position information is provided by two Renishaw linear incremental encoders with a resolution of $5 \times 10^{-6} \mathrm{~m}$ mounted on the traction drives. Torque is measured by current sensors (Burr-Brown 1NA117P), which provide a measure of motor torque with a nominal resolution of $2.59 \times 10^{-6} \mathrm{Nm}$.

The Anklebot has been previously used for estimation of the ankle mechanical impedance in both dorsiflexion-plantarflexion (DP) and IE $(50-55,112,113,122)$. In these studies, the actuators were placed parallel to the shin and aligned approximately between the knee and the ball of the foot. The Anklebot was attached 
to the leg through a knee brace and a modified shoe. The weight of the Anklebot was supported by mounting the knee brace to the chair or by hanging on a horizontal bar. In this configuration, the sum of the actuator forces generated a DP torque, and their difference generated an IE torque. The maximum capacity of the Anklebot in applying controllable torques in DP is $23 \mathrm{Nm}$ and in IE is $15 \mathrm{Nm}$, simultaneously.

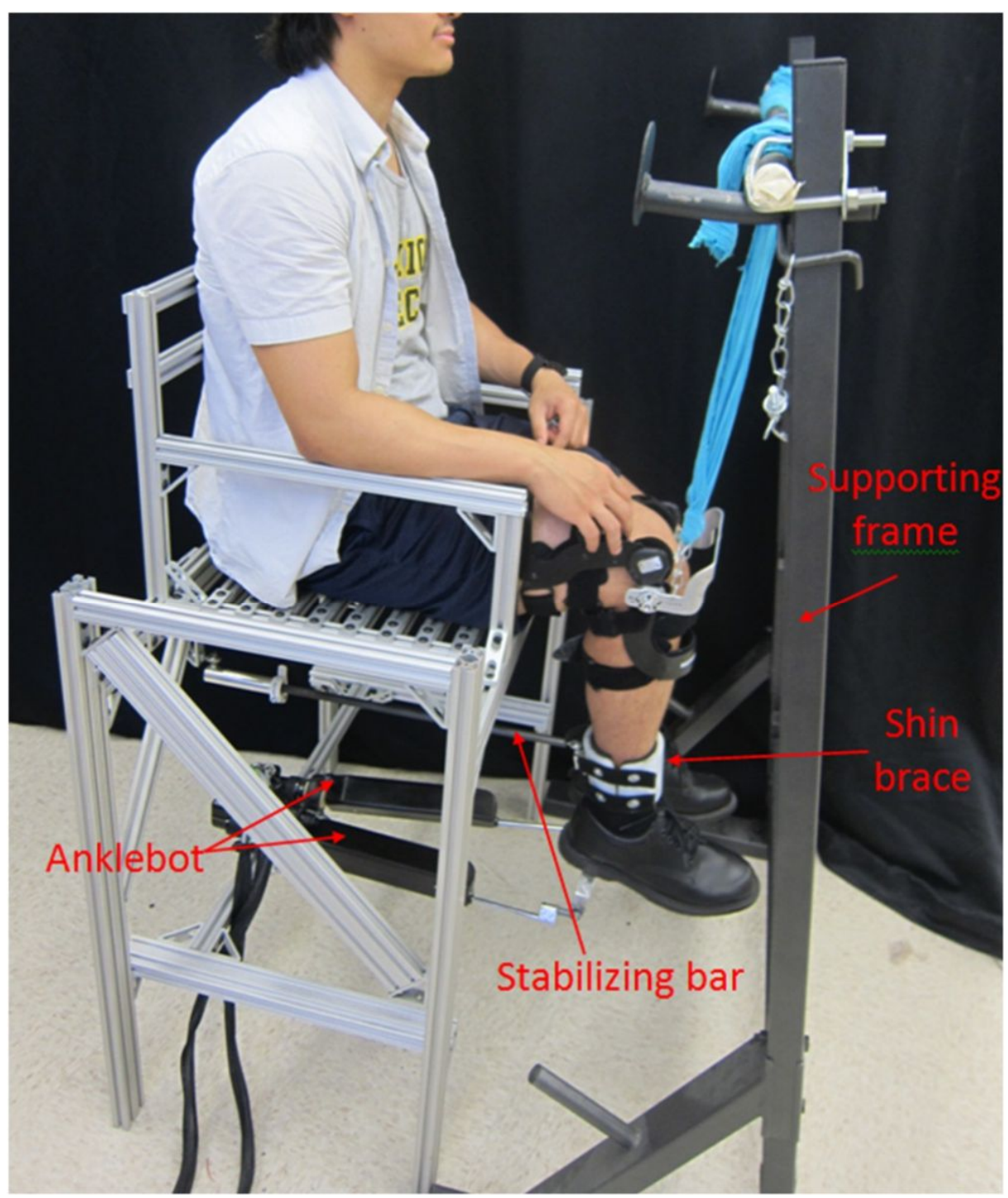

Figure 10.1. Test setup for the estimation of the dynamic mechanical impedance and the quasi-static stiffness of the human lower leg in external-internal rotation direction. 
For the estimation of the mechanical impedance of the lower leg in EI direction, the configuration of the Anklebot was modified, allowing its actuators to apply forces in the transverse plane. As shown in figure 1, a testing chair was fabricated and the Anklebot was securely mounted to the chair horizontally. One end of each actuator was mounted to a horizontal bar that was fixed between the two rear legs of the chair. The moving ends of the actuators were mounted to an aluminum bracket of a modified shoe that was worn by the human subjects. The shoe allowed the force applied by the actuators to rotate the foot in the transverse plane. A stabilizing bar and shin brace were used to constrain the leg from swinging. At one end, the stabilizing bar was mounted underneath the chair using a spherical joint, and at the other end it was connected using a second spherical joint to a semi-cylinder Polyethylene component. The spherical joints allow the shin to rotate in all the anatomical planes, while constraining the translation of the leg in the sagittal plane. A second semi-cylinder Polyethylene component was connected to the first Polyethylene component using two lashing straps forming the shin brace. The lashing straps allow the shin brace to be adjusted to the users' leg dimensions, and the shin brace connects the stabilizing bar to the leg. The internal parts of the shin brace were padded with rubber foam to increase comfort and avoid slippage against the user's skin. A supporting frame was used to support the weight of the Anklebot, shoe, and subject's leg through a knee brace, and to keep the Anklebot actuator at a $90^{\circ}$ angle with respect to the shin in the sagittal plane. At the beginning of each test, the foot was centered at a neutral position using a goniometer. At that position, the knee brace height with respect to the supporting frame was set so the shoe brackets were at the same height as the Anklebot actuators; assuring the Anklebot actuators were at a $90^{\circ}$ angle with respect to the shin in the sagittal plane. Next, the shin brace was attached to the user's shin, and the Anklebot was attached to the shoe. The Anklebot position encoders were set to zero, making this position the reference point for the Anklebot. The actuators of the Anklebot moved the same amount during the tests, but in opposite directions in the sagittal plane. The shin brace 
constrained the shin from translating in the sagittal plane. This way, the lower leg center of rotation remained in the same place. In this configuration, the actuators generated EI rotations of the foot by providing input displacements with identical magnitude and opposite direction with a maximum torque of $15 \mathrm{Nm}$.

Figure 2 shows the schematic of the Anklebot during the experiments. The measured variables were the linear displacement of the left and right actuators of the Anklebot $\left(\mathrm{X}_{\mathrm{L}}\right.$ and $\mathrm{X}_{\mathrm{R}}$, respectively) and the actuation force of each actuator $\left(\mathrm{F}_{\mathrm{L}}\right.$ and $F_{R}$, respectively). To calculate the lower leg impedance and the quasi-static stiffness, both the angles and torques were required. Equations 1 and 2 were used to calculate the angle of the foot $\left(\theta_{E I}\right)$ and applied torques $\left(\tau_{E I}\right)$ in the EI direction based on the kinematics model of the experiment setup shown in figure 2, where D is the distance between the actuators and was set at $0.16 \mathrm{~m}$.

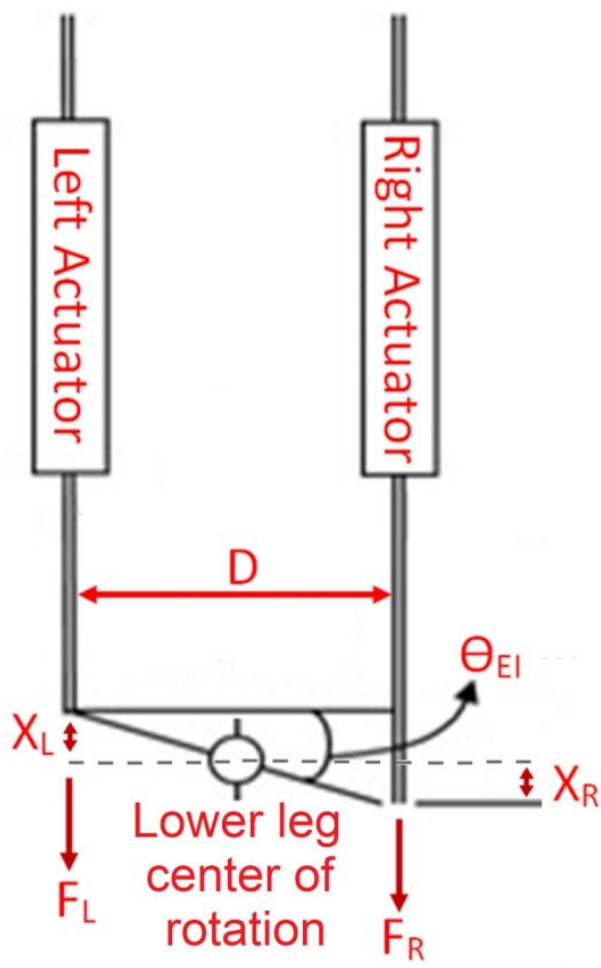

Figure 10.2. Schematics of the Anklebot during the experiments. The Actuators displacements $X_{L}$ and $X_{R}$ are equal in magnitude, and opposite in direction. The lower leg is constrained from translation in the same direction as the displacements $\mathrm{X}_{\mathrm{L}}$ and $\mathrm{X}_{\mathrm{R}}$, thus the lower leg center of rotation does not move. 


$$
\begin{gathered}
\tau_{E I}=\frac{\left(F_{R}-F_{L}\right) \times D}{2} \\
\theta_{E I}=\arctan \left(\frac{X_{L}-X_{R}}{D}\right)
\end{gathered}
$$

\subsubsection{DYNAMIC IMPEDANCE ESTIMATION}

During the experiments, the subjects were instructed to remain relaxed with no muscle contraction. The Anklebot was set to generate pseudo-random voltage inputs with a bandwidth of $100 \mathrm{~Hz}$. The voltage inputs to the actuators were similar in magnitude but with opposite signs, generating lower leg rotations in EI. The ankle range of motion in EI during straight walking at normal speed is near 0.26 radians (5). The perturbations were set to generate the angular displacement of the lower leg with a root-mean-squared (rms) value of 0.065 radians to assure the resulting angular displacements were within a linear range of motion. The Anklebot applied the perturbations for 70 seconds and recorded the data for the applied force and displacement of the actuators at $200 \mathrm{~Hz}$. The first 5 seconds of the recorded data were discarded to remove the effects of any transient interaction dynamics and the initial adaptation of the participants. Next, the angles of rotation of the lower leg and the applied torques were calculated as described in equations 1 and 2.

The Anklebot generated random torque inputs to the foot, which resulted in the rotation of the lower leg in the transverse plane. This system is properly defined as a mechanical admittance that admits torque inputs $(\tau)$ and generates motion output ( $\theta$ ). Linear dynamics were assumed based on small angular displacements of the

applied perturbations ( $0.065 \mathrm{rad} \mathrm{rms})$ with respect to the range of motion of the ankle in the EI (near 0.26 radians) during normal walking (5). Assuming linear dynamics, the admittance $Y$ (as a function of frequency $f$ ), is the transfer function:

$$
\theta=Y(f) \tau
$$

The impedance $Z(f)$ is defined as the inverse of the admittance: 
$Z(f)=Y^{-1}(f)$

In the experiments, the impedance function correlates the input angles to the output torques in EI:

$$
\tau_{E I}=Z(f) \theta_{E I}
$$

During the experiments, a proportional controller with gain $\mathrm{K}=10 \mathrm{Nm}$ was added to the Anklebot controller to hold the foot near its central position and avoid its drift. This value was determined by trial and error to properly hold the foot in its central position for all the users during the experiments. Therefore, the total measured torque $\tau$ is the sum of the proportional controller torque and the human torque:

$$
\tau=k\left(\theta_{o}-\theta_{E I}\right)+\tau_{E I}
$$

where $\theta_{o}$ is the lower leg angle in the neutral position. In the beginning of each test, the foot is centered and the encoders are set to zero, resulting in the neutral position $\theta_{o}$ to be always zero. Combining and simplifying equation 3 and 4 results in:

$$
\tau(f)=(Z(f)+k) \theta_{E I}(f)
$$

Solving for $Z(f)$ in equation 5, yields:

$$
Z(f)=\frac{\tau(f)}{\theta_{E I}(f)}-k
$$

where $\tau(f)$ and $\theta_{E I}(f)$ are the torque and angle measurements from the experiment. To calculate the impedance function, the Matlab's ${ }^{\circledR}$ built in function tfestimate was used. The function tfestimate, finds a transfer function based on the quotient of the cross power spectral density of the torques and angles and the auto power spectral density of the torques. The tfestimate was used with a Hamming window of 512 samples, $50 \%$ overlap, and evaluated with a fast Fourier transform 
length of 1024 samples, resulting in a spectral resolution of $0.19 \mathrm{~Hz}$. The coherence between the input angle and output torque was calculated with the Matlab's ${ }^{\circledR}$ function mscohere with the same parameters as the tfestimate function. The mscohere is a function of frequency with values between 0 and 1 that indicates how well the input correlates to the output at each frequency. The coherence is a function of the power spectral density of the angles, the power spectral density of the torques, and the cross power spectral density of the angles and torques. A final step was required to separate the Anklebot dynamics from the subject's lower leg dynamics. The Anklebot, shoe, and lower leg share the same motion, while the torque measurement is the sum of the torques required to move all of them together. As a result, the estimated dynamic impedance in equation (6) is the sum of the impedances of the lower leg, shoe, and Anklebot. A similar experiment with no human participation was conducted to estimate the impedance of the Anklebot and shoe together. The human impedance $Z_{\text {ankle }}$ is the difference between the estimated impedance functions resulted from the two experiments with the human participants $\left(Z_{\text {lower leg }+ \text { Anklebot }+ \text { shoe }}\right)$ and without the human participants $\left(Z_{\text {Anklebot }+ \text { shoe }}\right)$ :

$\mathrm{Z}_{\text {ankle }}=\mathrm{Z}_{\text {lower leg }+ \text { Anklebot }+ \text { shoe }}-\mathrm{Z}_{\text {Anklebot }+ \text { shoe }}$

\subsubsection{QUASI-STATIC STIFFness Measurement}

The experiments for the estimation of the quasi-static stiffness were performed with the same setup used for the estimation of the dynamic impedance of the lower leg. The quasi-static experiments were performed to validate the results obtained from the impedance estimation experiments. During the quasi-static experiments, the Anklebot was operated in a position control mode with a stiffness of 2177 $\mathrm{Nm} / \mathrm{rad}$ and damping of $100 \mathrm{Nms} / \mathrm{rad}$. These values were found experimentally to generate smooth operation of the Anklebot. Each experiment consisted of rotating the lower leg for 0.4 radians (from the central position) in the internal direction followed by a 0.4 radians rotation in the external direction and back to the central 
position with a constant speed of $0.4 \mathrm{rad} / \mathrm{s}$. This cycle was repeated 10 times without any pause. Below the break frequencies of the estimated dynamic impedance, the visco-elastic elements play the dominant role. The quasi-static experiment is focused on the dynamics of the lower leg in very low frequencies, thus the speed of the quasi-static experiments should be low. However, very low speed would require long experimental time and caused nonlinearities due to stiction in the Anklebot actuators. The loading speed of $0.4 \mathrm{rad} / \mathrm{s}$ was selected experimentally, as it is was found to minimize the effects of the Anklebot's actuator stiction for a smooth operation. The loading speed of $0.4 \mathrm{rad} / \mathrm{s}$ is equivalent to $0.06 \mathrm{~Hz}$, or about $1.74 \%$ of the break frequency (which was found to be $4.4 \pm 0.22 \mathrm{~Hz}$, as it will be described later in the paper).

The recorded angles and torques were filtered with a low pass filter with a cutoff frequency of $1 \mathrm{~Hz}$. For each subject, the lower leg crossed the reference angle (0 degrees) 10 times. To estimate the slopes of each segment (the quasi-static stiffness of the lower leg in $\mathrm{Nm} / \mathrm{rad}$ ) a second order polynomial was fit to each segment in a least square sense. The 10 segments obtained from each test subject were averaged to obtain a representative quasi-static stiffness value for that subject.

\subsection{RESULTS}

\subsubsection{RESULTS FOR DYNAMIC IMPEDANCE}

Figure 3 shows the average dynamic impedance of the lower leg across the subjects as a frequency response plot within the frequency range of up to $30 \mathrm{~Hz}$. The top plot shows the magnitude and the bottom plot shows the phase of the dynamic impedance. The average break frequency, where the phase plot crosses $90^{\circ}$, was found to be $4.4 \pm 0.22 \mathrm{~Hz}$. The average magnitude plot shows a slope of $46.6 \pm 1.5$ $\mathrm{dB} /$ decade beyond the break frequency. The average magnitude below the break frequency was $6.0 \pm 0.85 \mathrm{Nm} / \mathrm{rad}(15.6 \pm 1.4 \mathrm{~dB})$. Figure 4 shows the coherence plot for the dynamic impedance function. The average coherence in the 0 to $30 \mathrm{~Hz}$ range 
was $0.92 \pm 0.004$, with a minimum of 0.85 at $0.89 \mathrm{~Hz}$. The high value of the coherence validates the assumption of the linearity of the dynamic impedance within this frequency range and the conditions of the experiment.

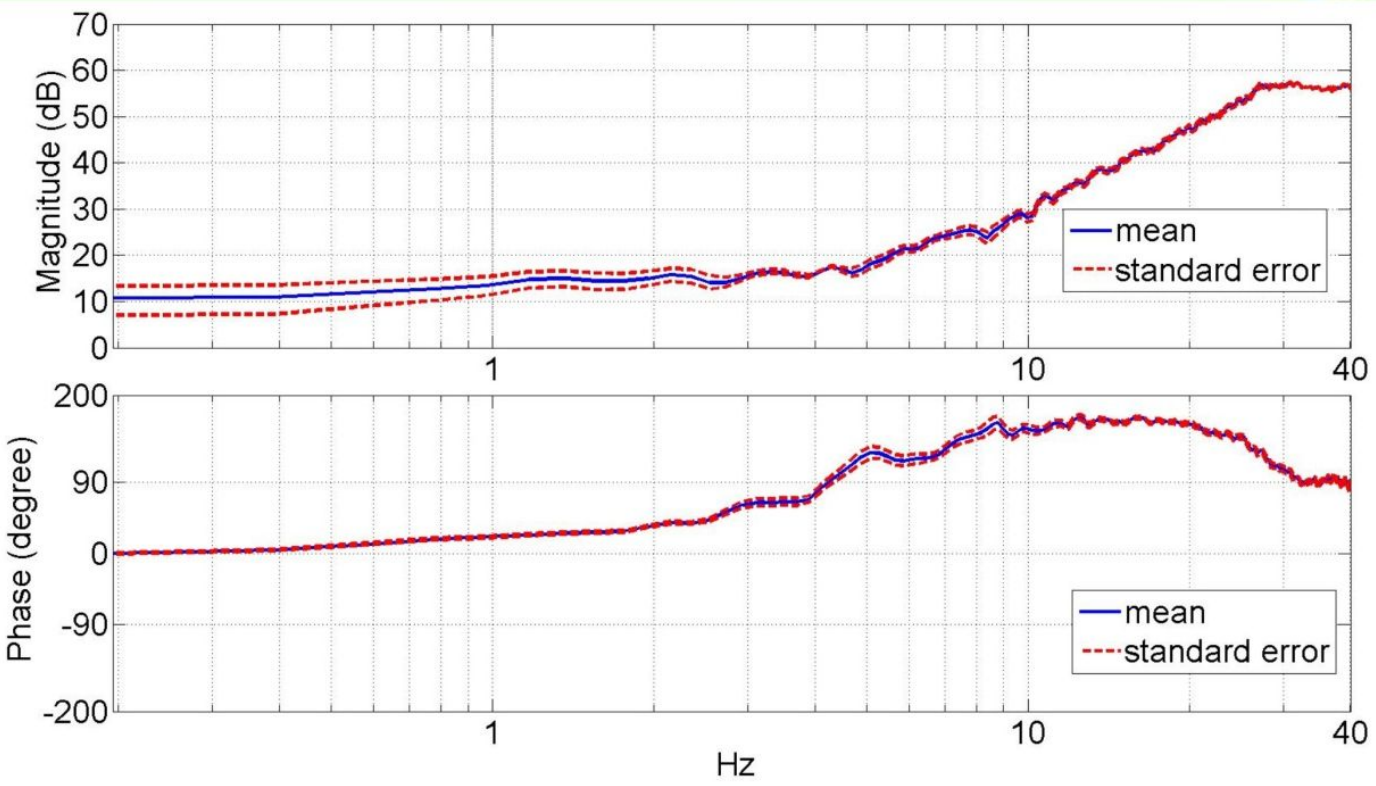

Figure 10.3. Average magnitude and phase plots of the lower leg dynamic mechanical impedance in the external-internal rotation direction.

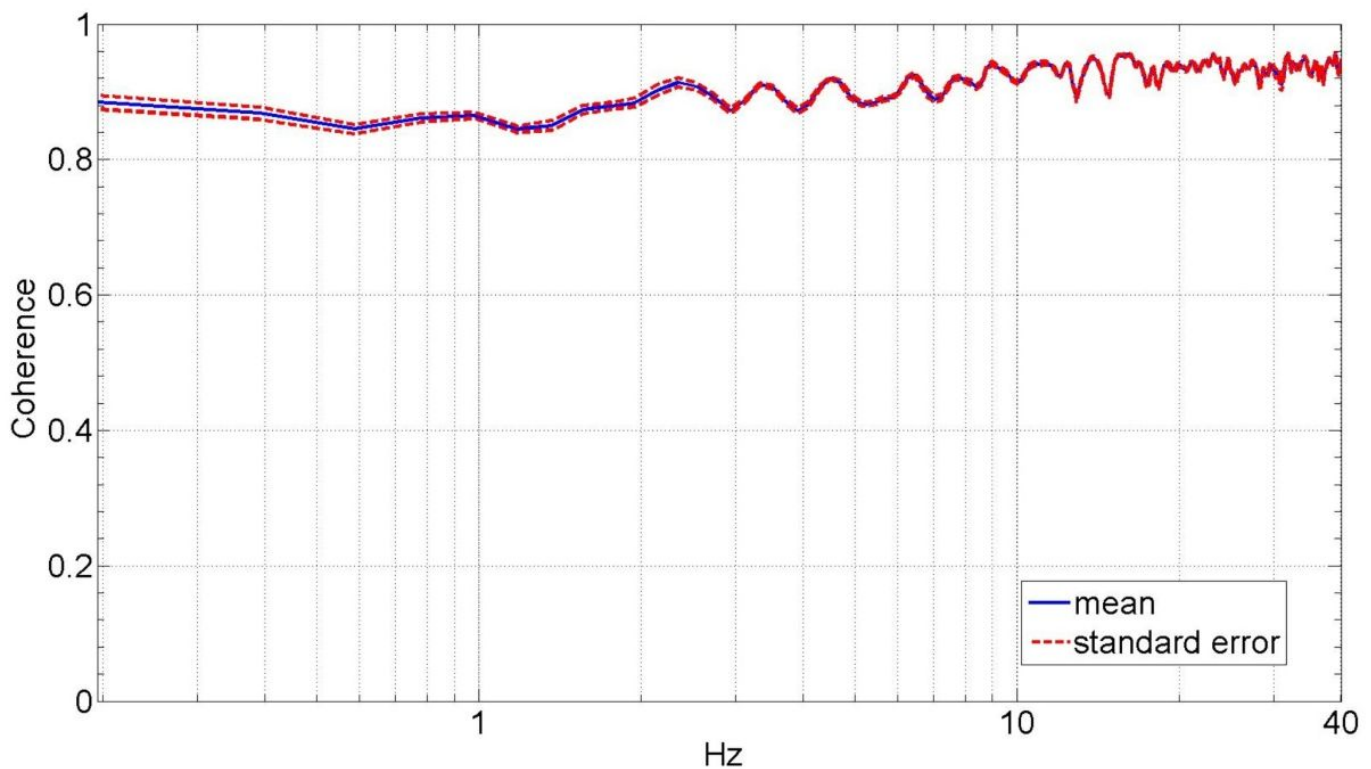

Figure 10.4. Average coherence plot of the lower leg dynamic mechanical impedance in the external-internal rotation direction. 
At very low frequencies $(0-1 \mathrm{~Hz})$, the magnitude plot of the dynamic impedance provides information about the quasi-static stiffness of the lower leg. The quasistatic stiffness magnitude for each test subject averaged over 0 to $1 \mathrm{~Hz}$ is shown in Table 10.1. The average quasi-static stiffness magnitude across the human subjects was $4.90 \mathrm{Nm} / \mathrm{rad}(13.8 \mathrm{~dB})$, with a standard error of $0.74 \mathrm{Nm} / \mathrm{rad}(2.6 \mathrm{~dB})$. A second order system was fit to the data using the MATLAB ${ }^{\circledR}$ function $t$ fest for the impedance results of each subject. The tfest function estimates a transfer function from frequency domain data using the prediction error minimization approach to estimate the transfer function's coefficients. From the second order model, the inertia, damping, and stiffness of each subject's lower leg was estimated as shown in Table 10.2. The average stiffness from the transfer functions was $4.66 \mathrm{Nm} / \mathrm{rad}$ $(13.37 \mathrm{~dB})$ with a standard error of $0.70 \mathrm{Nm} / \mathrm{rad}(3.01 \mathrm{~dB})$. The average damping was $2.25 \pm 0.43 \mathrm{Nm} /(\mathrm{rad} / \mathrm{s})$ and the average moment of inertia was $0.37 \pm 0.03 \mathrm{~kg} . \mathrm{m}^{2}$. The average dynamic impedance transfer function was determined as $Z(s)=0.37 /\left(s^{2}+6.08 s+12.6\right)$.

Table 10.1. Quasi-static stiffness estimated from the averaged impedance in the $0-1$ $\mathrm{Hz}$ range of the participants' lower leg in external-internal direction averaged in the $0-1 \mathrm{~Hz}$.

\begin{tabular}{|c|c|}
\hline Subject number & $\begin{array}{c}\text { Average impedance } \\
\text { magnitude in } \\
\text { 0-1Hz (Nm/rad) }\end{array}$ \\
\hline 1 & 7.82 \\
\hline 2 & 4.51 \\
\hline 3 & 5.64 \\
\hline 4 & 3.52 \\
\hline 5 & 3.99 \\
\hline 6 & 3.03 \\
\hline 7 & 4.86 \\
\hline 8 & 3.07 \\
\hline 9 & 2.63 \\
\hline 10 & 9.95 \\
\hline Mean & 4.90 \\
\hline Standard error & 0.74 \\
\hline
\end{tabular}


Table 10.2. Inertia, damping, and stiffness estimated from the dynamic impedance magnitude and phase of the participants' lower leg in external-internal direction in the $0-30 \mathrm{~Hz}$ range.

\begin{tabular}{|c|c|c|c|}
\hline Subject number & $\begin{array}{c}\text { Estimated } \\
\text { Stiffness } \\
(\mathrm{Nm} / \mathrm{rad})\end{array}$ & $\begin{array}{c}\text { Estimated } \\
\text { Damping } \\
\mathrm{Nm} /(\mathrm{rad} / \mathrm{s})\end{array}$ & $\begin{array}{c}\text { Estimated } \\
\text { Inertia } \\
\left(\mathrm{kg} \cdot \mathrm{m}^{2}\right)\end{array}$ \\
\hline 1 & 6.85 & 0.95 & 0.30 \\
\hline 2 & 5.39 & 0.80 & 0.26 \\
\hline 3 & 5.73 & 3.54 & 0.44 \\
\hline 4 & 3.38 & 2.14 & 0.33 \\
\hline 5 & 3.87 & 2.23 & 0.43 \\
\hline 6 & 2.72 & 1.83 & 0.32 \\
\hline 7 & 4.74 & 2.44 & 0.52 \\
\hline 8 & 2.96 & 1.93 & 0.35 \\
\hline 9 & 1.80 & 1.19 & 0.25 \\
\hline 10 & 9.15 & 5.40 & 0.51 \\
\hline Mean & 4.66 & 2.25 & 0.37 \\
\hline Standard error & 0.03 & 0.43 & 0.70 \\
\hline
\end{tabular}

\subsubsection{RESULTS FOR QUASI-STATIC STIFFNESS}

The quasi-static impedance of the lower leg in EI was estimated directly for each subject and the results are shown in Table 10.3. The average torques applied to the foot of all the subjects plotted against the corresponding average lower leg angles is shown in figure 5. The average quasi-static stiffness using this method was $5.81 \mathrm{Nm} / \mathrm{rad}$ with a standard error of $0.81 \mathrm{Nm} / \mathrm{rad}$.

To compare the results of the quasi-static stiffness obtained from the two methods, only the data where the lower leg rotations were within \pm 0.065 radians was used. The angular motion in the lower leg within the range of \pm 0.065 radians is near linear when plotted against the estimated torque as shown in figure 5. In figure 5 the slope represents the quasi-static stiffness of the lower leg. Since the lower leg crossed the reference angle (0 degrees) 10 times, the experimental data (both angles and torques) were divided into 10 segments of equal length in the range of \pm 0.065 radians. One-way analysis of variance was used to compare the results of the quasistatic stiffness from direct experiments, the quasi-static stiffness from the average 
impedance magnitude in the $0-1 \mathrm{~Hz}$ range, and the stiffness obtained from the transfer function estimated from the impedance measurement. The p-value was found to be 0.53 showing that the results obtained from the three methods are statistically similar.

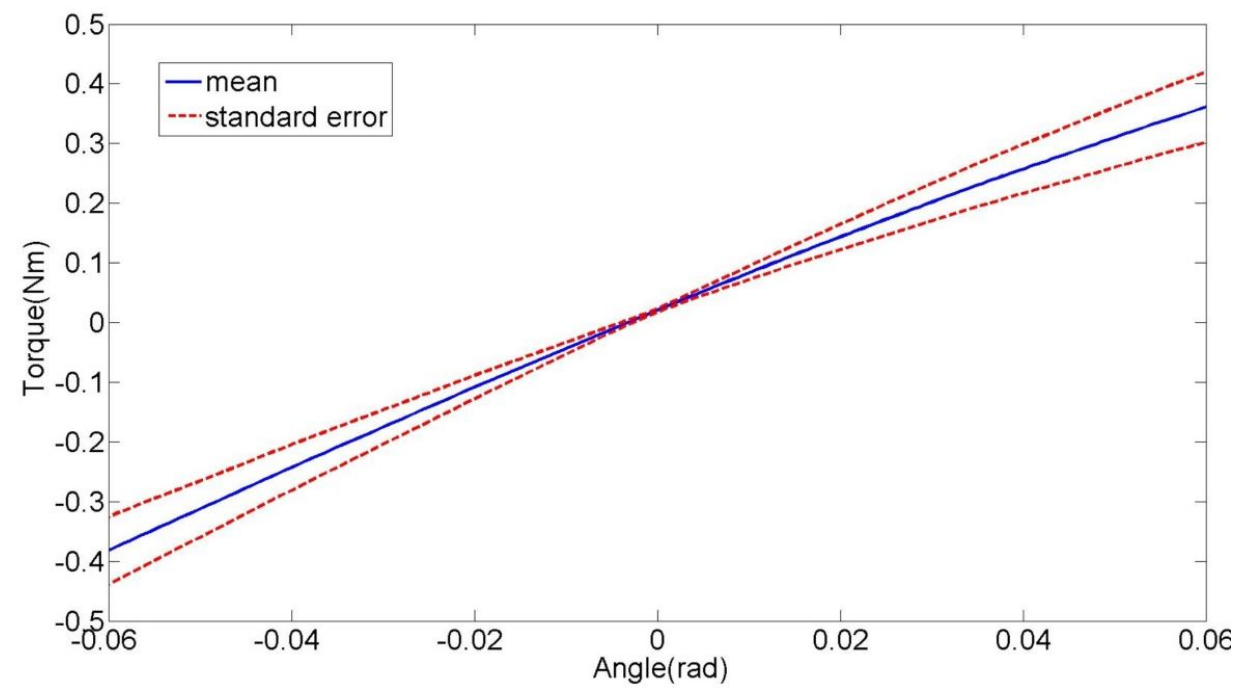

Figure 10.5. Plot of the average lower leg torque vs. lower leg angle during the quasi-static stiffness experiment in external-internal rotation direction. The slope represents the stiffness of the lower leg.

Table 10.3: Quasi-static stiffness of the participants' lower leg from the direct estimation..

\begin{tabular}{|c|c|}
\hline Subject number & $\begin{array}{c}\text { Quasi-static } \\
\text { stiffness } \\
(\mathrm{Nm} / \mathrm{rad})\end{array}$ \\
\hline 1 & 8.73 \\
\hline 2 & 5.23 \\
\hline 3 & 5.43 \\
\hline 4 & 3.52 \\
\hline 5 & 3.28 \\
\hline 6 & 5.21 \\
\hline 7 & 4.74 \\
\hline 8 & 3.63 \\
\hline 9 & 7.07 \\
\hline 10 & 11.29 \\
\hline Mean & 5.81 \\
\hline Standard error & 0.81 \\
\hline
\end{tabular}




\subsection{DISCUSION}

Experimental and analytical methods were presented for the estimation of the mechanical impedance of the human lower leg in the transverse plane. The presented results are bound to the experiment conditions, including suspended lower leg under no load with no muscle contraction with a $90^{\circ}$ bent knee. In this configuration, the rotations of the ankle's talocrural and the subtalar joints are dominant in the recorded dynamics, and any contribution of the tibiofibular joints and keen joint are also measured. Similar to the mechanical impedance of the lower leg in sagittal and frontal planes, the lower leg impedance in the transverse plane showed a behavior close to a second degree function. A second order mechanical impedance is a function of inertia, visco-elastic properties, and stiffness of the muscles and tendons of the lower leg that contribute to the rotation of the lower leg in the transverse plane. While in higher frequencies the effects of the inertia are dominant, in the low frequencies, the visco-elastic properties of the lower leg due to the passive characteristics of the soft tissues are dominant. Visco-elastic elements have near constant stiffness below the break frequency, generating the observed near constant impedance magnitude plot at low frequencies. A break frequency would separate high- and low-frequency regions, and its average was determined to be $4.4 \pm 0.22 \mathrm{~Hz}$ for the populations of this study. The slope of the average magnitude plot was $46.6 \pm 1.5 \mathrm{~dB} /$ decade that is consistent with the results obtained in DP and IE $(50,122)$ with a break frequency of $8 \sim 10 \mathrm{~Hz}$. This showed that the break frequency of the impedance function in EI is lower than DP and IE. The average impedance magnitudes in DP and IE directions in the frequency range of less than $2 \mathrm{~Hz}$ were reported as $12.61 \pm 1.27 \mathrm{Nm} / \mathrm{rad}$ and $7.96 \pm 0.62 \mathrm{Nm} / \mathrm{rad}$, respectively (122). The quasi-static stiffness in EI direction, from the average impedance magnitude in the $0-1 \mathrm{~Hz}$ range, was $4.90 \pm 0.74 \mathrm{Nm} / \mathrm{rad}$, indicating the lower leg is most compliant in the transverse plane. 
For the estimated impedance function, the coherence was close to unity (average of 0.92 ) at all frequencies up to $30 \mathrm{~Hz}$ with a minimum of 0.85 at $0.89 \mathrm{~Hz}$, indicating that lower leg impedance in EI is well characterized by linear models under the given experimental conditions. This coherence is similar to the reported coherence for the estimated ankle mechanical impedance function in DP and IE directions $(50,113,122)$. Additionally, the analysis method requires that the actuator impedance function to be identified using a similar stochastic identification method and subtracted from the impedance of the combined lower leg and actuator. The coherence for the impedance function for the actuator alone was 0.93 , implying a plausible linear behavior of the experiment setup.

Fitting a second order system to the frequency domain data of each subject's impedance estimation, a transfer function was obtained. From the second order model, an average inertia of $4.66 \pm 0.70 \mathrm{Nm} / \mathrm{rad}$, average damping of $2.25 \pm 0.43$ $\mathrm{Nm} /(\mathrm{rad} / \mathrm{s})$, and an average moment of inertia of $0.37 \pm 0.03 \mathrm{~kg} . \mathrm{m}^{2}$ were obtained. All three parameters show similar variance among the subjects. The average estimated stiffness from the transfer function was $0.24 \mathrm{Nm} / \mathrm{rad}$, which was smaller than the average quasi-static stiffness averaged from the impedance measurement in the $0-1 \mathrm{~Hz}$ range, but it was within its standard error limits. The smaller value in the stiffness from the estimated transfer function is expected as the quasi-static stiffness from the impedance measurement takes the effects of damping and inertia within the 0-1 Hz range into account.

The quasi-static stiffness from direct estimation was $5.81 \pm 0.81 \mathrm{Nm} / \mathrm{rad}$ compared to $4.9 \pm 0.74 \mathrm{Nm} / \mathrm{rad}$ for the quasi-static stiffness averaged over the impedance magnitude in the $0-1 \mathrm{~Hz}$ range, and $4.66 \mathrm{Nm} / \mathrm{rad} \pm 0.70$ from the estimated transfer function stiffness. The analysis of variance ANOVA was used to compare the results of the three methods. The p-value was found to be 0.53 , indicating no statistically significant difference between the results. 
The presented results can be used on the evaluation of patients suffering from spasticity due to stroke or patients with multiple sclerosis. It can be used to quantify the level of impairment and monitor the progress during the course of treatment. In addition, the results of the study provides a base for furthering our understanding of the lower leg musculoskeletal dynamics. During walking, the lower leg will be under varying load during the stance phase. Additionally, the lower leg's muscles co-contractions would change the lower leg impedance during the stance phase. The presented experiment and analysis method will be extended to estimate the mechanical impedance of the lower leg with different muscle activation levels, and different knee and ankle angles to define a relationship between muscle activation levels and the mechanical impedance of the lower leg. This may provide design parameters for the development of ankle-foot prostheses with characteristics comparable to the human lower leg. This is particularly important since designing protocols for the estimation of the mechanical impedance of the lower leg or ankle in the transverse plane during walking is challenging. This is evident from the recent studies on estimation of the mechanical impedance of the ankle in the sagittal and frontal planes during stance phase of gait $(58,104,123)$; however, there has not been any study in the transverse plane. The development of state of the art anklefoot prostheses may greatly benefit from the estimation of the time-varying mechanical impedance of the human ankle and lower leg in all three anatomical planes, which will be the focus of future work.

\section{CONCLUSION}

This paper described the protocols and results for the estimation of the dynamic mechanical impedance and the quasi-static stiffness of the human lower leg in the external-internal rotation direction. Under the conditions of this experiment, the participants' lower legs were suspended under no load, with knees bent $90^{\circ}$, and relaxed muscles. In the presented configuration, the recorded lower leg dynamics are due to the rotations of the ankle's talocrural and the subtalar joints, and any 
contribution of the tibiofibular joints and keen joint. For dynamic mechanical impedance estimation, pseudo-random torque perturbations were applied to the foot, causing its movement in the transverse plane. For the quasi-static stiffness estimation, ramp perturbations with a constant velocity of $0.4 \mathrm{rad} / \mathrm{sec}$ were applied to the foot, generating the quasi-static motion of the foot in the transverse plane. The dynamic mechanical impedance of the lower leg was estimated in frequency domain with an average coherence of 0.92 within the frequency range of 0 to $30 \mathrm{~Hz}$ range, implying a linear correlation between the recorded angular displacement and the torques. The mean magnitude of the quasi-static stiffness from the averaged impedance of the lower leg in the $0-1 \mathrm{~Hz}$ range was $4.9 \pm 0.74 \mathrm{Nm} / \mathrm{rad}$. The results of the experiment for direct estimation of the quasi-static stiffness of the lower leg resulted in the means value of $5.8 \pm 0.81 \mathrm{Nm} / \mathrm{rad}$. An analysis of variance determined that the difference in the estimated values for the quasi-static stiffness from the two experiments were not statistically significant. 


\section{1- DESIGN OF A STEERABLE POWERED ANKLE- FOOT PROSTHESIS CAPABLE OF TURNING}

\subsection{ABSTRACT}

This paper describes the design of an ankle-foot robotic prosthesis controllable in the sagittal and frontal planes. The prosthesis was designed to meet mechanical characteristics of the human ankle including power, range of motion, and weight. To transfer the power from the motors and gear boxes to the ankle-foot mechanism, a Bowden cable system was used. The Bowden cable allows for optimal placement of the motors and gear boxes, reducing the metabolic energy required for locomotion. Additionally, it allows flexibility for the customization of the device to amputees with different residual limb sizes. To control the prosthesis, impedance controllers in both sagittal and frontal planes were developed. The impedance controllers used torque feedback from strain gauges installed on the foot. Preliminary evaluation was performed to verify the capability of the prosthesis to track the kinematics of the human ankle in two Degree of Freedom (DOF) and the ability of the prosthesis to modulate the impedance of the ankle. Moreover, the system was characterized for describing the relationship between the gains of the impedance controllers to the actual stiffness of the ankle. In Dorsiflexion-Plantarflexion (DP) the stiffness ranged from $282 \mathrm{Nm} / \mathrm{rad}$ to $22.5 \mathrm{Nm} / \mathrm{rad}$. In inversion-Eversion (IE), the stiffness ranged from $36 \mathrm{Nm} / \mathrm{rad}$ to $6 \mathrm{Nm} / \mathrm{rad}$. The prosthesis is capable of properly mimicking human ankle kinematics and changing its mechanical impedance in two DOF in real time with a range of stiffness sufficient for normal human walking.

\subsection{INTRODUCTION}

Walking requires activation of the leg muscles to control the ground reaction forces while modulating the mechanical impedance of the lower leg, especially at the ankle. The ankle is the first major joint to transfer the ground reaction torques to 
the body and plays a major role in locomotion. The lack of propulsion in bellow knee amputees who use passive prostheses require $20-30 \%$ more energy than nonamputees when walking at the same speed, and their preferred speed of gait is 30$40 \%$ slower than unimpaired individuals $(39,40)$. Additionally, bellow knee amputees requires different gait strategies compared to the non-amputees that results in secondary injuries due to overuse or misuse of their healthy joints, and cardiovascular diseases due to the lack of mobility (7). Currently there are nearly 2 million amputees in the United States (2). The main causes of amputations are vascular diseases (54\%), which includes diabetes and peripheral arterial disease, and trauma (45\%) (2). The mortality rate of amputees due to vascular diseases is $50 \%$ within the first 5 years. This 5 year mortality rate is higher than breast cancer, colon cancer, and prostate cancer (3). Lower limb accounts for $97 \%$ of all amputations due to vascular diseases (4), resulting in near 1 million of lower limb amputees in the United States. A powered ankle-foot prosthesis with anthropomorphic characteristics may improve the metabolic cost in bellow knee amputees bringing it closer to the values found for unimpaired subjects. Such prosthesis can increase mobility, comfort, and agility. These improvements further translate into increase in activity levels, improvement on obesity and cardiovascular diseases, and overall improvement in the quality of life of amputees.

Current state-of-the-art powered ankle-foot prostheses are designed to improve sagittal plane mobility by focusing on control of the ankle in one DOF; that is, they seek to regulate dorsiflexion and plantarflexion of the powered ankle $(27,69,70)$. However, this design strategy does not fully incorporate the walking mechanism in unimpaired individuals because even during walking on a straight path, the human ankle functions in both the sagittal and frontal planes. Additionally, activities of daily leaving (ADLs) includes other gait scenarios that require agility and maneuverability, such as turning, traversing slopes, and adapting to uneven terrain profiles. Some ADLs require $8 \%$ to $50 \%$ of turning steps depending on the activity (1). Additionally, significant changes in the ankle kinetics and kinematics in the 
ankle Inversion-Eversion (IE) direction are observed when comparing straight walking and sidestep cutting $(5,35)$. At weight acceptance, the ankle torque in IE increased from $0.04 \pm 0.01 \mathrm{Nm} / \mathrm{rad}$ in eversion during the straight walk to $0.11 \pm 0.02$ $\mathrm{Nm} / \mathrm{rad}$ in eversion during the sidestep cutting. The angles changed from $1^{\circ} \pm 0.8^{\circ}$ in eversion during the straight walk to $4^{\circ} \pm 0.6$ in inversion during the weight acceptance of the sidestep cutting. This result shows that although the ankle torques increased in the eversion direction the ankle angles increased in the inversion direction when comparing straight walking to sidestep cutting. Larger changes were observed at mid-stance phase of gait where the ankle torque in IE increased from $0.19 \pm 0.02 \mathrm{Nm} / \mathrm{rad}$ in eversion during the straight walk to $0.29 \pm 0.03 \mathrm{Nm} / \mathrm{rad}$ in eversion during the sidestep cutting, and the angles changed from $5^{\circ} \pm 0.3^{\circ}$ in eversion during the straight walk to $4^{\circ} \pm 0.6$ in inversion during the sidestep cutting. This result shows that ankle torques are required in the frontal plane during straight walk, and even larger torques are required for turning. This context suggests that the next advance in lower extremity prosthetic devices is to extend their design and control to the frontal plane.

The authors developed a proof-of-concept 2-DOF cable-driven robotic anklefoot prosthesis $(117,118)$. While the authors' previous design used actuators fixed to the pylon, the new design presented here uses Bowden cables; therefore, it allows the placement of the actuators at a location preferred by the user or to improve its performance. Cable driven systems have been used in the design of exoskeletons and assistive robots; however, this is the first lower extremity robotic prosthesis with such design. A cable-driven system mimics the musculoskeletal system, where muscles pull tendons to generate motion. The cable-driven prosthesis allow flexibility in the positioning of the motors when they do not need to be at the vicinity of the joints, allowing for low profile designs with tailored size, weight, and inertia. It has been reported that a mass at the foot increases the user metabolic cost by $8-9 \%$ per one kilogram of the mass during walking, while the mass carried at the waist increases the metabolic cost by only $1-2 \%$ (124). Hence, reducing the mass at 
the ankle-foot extremity by placing the actuators, battery, and the electronics away from the distal part of the leg would reduce the metabolic cost of gait.

Two interacting physical systems behave either as an impedance (i.e. accepts external motion inputs and generates force outputs) or as an admittance (i.e. accepts external force inputs and generates motion outputs) (102). During gait, the ankle joint interacts with ground generating the appropriate torques to move the body. The impedance is defined as the evoked force due to an input motion perturbation. In the case of the ankle, the evoked torque is due to an angular displacement of the ankle. The change in ankle torques and angles from weight acceptance to mid stance suggests the ankle presents a time-varying impedance in the sagittal plane $(5,116)$. Additionally, the changes from straight walk to sidestep cutting suggests that the ankle presents a task-dependent impedance. Other studies have shown the ankle time-varying impedance characteristic. Rouse et al. evaluate the ankle impedance in the sagittal plane during the foot-flat sub-phase of stance (49), and Lee et al. studied the ankle during pre-swing, swing, and early stance of the gait in both frontal and sagittal planes (58). These studies also showed a time-varying behavior of the human ankle in both DP and IE suggesting a robotic prosthesis needs to be able to change its impedance considerably during the stance phase of gait.

This motivated the development of powered ankle-foot prostheses controllable in both the frontal and sagittal planes with variable impedance in both axes. In this paper, we described in detail the use of Bowden cables in the actuation system of the developed ankle-foot prosthesis, the ability of the mechanism to mimic the human kinematics during turning, the estimation of ground reaction torques in two DOF in the sagittal and frontal planes, and the development and testing of impedance controllers in both the frontal and sagittal planes. 


\subsection{DESIGN DETAIL OF THE 2-DOF ANKLE FOOT PROSTHESIS}

A prototype cable-driven ankle-foot prosthesis (Figs. 11.1 and 11.2) with two controllable DOF in both frontal and sagittal planes was developed. For an average able-bodied human weighing $80 \mathrm{~kg}$, the required energy and torque are $36 \mathrm{~J}$ (250 watts peak power) (69) and $140 \mathrm{Nm}$ (32), respectively at each step during straight walk. These amounts are $35 \%$ higher for an individual with a passive transtibial prosthesis $(32,69)$. The developed ankle-foot prosthesis was estimated to have $40 \%$ losses, resulting in an anticipated peak power consumption of 470 watts, energy consumption of $68 \mathrm{~J}$, and a peak torque of $264 \mathrm{Nm}$ in the sagittal plane.

The design of the prototype was based on the human power requirements. The prototype uses two brushless motors and planetary gearheads (A). Each motor (Maxon EC-4pole part number 305014) is rated at 200 watts (for a total of 400 watts), and the gear boxes have a reduction ratio of 81:1 (Maxon gearhead GP 42 part number 203124). The motors are powered using two Maxon motor controllers ((J) part number 438725) connected to a 22.2 lithium polymer battery. Two cable drums (B) transfer the required torque to the ankle through four Bowden cables (C). At a bracket (D) mounted to the pylon (E), the Bowden cables are connected to the spherical joints $(\mathrm{K})$ to allow self alignment of the Bowden cables. The bracket on the pylon can be lowered near the ankle and the pylon can also be shortened to accommodate the requirements of amputees with long residual limbs. A universal joint $(\mathrm{F})$ connects the pylon to an elastic carbon-fiber plate $(\mathrm{G})$ that is connected to a commercially available foot $((\mathrm{H})$ Össur Flex-foot). The carbon fiber plate acts as a spring in series with the cables. It is designed to have adequate stiffness to transfer the required torque while being flexible enough to store and releases energy to assure the cables are always under tension.

The cables needs to always be in tension to facilitate proper control over the foot, resulting in the carbon fiber plate to always be under a bending moment. In the rear side of the carbon-fiber plate, the cables are mounted at both sides of the 
longitudinal axis of the foot. At the front side, the cables are passed through a pulley (I). The passive components of the ankle-foot prosthesis (Fig. 11.2) weigh $1.13 \mathrm{~kg}$ and the motor and transmission (including the gear boxes, cable drums, and Bowden cables) weight $2.2 \mathrm{~kg}$.

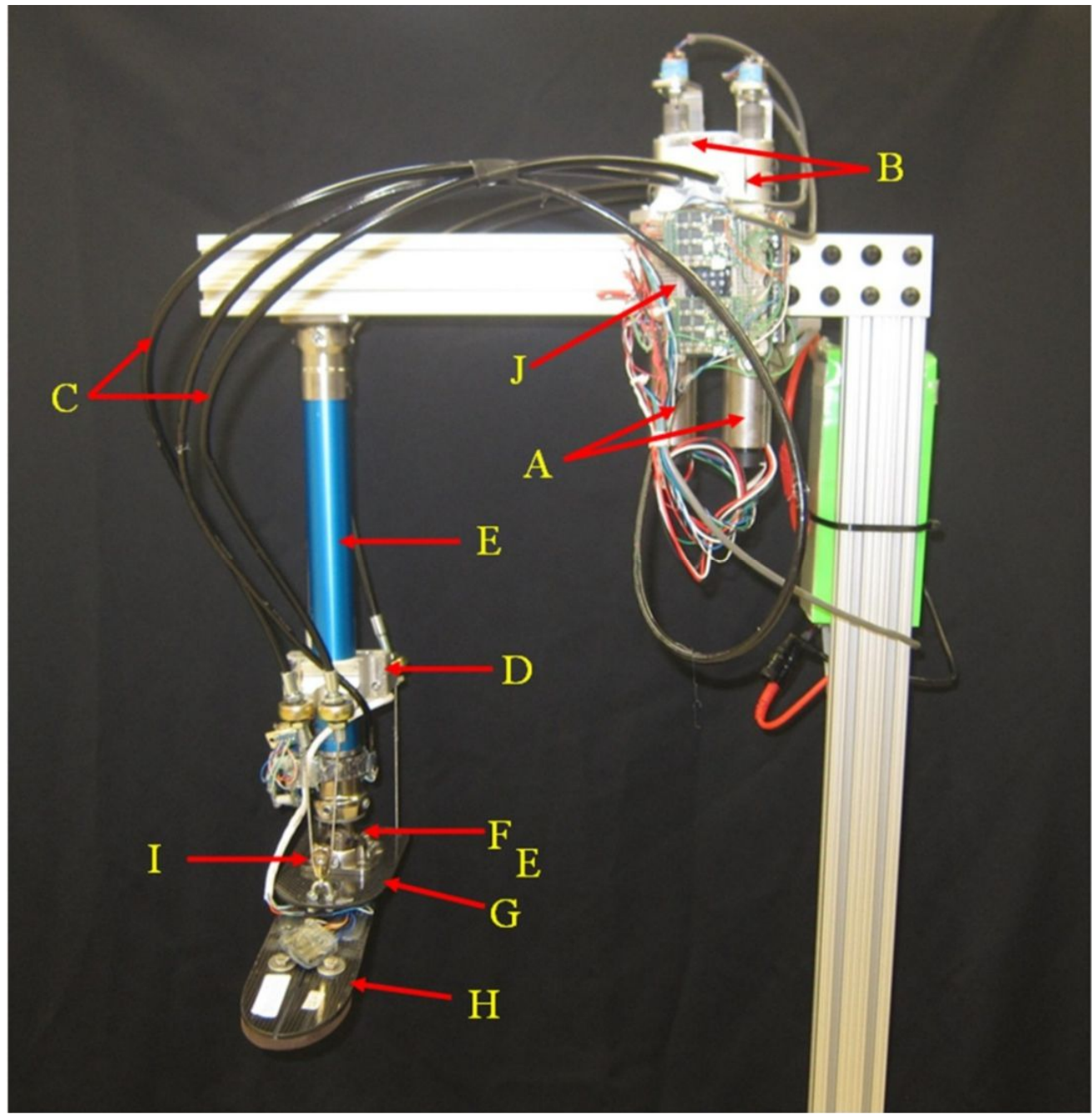

Figure 11.1. 2-DOF cable driven ankle-foot prosthesis.

To control the prosthesis in both the frontal and sagittal planes, position controllers were developed. The presented design relies on the fact that three points are sufficient to define a plane in the space. The robot has control over two DOF and uses two motors to generate its motion. The sum of motor toques while rotating 
in opposite directions generated motion in DP and the difference in torques while rotating in the same direction generates the motion in IE. A combination of IE and DP motion is obtained with different input signals to each motor in both direction and magnitude. This implies that the prosthesis requires two independent controllers, one for each motor. The position controllers use position plus derivative (PD) controllers as shown in Fig. 11.3.

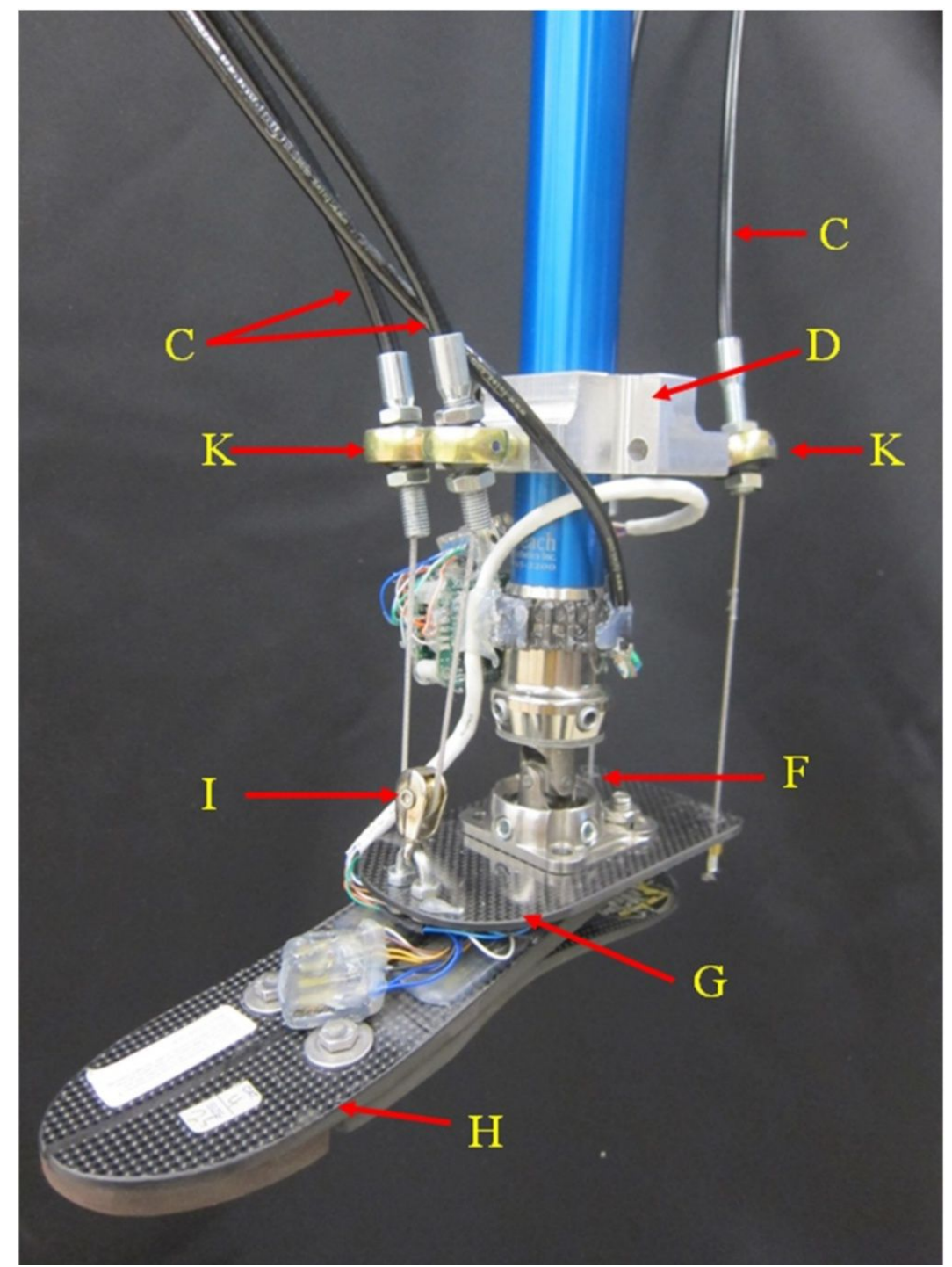

Figure 11.2. Detail of the passive components on the 2-DOF cable driven ankle-foot prosthesis. 


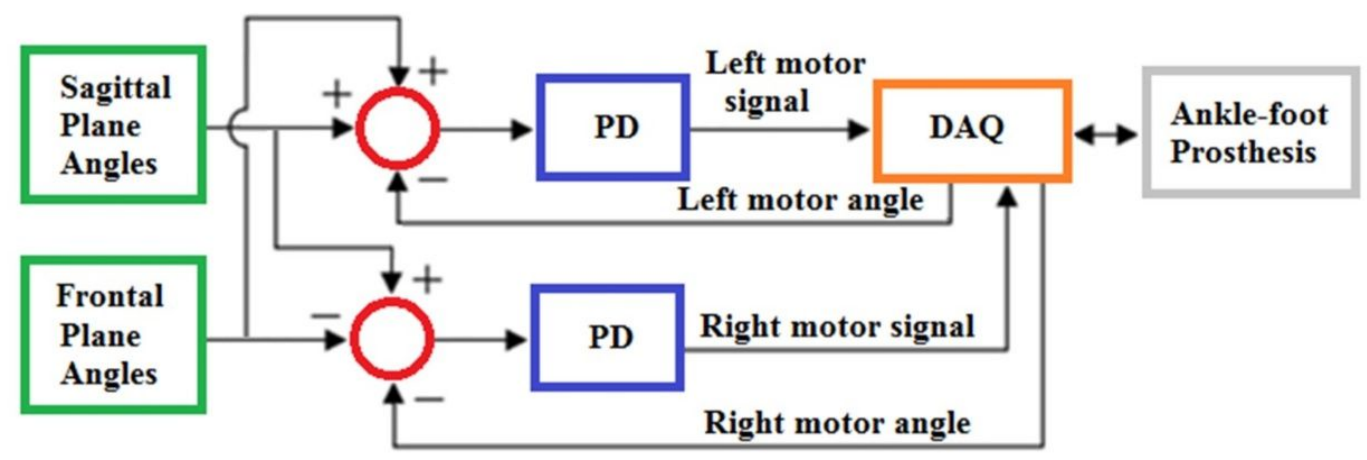

Figure 11.3. Block diagram of the position controllers.

\subsection{HUMAN ANKLE KINEMATICS TRACKING}

To evaluate the developed controller, the pre-recorded data of the kinematics of the ankle of a human subject during a step turn was used as the input. The ankle rotations were recorded using a motion capture camera system (OptiTrack Prime $17 \mathrm{~W})$. The controllers used the pre-recorded human motion to adjust the neutral position of the ankle and position feedback from quadrature encoders mounted on each motor to estimate the appropriate motor inputs using PD controllers. For one of the motor controllers, the input reference angle is the sum of the sagittal and frontal planes angles. For the other motor controller, the reference angle is the difference between the sagittal and frontal planes angles.

The ankle-foot prosthesis was capable of mimicking the recorded human ankle motion in both frontal and sagittal planes. Fig. 11.4 shows the output trajectories that followed closely to the human ankle rotations, indicating a plausible kinematics design. The system showed a $40 \mathrm{~ms}$ delay between the input and output, which was removed for ease of comparison. 

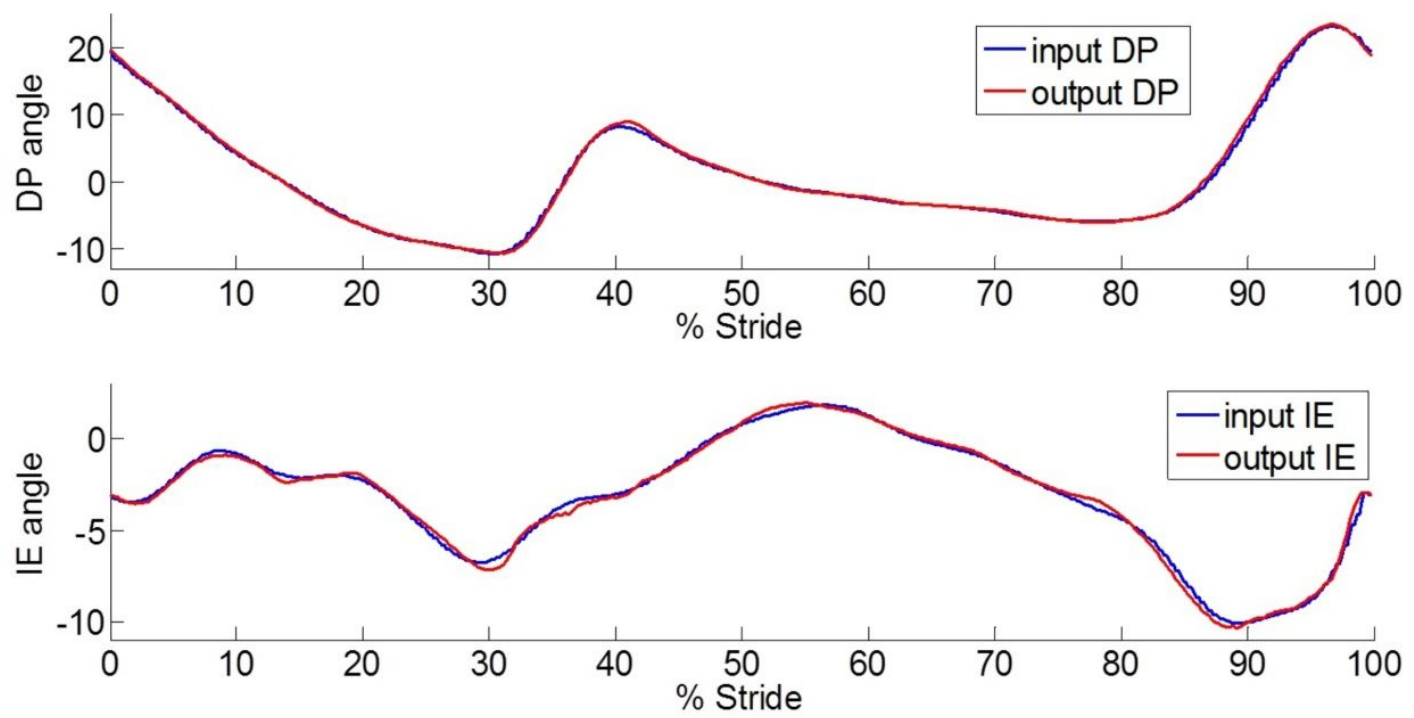

Figure 11.4. Plot of the ankle-foot prosthesis trajectories in dorsiflexionplantarflexion and inversion-eversion directions that closely follow the human ankle rotations during a step turn stance phase and the prior swing period. The input has a time shift of 40 milliseconds to compensate for the system delay.

\subsection{ANKLE TORQUE FEEDBACK FOR IMPEDANCE CONTROLLERS}

Strain gauges were used for torque estimation in two DOF in the prosthesis. The Össur Flex-foot was chosen since it is composed of two leaf springs, one on each side of the foot in the sagittal plane (Fig. 11.5). This was desirable, as the load on the left and right sides of the foot can be measured independently using strain gauges in a Whetstone Bridges configuration (one Whetstone Bridge in each side of the foot). The sum of strains is proportional to the load in DP and the difference in strains is proportional to the load in IE. The sign of the sum of the measured strains in DP indicates if the torque is generating dorsiflexion or plantarflexion. In IE, a simple logic in the controller is used to identify the direction of the torques. The presence of combined inversion and dorsiflexion or combined eversion and plantarflexion torques generate a positive IE signals. On the other hand combined eversion and dorsiflexion or combined inversions and plantarflexion torques 
generate negative signals in IE. Following this logic, the controller first checks the direction of the torque in DP, and then, if the IE signal is positive or negative estimates if the signal is due to an inversion torque or an eversion torque.

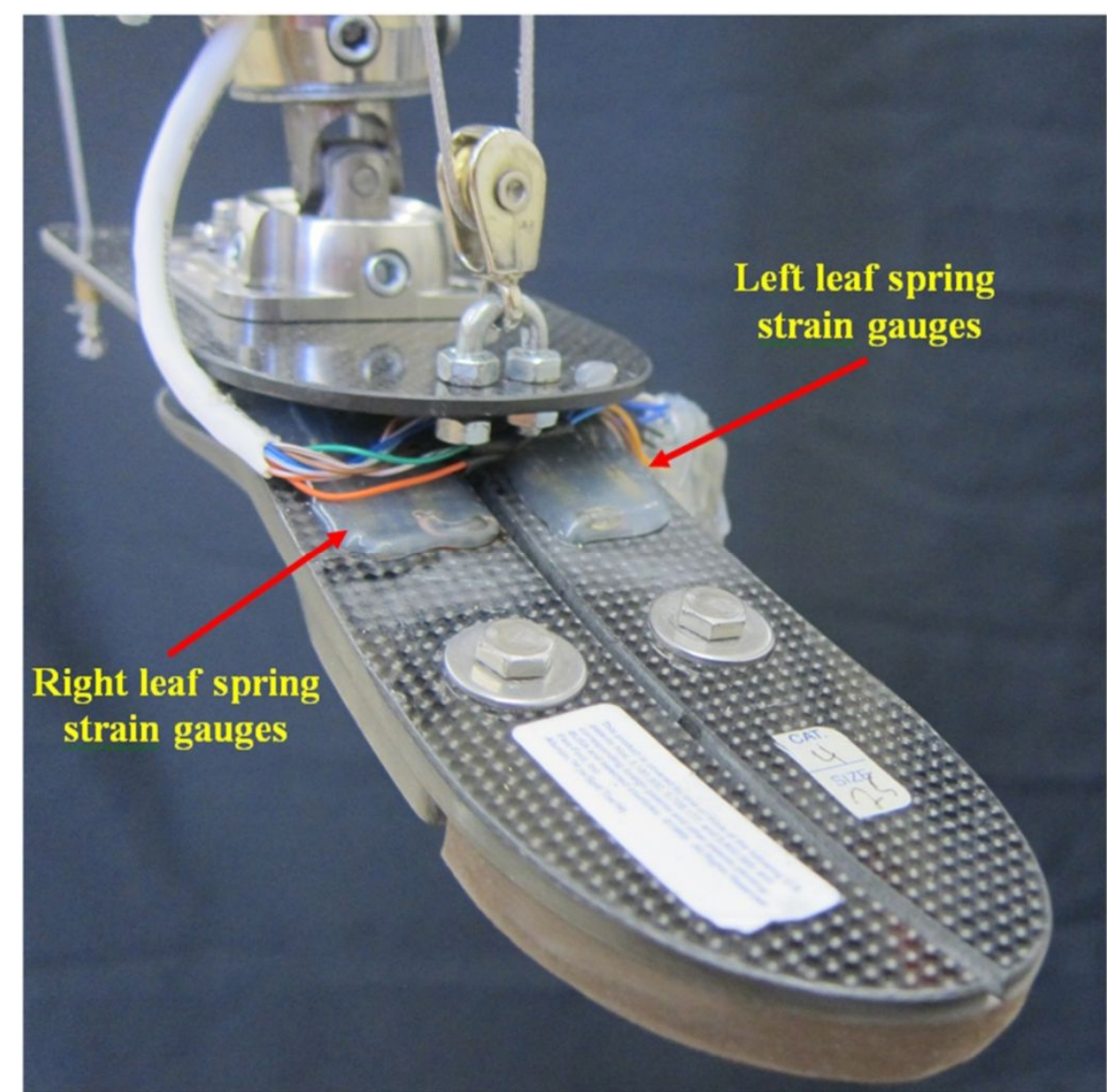

Figure 11.5. The strain gauges installed on the foot for torque estimation in both DP and IE on the foot (Össur Flex-foot).

To correlate the strain measurements to the applied torque to the foot, calibration was conducted using a force plate. The foot was loaded at 4 different points, generating isolated torques in inversion, eversion, plantarflexion, and dorsiflexion, respectively. The force applied to the foot, the strain gauge measurement at each Whetstone Bridge $\left(\mathrm{S}_{\mathrm{L}}\right.$ and $\mathrm{S}_{\mathrm{R}}$ for the left and right bridges, respectively), and the distance from the points where the force was applied to the 
ankle center of rotation were measured. Based on these parameters, the gains $K_{P}$, $\mathrm{K}_{\mathrm{D}}, \mathrm{K}_{\mathrm{I}}$, and $\mathrm{K}_{\mathrm{E}}$ were estimated to calculate the torques in terms of the strain measurements as seen in equations 1-4:

$$
\begin{aligned}
& T_{D}=\left(S_{L}+S_{R}\right) K_{D} \\
& T_{P}=\left(S_{L}+S_{R}\right) K_{P} \\
& T_{I}=\left(S_{L}-S_{R}\right) K_{I} \\
& T_{E}=\left(S_{L}-S_{R}\right) K_{E}
\end{aligned}
$$

where $T_{P}$ is the torque in plantarflexion, $T_{D}$ is the torque in dorsiflexion, $T_{I}$ is the torque in inversion, and $T_{E}$ is the torque in eversion.

Note that the prosthesis' controller always picks either equation 1 or 2 based on the sign of the sum $S_{L}+S_{R}$, and between equations 3 and 4 based on both the sign of the sum $\mathrm{S}_{\mathrm{L}}+\mathrm{S}_{\mathrm{R}}$ and on the sign of the subtraction $\mathrm{S}_{\mathrm{L}}-\mathrm{S}_{\mathrm{R}}$. This is necessary as it is impossible for the foot to generate torques in both dorsiflexion and plantarflexion or in inversion and eversion, simultaneously. Due to the complex shape of the Össur Flex-foot, which is not symmetrical in any axis, the values of the gains $K_{P}, K_{D}, K_{I}$, and $\mathrm{K}_{\mathrm{E}}$ are all different. Since both DP and IE torques are measurable at any point in time, admittance or impedance controllers in both DP and IE can be used at any time during the gait. Impedance controllers similar to those previously presented (32) were successfully implemented in this prosthesis in both DP and IE based on the ground reaction torques described by equations 1-4.

\subsection{IMPEDANCE CONTROLLER}

\subsubsection{IMPEDANCE CONTROLLER DEVELOPMENT}

Similar to the position controllers, two impedance controllers are required to control the interaction of the prosthesis with the environment (Fig. 11.6). The impedance controllers use angle feedback from encoders on each motor (multiplied 
by the gear ratio to estimate the actual foot rotations with respect to the pylon) and the torque feedback from the strain gauges. The controller reference input angle is the sum of DP and IE angles for the left motor controller and the difference of DP and IE angles for the right motor controller. The input reference angles and the angle feedback from the encoders are used to calculate the angular error. The angular error is the input of a PD controller which calculates the desired torque to be generated by the prosthesis. Similar to the angles, the controllers torque feedbacks are the sum of DP and IE torques for the left motor controller, and the difference of DP and IE torques for the right motor controller. The difference between the desired torque and the torque feedback is used in a proportional controller $(\mathrm{P})$ to generate the appropriate motor input. Since the proportional controller affects both the DP and the IE impedances, each torque feedback has its own gain $\left(\mathrm{K}_{\mathrm{DP}}\right.$ and $\mathrm{K}_{\mathrm{IE}}$ for $\mathrm{DP}$ and IE, respectively) and is used to adjust the impedance in each axis independently. The gains $\mathrm{K}_{\mathrm{DP}}$ and $\mathrm{K}_{\mathrm{IE}}$ are identical in the left and right motor controllers.

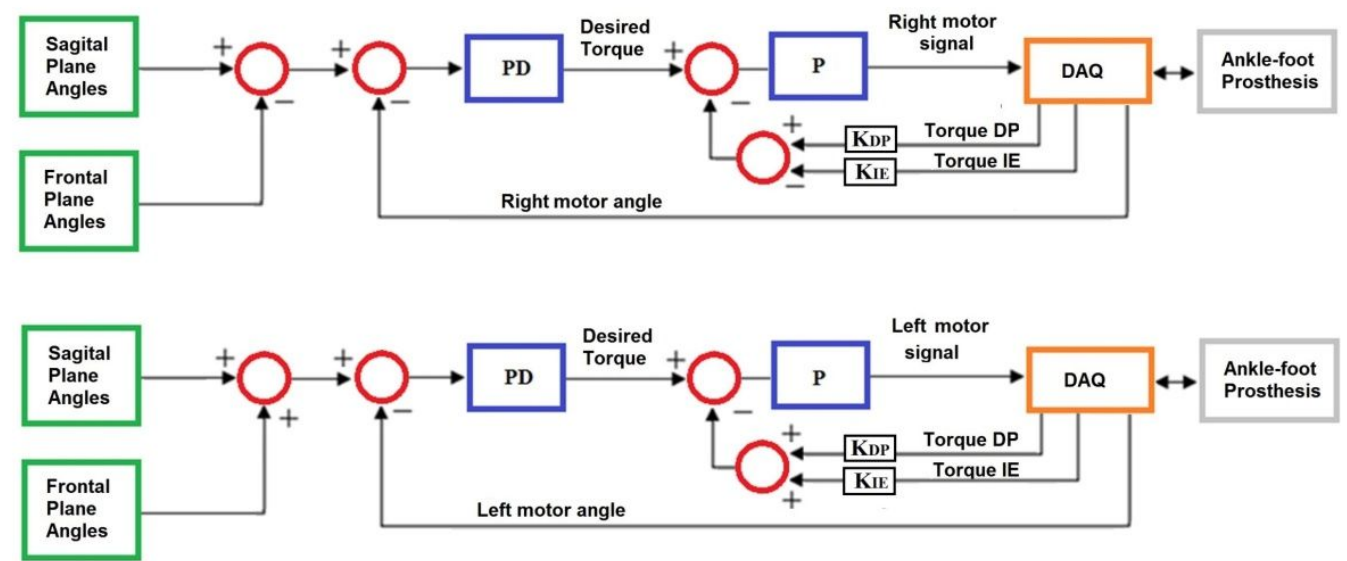

Figure 11.6. Impedance controllers for the left and right motors. The input angle of the right motor is the sum of DP and IE angles while the input angle of the left motor is the difference between the DP and IE angles. The feedback torque of the right motor is the sum of DP and IE torques, while the feedback torque of the left motor is the difference between the DP and IE torques. 


\subsubsection{EXPERIMENTAL ESTIMATION OF THE ANKLE-FOOT PROSTHESIS QUASI-STATIC IMPEDANCE AND IMPEDANCE GAIN CALIBRATION}

An experiment was performed to evaluate the capability of the impedance controller to modulate the quasi-static impedance of the prosthesis in both DP and IE. The experiment was also used to find the relationship between the torque feedback gains $\mathrm{K}_{\mathrm{DP}}$ and $\mathrm{K}_{\mathrm{IE}}$ and the mechanical impedance of the prosthesis. The prosthesis was attached to a rehabilitation robot (Anklebot), as seen in Fig. 11.7. The Anklebot was chosen as it is capable of applying angular disturbances to the prosthesis in both DP and IE while recording the evoked torques. The Anklebot is composed of two linear actuators. If the actuators move in the same direction, the result is a rotation of the prosthesis' foot in DP and if the actuators move in opposite directions, the result is a rotation of the prosthesis' foot in IE. During the tests, the Anklebot mechanically interact with the prosthesis; therefore, it behaves as an impedance component inducing motion in the prosthesis that behaves as an admittance component.

During the tests, the prosthesis was set at a reference angular input of $0^{\circ}$ for both DP and IE. Two independent set of experiments were performed; one to tests the prosthesis in DP, and one to test the prosthesis in IE. The prosthesis was tested with torque feedback gain $\mathrm{K}_{\mathrm{DP}}$ from -0.06 to 0.4 for the DP test and gain $\mathrm{K}_{\mathrm{IE}}$ from -0.1 to 0.4 for the IE test. Small negative feedback gains effectively make the prosthesis stiffer than the passive prosthesis (the prosthesis is passive when the torque feedback gains are set to 0 and the reference angle is also 0 ). However, a large negative torque feedback makes the foot move in the opposite direction as the external force, meaning the foot will move against the external force. During the tests a large negative torque feedback caused the Anklebot to back-drive. Since the Anklebot was back-driving, it was an admittance system instead of an impedance system. While the Anklebot applied a positive force, it was subject to a negative displacement. Since the stiffness calculation follows Hooke's law, it resulted in 
negative stiffness measurements. This is possible as the prosthesis is capable of much larger torques than the Anklebot, which is limited to $23 \mathrm{Nm}$ in DP and $15 \mathrm{Nm}$ in IE. The negative gains were limited to avoid this phenomenon at values of -0.06 for $\mathrm{K}_{\mathrm{DP}}$ and -0.1 for $\mathrm{K}_{\mathrm{IE}}$.

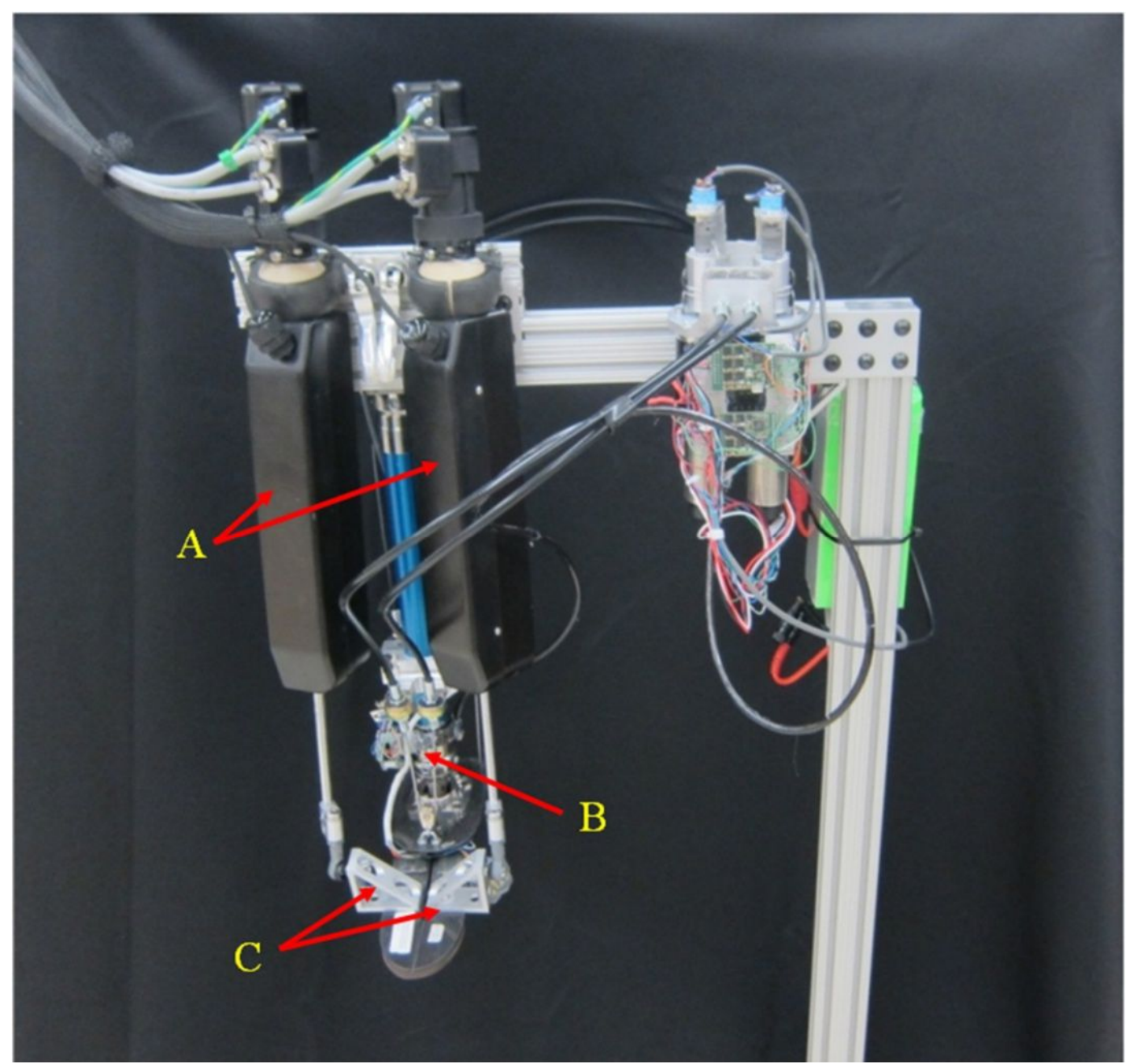

Figure 11.7: Ankle-foot prosthesis attached to the Anklebot for quasi-static impedance estimation. A: Anklebot actuators. B: Ankle-foot prosthesis. C: Connection between the Anklebot and the ankle-foot prosthesis.

The Anklebot was operated in a position control mode with a stiffness of 2177 $\mathrm{Nm} / \mathrm{rad}$ and damping of $100 \mathrm{Nms} / \mathrm{rad}$. These values were found experimentally to generate smooth operation. The Anklebot was tested multiple times with different 
torque feedback gains. In each test, the Anklebot attempted to rotate the prosthesis to 0.4 radians (from the central position) in the positive direction followed by a 0.4 radians rotation in the negative direction and back to the central position with a constant speed of $0.2 \mathrm{rad} / \mathrm{s}$. This cycle was repeated 5 times without any pause. Since the Anklebot torque is limited to $23 \mathrm{~N}-\mathrm{m}$ in DP and $15 \mathrm{~N}-\mathrm{m}$ in IE, the actual range of motion was smaller in the tests where the prosthesis stiffness resulted in forces larger than the Anklebot could generate. The Anklebot recorded the force $\mathrm{F}_{\mathrm{L}}$ and $F_{R}$ for the force in the left and right actuators respectively and the position $X_{L}$ and $\mathrm{X}_{\mathrm{R}}$ for the displacement in the left and right actuators respectively. The data was sampled at a rate of 500 samples per second. The results were filtered with a 0.5 $\mathrm{Hz}$ cutoff frequency to remove sensor noise. The torques and angles in IE direction ( $\tau_{I E}$ and $\theta_{I E}$, respectively) were calculated based on the kinematics model of the experiment as described in equations 5 and 6 . In these equations, D is the distance between the ankle's center of rotation and the actuators end effector in the frontal plane, as shown in Fig. 11.8. Similarly, the torques and angles in DP direction $\left(\tau_{D P}\right.$ and $\theta_{D P}$, respectively) were calculated as described in equations 7 and 8 . In these equations, $\mathrm{L}$ is the distance between the ankle's center of rotation and the actuators end effector in the sagittal plane, as shown in Fig. 11.8.

$$
\begin{gathered}
\tau_{I E}=\frac{\left(F_{R}-F_{L}\right) \times D}{2} \\
\theta_{I E}=\arctan \left(\frac{X_{L}-X_{R}}{D}\right) \\
\tau_{D P}=\left(F_{R}+F_{L}\right) \times L \\
\theta_{D P}=\arctan \left(\frac{X_{L}-X_{R}}{2 L}\right)
\end{gathered}
$$




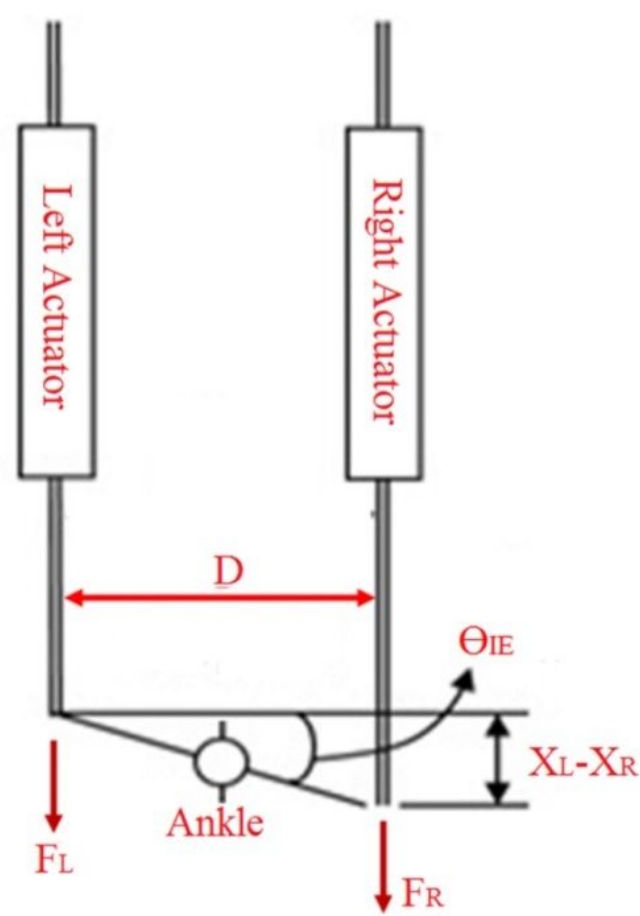

Frontal Plane

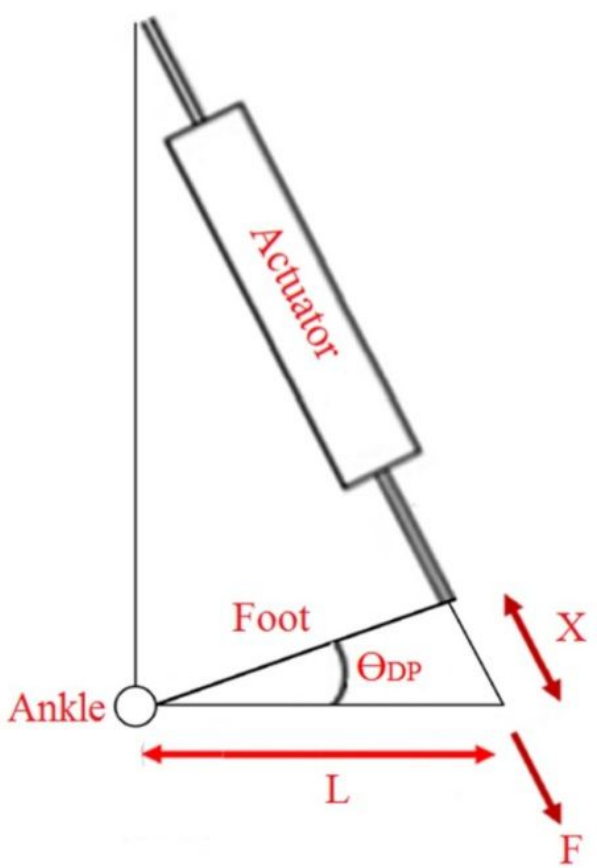

Sagittal Plane

Figure 11.8: Schematics of the Anklebot during the quasi-static stiffness experiments.

The Anklebot rotated the prosthesis in the positive direction followed by a rotation in the negative direction 5 times. This resulted in 5 segments of the prosthesis rotation from positive to negative rotations at each torque feedback gain. Each set of 5 segments were averaged to represent each torque feedback gain. The plots of the averaged torque versus angle at different torque feedback gains are shown in Figs. 11.9 and 11.10 for DP and IE, respectively. The data was plotted within the range of \pm 0.18 rad and was near linear in this range for both DP and IE. The slope of each line is the stiffness of the ankle in $\mathrm{Nm} / \mathrm{rad}$ at that specific torque feedback gain. To estimate the stiffness of the ankle, a second order polynomial was fit to each line on Figs. 11.9 and 11.10 in a least square sense, from where the slopes were obtained. The plot of the estimated stiffness at each torque feedback gain is shown in Fig. 11.11 for DP and Fig. 11.12 for IE. 


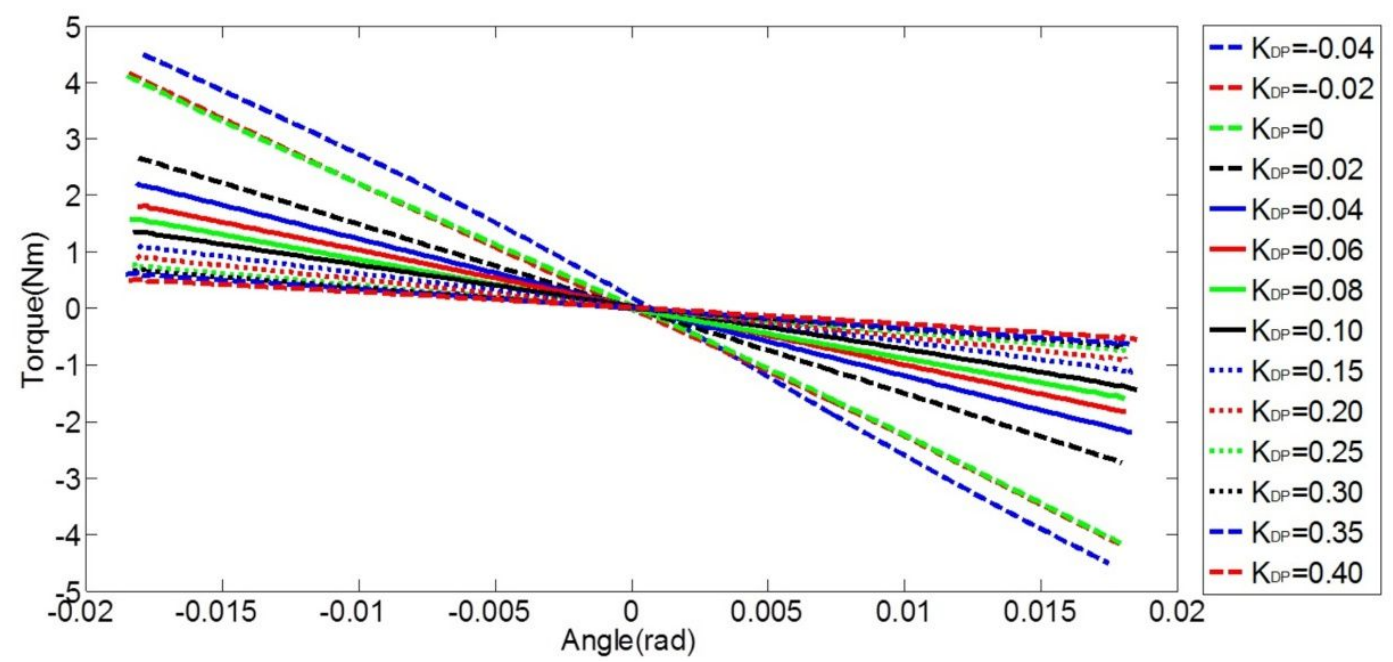

Figure 11.9: Plot of the torque vs. ankle angle of the prosthesis at different torque feedback gains resulted from the quasi-static stiffness tests in DP.

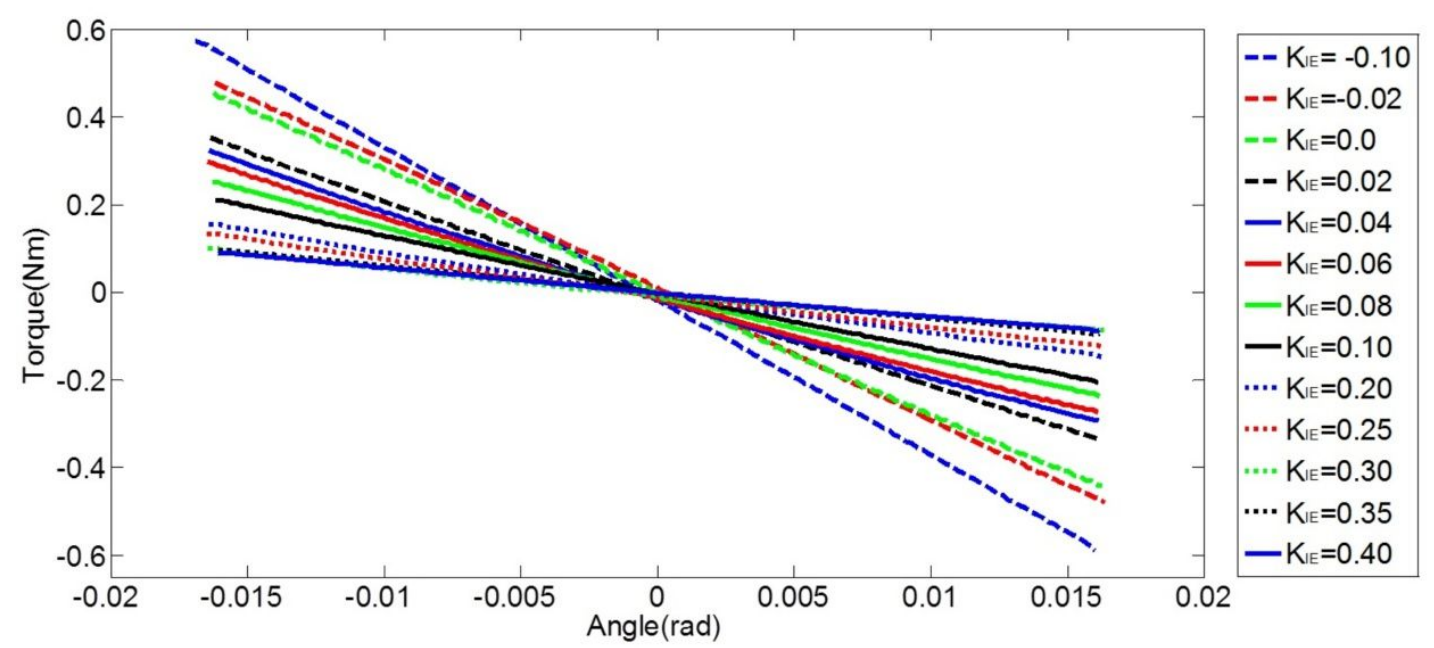

Figure 11.10: Plot of the torque vs. ankle angle of the prosthesis at different torque feedback gains resulted from the quasi-static stiffness tests in IE.

Note that the plots in Figs. 11.9 and 11.10, where both $\mathrm{K}_{\mathrm{DP}}$ and $\mathrm{K}_{\mathrm{IE}}$ were zero, the torque-angle curves were similar to the tests with -0.02 for the torque feedback gains in both axes. The prosthesis did not move when the torque feedbacks were zero, and the stiffness was larger than expected due to static friction in the Bowden cable, gear boxes, and motors. During walking, however, the motors would always 
be moving, even with zero torque feedback, since the reference angles are always changing to generate motion. Due to this characteristic of the zero gain torque feedbacks with constant reference angle, the zero gain torque feedbacks stiffness were not included in the stiffness plots on Figs. 11.11 and 11.12. The stiffness results at zero torque feedback with moving reference angle may be obtained with better precision by interpolating other results near the zero torque feedback.

The stiffness in DP was modulated in the range of $282 \mathrm{Nm} / \mathrm{rad}$ to $22.5 \mathrm{Nm} / \mathrm{rad}$. In IE, the range was between $36 \mathrm{Nm} / \mathrm{rad}$ and $6 \mathrm{Nm} / \mathrm{rad}$. The main goals of the experiments were to validate the capability of the system to modulate the impedance of the prosthesis in both DP and IE and find a correlation between the torque feedback gains and the quasi-static stiffness of the ankle. The correlation between torque feedback gain and stiffness will be used in the control of the prosthesis where time-varying impedance would be needed. A function can be derived from fitting a polynomial to the data on Figs. 11.11 and 11.12. This function would correlate the torque feedback gain to the stiffness. During walk, the prosthesis can obtain a specific stiffness of the ankle by using this equation to calculate the appropriate torque feedback gain which will generate that desired stiffness.

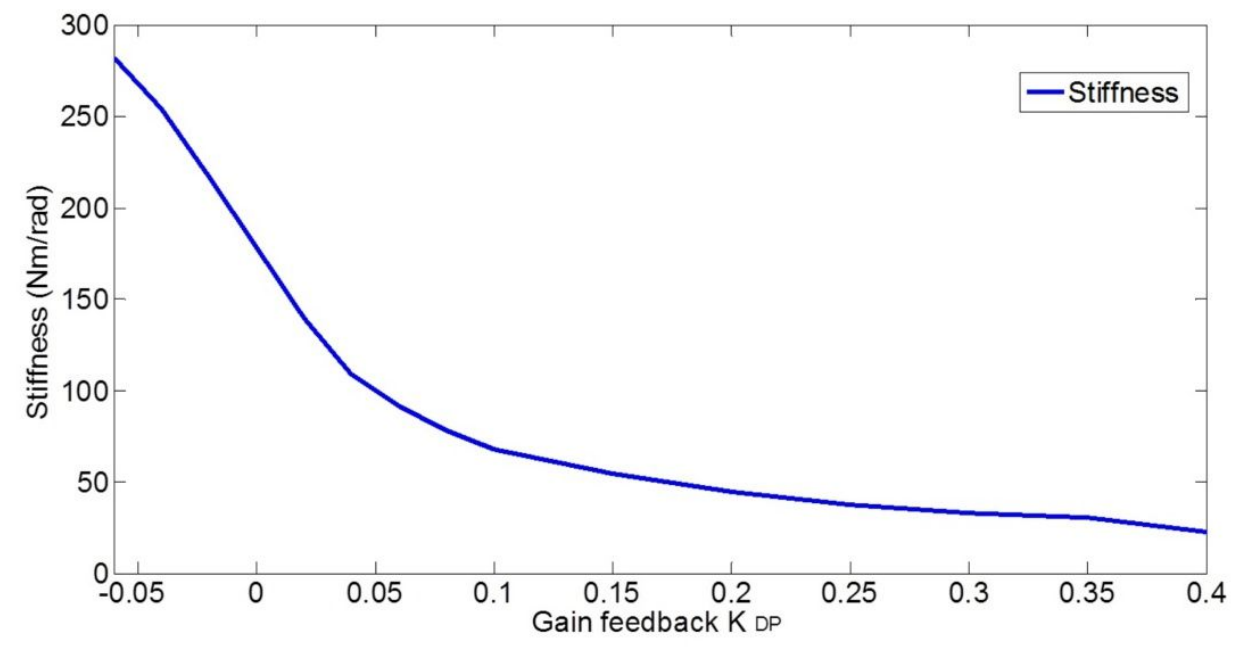

Figure 11.11: Plot of the stiffness vs. torque feedback gain $K_{D P}$ resulted from the quasi-static stiffness tests in DP. 


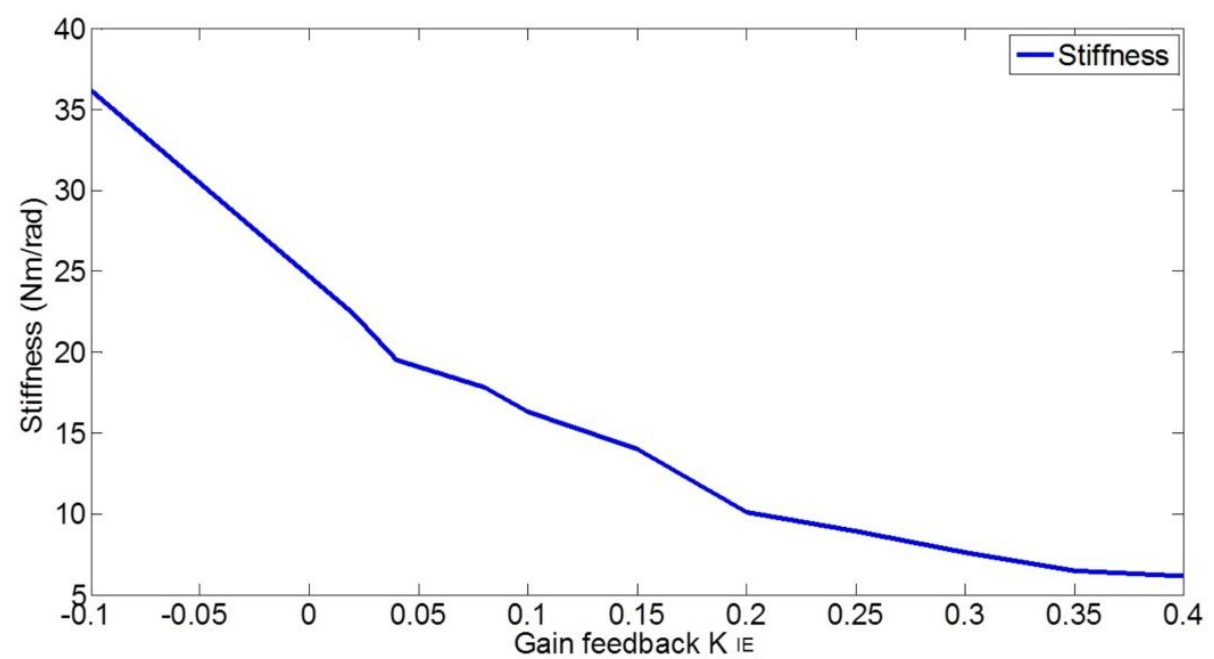

Figure 11.12: Plot of the stiffness vs. torque feedback gain $\mathrm{K}_{\mathrm{IE}}$ resulted from the quasi-static stiffness tests in IE.

\subsection{DISCUSSION}

The presented ankle robot was designed to match the mechanical characteristics of the human ankle including power, range of motion, and weight. The cable-driven design, besides the ability to control the ankle in two DOF, provides significant flexibility in managing the inertia of the prosthesis and allowing amputees with different residual limbs to use the device. Research on optimal place for the actuation system is ongoing. Another advantage of this design feature is the lowprofile ankle-foot mechanism; therefore, the prosthesis can be tailored to fit a wide range of residual limbs.

The recorded ankle rotations in the frontal and sagittal planes were reproduced successfully in the mechanism using a position controller. The mechanism was designed to have larger torques and passive stiffness in the sagittal plane than in the frontal plane, resulting in larger sensitivity to noise and external disturbances in the frontal plane compared to the sagittal plane. The prosthesis was successfully equipped with strain gauges for ground reaction torques measurements to be used on impedance and admittance controllers. 
Impedance controllers were implemented in the prosthesis. Bench tests were performed to estimate the capability of the impedance controllers to modulate the quasi-static impedance of the prosthesis in both DP and IE and to map the torque feedback gains to the changes in the prosthesis' stiffness. The quasi-static impedance test using Anklebot showed that the impedance controller in the prosthesis was capable of modulating its stiffness. In DP, the stiffness ranged from $282 \mathrm{Nm} / \mathrm{rad}$ to $22.5 \mathrm{Nm} / \mathrm{rad}$, showing a ratio of 12.5 from the highest to the lowest stiffness. It has been reported that the stiffness of the human ankle during the stance phase varies from $1.0 \mathrm{Nm} / \mathrm{rad} / \mathrm{kg}$ at the $100 \mathrm{~ms}$ after the heel strike up to 4.6 $\mathrm{Nm} / \mathrm{rad} / \mathrm{kg}$ at $475 \mathrm{~ms}$ after the heel strike in DP (48). This shows a ratio of 4.6 from the highest to the lowest stiffness in the human ankle, showing that the modulation of stiffness in the prosthesis is more than suitable for the human gait. In IE, the range of stiffness was $36 \mathrm{Nm} / \mathrm{rad}$ to $6 \mathrm{Nm} / \mathrm{rad}$. Large stiffness can be obtained by using a stiffer prosthetic foot, stiffer carbon-fiber plate, and by improving the controllers for better stability with higher negative feedback gains. There is no current information (to the best of the author's knowledge) about the stiffness requirements of the foot in IE during walk. Further work is required for proper estimation of the time-varying human ankle impedance in both IE and DP during stance phase of gait to determine if hardware and/or software modifications would be required to modify the stiffness of the prosthesis in either axes.

\subsection{CONCLUSIONS}

This paper describes an ankle-foot prosthesis controllable in the sagittal and frontal planes. Active frontal plane may increase agility by improving turning steps. The prosthesis was designed with similar mechanical characteristics as the human ankle including power, range of motion, and weight. The prosthesis is powered using a cable drive system, allowing for optimal placement of the motors and gear boxes for improving the metabolic efficiency of amputees, and allowing flexibility on the customization of the device to amputees with different residual limb sizes. 
The prosthesis is equipped with impedance controllers in both sagittal and frontal planes. Bench testing was performed to verify the capability of the prosthesis to track the kinematics of the human ankle in two DOF, the ability to modulate the impedance of the ankle, and to map the gains of the impedance controllers to the actual stiffness of the ankle. The prosthesis in the current configuration is capable of properly mimicking the human motion and changing the impedance of the ankle in two DOF in real time with a range of stiffness sufficient for normal human walking. 


\section{2- CONCLUDING REMARKS}

The presented work has shown multiple milestones on the development of a 2DOF robotic ankle-foot prosthesis with anthropomorphic characteristics. The prosthesis has active frontal and sagittal plane control, intended to increase mobility by improving turning steps. This technology potentially will result in increased comfort, decreased overuse or misuse of the individual healthy joints, an increase in activity levels, improvement on obesity and cardiovascular diseases, and overall improving the quality of life of amputees.

The presented work includes the results of the experiments on human subjects for estimations of the human ankle kinetic and kinematics during different types of walking, including straight walking and turning. The development of a robotic ankle-foot prosthesis prototype powered in the frontal and sagittal planes, and the control of the prosthesis using impedance and admittance controllers were presented. Additionally, a 2-DOF perturbation platform for estimation of the timevarying impedance of the human ankle in the frontal and sagittal planes while walking in arbitrary directions were presented. Moreover, the second generation of the 2-DOF robotic prosthesis with a Bowden cable for the power transmission was introduced. The prosthesis was capable of properly mimicking the mechanical characteristics of the human ankle, including power, torque, range of motion, and weight.

The current controller in the prosthesis may benefit by mimicking the actual time-varying impedance of the human ankle through their control strategy. A proper impedance control dynamically maps the time history of ankle angles onto the appropriated time history of ankle torques. Consequently, a possible control strategy is to adjust the torque feedback gain of the impedance controller in real time based on pre-recorded look-up data of the ankle impedance trough the stride cycle. Also, different gait scenarios can be studied such as walking uphill, downhill, climbing steps, turning corners, etc, so the ankle angles and ankle impedance data can be 
estimated. The data from these different gait scenarios will also be evaluated at different gait speeds. In the prosthesis, a more complex finite state machine will be developed to switch between different gait scenarios and the appropriate lookup data of the human ankle impedance and kinematics. For the finite state machine feedback, other than the strain gauges, an inertial measurement unit may be used to enhance the controller's efficiency in conjunction with a vision system to scan the approaching ground profile and anticipate the changes in the gait profile such as stairs and slopes. Future work also will require extensive human testing while amputees use the prosthesis and the prosthesis will be tailored to the specific needs of each amputee.

During the development of the presented work many obstacles had to be overcome. The project started with the assumption that a 2-DOF ankle mechanism could improve steering on amputees, but since this prosthesis is the first of a kind, the research had to be started from scratch. The development of the cable driven mechanism remain in essence the same as the first mockup; however, it has drastically improved by using the Bowden cables for power transmission. The Bowden cables allow for a modular design. For example the motors, gear boxes, and battery, can be incorporated with a quick connector, allowing the removal of these components from the prosthesis in such way that what is left is a passive prosthesis. This can be useful for activities that may not require a powered prosthesis such as walking from one room to another, allowing the user to benefit from either a passive or active prosthesis at his/her choosing. Also, this allows the advances in passive prostheses to be integrated with the advances in controls and mechatronics. The user may easily upgrade or replace components without the need of a whole new prosthesis, reducing cost and giving more options for customization to each individual needs. 


\section{3- REFERENCES}

1. Glaister BC, Bernatz GC, Klute GK, Orendurff MS. Video task analysis of turning during activities of daily living. Gait Posture. 2007;25(2):289-94.

2. Ziegler-Graham K, MacKenzie EJ, Ephraim PL, Travison TG, Brookmeyer R. Estimating the prevalence of limb loss in the United States: 2005 to 2050. Arch Phys Med Rehabil. 2008;89(3):422-9.

3. Robbins J, Strauss G, Aron D, Long J, Kuba J, Kaplan Y. Mortality Rates and Diabetic Foot Ulcers. Journal of the American Podiatric Medical Association. 2008 (6):489-93.

4. NLLIC. Amputation Statistics by Cause Limb Loss in the United States. National Limb Loss Information Center; 2008.

5. Ficanha EM, Rastgaar M, Kaufman KR. Ankle mechanics during sidestep cutting implicates need for 2-degrees of freedom powered ankle-foot prostheses. JRRD. 2015;52:97-112.

6. Orendurff MS, Segal AD, Berge JS, Flick KC, Spanier D, Klute GK. The kinematics and kinetics of turning: limb asymmetries associated with walking a circular path. Gait \& Posture. 2006;23(1):106-11.

7. Ventura JD, Segal AD, Klute GK, Neptune RR. Compensatory mechanisms of transtibial amputees during circular turning. Gait \& Posture. 2011;34:307-12.

8. Bionic Technology with Powered Plantar Flexion. 2012 [cited 2012]; Available from: http://www.iwalkpro.com/Prosthetists.html.

9. The technology behind the PROPRIO FOOT ${ }^{\circledR}$ from Össur. 2012 [cited 2012]; Available from: http://www.ossur.com/?PageID=15736.

10. Endolite, élan. 2012 [cited 2012]; Available from: http://www.endolite.com/products/elan. 
11. Olson NM, Klute GK. Design of a Transtibial Prosthesis With Active Transverse Plane Control. J Med Devices. 2015;9(4).

12. Lamoureux LW, Radcliffe CW. Functional Analysis of the UC-BL Shank Axial Rotation Device. Prosthetics and Orthotics International. 1977;1(2):114-8.

13. Wheeless CR. Wheeless' Textbook of Orthopaedics: Duke University Medical Center's Division of Orthopaedic Surgery; 2012.

14. 2013. Merriam-Webster.

15. A Roadmap for US Robotics, From Internet to Robotics: Computing Community Consortium. 2013; Available from: http:/www.usrobotics.us/reports/CCC\%20Report.pdf.

16. Segal AD, Orendurff MS, Czerniecki JM, Schoen J, Klute GK. Comparison of transtibial amputee and non-amputee biomechanics during a common turning task. Gait Posture. 2011 Jan;33(1):41-7.

17. Hase K, Stein RB. Turning Strategies During Human Walking. J Neurophysiol. 1999;81(6):2914-22.

18. Glaister BC, Orendurff MS, Schoen JA, Bernatz GC, Klute GK. Ground reaction forces and impulses during a transient turning maneuver. J Biomechanics. 2008;41(4):3090-3.

19. Ficanha EM, Rastgaar M, Moridian B, Mahmoudian N, editors. Ankle Angles during Step Turn and Straight Walk: Implications for the Design of a Steerable Ankle-Foot Prosthetic Robot Dynamic Systems and Controls Conference 2013; Stanford University, Palo Alto, CA.

20. Palmer M. Sagittal plane characterization of normal human ankle function across a range of walking gait speeds. Cambridge, MA: Massachusetts Institute of Technology; 2002. 
21. Gates DH. Characterizing ankle function during stair ascent, descent, and level walking for ankle prosthesis and orthosis design. Boston, MA: Boston University; 2004.

22. Davis R, DeLuca P. Gait characterization via dynamic joint stiffness. Gait and Posture. 1996;4(3):224-31.

23. Collins SH, Kuo AD. Recycling Energy to Restore Impaired Ankle Function during Human Walking. PLoS ONE. 2010;5(2).

24. Hansen AH, Childress DS, Miff SC, Gard SA, Mesplay KP. The human ankle during walking: implications for design of biomimetic ankle prostheses. Journal of Biomechanics. 2004;37:1467-74.

25. Goldfarb M. Powered Robotic Legs - Leaping Toward the Future. National Institute of Biomediacl Imaging and Bioengineering. 2010.

26. Sup F, Bohara A, Goldfarb M. Design and Control of a Powered Transfemoral Prosthesis. The International Journal of Robotics Research. 2008;27:263-73.

27. Sup F, Varol HA, Mitchell J, Withrow TJ, Goldfarb M. Preliminary Evaluations of a Self-Contained Anthropomorphic Transfemoral Prosthesis. IEEE ASME Trans Mechatron. 2009;14(6).

28. Sup F. A Powered Self-Contained Knee and Ankle Prosthesis For Near Normal Gait in Transfemoral Amputees. Nashville, Tennessee: Vanderbilt University; 2009.

29. Au S. Powered Ankle-Foot Prosthesis for the Improvement of Amputee Walking Economy. Cambridge, MA: Massachusetts Institute of Technology; 2007.

30. Au S, Herr H. Powered ankle-foot prosthesis. Robotics \& Automation Magazin 2008;15(3):52 - 9. 
31. Au S, Weber J, Herr H. Powered Ankle-foot Prosthesis Improves Walking Metabolic Economy. IEEE Transactions on Robotics. 2009;25(1):51-66.

32. Au S, Herr H, Weber J, Martinez-Villalpando EC, editors. Powered AnkleFoot Prosthesis for the Improvement of Amputee Ambulation. International Conference of the IEEE, Engineering in Medicine and Biology Society; 2007; Lyon.

33. Herr HM, Grabowski AM, editors. Powered Ankle-Foot Prosthesis Improves Metabolic Demand of Unilateral Transtibial Amputees During Walking. Amer Society of Biomechanics; 2010; Long Beach, CA.

34. Herr HM, Grabowski AM. Bionic ankle-foot prosthesis normalizes walking gait for persons with leg amputation. Proc Biol Sci. 2012 Feb 7;279(1728):457-64.

35. Taylor MJD, Dabnichki P, Strike SC. A three-dimensional biomechanical comparison between turning strategies during the stance phase of walking. Human Movement Science. 2005;24:558-73.

36. Strike SC, Taylor MJ. The temporal-spatial and ground reaction impulses of turning gait: is turning symmetrical? Gait Posture. 2009;29(4):597-602.

37. Sasaki K, Neptune RR. Differences in muscle function during walking and running at the same speed. Journal of Biomechanics. 2005;39.

38. Sheppard JM, Young WB. Agility literature review: classifications, training and testing. J Sports Sciences. 2006;24(9):919-32.

39. Colborne GR, Naumann S, Longmuir PE, Berbrayer D. Analysis of mechanical and metabolic factors in the gait of congenital below knee amputees. American Journal of Physical Medicine \& Rehabilitation. 1992;92:272 - 8.

40. Molen NH. Energy/speed relation of below-knee amputees walking on motor-driven treadmill. Internationale Zeitschrift für angewandte Physiologie einschließlich Arbeitsphysiologie. 1973;31(3):173-85. 
41. Winter DA, Sienko SE. Biomechanics of below-knee amputee gait. Journal of Biomechanics. 1988;21(5):361-7.

42. Skinner HB, Effeney DJ. Gait analysis in amputees. American Journal of Physical Medicine \& Rehabilitation. 1985;64:82-9.

43. Bateni H, Olney S. Kinematic and kinetic variations of below-knee amputee gait. Journal of Prosthetics \& Orthotics. 2002;14(1):2- 13.

44. Adamczyk PG, Kuo AD, editors. Asymmetry in Amputee Gait: The Propagating Effects of Weak Push-Off. American Society of Biomechanics; 2011; Long Beach, CA.

45. Ferris AE, Aldridge JE, Sturdy JT, Wilken JM, editors. Evaluation of the Biomimetic Properties of a New Powered Ankle-Foot Prosthetic System. American Society of Biomechanics; 2011; Long Beach, CA.

46. Arndt A, Wolf P, Liu A, Nester C, Stacoff A, Jones R, et al. Intrinsic foot kinematics measured in vivo during the stance phase of slow running. Journal of Biomechanics. 2007;40:2672-8.

47. Shamaei K, Sawicki GS, Dollar AM. Estimation of Quasi-Stiffness and Propulsive Work of the Human Ankle in the Stance Phase of Walking. PLoS ONE. 2013;8(3):e59935.

48. Rouse EJ, Hargrove L, Perreault E, Kuiken T. Estimation of Human Ankle Impedance During Walking Using the Perturberator Robot. Fourth IEEE RAS/EMBS International Conference on Biomedical Robotics and Biomechatronics; Roma, Italy2012.

49. Rouse EJ, Hargrove L, Perreault E, Peshkin M, Kuiken T. Development of a Mechatronic Platform and Validation of Methods for Estimating Ankle Stiffness during the Stance Phase of Walking. Journal of biomechanical engineering. 2013;135(8):10091-8. 
50. Rastgaar M, Ho P, Lee H, Krebs HI, Hogan N, editors. Stochastic estimation of multi-variable human ankle mechanical impedance. ASME Dynamic Systems and Control Conference; 2009; Hollywood, CA.

51. Rastgaar M, Ho P, Lee H, Krebs HI, Hogan N, editors. Stochastic estimation of the multi-variable mechanical impedance of the human ankle with active muscles. ASME Dynamic Systems and Control Conference; 2010; Boston, MA.

52. Lee H, Ho P, Krebs HI, Hogan N, editors. The multi-variable torquedisplacement relation at the ankle. ASME Dynamic Systems and Control Conference; 2009; Hollywood, CA.

53. Lee H, Ho P, Rastgaar M, Krebs HI, Hogan N, editors. Quantitative Characterization of Steady-State Ankle Impedance with Muscle Activation. ASME Dynamic Systems and Control Conference 2010; Cambridge, MA.

54. Lee H, Ho P, Rastgaar M, Krebs HI, Hogan N. Multivariable static ankle mechanical impedance with relaxed muscles. Journal of Biomechanics. 2011;44:1901-8.

55. Lee H, Ho P, Rastgaar M, Krebs HI, Hogan N. Multivariable static ankle mechanical impedance with active muscles. IEEE Transaction on Neural Systems and Rehabilitation Engineering. 2014;22(1):44-52.

56. Ho P, Lee H, Krebs HI, Hogan N. Directional Variation of Active and Passive Ankle Static Impedance. ASME Dynamic Systems and Control Conference; Holleywood, CA2009.

57. Ho P, Lee H, Rastgaar M, Krebs HI, Hogan N, editors. The Interpretation of the Directional Properties of Voluntarily Modulated Human Ankle Impedance. ASME Dynamic Systems and Control Conference; 2010; Cambridge, MA.

58. Lee H, Krebs HI, Hogan N, editors. Linear Time-Varying Identification of Ankle Mechanical Impedance During Human Walking. ASME 2012 5th Annual Dynamic Systems and Control Conference; 2012; Fort Lauderdale, FL, USA. 
59. Scott SH, Winter DA. Talocrural and talocalcaneal joint kinematics and kinetics during the stance phase of walking. Journal of Biomechanics 1991;24(8):743-52.

60. Wu G, Siegler S, Allard P, Kirtley C, Whittle M . ISB recommendation on definitions of joint coordinate system of various joints for the reporting of human joint motion--part I: ankle, hip, and spine. International Society of Biomechanics. Journal of Biomechanics. 2002;35:543-8.

61. Health and Wellness for Persons with Disabilities Today. Office of the Surgeon General; 2007.

62. Donelan JM, Kram R, Kuo AD. Mechanical work for step-to-step transitions is a major determinant of the metabolic cost of human walking. Journal of Experimental Biology. 2002;205:3717-27.

63. Donelan JM, Kram R, Kuo AD. Simultaneous positive and negative external work in human walking. Journal of Biomechanics. 2002;35:117-24.

64. Ruina A, Bertram JE, Srinivasan M. A collisional model of the energetic cost of support work qualitatively explains leg sequencing in walking and galloping, pseudoelastic leg behavior in running and the walk-to-run transition. Journal of Theoretical Biology. 2005;237(2):170-92.

65. Kuo AD. Energetics of actively powered locomotion using the simplest walking model. Journal of Biomechanical Engineering. 2002;124:113-20.

66. Kuo AD, Donelan JM, A. Ruina. Energetic consequences of walking like an inverted pendulum: Step-to-step transitions. Exercise and Sport Sciences Reviews. 2005;33:88-97.

67. Klute GK, Czerniecki J, Hannaford B, editors. Development of powered prosthetic lower limb. Proceedings of the 1st National Meeting, Veterans Affairs Rehabilitation Research and Development Service; 1998; Washington, DC. 
68. Hitt J, Merlo J, Johnston J, Holgate M, Boehler A, Hollander K, et al., editors. Bionic running for unilateral transtibial military amputees. 27th Army Science Conference (ASC); 2010; Orlando, Florida.

69. Hitt JK, Sugar TG, Holgate M, Bellman R. An Active Foot-Ankle Prosthesis With Biomechanical Energy Regeneration. J Med Devices. 2010;4(1).

70. Eilenberg MF, Geyer H, Herr H. Control of a Powered Ankle-Foot Prosthesis Based on a Neuromuscular Model. IEEE Transactions on Neural Systems and Rehabilitation Engineering. 2010;18(2):164-73.

71. Grabowski AM, D’Andrea S. Effects of a powered ankle-foot prosthesis on kinetic loading of the unaffected leg during level-ground walking. Journal of NeuroEngineering and Rehabilitation. 2013;10(49).

72. Gailey R, Allen K, Castles J, Kucharik J, Roeder M. Review of secondary physical conditions associated with lower-limb amputation and long-term prosthesis use. Journal of Rehabilitation Research \& Development. 2008;45(1):15-30.

73. Harlaar J, Becher J, Snijders C, Lankhorst G. Passive stiffness characteristics of ankle plantar flexors in hemiplegia. Clinical Biomechanics. 2000;15(4):261-70.

74. Singer B, Dunne J, Singer K, Allison G. Evaluation of triceps surae muscle length and resistance to passive lengthening in patients with acquired brain injury. Clinical Biomechanics 2002;17(2):151-61.

75. Chung SG, Rey E, Bai Z, Roth EJ, Zhang L-Q. Biomechanic changes in passive properties of hemiplegic ankles with spastic hypertonia. Archives of Physical Medicine and Rehabilitation. 2004;85(10):1638-46.

76. Rydahl SJ, Brouwer BJ. Ankle stiffness and tissue compliance in stroke survivors: A validation of myotonometer measurements. Archives of Physical Medicine and Rehabilitation. 2004;85(10):1631-7. 
77. Kobayashi T, Leung AL, Akazawa Y, Tanaka M, Hutchins SW. Quantitative measurements of spastic ankle joint stiffness using a manual device: A preliminary study. Journal of Biomechanics. 2010;43(9):1831-4.

78. Lamontagne A, Malouin F, Richards CL. Viscoelastic behavior of plantar flexor muscle-tendon unit at rest. The Journal of Orthopaedic and Sports Physical Therapy. 1997;26(5):244-52.

79. Hunter IW, Kearney RE. Dynamics of Human Ankle Stiffness: Variation with Mean Ankle Torque. Journal of Biomechanics. 1982;15(10):742-52.

80. Kearney RE, Hunter IW. Dynamics of human ankle stiffness: Variation with displacement amplitude. Journal of Biomechanics. 1982;15(10):753-6.

81. Kearney RE, Hunter IW. System identification of stretch reflex dynamics. Crit Rev Biomed Eng. 1990;18:55-87.

82. Weiss PL, Kearney RE, Hunter IW. Position dependence of ankle joint dynamics - I. Passive mechanics. Journal of Biomechanics. 1986;19(9):727-35.

83. Weiss PL, Kearney RE, Hunter IW. Position dependence of ankle joint dynamics-II. Active mechanics. Journal of Biomechanics. 1986;19(9):737-51.

84. Kearney RE, Stein RB, Parameswaran L. Identification of intrinsic and reflex contributions to human ankle stiffness dynamics. IEEE transactions on biomedical engineering. 1997;44(6):493 - 504.

85. Kirsch RF, Kearney RE. Identification of time-varying stiffness dynamics of the human ankle joint during an imposed movement. Experimental Brain Research. $1997 ; 114: 71-85$.

86. Mirbagheri MM, Kearney RE, Barbeau H, editors. Quantitative, objective measurement of ankle dynamic stiffness: intra-subject reliability and intersubject variability. 18th Annual International Conference of the IEEE Engineering in Medicine and Biology Society; 1996; Amsterdam. 
87. Sinkjaer T, Toft E, Andreassen S, Hornemann BC. Muscle stiffness in human ankle dorsiflexors: Intrinsic and reflex components. Journal of Neurophysiology. 1998;60(3):1110-21.

88. Roy A, Krebs HI, Williams DJ, Bever CT, Forrester LW, Macko RM, et al. Robot-aided neurorehabilitation: A novel robot for ankle rehabilitation. IEEE Transactions on Robotics and Automation. 2009;25(3):569-82.

89. Saripalli A, Wilson S. Dynamic Ankle Stability and Ankle Orientation. 7th Symp Footwear Biomech Conf; Cleveland, OH2005.

90. Zinder SM, Granata KP, Padua DA, Gansneder BM. Validity and reliability of a new in vivo ankle stiffness measurement device. Journal of Biomechanics. 2007;40:463-7.

91. Fitzpatrick RC, Taylor JL, McCloskey DI. Ankle Stiffness of Standing Humans in Response to Imperceptible Perturbation: Reflex And Task-Dependent Components. Journal of Physiology. 1992;454:533-47.

92. Loram ID, Lakie M. Human balancing of an inverted pendulum: position control by small, ballistic-like, throw and catch movements. The Journal of Physiology. 2002;540(3):1111-24.

93. Loram ID, Lakie M. Direct measurement of human ankle stiffness during quiet standing: the intrinsic mechanical stiffness is insufficient for stability. The Journal of Physiology. 2002;545(3):1041-53.

94. Sasagawa S, Ushiyama J, Masani K, Kouzaki M, Kanehisa H. Balance control under different passive contributions of the ankle extensors: quiet standing on inclined surfaces. Exp Brain Res. 2009;196(4):537-44.

95. Winter DA, Patla AE, Rietdyk S, Ishac MG. Ankle muscle stiffness in the control of balance during quiet standing. Journal of Neurophysiology. 2001;85(6):2630-3 
96. Morasso PG, Sanguineti V. Ankle muscle stiffness alone cannot stabilize balance during quiet standing. Journal of Neurophysiology. 2002;88(4).

97. Farley CT, Blickhan R, Saito J, Taylor CR. Hopping frequency in humans: a test of how springs set stride frequency in bouncing gaits. Journal of Applied Physiology. 1991;71:2127- 32.

98. Farley CT, González O. Leg stiffness and stride frequency in human running. Journal of Biomechanics. 1996;29(2):181-6.

99. Rouse EJ, Hargrove LJ, Peshkin MA, Kuiken TA, editors. Design and validation of a platform robot for determination of ankle impedance during ambulation. Conf Proc IEEE Eng Med Biol Soc; 2011; Boston, Massachusetts, USA.

100. Richter H, Simon D, Smith WA, Samorezov S. Dynamic modeling, parameter estimation and control of a leg prosthesis test robot. Applied Mathematical Modelling. 2014

101. Kang HG, Dingwell JB. Separating the effects of age and walking speed on gait variability. Gait Posture. 2008;27(4):572-27.

102. Hogan N. Impedance Control: An Approach to Manipulation: Part ITheory. J Dyn Sys, Meas, Control 1985;107(1):1-7.

103. Carignan C, Cleary K. Closed-loop force control for haptic simulation of virtual environments. Haptics-e. 2000;1:01-1.

104. Rouse EJ, Hargrove LJ, Perreault EJ, Kuiken TA. Estimation of Human Ankle Impedance During the Stance Phase of Walking. Ieee Transactions On Neural Systems And Rehabilitation Engineering. 2014;22(4):870-8.

105. Winter DA, Patla AE, Rietdyk S, Ishac MG. Ankle Muscle Stiffness in the Control of Balance During Quiet Standing. J Neurophysiol. 2001;85:2630-3. 
106. Lortie M, Kearney RE. Identification of physiological systems: estimation of linear timevarying dynamics with non-white inputs and noisy outputs. Med Biol Eng Comput. 2001;39:381-90.

107. Samuel K. Au JW, and Hugh Herr Biomechanical Design of a Powered Ankle-Foot Prosthesis. In: IEEE, editor. International Conference on Rehabilitation Robotics,; Noordwijk, The Netherlands2007.

108. Rastgaar M, Lee H, Ficanha E, Ho P, Krebs H, Hogan N. Multi-directional Dynamic Mechanical Impedance of the Human Ankle; A Key to Anthropomorphism in Lower Extremity Assistive Robots. In: Artemiadis P, editor. Neuro-Robotics: Springer Netherlands; 2014. p. 157-78.

109. Weyand PG, Sternlight DB, Bellizzi MJ, Wright S. Faster Top Running Speeds are Achieved with Greater Ground Forces Not More Rapid Leg Movements. J Appl Physiol. 2000;89:1991-9.

110. Nester CJ, Findlow AF, BSc PB, Bowden PD. Transverse Plane Motion at the Ankle Joint. Foot \& Ankle International. 2003.

111. Levine D, Richards J, Whittle M. Whittle's Gait Analysis. fifth ed: Evolve; 2012.

112. Lee H, Krebs HI, Hogan N. Multivariable Dynamic Ankle Mechanical Impedance with Active Muscles. IEEE Transactions on Neural Systems and Rehabilitation Engineering. 2014;22(5):971-81.

113. Ficanha E, Rastgaar M. Stochastic estimation of human ankle mechanical impedance in medial-lateral direction. Dynamic Systems and Control Conference San Antonio, TX,USA2014.

114. Ludvig D, Visser TS, Giesbrecht H, Kearney RE. Identification of TimeVarying Intrinsic and Reflex Joint Stiffness. Ieee Transactions on Biomedical Engineering. 2001;58(6):1715-23. 
115. Zhao Y, Westwick DT, Kearney RE. Subspace Methods for Identification of Human Ankle Joint Stiffness. IEEE Transaction of Biomedical Engineering. 2010.

116. Lee H, Hogan N. Time-Varying Ankle Mechanical Impedance during Human Locomotion. IEEE Transactions on Neural Systems and Rehabilitation Engineering. 2014;62(DOI: 10.1109/TNSRE.2014.2346927).

117. Ficanha EM, Rastgaar M, Kaufman KR. Control of a 2-DOF powered ankle-foot mechanism. 2015 IEEE International Conference on Robotics and Automation; 25 May - 30 May 2015; Seattle, WA, USA2015.

118. Ficanha EM, Rastgaar M, Kaufman KR. A two-axis cable-driven anklefoot mechanism. Robotics and Biomimetics. 2014;1(17).

119. Glaister BC, Schoen A, Orendurff MS, Klute GK. A Mechanical Model of the Human Ankle in the Transverse Plane During Straight Walking: Implications for Prosthetic Design. J Biomech Eng. 2009;131(3).

120. Glaister BC, Schoen JA, Orendurff MS, Klute GK. Mechanical behavior of the human ankle in the transverse plane while turning. IEEE Trans Neural Syst Rehabil Eng. 2007;15(4):552-9.

121. Lee H, Patterson T, Ahn J, Klenk D, Lo A, Krebs HI, et al. Static Ankle Impedance in Stroke and Multiple Sclerosis: A Feasibility Study. Annual International Conference of the IEEE EMBS; Boston, Massachusetts2011.

122. Lee H, Krebs HI, Hogan N. Multivariable Dynamic Ankle Mechanical Impedance with Relaxed Muscles. IEEE Transactions on Neural Systems and Rehabilitation Engineering. 2014.

123. Ficanha E, Ribeiro G, Aagaah MR. Instrumented walkway for estimation of the ankle impedance in dorsiflexion-plantarflexion and inversion-eversion during standing and walking. Dinamic Systems and Control Conference; Columbus Ohio 2015. 
124. Browning R, Modica J, Kram R, Goswami A. The effects of adding mass to the legs on the energetics and biomechanics of walking. Medicine and science in sports and exercise. 2007;39(3):515. 


\title{
14- APPENDICES
}

\subsection{APPENDIX A - DOCUMENTATION FOR CHAPTER 4}

9102015

\author{
Aighslink by Copyright Clearance Center
}

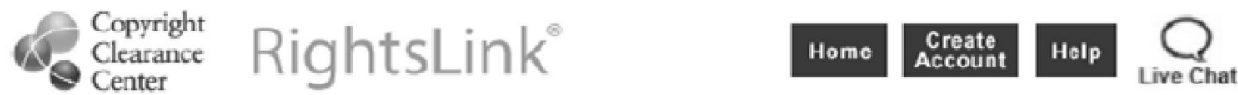

Pe.
pequosting
permission
to reuse
content from
an IEEE
publieation

\author{
Title: Ankle kinematics describing gait \\ ogility: Considerations in the \\ design of an agile ankle-foot \\ prosthesis \\ Conference Biomedical Róbotics and \\ Proceedings: Biomechatronics (2014 5th \\ IEEE RAS \& EMBS International \\ Conterence on \\ Author: Ficanha, E.M.; Ruiyu Kang; \\ Rastgaar, M. \\ Publisher: IFEF \\ Date: 12-15 Aug. 2014 \\ Copyright 92014 , IEEE
}

Thesis / Dissertation Reuse

The IEEE does not require individuals working on a thesis to obtain a formal reuse license, however, you may print out this statement to be used as a permission grant:

Requirements to be followed when using any portion (e.g., figure, graph, table, or textual material) of an IEEE copyrighted paper in a thesis:

1) In the case of textual material (e.g.i using short quotes or referring to the work within these papers) users must give full credit to the original source (author, paper, publication) followed by the IEEE copyright line (c) 2011 IEEE,

2) In the case of illustrations or tabular material, we require that the copyright line (c) [Year of original publication] IEEE appear prominently with each reprinted figure and/or table:

3) If a substantial portion of the original paper is to be used, and if you are not the senior author, also obtain the senior author's approval.

Requirements to be followed when using an entire IEEE copyrighted paper in a thesis:

1) The following IEEE copyright/ credit notice should be placed prominently in the references: (G) [year of original publication] IEEE, Reprinted, with permission, from [author names, paper title, IEEE publication title, and month/year of publication]

2) Only the accepted version of an IEEE copyrighted paper can be used when posting the paper or your thesis on-line.

3) In placing the thesis on the author's university website, please display the following message in a prominent place on the website: In reference to IEEE copyrighted material which is used with permission in this thesis, the IEEE does not endorse any of [university/educational entity's name goes here]"s products or services, Internal or personal use of this material is permitted. If interested in

reprinting/republishing IEEE copyrighted material for advertising or promotional purposes or for creating new collective works for resale or redistribution, please go to

http://wwwieee,ora/publications standards/publications/rights/riahts link, htm to learn how to obtain a License from RightsLink.

If applicable, University Microfilms and/or Proquest Library, or the Archives of Canada may supply single coples of the dissertation.

\section{BACK}

Copyright 2015 Copyright Qlearance Center, InG, All Rights Reserved, Privacy statement, Ierms and Conditions, Comments? We would like to hear from you, E-mail us at customercarealicoonrightucom 


\title{
14.1 APPENDIX B - DOCUMENTATION FOR CHAPTER 5
}

\author{
$09 / 22 / 2015$ \\ Evandro Ficanha \\ 205 W Hougton Ave. \\ Houghton MI, 49931 \\ (906)369-7383
}

Dear Tristan A. Horrom

I am completing a doctoral dissertation at Michigan Technological University entitled "Anthropomorphic powered ankle-foot prosthesis with 2 degrees of freedom based on the mechanical properties of the human ankle" I would like your permission to reprint in my dissertation excerpts from the following: Volume 52 Number 1, 2015 Pages $97-112$ Ankle mechanics during sidestep cutting implicates need for 2-degrees of freedom powered ankle-foot prostheses. The requested permission extends to any future revisions and editions of my dissertation. These rights will in no way restrict republication of the material in any other form by you or by others authorized by you. Your signing of this letter will also confirm that you own [or your company owns] the copyright to the above- described material.

If these arrangements meet with your approval, please acknowledge your agreement and return to me by e-mail

Sincerely,

Evandro M. Ficanha

Horrom, Tristan

$11: 36 \mathrm{AM}(2$ hours ago)

$h$

to $\mathrm{me} \nabla$

Dear Evandro,

Thank you for the notification. Because we are a government publication, all of the work we published is considered in the public domain and is not under copyright. We do ask that proper citation is given for any work from our journal that is reused, but no specific permissions are needed. As long as you acknowledge that this material was originally published in our journal, you are free to use the material in your dissertation.

Please let me know if you have any further questions.

--Tristan

Tristan A. Horrom, MS

Technical Writer/Editor

Veterans Health Administration

Journal of Rehabilitation Research and Development

George H. Fallon FB, $1^{\text {st }}$ Floor, Suite 102

31 Hopkins Plaza

Baltimore, MD 21201

443-759-3437

Fax: 410-962-9670 


\title{
14.1 APPENDIX C- DOCUMENTATION FOR CHAPTER 6 9102015

\author{
Rightslink Printable Lionse
}

SPRINGER LICENSE

TERMS AND CONDITIONS

Sep 10, 2015

This is a License Agreement between evandro ficanha ("You") and Springer ("Springer") provided by Copyright Clearance Center ("CCC"). The license consists of your order details, the terms and conditions provided by Springer, and the payment terms and conditions.

\author{
All payments must be made in full to Ccc, For payment instructions; please see \\ information listed at the bottom of this form. \\ License Number \\ License date \\ Licensed content publisher \\ Licensed content publication \\ Licensed content title \\ Licensed content author \\ Licensed content date \\ Type of Use \\ Portion \\ 3705440584850 \\ Sep 10, 2015 \\ Springer \\ Springer eBook \\ Multi-axis Capability for Powered Ankle-Foot Prostheses \\ Evandro M, Ficanha \\ Jan 1,2014 \\ Thesis/Dissertation \\ Excerpts \\ Author of this Springer article Yes and you are a contributor of the new work \\ Order reference number \\ None \\ Title of your thesis / \\ dissertation \\ ANTHROPOMORPHIC POWERED ANKLE-FOOT PROSTHESIS WITH 2 \\ DEGREES OF FREEDOM BASED ON THE MECHANICAL PROPERTIES \\ OF THE HUMAN ANKLE \\ Expected completion date \\ Dec 2015 \\ Estimated size(pages) \\ 150 \\ Customer Tax ID \\ usMichigan \\ Total \\ 0.00 USD \\ Terms and Conditions \\ Introduction \\ The publisher for this copyrighted material is Springer Science + Business Media. By \\ clicking "accept" in connection with completing this licensing transaction, you agree that the \\ following terms and conditions apply to this transaction (along with the Billing and Payment \\ terms and conditions established by Copyright Clearance Center, Inc. (" $\mathrm{CCC}$ ), at the time \\ that you opened your Rightslink account and that are available at any time at \\ http://mraccount.copvrighticom).
}

Limited License

With reference to your request to reprint in your thesis material on which Springer Science and Business Media control the copyright, permission is granted, free of charge, for the use indicated in your enquiry. 
9/102015

Rightslink Printable License

Licenses are for one-time use only with a maximum distribution equal to the number that you identified in the licensing process.

This License includes use in an electronic form, provided its password protected or on the university's intranet or repository, including UMI (according to the definition at the Sherpa website: http://www.sherpa.ac.uk/romeo/). For any other electronic use, please contact Springer at (permissions.dordrecht@springer.com or permissions.heidelberg@springer.com).

The material can only be used for the purpose of defending your thesis limited to universityuse only. If the thesis is going to be published, permission needs to be re-obtained (selecting "book/textbook" as the type of use).

Although Springer holds copyright to the material and is entitled to negotiate on rights, this license is only valid, subject to a courtesy information to the author (address is given with the article/chapter) and provided it concerns original material which does not carry references to other sources (if material in question appears with credit to another source, authorization from that source is required as well).

Permission free of charge on this occasion does not prejudice any rights we might have to charge for reproduction of our copyrighted material in the future.

Altering/Modifying Material: Not Permitted

You may not alter or modify the material in any manner. Abbreviations, additions, deletions and/or any other alterations shall be made only with prior written authorization of the author(s) and/or Springer Science + Business Media. (Please contact Springer at (permissions.dordrecht@springer.com or permissions.heidelberg@springer.com)

Reservation of Rights

Springer Science + Business Media reserves all rights not specifically granted in the combination of (i) the license details provided by you and accepted in the course of this licensing transaction, (ii) these terms and conditions and (iii) CCC's Billing and Payment terms and conditions.

Copyright Notice:Disclaimer

You must include the following copyright and permission notice in connection with any reproduction of the licensed material: "Springer and the original publisher/journal title, volume, year of publication, page, chapter/article title, name(s) of author(s), figure number(s), original copyright notice) is given to the publication in which the material was originally published, by adding; with kind permission from Springer Science and Business Media"

Warranties: None

Example 1: Springer Science + Business Media makes no representations or warranties with respect to the licensed material.

Example 2: Springer Science + Business Media makes no representations or warranties with respect to the licensed material and adopts on its own behalf the limitations and disclaimers established by CCC on its behalf in its Billing and Payment terms and conditions for this licensing transaction. 
\$102015

Righisink Prirublo Licanse

Indemnity

You hereby indemnify and agree to hold harmless Springer Science + Business Media and $\overline{C C C}$, and their respective officers, directors, employees and agents, from and against any and all claims arising out of your use of the licensed material other than as specifically authorized pursuant to this license.

No Transfer of License

This license is personal to you and may not be sublicensed, assigned, or transferred by you to any other person without Springer Science + Business Media's written permission.

No Amendment Except in Writing

This license may not be amended except in a writing signed by both parties (or, in the case of Springer Science + Business Media, by CCC on Springer Science + Business Media's behalf).

Objection to Contrary Terms

Springer Science + Business Media hereby objects to any terms contained in any purchase order, acknowledgment, check endorsement or other writing prepared by you, which terms are inconsistent with these terms and conditions or CCC's Billing and Payment terms and conditions. These terms and conditions, together with CCC's Billing and Payment terms and conditions (which are ineorporated herein), comprise the entire agreement between you and Springer Science + Business Media (and CCC) conceming this licensing transaction. In the event of any conflict between your obligations established by these terms and conditions and those established by CCC's Billing and Payment terms and conditions, these terms and conditions shall control.

Jurisdiction

All disputes that may arise in connection with this present License, or the breach thereof, shall be settled exclusively by arbitration, to be held in The Netherlands, in accordance with Dutch law, and to be conducted under the Rules of the 'Netherlands Arbitrage Instituut' (Netherlands Institute of Arbitration). $O R$ :

All disputes that may arise in connection with this present License, or the breach thereof, shall be settled exclusively by arbitration, to be held in the Federal Republic of Germany, in accordance with German law.

Other terms and conditions:

$\mathbf{v 1 . 3}$

Questions? customercaredecopvriahticom or +1-855-239-3415 (toll free in the US) or $+1=978-646=2777$. 


\subsection{APPENDIX D- DOCUMENTATION FOR CHAPTERS 7}

\section{AND 8}

Beth Darchi <DarchiB@asme.org>

to me $\nabla$

Dear Mr. Ficanha:

This letter has been revised to reflect all requests. It is our pleasure to also grant you permission to use all or any part of the following ASME papers:

- "Impedance and Admittance Controller for a Multi-Axis Powered Ankle-Foot Prosthesis," by Evandro M. Ficanha and Mohammad Rastgaar, Paper No. DSCC2014-6032,

- "Gait Emulator for Evaluation of Ankle-Foot Prostheses Capable of Turning 1," by Evandro Ficanha; Mohammad Rastgaar; Kenton R. Kaufman, J. Med. Devices. 2015; 9(3)

cited in your letter for inclusion in a doctoral dissertation entitled Anthropomorphic powered ankle-foot prosthesis with 2 degrees of freedom based on the mechanical properties of the human ankle to be published by Michigan Technological University.

Permission is granted for the specific use as stated herein and does not permit further use of the materials without proper authorization. Proper attribution must be made to the author(s) of the materials. Please note: if any or all of the figures and/or Tables are of another source, permission should be granted from that outside source or include the reference of the original source. ASME does not grant permission for outside source material that may be referenced in the ASME works.

As is customary, we request that you ensure full acknowledgment of this material, the author(s), source and ASME as original publisher. Acknowledgment must be retained on all pages printed and distributed.

Many thanks for your interest in ASME publications.

Sincerely,

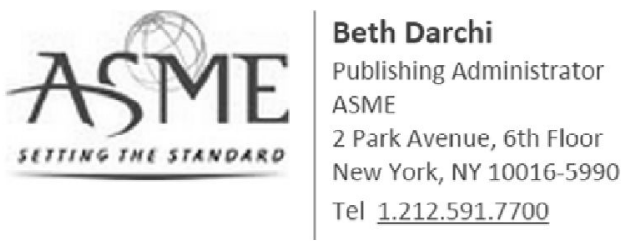

